\title{
ZMLA 00620
}

\section{Leniclopteron'um Gatalouls}

editus $a b$

Embrik Strand

Pars 54:

H. Stichel

Amathusiidae

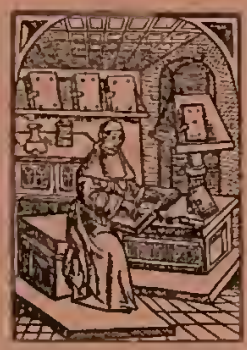

W. Junk

Berlin W. 15

17. II. 1933. 


\section{Lepidopterorum Catalogus}

Pars 1: Ch. Aurivillius, Chrysopolomidae. 1911. 4 p.

2: A. Pagenstecher, Callidulidae. 1911. 14 p.

3: A. Pagenstecher, Libytheidae. 1911. $12 \mathrm{p}$.

4: H. Wagner et R. Pfitzner, Hepialidae. 1911. 26 p.

5: E. Strand, Noetuidae: Agaristinae. 1912. 82 p.

6: E. Meyriek, Adclidne, Micropterygidae, Graeilariadae. 1912. 68 p.

7: II. Zerny, Syntomidae. 1912. 179 p.

8: L. B. Prout, Geometridae: Brephinae, Oenoehrominae. 1912. 94 p.

9: P. Mabille, Hesperidae: Subf. Pyrrhopyginae. - McDunnough, Megathymidae. 1912. $22 \mathrm{p}$.

10: E. Ileyrick, Tortrieidae. 1912. 86 p.

11: H. Eltringham et K. Jordan, Nymphalidae: Subfam. Aeraeinae. 1913. $65 \mathrm{p}$.

12, 18, 21, 23: H. Wagner, Sphingidae. 1913-1919. 420 p.

13: E. Meyrick, Carposinidae, Heliodinidae, Glyphipterygidae. 1913. $53 \mathrm{p}$.

14: I. B. Prout, Geometridae: Subfam. Hemitheinae. 1913. 192 p.

15: K. W. ron Dalla Torre, Castniidae: Subfam. Castniinae, Neocastniinae, Pemphigostolinae. 1913. 28 p.

16: E. Strand, Brahmaeidae. - H. G. Dyar et E. Strand: Megaloprgidae, Dalceridae, Epipyropidae. 1913. 35 p.

17: E. Meyrick, Pterophoridae, Orneodidae. 1913. 44 p.

19: E. Meyrick, Hyponomeutidae, Plutellidae, Amphitheridae. 1914. $64 \mathrm{p}$.

20: K. W. ron Dalla 'lorre, Thyrididae. 1914. 55 p.

22, 24, 26, 45: E. Strand et F. Bryk, Arctiidae. 1919-1931. 957 p.

25: K. W. rou Dalla Torre, Cymatophoridae. 1921. 38 p.

27: F. Bryk, Baroniidae, Teinopalpidae, Parnassiidae. 1923. 247 p.

28: K. W. von Dalla Tor'e et E. Straud, Lepidarbelidae - E. Strand, Heterogynididae. 1923.14 p.

29: K. W. rou Dalla Torve, Cossidae. 1923. 63 p.

30: K. W. von Dalla Torre, Epiplemidae, Uraniidae. 1924. 57 p.

31: K. W. von Dalla Torre et E. Strand, Aegeriidae. 1925. 282 p.

32: R. van Eceke, Cochlidionidae (Limacodidae). 1925. 81 p.

33: H. Burgeff, Zjgaenidae I: Generis Zygaeng palnearetica pars. 1926. $91 \mathrm{p}$.

34: K. W. von Dalla Torre et E. Strand, Psyehidac. 1929. 215 p.

Fortsetzung siehe 3 . Umsehlagseite. 
Der "Lepidopterorum Catalogus" ist nunmehr im Erscheinen soweit fortgeschritten, $\mathrm{da} B$ er - treten keine unvorhergesehenen Hindernisse ein - in etwa drei Jahren abgeschlossen sein dürfte, und daß jetzt die Einteilung des Werkes in Bände erfolgen kann. Die Titel-Blätter für diese Band-Einteilung werden, soweit die dazugehörigen Familien schon vollständig erschienen sind, zusammen.mit einer Gesamt-Übersicht einer der nächsten Versendungen beigefügt. Auch werden schon jetzt Bestellungen auf Einband-Decken entgegengenommen.

\section{Der Verlag.}


as sy 


\title{
Lepidopterporum Catalogus
}

editus $a b$

\author{
Embrik Strand
}

Pars 54:

H. Stichel

Amathusiidae

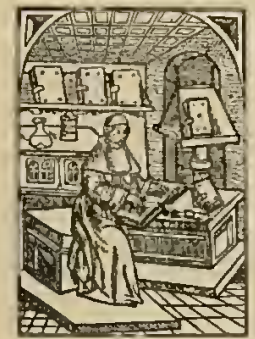

W. Junk

Berlin W. 15

1933.

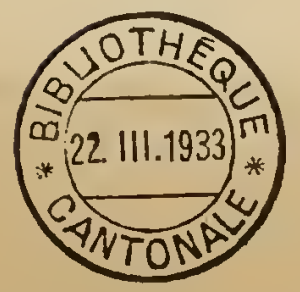




\section{Familia Amathusiidae ${ }^{*}$ )}

Auct. H. Stichel

Amathusiinae (Subfam.), F. Moore, Lep. Ind., v. 2, p. 170 (1893). - Nicéville, Journ. Bombay Nat. Hist. Soc., v. 9, p. 259 (1894), - Nicéville \& L. Martin, Journ. Asiat. Soc. Bengal, v. 64. II, p. 392 (1896). - L. Martin, Deutsch. Ent. Zeit. Lop., v. 8, 1895, p. 253 (1896). - Amathusiidi (Tribus) (part.), E. Reuter, Acta Soc. Sci. Fenn., v. 22, p. 108 (1896). - Amathusiinae, Crowley, Ann. Mag. Nat. Hist., ser. 6, v. 17, p. 66 (1896). - A. (part.), J. Davidson, Bell \& Aitken, Journ. Bombay Nat. Hist. Soc., v. 10, p. 245 (1896). - E. Y. Watson, 1. c., v. 10, p. 652 (1897). - Amathusinae, Fruhstorfer, Berlin. Ent. Zeit., v. 41, 1896, p. 301; v. 42, p. 4 (1897). - Amathusiinae, Nicéville, Journ. Bombay Nat. Hist. Soc., v. 12, p. 187 (1898). - Nicéville \& Elwes, Journ. Asiat. Soc. Bengal, v. 66. II, 1897 , p. 681 (1898). - Nicéville \& Manders, 1. c. v. 68. II, p. 186 (1899). - "Amathusien", Fruhstorfer, Deutsch. Ent. Zeit. Lep., v. 12, p. 71 (1899). - Amathusiinae, Nicéville, Joum. Asiat. Soc. Bengal, v. 69. II, p. 220 (1900). - Crowley, Proc. Zool. Soc. London, p. 505 (1900). - Nicéville, Journ. Bombay Nat. Hist. Soc., v. 13, p. 165 (1900). - Id., Journ. Asiat. Soc. Bengal, v. 71. II, p. 8 (1902). - Amathusiidae + Discophoridae, H. Stichel. Deutsch. Ent. Zeit., v. 15, p. 59 (1902). - Amathusiinae, Shelford, Journ. Straits Branch Asiat. 'Soc., Nr. 41, p. 106 (1904). - A. + Discophorinae + Hyantinae, H. Stichel, Gen. Ins., fasc. 36, 31, 39 (1906 \& 05). - Amathusiidae, Fruhstorfer, Ent. Zeit. Guben, v. 20 , p. 146 (1906). - Amathrsiinae, id., Soc. Entom., 22, p. 106 (1907). - "Amathusiiden", id., Deutsch. Tnt. Zeit. Iris, v. 24, p. 54 (1910). - Amathusiidae, id.; A. Seitz, Großschmett. Erde. v. 9, p. 403 (1911). - J. C. Moulton, Ent. Monthly Mrag., v. 48, p. 81 (1912). Id., Entomol., v. 45, p. 246 (1912). - Amathusiidae, H. Stichel, Tiorreich, Lief. 34, p. 1 (1912). - Strand, Arch. Naturg., v. 78, 1912, Abt. A; Heft 11, p. 65, 77 (1913); ill., 1. c. v. 79, 1913, Abt. A, Heft 7, p. 183. - Amathusinae, R. Sonth, Journ. Bombay Nat. Hist. Soc., v. 22, p. 352 (1913). - Amathusiinae, Moulton, Journ. Straits Branch Asiat. Soc., Nr. 65, p. 13, 17 (1913). - G. A. Waterhouse \& Lyell, Butterfl. Austral., p. 15, 48 (1914). - Amathusiidae, Strand, Int. Ent. Zeit., v. 8, p. 104 (1914). - Amathusiinae. W. Rothschild, Nov. Zool., v. 22, p. 124, 201 (1915). - Amathusiidae, Röber, Nova Guinea, v. 13, Zool., v. 1, p. 47 (1915). - Tecke, l. c., p. 66 (1915). - Joicey, Noakes \& Talbot, Trans. Ent Soc. London, p. 366 (1915). - Amathusidae, Amathusinae, W. Rothschild (\& Durrant), Rep. Brit. Ornithol. Exp. Dutch N. Guinea, 2 Nr. 15, p. 17, 18 (1915). - Amathusiidae, W. Rothschild, Nov. Zool., v. 22, p. 124, 201 (1915). - Strand, Lep. Niepeltiana, v. 2, p. 11 (1916). - Fruhstorfer, Arch. Naturg., v. 81, 1915, Abt. A, Heft 11, p. 64 (1916). - Joicey \& Talbot, Ann. Mag. Nat. Hist., sor. 8, v. 17, p. 73 (1916). - Amathusiinae, W. Rothschild, Nov. Zool., v. 23, p. 299 (1916). - Amathusinae, Godfrey, Journ. Nat.

*) Die erreichbare Literatur ist bis einschließlich 1930 berücksichtigt. 
Hist. Soc. Siam, v. 2, p. 123 (1916). - Amachusiidae (laps.!), Fruhstorfer, Ent. Rundschau, 34, p. 44 (1917). - Amathusiidae, Strand, Soc. Entom., v. 33, p. 12 (1918). - Eecke, Zool. Mededeel. Leiden. v. 4, p. 83 (1918). - Amathusiinae, Rebel, Verh. Zool.-Botan. Ges. Wien, v. 68, p. (53) (1918). - Amathusiidae (Amathusiinae + Discophorinae), W. Rothschild, Journ. Feder. Malay. Stat. Mus., v. 8, p. 153 (1920). - Amathrsiidae, Joicey \& Talbot, Bull. Hill Mus., v. 1, p. 172 (1921). - W. H. Evans, Journ. Bombay Nat. Hist. Soc., p. 793 (part.) (1923). - Eecke, Nova Guinea, v. 15, Zool., p. 37 (1924). - Amathusidae, Strand, Bull. Soc. Zool. France, v. 51, p. 397 (1926). - Amathusiinae, liebel, Verh. Zool.-Botan. Ges. Wien, v. 74-75, p. (201) (1926). - Godfrey, Jonrn. Siam Soc. Nat. Hist., Suppl., v. 7, p. 122,262 (1927). - Amathusiidae (part.), W. H. Evans, Ident. Ind. Butterfl., p. 24, 84 (1927). - A., A. Seitz, Ent. Rundschau, v. 45, p. 24 (1928). - Joicoy \& Talbot, Bull. Hill Mas., v. 2, p. 184 (1928). - A. F. Rosa, Entomologist, v. 62 , p. 226 (1929). - Eocke, Treubia, v. 7, p. 359 (1929). - Amathusiinae, Godfrey, Journ. Siam Soc. Nat. Hist. Suppl., v. 7, p. 257 (1930).

He li con $i$ i (part.), Linné, Syst. Nat., ed. 10, p. 465 (1758). - H. (part.), p. $43+$ Nymphales (part.), p. 77, Turton, Gen. Syst. Nat. Linné, v. 3. II (1806).

Equites Achivi (part.), p. $186+$ Heliconii (part.), p. 231, Linné-Houttuyn, Natuuri. Hist., v. 1. XI (1766-69). E. A. (part.) p. $748+H$. (part.), p. 754, Linné, Syst. Nat., ed. 12 (1767). - E. A. (part.), p. $571+H$. (part.), p. 581, P. L. S. Müller, Naturs. Linné, v. 5. I (1774). Equites (part.), p. 10 + Danai (part.) + Nymphales (p.). p. 11, P. Cramer, Pap. Exot., v. 1 (1775). - E. Achivi (part.), p. $449+$ Heliconii (part.), p. 459, J. C. Fabricius, Syst. Ent. (1775). - E. A. (part.), p. $46+H$. (part.), p. $89+$ Danai Festivi (part.), p. $186+$ Nymphales Gemmati (part.), p. 227, Goeze, Entom. Beytr., ఛ. 3. I (1779). - Nymphalis G. (part.), C. Stoll, Essai Syst. Lep.: P. Cramer, Pap. Exot., v. 4, App., p. 7 (1782). - E. Achiui (part.), p. $10+H$. (part.), p. $27+$ Danai festiui (part.), p. $52+N$. G. (part.), p. 68, J. O. Fabricius, Spec. Ins., v. 2 (1781) - E. Archivi (!) (part.), p. 6 + Heliconii (part.), p. $13+D . f$. (part.), p. $25+N . g$. (part.), p 34, id., Mant. Ins., v. 2 (1787). - E. Achivi, Esper, Ausl. Schmett., t. 56 (1788). - E. Achiui (part.), v. 3, p. $5+$ Heliconii (part.), v. 4, p. $84+$ Consules (part.), v. 6. p. $5+$ Praetores (part.), v. 8, p. 7 (Jablonsky \&) Herbst, Naturs. Ins., Schmett. (1788-96). - E. A. (part.), p. $2234+H$. (part.), p. $2249+$ Danai festivi (part.), p. $2273+$ Nymphales Qemmati (part.), p. 2290, Gmelin-Linné, Syst. Nat., ed. 13, v. 1. V (1790). - E. A. (part.), p. 21 + Festivi (part.), p. 39 + Nymphales (part.), p. $61+$ Heliconii (part.), p. 159, J. C. Fabricius, Ent. syst., v. 3. I (1793). - Nymphales (part.), Donovan, Epitome Ins. Ind., Index, p. 2 (1800). - Festivi (part.), p. 1, Danai F. (part.), p. 2, id., Epitome Ins. China, Index (1798).

Oreades (Dubiae) (part.), v. 1, Index p. 2 + Potamides (Superbae) (part.) $\neq$ Oreades (Distinctae) (part.), จ. 2, Index, p. 2, Jac. Hübner, Samml. Exot. Schmett., (180626). - Potamides (Stirps) (part.), p. 46: Conspicuae (Fam.) (part.), p. 50, Moerae (Coitus) (part.), p. 51 + Oreades (Stirps) (part.), p. 52: Dubiae (Fam.) (part.), p. 52, Taenares (Coitus) (part.), p. 52, id. Verz. Schmett. (1818). 
Papilionides (part.), Latreille, Gen. Crust. Ins., v. 4, p. 194 (1809). - Papilionidae (part.), F. Moore: J. Anderson, Anat. Zool. Results Exp. Yunnan, v. 1, p. 923 (1878).

Hipparchidae, Hipparchiae (part.), Swainson, Zool. Illustr., Text ad t. 2; Add. (1820).

Perlata (Sectio) (part.), Latreille, Fam. Règne Anim., p. 468 (1825). - A. Berthold: Latreille, Nat. Fam., p. 473 (1827).

$T h y s a n u r i f o r m$ - Stirps (part.), Horsfield, Descr. Cat. Lep. E.-Ind. Comp., p. 61 (1828). - Thysanuriform Larvae (Stirps with) (part.), Horsfield \& F. Moore, Cat. Lep. Mus. E.-Ind. Comp., v. 1, p. 184 (1857).

Satyridac (part.), Swainson \& Shuckard, Hist. Nat. Arrang. Ins. (Cab. Cyel. Lardner, v. 129), p. 93 (1840). C. \& R. Felder, Reise Novara, v. 2. II, p. 453 (1866). Hewitson, Exot. Butt., v. 3 (4), Text ad t. [35] Zouxidia \& Aemona; Index (1868). - "Satyriden", I. Martin, Mitt. Münch. Entom. Ges., v. 1, p. 71 (1910). - Satyridae (part.), N. D. Riley, Zool. Record, v. 58, 1921, pars 11, p. 172 (1923). - N. D. Riley \& Gabriel, Cat. Type Spec. Rhop. Brit. Mus., pars 1, p. 5 (1924). - Imperial Bureau Entom., Zool. Record, v. 59, 1922, pars 11, p. 302 et vol. sequent. (1924 etc.).

Satyromorpha (part.), E. Haase, Deutsch. Ent. Zeit. Lep., v. 4 p. 33 (1891) (conf. A. Seitz, Ent. Zeit. Stettin, v. 53, p. 157 (1892). - Satyromorphes (!) (part.), Ie Cerf, Enc. Ent., ser. B. 3, Lep., v. 1, p. 141 (1926). Satyromorphi (!) (part.), A. Seitz (sec. E. Haase), Ent. Rundschau, v. 45, p. 3 (1928).

Nymphalidac (part.), J. O. Westwood: Donovan, Epitome Ins. China, n. ed., p. 62 (1842). - N. (part.) p. 64 + Morphidae (part.) j. 115, E. Doubleday, List Lep. Brit. Mus., pars 1 (1844). - N. (part.), id., 1. c., App., p. 25, 29 (1848). - N. (part.), E. Doubleday (\& Westwood), Gen. Diurn. Iep., v. 1, p. 81 (1847). - J. O. Westwood, Cab. Orient. Ent., p. 9 etc. (1848). - Nymphalitae (part.), p. 110 + Morphitae (part.) (Divis.) (part.), p. 164, Chenu \& H. Lucas, Enc. Hist. Nat., Papill. (1851). - Nymphalidae (part.), p. 21 + Morphidae (part.), p. 37, Ménétriés, Enum. Corp. $\Lambda$ nim. Ae. Petropol., Lep., pars 1 (1855). - N. (part.), p. $230+M$. (part.), p. 232, Carus-Gerstäcker, Handb. Zool., v. 2 (1863). - Nymphalina (part.), Herrich-Schäffer, Corr.-Bl. Zool.-Min. Ver. Regensb., v. 18, p. 95, 105; v. 19, p. 74 (1864\& 65). - Nymphalinae (part.), H. W. Batee, Journ. Entom., v. 2, p. 164, 177 (1864). - Nymphalidae (part.), F. Moore, Proc. Zool. Soc. London, p. 761 (1865). - Hewitson, Exot. Butt., v. 3 (4), t. [53] Zeuxidia \& Aemona (1865). - A. G. Butler, Trans. Ent. Soc. London, p, $485(1870)$. - W. F. Kirby, Cat. Diurn. Lep., p. 1 atque autor. numer. systemat. congruent. (1871 et anni sequent.) - A. G. Butler, Proc. Zool. Soc. London, p. 765 (1876). - Nymphaline (part.), Piepers (\& P. C. T. Snellen), Tijdschr. Ent., v. 19, p. 146 (1876). - Nymphalidae (part.), p. 466 + Morphinae (part.), p. 468, A. G. Butler, Proc. Zool. Soc. London (1877). - Nymphalinae (part.), F. Moore, 1. c., p. 826 (1878). - Id., Lep. Oeylon, v. 1. I, p. 26 (1880). - N. (part.), p. $302+$ Morphinae (part.), p. 300, W. F. Kirby, Scient. Proc. R. Dublin Soc., ser. 2, v. 2 (1880). - Nymphalidae (part.), Ch. 
Oberthür, Ann. Mus. Stor. Nat. Genova, v. 15, p. 496 (1880). - Nymphalinae (part.), p. 66, 423 + Morphina (Group), p. 67, 428, Distant, Rhop. Malay. (1882 \& 86). N. (part.), p. $24+$ Morphinae (part.), p. 20, Kheil, Rhop. Nias (1884). - "Nymphaliden" (part.), p. $86+$,Morphiden" (part.), p. 86, 187, O. Staudinger (\& Schatz), Exot. Schmett., v. 1 (1885 \& 86). - Nymphalinae (part.), Nicéville, Journ. Asiat. Soc. Bengal, v. 54. II, p. 2, 43 (1885). - Nymphalidae (part.), J. O. Westwood, Trans. Ent. Soc. London, p. 467 (1888). - Nymphalina (part.), P. C. T. Snellen, Tijdschr. Entom., v. 32, p. 388 (1889). - Nymphalidae (part.), Skertchly, Ann. Mag. Nat. Hist., ser. 6, v. 3, p. 210 (1889). - C. O. Waterhouse, Aid Ident. Ins., v. 2, Index, p. 15 (1890). - „Nymphaliden" (part.), E. Hofmann, Jahresh. Ver. Naturk. Württemb. v. 46, p. 236 (1890). - Nymphalidae (part.), E. Y. Watson, Journ. Bombay Nat. Hist. Soc., v. 5, p. 29 (1890). - N. (part.), p. $341+$ Morphinae, p. 344, Nicéville, l. c., v. 6 (1891). $N$. (part.), p. $435+M .$, p. 437, H. S. Fergusson, 1. c., v. 6 (1891). - N. (part.), P. C. 'T. Snellen, Midden Sumatra, v. 4. VIII, p. 16 (1892). - N. (part.), p. $251+$ Morphinae (part:), p. 257, Tryon: J. P. Thomson, Brit. N. Guin., App. 3 (1892). - Nymphalides (part.), Robbe, Ann. Soc. Ent. Belgique, v. 36, p. 128 (1892). - Nymphalidae (part.) + Marphiinne (part.), Chapman, Ent. Record Journ. Var., v. 11, p. 87 (1899). - N. (part.), L. C. H. Young, Journ. Bombay Nat. Hist. Soc., v. 15, 1903, p. 295, 269, 483 (1903 \& 04). - H. Stichel, Gen. Ins., fasc. 31, 36, 39 (1905 \& 06). - Moulton, Journ. Straits Branch Asiat. Soc., Nr. 63, p. 78 (1912). - Imperial Bureau of Entom., Zool. Record, v. 59, pars 11, p. 297 (1924). - Antram, Butterfl. Ind,, p. 68 (1924).

Morphides (part.), Boisduval, Spec. Gén. Lép., v. 1, p. 166 (1836). - Morphites (part.), E. Blanchard, Hist. An. Artic., v. 3, p. 454 (1840). - Id., Hist. Ins., v. 2, p. 334 (1845). - Morphidae (part.), (E. Doubleday \&) Westwood, Gen. Diurn. Lep., v. 2, p. 332 (1851). - J. O. Westwood, Trans. Ent. Soc. Londou, n. ser., v. 4, p. 163, 169 (1856). - Hewitson, Exot. Butt., v. 4 (3), t. [1,2] Clerome, Drusilla \& Hyantis; Index (1862). - Morpho (Fam.) (part.), Weidemeyer, Proc. Ent. Soc. Philad., v. 2, sep. p. 25 (1864). - Morphinae (part.), A. G. Butler, Cat. Diurn. Lrep. Fabr., p. 42 (1869). - Id., Lep. Exot., p. 28 (1870). $M$., id., Trans. Ent. Soc. London, p. 485 (1870). - $M$. (part.s), p. 115 + Nymphalinae (part.), p. 147, W. F. Kirby, Cat. Diurn. Lep. (1871). - Morphinae (part.), Herb. Druce, Proc. Zool. Soc. London, p. 340 (1873). - Id., l. c., p. 104 (1874). - A. G. Butler, 1. c., p. 279 (1874). - J. G. Wood, Ins. abroad, p. 391 (1874). - A. G. Butler, Trans. Linn. Soc. London, n. ser., v. 1, p. 538 (1876). Morphidae (part.), Wallace, Geogr. Distr. Anim., v. 1, p. 319, 364, 404 (1876). - "Morphinen" (part.), F, Müller, Jena. Zeit. Naturw., v. 11, p. 101 (1877). - Morphinae, W. F. Kirby, Cat. Diurn. Lep., Suppl., p. 714, App., p. 846 (1877). - Id., Entomologist, v. 10, p. 290 (1877). - $M$. (part.), O. Salvin \& F. D. Godman, Proc. Zool. Soc. London, p. 143 (1877). - A. G. Butler, l. c., p. 468 (1877). - F. D. Gadman \& O. Salvin, 1. c., p. 638,645 (1878). F. Moore, 1. c., p. 826 (1878). - Morphidae, Wood-Mason, 
Journ. Asiat. Soc. Bengal, v. 47. II, p. 175 (1878). Morphinae (part.), A. G. Butler, Trans. Linn. Soc. London, ser. 2 Zool., v. 1, p. 538 (1879). - W. F. Kirby, Cat. Coll. Diurn. Lep. Hewitson, p. 106 (1879) (vide O. Orüger, Berlin. Ent. Zeit., v. 25, p. 111, 1881). - Wood-Mason \& Nicéville, Journ. Asiat. Soc. Bengal, v. 50. II, p. 245 (1881). - M., A. G. Butler, Proc. Zool. Soc. London, p. 605 (1881). - Nicéville, Journ. Asiat. Soc. Bengal, v. 51. II, p. 57 (1882). - Id., l. c., v. 52. II, p. 93 (1883), G. F. L. Marshall \& Nicéville, Butt. Ind. Burm. Ceyl., v. 1. II, p. 281, 282 (1883). - ,Morphiden", B. Gerhard, Berlin. Berlin. Ent. Zeit., v. 27, p. 181 (1883). - Morphinae, A. G. Butler, Ann. Mag. Nat. Hist., ser. 5, v. 11, p. 411 (1883). - A. Pagenstecher, Jahrb. Nassau. Ver. Naturk., v. 37, p. 178 (1884). - Nicéville, Journ. Asiat. Soc. Bengal, v. 54. II, p. 43 (1885). - A. G. Butler, Ann. Mag. Nat. Hist., ser. 5, v. 16, p. 302 (1885). - $M$. (part.). G. W. Müller, Zool. Jahrb. System., v. 1, p. 607, 616 (184, 193) (1886). - F. Moore, Journ. Linn. Soc. London, v. 21, p. 33 (1886). - Morphidae (part.), Doherty, Journ. Asiat. Soc. Bengal, v. 55. II, p. 109, 121 (1886). - Ribbe, Corr.Bl. Ver. Iris, v. 1, p. 82 (1886). - Morphides (part.), Capronnier, Ann. Soc. Ent. Belgique, v. 30, p.6 (1886). Morphina (part.), Plötz, Mitt. Nat. Ver. Neu-Vorpomm., v. 37, p. 5 (1886). - Morphidae (part.), E. Haase, Corr:Bl. Ver. Tris, v. 1, p. 102, 106, 306 (1886 \& 88). - Morphinae (part.), Wood-Mason \& Nicéville, Journ. Asiat. Soc. Bengal, v. 55. II, 1886, p. 353 (1887). - Elwes \& Nicén ville, l. c., p. 419 (1887). - G. Semper: C. Semper, Reis. Archip. Philippin., pars 2. $\dot{V}$, p. 67 (1887). - L, Glaser, Cat. Etym. Col. Lep., p. 274, 286 ete. (1887). Morphina (Group) (part.) + Nymphalinae (part.), Distant \& Pryer, Ann. Mag. Nat. Hist., ser. 5, v. 19, p. 51 (1887). - "Morphiden", O. Staudinger (\& Schatz), Exot. Schmett., v. 1, p. 187 (1887). - Morphinae, A. Pagenstecher, Jahrb. Nassau. Ver. Naturk., v. 41, p. 91 (1888). - M. (part.), Elwes (\& Möller), Trans. Ent. Soc. London, n. ser., v. 4, p. 281, 331 (1S88). - M., Hampson, Journ. Asiat. Soc. Bengal, v. 57. II, 1888, p. 751 (1889). - "Morphiden“" ,Morphiden der alten Welt" (part.), (O. Staudinger) Röber \& Schatz, Exot. Schmett., v. 2, p. 181, 184 (18S9). Morphidae, Ribbe, Deutsch. Ent. Zeit. Lep., v. 2, p. 240 (1889). - Doherty, Journ. Asiat. Soc. Bengal, v. 58. IT, p. 124 (1889). - Morphinae, W. F. Kirby, Ann. Mag. Nat. Hist., ser. 6, v. 3, p. 160 (1889). - Morphides, Plateau (sec. E. Haase), Bull. Soc. Ent. Belgique, v. 33, p. LXI (1889). - Morphidae, Doherty: W. H. Holland, Proe. Boston Soc. Nat. Hist., v. 25, p. 58, 60 (1890). - Tiyon, 2. Ann. Rep. Admin. Brit. N. Guinea, App. 5, sep. p. 7 (1890). - Morphinae, Nicéville, Journ. Bombay Nat. Hist. Soc., v. 5, p. 296 (1890). - M. (part.), Manders, Trans. Ent. Soc. London, p. 519 (1890). - B. Hagen, Tijdschr. Nederl. Aardr. Gen., ser. 2, v. 7,p. 199 (1890). - $M$. (part.), E. Haase, Deutsch. Ent. Zeit. Lep., v. 4, p. 29, 33 (1891). - Elwes, Proc. Zool. Soc. London, p. 270 (1891). - Morphidae Doherty, Journ. Asiat. Soc. Bengal, v. 60. II, p. 26, 170 (1891). - Röber, Tijdschr. Ent., v. 34, p. 309 (1891). - M. (part.), W. Rothschild, Deutsch. Ent. Zeit. Lep., v. 5, p. 434 (1892). - Morphinae (part.), Leech, 
Butt. China, Jap. Corea, v. 1, p. 110 (1892). - Nicéville, Journ. Bombay Nat. Hist. Soc., v. 7, p. 325 (1892). Morphina, B. Hagen, Berlin. Ent. Zeit., v. 37, p. 144 (1892). - Morphinae (part.), Swinhoe, Trans, Ent. Soc. London, p. 275 (1893). - M., W. F. Kirby, Handb. Lep. (Allens Natural. Libr.), v, 1, p. XII, 193 (1894). - Nicéville, Journ. Asiat. Soc. Bengal, v. 63. II, p. 2 (1894). - M. (part.), id., Gazetteer Sikhim, List Butterfl., p. 130 (1894). M., Grose Smith, Nov. Zool., v. 1, p. 357 (1894). - Grose Smith \& W. F. Kirby, Rhop. Exot., v. 2, 3, to Tenaris 1-5 etc. (1894-98). - "Morfinos" (part.), Elera, Faun. Filipinas, v. 2, p. 269 (1895). - Morphinae (part.), Fürbringer (\& A. Pagenstecher): Semon, Zool. Forschungsreise i5: Denkschr. Med. Naturw. Ges. Jena, v. 8, p. (78) 238 (1895). - "Morphiden der alten Welt" (part.), E. Reuter, Acta Soc. Sci. Fenn., v. 22, p. 108 (1896). - Morphidae (Amathusiinae) (part.), L. Martin, Doutsch. Ent. Zoit. Lep., v. 8, 1895, p. 253 (1896). - Morphinae, A. Pagenstecher, Abh. Senckenb. Naturf. Gos., v. 23, p. 404 (1897). "Morphiden" (part.), Eimer (\& Fickert), Entstehung d. Arten, pars 2, Orthogen., p. 99 etc. sec. indic. (1897). Morphidae (part.), B. Fagen, Jahrb. Nassau. Ver. Naturk., v. 50, p. 79 (1897). - M., Ribbe, Deutsch. Ent. Zeit. Lep.. v. 11, p. 108 (1898). - Rebel, Termes, Füzetek, v. 21, p. 374 (1898). - M. (part.), R. Grote, Canad. Entom., v. 30, p. 203 (1898). - Morphinae (part.), K. Jordan, Nov. Zool., v. 5, p. 390 (1898). - "Morphiden", A. Pagenstecher, Zoologica, Heft 27, p. 92 (1899). - "Morphinen", id., Jahrb. Nassau. Ver. Naturk., v. 53, p. 109, 123 (distr. geogr.) (1900). - Morphinae (part.), Fruhstorfer, Berlin. Ent. Zeit., v. 45, p. $13(1900)$. - R. Grate, Ins.-Börse, v. 17, p. $243(1900)$. - W. J. Holland, Nov. Zool., v. 7, p. 62 (1900). - B. Hagen, Tijdschr. Nederl. Aardr. Gen,, ser. 2, v. 7, p. 199 (not. biol.) (1900). - M. (part.), W. F! Kirby, Hübner \& Geyer, Samml. Exot. Schmett., Neue Ausg.. v. 3, p. 46 (1901). - Morphidae, B. Hagen, Abh. Senckenb. Naturf. Ges., v. 20, p. 331 (1902). - Id.: A. Maaß, Bei liebensw ürd. Wilden, p. 208 (1902). - Fruhstorfer, Berlin. Ent. Zeit., v. 49, p. 189 (1904). - Morphinae, Bingham, Faun. Brit. Ind,, Lep., v. 1, p. 181 (1905). - Morphidae, Alfken, Abh. Naturw. Ver. Bremen, v. 18, p. 133 (1905). A. Seitz, Großschmett. Erde, v. 1, p. 155 (1908).

"Morphiden der alten Welt, Amathusiinen", A. Pagenstecher, Geogr. Verbr. Schmett., p. 412 (1909). - Morphinae, Bell, Journ. Bombay Nat. Hist. Soc., v. 19, p. 23, t. 1, f. 3 (larva); p. 35,467, 468, 469 (1909). - Morphidae, Strand, Deutsch. Ent. Zeit. Tris, v. 24, p. 193 (1910). - Hauser, Verh. Zool.-Botan. Ges. Wien, v. 60, p. (38) (1910). Morphinae (part.), G. H. C[arpenter], Encycl. Britann., ed. 11, v. 16, p. 477 (1911). - Morphidae, A. Pagenstecher, Abh. Senckenb. Naturf. Ges., $\nabla .33$, p. 411 (1911). - Strand. Mitteil. Zool. Mus. Berlin, v. 5, p. 470 (1911). - Rebel, Verh. Zool.-Botan. Ges. Wien, v. 61, p. (28) (1911). Morphonidae, Strand, Archiv f. Naturg., v. 79, 1913, Abt. A Heft 7, p. 183. - Morphinae (part.), Tytler, Journ. Bombay Nat. Hist. Soc., v. 21, p. 54 (1911). - W. H. Evans, l. c., v. 21, p. 571 (1912). - Morphidae, Eecke, Not. Leyden Mus., v. 35 , p. 243 (1913). - Id., 1. c., v. 36, p. 227 (1914). - Morphinae, Hannyngton, Journ. Bombay Nat. Hist. Soc., 
v. 24, p. 578 (1916). - M. V. Ellis, l. c., v. 2.5 p. 107 (1917) Morphinae, Ormiston, Spol. Zeylan., v. 11, p. 17 (1918). - Godfrey, Journ. Nat. Hist. Soc. Siam, v. 3, p. 467 (1919). - M. (part.), Ollenbach, Journ. Bombay Nat. Hist. Soc., v. 27, p. 887 (1921). - M., N. D. Riley \& Godfrey, Journ. Nat. Hist. Siam, v. 4, p. 173 (1921). Pendlobury, Journ. Feder. Malay Stat. Mus., v. 11, p. 34 (1923). - Ormiston, Butterfl. Ceylon, p. 17 (1924). Morphidae, Draeseke, Deutsch. Ent. Zeit. Iris, v. 39, p. 57 (1925). - Morphinae, F. X. Williams, Philipp. Journ. Sci., v. 35 , p. 74 (1925). - Morphonidae, Strand, Bull. Soc. Zool. Franco, v. 51, p. 402 (1926). - Morphidae, T. B. Fletcher, Bull. Agricult. Res. Inst. Pusa, Nr. 162, p. 575 (1926).

Th a u mantidae (part.), Bönninghausen, Verh. Ver. Naturw. Unterh. Hamburg, v. 19, p. 8 (1896).

A rginae (part.), G. F. Hampson, Nov. Zool., v. 25, p. '385 (1918). - Aeginae (error typ.), W. H. Evans (sec. Hampson), Journ. Bomb. Nat. Hist. Soc., v. 28, p. 32 (1921).

Brassolidae (incl. Amathusiidae), Thering, Ent. Rundschau, v. 46, p. 41 (1929).

\section{Subfamilia Amafhusiinae F. Moore}

A math us i ina e (Subfam., part.), F. Moore, Lep. Ind., v. 2, p. 170 (1893). - A., H. Stichel, Gen. Ins., fase. 36, p. 1 (1906). A. Seitz, Grolschmett. Erde, v. 1, p. 155 (1908). - A. (part.), Fruhstorfer: 1. c., v. 9, p. 403 (1911). - Amathusiidae (Fam.), H. Stichel, Tierreich, Lief. 34, p. 1 (1912). - Amathusiinae, W. Rothschild, Journ. Feder. Malay. Stat. Mus., v. 8, p. 153 (1920).

\section{Tribus Amathusiidi}

H. Stichel, Gen. Ins., fasc. 36, p. 7 (1906). - Id., Tierreich, Lief. 34, p. 5 (1912).

\section{Genus Amarhusia J. G. Fabr.}

J. C. Fabricius (MS): Illiger, Mag. Insektenk., v. 6, p. 279 (1807). - Latreille, Gen. Crust. Ins., v. 4, p. 194, 196 (1809). - A., Amathrsia (laps.!), Oken, Lehrb. Naturg., v. 3. I, p. XXV, 746 (1815). - Amathusia, Horsfield, Descr. Cat. Irep. E.-Ind. Comp., pars 1, p. 61 (1828). - G. R. Gray: Griffith-Cuvier, Anim. Kingdom, $\mathbf{y}_{\text {i }}$ 15, p. 590 (typ.: A. phidippus) (1832). - Boisduval (sec. Fabricius), Spec. Gến. Lép., v. 1, p. 79 (1836). - Id. (sec. Horsfield), 1. c., p. 153. - Id.: Cuvier, Règne Anim., ed. 3, v. 3, p. 251 (1836). - E. Blanchard, Hist. An. Artic., v. 3, p. 455 (1840). - Amathusa (!), Swainson \& Shuckard, Hist. Nat. Arrang. Ins. (Cab. Cycl. Lardner, v. 129), p. 93 (1840). - Amathusia, E. Doubleday, List Lep. Brit. Mus., pars 1, p. 114 (part.) (1844). - E. Blanchard, Hist. Ins., v. 2, p. 334 (1845). - E. Doubleday (\& Westwood), Gen. Diurn. Lep., v. 1, t. 54* (1849). - A. (Subgen.), Hoeven, Handb. Zool., v. 1, p. 398 (1850) - Amathusia (Thoraessa Boisduval MS), (part.), (E. Doubleday \&) J. O. Westwood, Gen. Diurn. Lep., v. 2, p. 327 (1851). - A., E. Blanchard (\& Doyère): Cuvier, Règne Anim., od. 4 (Crochard-Masson), v. 2, Ins., p. 246 (Typ.: Pap. phidippus). (1849).- Chenu \& H. Lucas, Fnc. Hist. Nat., Papill., p. 162 (1851). $\frac{-}{37}$ Ménétriés, Enum. Corp. Anim. Ac. Petropol., Lep., pars 1, p. 37 
(1855). - Horsfield \& F. Moore, Cat. Lep. Mus. E.-Ind. Comp., v. 1, p. 209 (part.) (1857). - Carus-Gerstäcker, Handb. Zool., v. 2, p. 231 (1863). - Herrich-Schäffer, Corr.-Bl. Zool.-Min. Ver. Regensb., v. 18 , p. 112 ; v. 19 , p. 88 (1865). - H. W. Bates, Journ. Ent., v. 2, p. 178 (1866). - A. G. Butler, Cat. Diurn. Lep. Fabr., p. 45 (part.) (1869). - Id., Trans, Ent. Soc. London, p. 481, 485 (1870). - W. F. Kirby, Cat. Diurn. Lep., p. 115, App.. p. 646 (1871). - S. H. Scudder, Proc. Amer. Ac. Sei., v. 10, pa 108 (1875). - Wallace, Geogr. Distr. Anim., v. 1, p. 319 (1876). Piepers (\& P. C. T. Snellen), Tijdschr. Int., v. 19, p. 147 (1876). - F. Müller, Jenai. Zcit. Naturw., v. 11, p. 101 (1877). - A. G. Butler, Trans. Linn. Soc. London, ser. 2, Zool., v. 1, p. 538 (1879). - W. F. Kirby, Scient. Proc. R. Dublin Soc., ser. 2, v. 2, p. 300 (part.) (1880) - C. Crüger (sec. Kirby), Berlin. Ent. Zeit., v. 25, p. 111 (1881). - Distant, Rhop. Malay., p. 69, 70, 423 (part.) (1882 \& 86). - G. F. L. Marshall \& Nicéville, Butt. Ind. Burm. Ceylon, v. 1. II, p. 282, 288 (part.) (1883). - E. Gerharr, Deutseh. Ent. Zeit., v. 27, p. 181 (distr. geogr.) (1883). - Kheil, Rhop. Nias, p. $20(1884)$. - Doherty, Journ. Asiat. Soc. Bengal, v. 55. II, p. 121 (1886). - E. Haase, Corr,-Bl. Ent. Ter. Iris, v. 1, p. "102, 307 (1886 \& 88). - Id., Zool. Anz., v. 11, p. 479 (org. odor.) (1888). L. Glaser, Cat. Etymol. Col. Lep., p. 274 (1887). - G. Semper: O. Semper, Reis. Arch. Philipp., pars 2. V, p. 70 (part.) (1887). O. Staudinger (\& Schatz), Exot. Schmett., v. 1, p. 187, 188 (1887). - Röber: O. Staudinger \& Schatz, Exot. Schmett., v. 2, t. 31 (Morphol.); p. 184, 185 (1888 \& 89). - Plateau (sec. E. Haase). Bull. Soc. Ent. Belgique, p. LXI (organ. odor.) (1889). - Skertchly, Ann. Mag. Nat. Hist., ser. 6, v. 3, p. 210, 212 (1889). W. J. Holland, Proc. Boston Soc. Nat. Hist., v. 25, p. 58 (1890). B. Hagen, Tijdschr. Nederl. Aardr. Gen., ser. 2, v. 7, p. 199 (part.) (1890). - Children (sec. Fabricius), Philos. Mag., v. 7, p. 118 (Typ. A. phidippus) (1890). - P. C. T. Snellen, Midden Sumatra, v. 4. VIII, p. 16 (1892). - W. F. Kirby, Handb. Lep. (Allens Natural. Libr.), v. 1, p. 198 (1894). - F. Moore, Lep. Ind., r. 2, p. 173, 178 ('Typ. A. phidippus) (1895). - Elera, Cat. Faun. Filippin., v. 2, p. 270 (part.) (1895). - E. Router, Acta Soc. Sci. Fenn., v. 22 , p. 109 (1896). - L. Martin, Deutsch. Ent. Zeit. Lep., v. 8, p. 258 (1896). - A. Pagenstecher, Abh. Senckenb. Naturf. Ges., v. 23, p. 404 (1897). - Fruhstorfer, Deutsch. Ent. Zuit. Lep., v. 12, p. 74 (1899). - H. Stichel, Deutsch. Ent. Zeit. Iris, v. 15, p. 59 (1902). - Röber, Ent. Zeit. Stettin, v. 64, p. 338 (1903). - L. C. H. Youug, Journ. Bombay Nat. Hist. Soc,, v. 15, p. 297 (1903). - Fruhstorfer, Deutsch. Ent. Zeit. Iris, v. 17, p. 152 (1901). - A., Sectio 1, Shelford, Journ. Straits Branch Asiat. Soc., Nr. 41, p. 107 (1904). A., Bingham, Faun. Brit. Ind., Butterfl., v. 1, p. 181, 187 (syn. part.) (Typ. A. phidippus) (1905). - H. Stichel, Gen. Ins., fasc. 36, p. 7, t. 1, f. 1 (Morphol.) (1906). - A. Pagenstecher, Geogr. Verbr. Schmett, p. 412 (1909). - Bell, Journ. Bombay Nat. Hist. Soc., v. 19, p. 469 (1909). - L. Martin, Mitt. Münch. Entom. Ges., v. 1, p. 71 (1910). - Strand, Iris 1910, p. 193. - Fruhstorfer: A. Seitz, Großschmett. Eide, v. 9, p. 426 (1911). - A. (Genus, Subgen.), W. H. Evans, Journ. Bombay Nat. Hist. Soc., v. 21, p. 571 (1912). - A. (Gen.), H. Stichel, Tierreich, Lief. 34, p. 4, 5, 1. 2, 3 (Morphol.) (1912). - Moulton, Journ. Straits Branch Asiat. Soc., Nr. 65, p. 13 (1913). - Eecke, Zool. Mededeel. Leiden, v. 4, p. 83 (1918). - W. H. Evans, Journ. Bombay Nat. Hist. Soc., v. 29 , p. 793,796, t. 16, E 7 (1923). Le Cerf, Enc. Ent., ser. B. 3, Lep., v. 1, p. 141 (Morphol.) (1926). - W. H. Evans, Identif. Ind. Butterfl., p. 84, 86, t. 16, E 7 (1927). 
Eque s [Subgen.] (part.), Linné, Syst. Nat., ed. 12, v. 1. II, p. 744 (1767). - Gmelin-Linné, Syst. Nat., ed. 13, v. 1. V, p. 2243 (1790). - E. (Achivus) (part.), D. H. Schneider, Nomencl. Entom., p. 33 (1785).

A chivus [Subgen.] (part.), P. L. S. Müller, Naturs. Linné, v. 5. I, p. 573 (1774).

P.terourus (part.), Scopoli, Introd. Hist. Nat., p. 433 (1777). - S. H. Scudder, Proc. Amer. Ac. Sci., v. 10, p. 259 (1875).

$N y m p h$ a lis (part.), Latreille, Gen. Crust. Ins., v. 4, p. 193, 194 (1809). - Id.; Cuvier, Règne Anim., v. 3, p. 544, 545 (1817).

Sa ty $r$ u s, "Satyre", Latreille, Consid. Gén., p. 355, 440 (1810). - Satyrus (Amathusia), Goldfuß, Handb. Zool., v. 1, p. XI, 578 (1820).

Moera (part.), Jac. Hübner, Verz. Schmett., p. 51 (1818). - S. H. Scudder, Proc. Amer. Ac. Sci., v. 10, p. 220 (nom, praeocc.) (1875).

Mor pho (part.), Latreille, Encycl. Méth., v. 9, p. 11, 435 $(1819 \& 24)$. - J. B. Godard, 1. o., p. 439 (1924). Boisduval (sec. Latreille), Spec. Gén. Lép., v. 1, p. 79, 118 (Typ. Pap. menelaus, phidippus) (1836). - Verloren, Cat. Ins. Lep. Cramer, v. 2, p. 200 (1837). - GuérinMéneville, Icon. Règne Anim. Cuvier, v. 7, Ins., p. 487 (1844). - M. (Amathusia, Subgen.), Hoeven, Handb. Zool., v. 1, p. $398(1850)$.

Mitoc er u s, Billberg, Enum. Ins., p. 79 (1820). - S. H. Scudder, Proc. Amer. Ac. Sci., v. 10, p. 220 (1875).

Pavonia, Amathusia, Griffith: Cuvier, Anim. Kingdom, v. 15 , p. $590(1832)$. - F. S. Voigt-Cuvier, ut antea, v. 5, p. $535(1839)$.

$P$ seudamathusia, Honrath, Corr.-Bl. Ent. Ver. Iris, v. 1, p. 91 (1886). - P. (Subgen.), O. Staudinger (\& Schatz), Exot. Schmett., W. J. Holland, Proc. Boston Soc. Nat. Hist., v. 25, p. 59 (1890). - P., F. Moore, Irep. Ind., v. 2, p. 182 (1895). - H. Stichel, Deutsch. Ent. Zeit. Iris, v. 15, p. 59 (nota) (1902). - Röber, Ent. Zeit. Stettin, v. 64, p. 338 (nom. infirm.) (1903). - Fruhstorfer, Deutsch. Ent. Zoit. Lep., v. 12, p. 77 (1899). - P. (Sectio), Shelford, Journ. Straits Branch Asiat. Soc., Nr. 41, p. 107 (1904). $-P$. (Subgen.); Fruhstorfer, Deutsch. Ent. Zeit. Iris, v. 17, p. 157 (1904). - P. (Artengruppe), id.: A. Seitz, Großschmett. Ende, v. 9, p. 426, 431 (1911).

\section{Sectio Afegana}

H. Stichel, Gen. Ins., fasc. 36, p. 10 (1906). - Id., Tierreich, Lief. 34 , p. 9 (1912).

H. Stichel, ut supra (1906 \& 12).

Cohors a. Phidippiformes

phidippus (Johans.) Papilio p., Johansson: Linné, Burma, Amoen. Acad., v. 6, p. 402 (1764). - Amathusia p., Distant, Rhop. Malay., p. 70, 71 (part $\lambda)$; p. 67, f. 17, 18 (larva) (1882 \& 86). - Marshall \& Nicéville, Butt. Ind., Burmah, Ceyl., v. 1. II, p. 289 (except.

Siam,

Malayische

Halbinsel,

Mergui,

Malay. 
larvae descr., vide Discophora celinde) (1883). - F. Moore, Lep. Ind., v. 2, p. 179 (in cit.) (1895). - Fruhstorfer, Deutsch. Ent. Zeit. Lep., v. 12, p. 74 (1899). - H. Stichel, Gen. Ins., fasc. 36, p. 10 (part.) (1906). - Fruhstorfer: A. Seitz, Großschmett. Ende, v. 9, p. 426 (1911) - - H. Stichel, Tierreich, Lief. 34, p. 8, 9 (distrib. corrig.) (1912). Eecke, Zool. Mededeel., v. 4, p. 83 (1918).Godfrey, Journ. Siam Soc. Nat. Hist., Suppl., v. 7, p. 259 (1930).

- subsp. phidippus (Johans.), Papilio p., Johansson: Linné ut supra (1764). $-P$. ,Javaensis", Seba, Thesaurus, v. 4, p. 13, t. 5 , f. 5 (1765). - P. (Eques) p., Linné, Syst. Nat., ed. 12, v. 1. II, p. 752 (1767). P. (Achivus) p., P. L. S. Müller, Naturs. Linné, v. 5. I, p. 577 (1774). - P. p., J. C. Fabricius, Syst. Ent., p. 455 (1775). (P. F. Gmelin \&) Christmann, Onomat. Med., v. 6, p. 123 (1775). — "Phidippres", Pap. p., P. Cramer, Pap. Exot., v. 1, p. 108, t. 69 , f. A, B; p. $154(1775$ \& 76). Pterourus p., Scopoli, Introd. Hist. Nat., p. 433 (1777). - Papilio p., Goeze, Ent. Beytr., v. 3. I, p. 64 (1779). - J. C. Fabricius, Spec. Ins., v. 2, p. 21 (1781). - C. Stoll, Essai Syst. Lep.: P. Oramer, Pap. Exot., v. 4, Append., p. 9 (1782). - J. C. Fabricius, Mant. Ins., v. 2, p. 11 (1787). - (Jablonsky \&) Herbst, Naturs. Ins., Schmett., v. 3, p. 102, t. 35, f. 3, 4 (1788). - P. (Eques) p., F. G. Gmelin-Linné, Syst. Nat., ed. 13, v. 1. V, p. $2243(1790) .-P$. p., C. Jung, Alphab. Verz. Schmett., v. 2, p. 101 (1792). - J. C. Fabricius, Ent. Syst., v. 3. I, p. 71 (1793). - Latham \& H. Davies: J. A. Forster, Zool. Index, ed. 2, pars 3, p. 23 (1795). - Esper, Ausl. Schmett., t. 56 , f. 1 (1796). - Turton, Gen. Syst. Nat., v. 3. II, p. 82 (1806). Amathusia p., J. C. Fabricius MS.: Illiger, Mag. Insektenk., v. 6, p. 279 (1807). - A. (Papilio) p., Iatreille, Gen. Crust. Ins., v. 4, p. 196 (1809). - "Satyre" (P.) p., id., Consid. Gén., p. 440" (1810). - Amathrsia (err. typ.), Oken, Lehrb. Naturg., v. 3. I, p. 746 (1815). - Nymphalis (Papilio) p., Latreille: Cuvier, Règne Anim., v. 3, p. 545 (1817). - Moera phidippe, Jac. Hübner, Verz. Schmett., p. 51 [1818]. - Satyrus p., Goldfuß, Handb. Zool., v. 1, p. 578 (1820). - Morpho p., (Latreille \&) J. B. Godart, Enc. Méth., v. 9, p. 439 (1824). - Amathusia $p_{0}$, Horsfiold, Descr, Cat. Lep. Mus. E.-Ind. Comp., pars 2, t. 7 , f. $10,10 \mathrm{a}-\mathrm{h}$ (larva, append.) (1829). - Morpho p., Guérin-Méneville, Iconogr. Règne Anim., Cuvier, v. 7 , Ins., t. 79 , f. $3,3 \mathrm{a}-\mathrm{d}$ (larva

Archipel (östlich bis Celebes und Mindanao), Andamanen, Nicobaren, ? BritischOstindien

West-Java 
etc.), p. 487 (1829-44). - Pavonia, Ama-

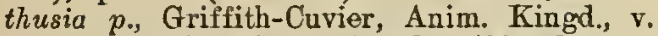
15, p. 590 (1832). - Papilio (Morpho) p., Boisduval (cec. Latreille), Spec. Gén. Lép., v. 1, p. 118 (1836). - Amathusia p., id.: Cuvier, Règne Anim., ed. 3, v. 3, p. 251 (1836). - Morpho p., Verloren. Cat. Syst. Lep. Cramer, v. 1, p. 38; v. 2, p. 201 (1837). - Amathusia p., E. Blanchard, Hist. An. Artic., v. 3, p. 455 (1840). - E. Doubleday, List Lep. Brit. Mus., v. 1, p. 114 (1844). - E. Doubleday (\& Westwood), Gen. Diurn. Lep., v. 1, t. 54, f. 2 (1849). - E. Blanchard (\& Doyère): Cuvier Règne Anim., ed. 4 (Crochard-Masson), v. 2, p. 327 (1849). - A. p. (Thoraessa p. Boisduval MS.), (E. Doublexlay \&) J. O. Westwood, Gen. Diurn. Lep., v. 2, p. 327 (1851). - A. p., Chenu \& H. Lucas, Enc. Hist. Nat., Papill., p. 162 , t. 37, f. 1 (1851). - Ménétriés, Enum. Corp. Anim. Ac. Petropol., pars 1, p. 37 (1855). - J. O. Westwood, Trans. Ent. Soc. London, n. ser., v. 4, p. 164 (larva sec. Horsfield) (1856). - Horsfield \& F. Moore, Cat. Lep. Mus. E.-Ind. Comp., v. 1, p. 209, t. 6, f. 4, 4a (larva, chrysil.) (1857). Herrich-Schäffer, Corr.-Bl. Zool.-Min. Ver. Regensb., v. 19, p. 88 (1865). - A. G. Butler, Cat. Diurn. Lep. Fabricius, p. 45 (1869). - W. F. Kirby, Trans. Ent. Soc. London, p. 137 (recogn. typ.) (1870). - Id., Cat. Diurn. Lep., p. 115 (1871). - Piepers, Tijdschr. Entom., v. 19, Verslag, p. XXI (1876). - Piepers (\& P. C. T. Snellen), Tijdschr. Entom., v. 19, p. 147 (Biol.) (1876). - C. A. Dohrn (sec. Piepers), Ent. Zeit. Stettin, v. 37, p. 443, 444 (part.) (1876). - P. C. T. Snellen, Tijdschr. Entom., v. 20 , p. 66 (1877). - W. F. Kirby, Entomologist, v. 10, p. 291 (1877). - Id., Scient. Proc. R. Dublin Soc., ser. 2, v. 2, p. 300 (1880). - O. Staudinger (\& Sehatz), Exot. Schmett., v. 1, t. 63, p. 187 (distr. part.) (1886 \& 87). - W. Müller, Zool. Jahrb., Syst., v. 1, p. 611 (Biol.) (1886). - E. Haase, Corr.-Bl. Ent. Ver. Iris, v. 1, p. 308 (org. odor.) (1888). - W. F. Kirby, Handb. Lep. (Allens Nat. Libr.), v. 1, p. 194 (1894). - Fürbringer (\& A. Pagenstecher): Semon, Zool. Forschungsreise: Denkschr. Med.-Naturw. Ges. Jena, v. 8, p. 238 (1895).

E. Reuter, Acta Soc. Sci. Fenn., v. 22, p. i09 (1896). - Piepers, 3. Congr. Internat. Zool., 1895 , p. 464 (Mimicry) (1896). - Fruhstorfer, Berlin. Ent. Zeit., v. 41, 1896, p. 301 (1897). - Eimer (\& Fickert), Entstehung d. Arten, pars 2, Orthogen., p. 99 etc. sec. indic., f. 134 (1897). - Fruhstorfer, Deutsch. Ent. Zeit. Lep., v. 12, p. 74 (part.) (1899). 
- A. p. p., id., Deutseh. Ent. Zeit. Iris, v. 17, p. 156 (part.) (1904). - H. Stichel, Gen. Ins., fasc. 36, p. 10 (cit. part.) (1906). - Fruhstorfer: A. Seitz, Großschmett. Ende, v. 9, p. 428 (1911). - G. H. C [arpenter], Encycl. Britann., ed. 11, p. 478, f. 71 (larva) (1911). - A. p. p. forma principalis, $H$. Stichel, Tierreich, Lief. 34, p. 8, 12, 13 (cit. corrigend.) (1912). - A. p., W. H. Evans, Journ. Bombay Nat. Hist. Soc., v. 22, 1913, p. 763 (1914).

forma retrog ad a Frust. A. p. p. forma r., Typ.: Java Fruhstorfer: A. Seitz, Großschmett. Erde, v. 9, p. 428 (Trockenzeitform) (1911). H. Stichel, Tierreich, Lief. 34, p. 11, 13 (1912).

subsp. majada Fruhst. A. p. m., Fruhstorfer, Soc. Entom., v. 31, p. 24 (1916).

A. $p h$ idippu 8 , A. Pagenstecher, Jahrb. Nassau. Ver. Naturk., v. 43, p. 96 (1890). ? P. O. T. Snellen, Tijdschr. Entom., v. 45, p. 81 (1902).

- subsp. baweanica Fruhst. A. p. baweani- Bawean cus, Fruhstorfer, Soc. Entom., v. 20, p. 129; p. 140, fig. (1905). - Id., Ins.-Börse, v. 23, p. 95 (nota) (1906). - A. p. baweanica, $\mathrm{H}$. Stichel, Gen. Ins., fasc. 36, p. 58 (1906). Fruhstorfer: A. Seitz, Großschmett. Erde, v. 9, p. 428 (1911).

A. $p$ h id i $p$ p $и$ s (part.), H. Stichel, Tierreich, Lief. 34, p. 12, 13 (1912).

subsp. lo m bo li i a na Fruhst. A. p. l., Fruh- Iombok, storfer, Soc. Entom., v. 20, p. 130 (1905). ? Bali - H. Stichel, Gen. Ins., fasc. 36, p. 59 (1906). - Fruhstorfer: A. Seitz, GroBschmett. Erde, v. 9, p. 428 (1911).

A. $p h i d i p p u s$, Fruhstorfer, Berlin. Ent. Zeit., v. 42, p. 4 (1897). - Nicéville \& Elwes, Journ. Asiat. Soc. Bengal, v. 66. II, 1897, p. 681 (? part.) (1898). - A. p. p. (part.), H. Stichel, Tierroich, Lief. 34, p. 12 (1912).

subsp. e utropius Fruhst. A. p. e., Fruh- Sumatra, storfer: A. Seitz, Großschmett. Erde, v. 9, Simalur, p. 427 (1911). - Eecke, Zool. Mededeel. Lei- ? Billiton, den, v. 4, p. 83 (1918).

? A. phidippus, F. D. Godman \& O. Salvin, Bangka Proc. Zool. Soc. London, p. 638 (1878). P. C. T. Snellen, Tijdschr. Entom., v. 33, p. 217 (1890). - ? Id́., 1. c., p. 291 (1890). - B. Hagen, Tijdschr. Nederl. Aardr. Gen. ser. 2, v. 7, p. 199 (not. biol.) (1890). ? P. C. T. Snellen, Not. Leyden Mus., v. 13, p. 136 (not. biol.) (1891). - Id., Mridden Sumatra, v. 4. VIII, p. 16 (1892). - B. Hagen, Berlin. Ent. Zeit., v. 37, p. 144 (1892). - Nicéville \& L. Martin, Journ. Asiat. Soc. Bengal, v. 64 . II, 1895 , p. 393 (not. biol.) (1896). - L. Martin, Deutsch. 
Ent. Zeit. Lep., v. 8, 1895, p. 258 (not. biol.); p. 260 (aberr.) (1896). - Strand, Deutsch. Ent. Zeit. Iris, v. 24, p. 193 (1910). - A. p. p., H. Stichel, Tierreich, Lief. 34, p. 15 (1912).

forma retracta Fruhst. A. p. eutropius forma $r$, Fruhstorfer: $\Lambda$. Seitz, Großschmett. Erde, v. 9, p. 428, t. 105 a (1911).

A. phidippus p., H. Stichel, Tierreich, Lief. 34, p. 12, 13 (part.) (1912).

subsp. nia $s$ a n a Fruhst. A. $p$. n., Fruhstorfer, Soc. Entom., v. 14, p. 2 (1899). - Id., Deutsch. Ent. Zeit. Lep., v. 12, p. 71, 75 (1899). - H. Stichel, Geu. Ins., fase. 36, p. 11 (1906). - Fruhstorfer: A. Seitz, Großschmett. Erde, v. 9, p. 428 (1911). H. Stichel, Tierreich, Lief. 34, p. 11, 15 (1912).

A. phidippus. Kheil, Rhop. Nias, p. 20 (not. biol.) (1884). - ? B. Hagen, Bei liebenswürd. Wilden, Anhang, p. 208 (1902). - ? Id., Abh. Senckenb. Naturf. Ges., v. 20, p. 331 (1902).

subsp. melanops Eecke A. p. m., Eecke, Pulu Lasia Zool. Mededeel. Leiden, v. 4, p. 83, t. 8, f. 2 (p. Simular) (1918).

subsp. chersias Fruhst. A. p. c., Fruh- Malakka storfer: A. Seitz, Großschmett. Erde, v. 9, (Halbinsel p. 427 (1911). - A. p. p. forma c., H. Sti- Siam, chel, Ticrreich, Licf. 34, p. 11, 14 (1912). - Porak, A. p. c., Pendlebury, Journ. Fedcr. Malay Singapore) Stat. Mus., v. 11, p. 31 (1923). - Godfrey, Journ. Siam. Soc. Nat. Hist., v. 7, p. 260 (1930).

A. $p h i d i p p u s$, A. G. Butler, Trans. Linn. Soc. London, Zool., v. 1, p. 538 (1879). Distant, Rhop. Malay, p. 70, 71, 423 (part.) (1882 \& 86). - T. Moore, Lep. Ind., v. 2, p. 179 (part.) (1895). - ? Elwes (\& 0. Möller), Trans. Ent. Soc. London, p. 282 (1888).

subsp. a dus t a ta Fruhst. A. p. adustatus, Siam, Fruhstorfer, Deutsch. Ent. Zeit. Iris, v. 17, p. 152,156 (1904). - A. p. p. forma a., H. Stichel, Gen. Ins., fasc. 36, p. 11 (1906). - A. p. a., Fruhstorfer: A. Seitz, Großschmett. Erde, v. 9, p. 427 (1911). - A. p. $p$. forma a., H. Stichel, Tierreich, Lief. 34, p. 12, 14 (1912). - A. p. a., Godfrey; Journ. Nat. Hist. Siam. v .2, p. 124 (1916). - Id., Journ. Siam Soc. Nat. Hist. Suppl., v. 7, p. $259(1930)$.

A. phidippus, Herb. Druce, Proc. Zool. Soc. London, p. 104 (1874). - F. Moore, 1. o., p. 826 (1878). - ? Id., Journ. Linn. Soc. London, Zool., v. 21, p. 33 (1886). Elwes \& Nicéville, Journ. Asiat. Soc. Bengal, v. 55. II, 1886, p. 419 (1887). - F. Moore, Lep.

\section{Burma, \\ Tenasserim}

Nias,

? Mentawai 
Ind., v. 2, p. 179 (part.), t. 146, f. 1, $1 \mathrm{a}_{\mathrm{r}}$ b (1895). - ? J. J. Walker, Trans. Ent. Soc. London, p. 450 (? patria falsa: Hongkong) (1895). - A. (A.) p., W. H. Evans, Journ. Bombay Nat. Hist. Soc., v. 21, p. 571 (? part.) (1912). - A. p., Ollenbach, l. c., v. 27, p. 887 (1921). - Godfrey, Journ. Siam Soc. Nat. Hist. Suppl., v. 7, p. 259 (part.) (1930).

forma Friderici Fruhst. A. p. f., Fruh- Typ.: storfer, Deutsch. Ent. Zeit. Iris, v. 17, p. 153, 156 (1904). - A. p. p. forma f., H. Stichel, Gen. Ins., fasc. 36 , p. 11 (cit. part.) (1906). - A. p. f., Fruhstorfer: A. Seitz, Großsohmett. Erido, v. 9, p. 427 (1911). - A. p. $p$. forma $f ., H$. Stichel, Tierreich, Lief. 34 , p. 11,14 (1912). - A. p. ,moc" f., W. H. Evans, Journ. Bombay Nat. Hist. Soc., v. 22,1013 , p. 763 (1914). - A. p. f., id., l. c., v. 29 , p. 796 (part.), t. 16, E 7. 1 (1923). Id., Identif. Ind. Butterfl, p. 86 (part.), t. 16, fig. E 7. 1 (1927). - E. p. ,race fredericki, Tytler, Journ. Bombay Nat. Hist. Soc., v. 31, p. 579 (1927).

A. $p h i d i p p u s$ var., F. Moore, Lep. Ind., v. 2 , p. 181, t. 147 , f. 1 ( ( ) (1895).

A. $p$ hidippus perakana (part.), (non A. perakana Honr.), Fruhstorfer, Deutsch. Ent. Zeit. Lep., v. 12, p. 75 ( I899).

subsp. (?) innominata. A. phidippus, Ferguson, Journ. Bombay Nat. Hist. Soc., v. 6, p. 437 (not. biol.) (1891). - A. (A.) p., W. H. Evans, 1. c., v. 21, p. 571 (part.), (1912).

A. $p h i d$. friderici (non Fruhstorfer), W. H. Evans, Journ. Bombay Nat. Hist. Soc., v. 29, p. 796 (part.) (1923). - Id., Identif. Ind. Butterfl., p. 86 (part.) (1927). subsp. andamancnsis Fruhst. A. taenia a., Fruhstorfer, Deutsch. Ent. Zeit. Lep., v. 12, p. 76 (1899). - A. phidiprpus a., id., Deutsch. Ent. Zoit. Iris, v. 17, p. 156 (forma tempest.) (1904). - H. Stichel, Gen. Ins., fasc. 36, p, 11 (1906). - Fruhstorfer: A. Seitz, Großschmett. Ende, v. 9, p. 427 (1911). - H. Stichel, Tierreich, Lief. 34, p. 11,15 (1912). - A. (A.) p. a., W. H. Evans, Journ. Bombay Nat. Hist. Soc., v. 21, p. 571 (1912). - A. p. andamanica (!), id., l. c., v. 29, p. 796 (1923). - Id, Identif. Ind. Butterfl., p. 86 (1923).

A. $p \hbar$ idippus, Hewitson, Ann. Mag. Nat. Hist., ser. 4, v. 14, p. 356 (1874). - F. Moore, Proc. Zool. Soc. London, p. 583 (1877). - A. p. "Andaman Form", id., Lep. Ind., v. 2 , p. 181 , t. 147 , f. 2 (ㅇ) (1905). - A. p., Wood-Mason \& Nicéville, Journ. Asiat. Soc. Bengal, v. 50. II, p. 245 (1881).

Britisch-

(Travancore, Bassein) 
subsp. cariatincta H. Stich. A. p. p. Natuna forma c., H. Stichel, Gen. Ins., fasc. 36, p. 11 (1906). - A. p. c., Fruhstorfer: A. Seitz, Großschmett. Erde, v. 9, p. 428 (1911). - A. p. p. forma c., H. Stichel, Tierreich, Lief. 34, p. 11, 14 (1912). - A. c. (A. phidippus local form), Moulton, Journ. Straits Branch Asiat. Soc., Nr. 65, p. 14 (1913).

- subsp. diluta Fruhst. A. p. dilutus, Fruhstorfer, Deutsch. Ent. Zeit. Iris, v. 17, p. 152,156 (1904). - A. p. p. forma diluta, H. Stichel, Gen. Ins., fasc. 36, p. 10 (1906). - A. p. dilutus, Fruhstorfer; A. Seitz, Großschmett. Erde, v. 9, p. 428 (1911).A. p. p. forma diluta, H. 'Stichel, Tierreich, Lief. $3 \pm$, p. 11, 14 (1912). - A. p. forma dilutus, Moulton, Journ. Straits Branch Asiat. Soc., Nr. 65, p. 14 (1913).

A. $p h i d i p p u s$, Herb. Druce, Proc. Zool. Soc. London, p. 104 (1873). - Distant \& Pryer, Aun. Mlag. Nat. Hist., ser. 5, v. 19, p. 51 (1887). - Skertchly, 1. c., ser. 6, v. 4, p. 212 (not. biol.) (1889). - A. G. Butler, Proc. Zool. Soc. London, p. 120 (1892). - Shelford, Journ. Straits Branch Asiat. Soc., Nr. 41, p. 107 (part.) (1904). Moulton, Ent. Monthly Mag., v. 48, p. 81 (Mimicry) (1912). - Id., Journ. Straits Branch Asiat. Soc., Nr. 65, p. 107 (1913).

forma e pides ma Fruhst. A. p. dilutus for- Typ.: ma e., Fruhstorfer: A. Seitz, Großschmett. SüdostFrde, v. 9, p. 428 (ㅇ). (1911). - A. p. p. Borneo forma e., H. Stichel, Tierreich, Lief. 34, p. 12,14 (1912).

forma arrenopia Fruhst. A. p. dilutus O- Typ.: forma a., Fruhstorfer: A. Seitz, Großschmett. Erde, v. 9, p. 428 (1911). - A. p. p. forma a. H. Stichel, 'Tierreich, Lief. 34 , p. 12, 14 (1912).

- subsp. celebensis Fruhst. A. p. c., Fruhstorfer,. Soc. Entom., v. 14, p. 2 (1899). Id., Deutsch. Ent. Zeit. Lep., v. 12, p. 72, 76 (1899). - A. p. var. c., Röber, Ent. Nachr., v. 26, p. 202 (1900). - A. p. c., H. Stichel, Gen. Ins., fasc. 36, p. 11 (1906). Fruhstorfor: A. Seitz, Großschmett. Erde, v. 9 , p. 429 , t. $105 \mathrm{a}$ (1911). - H. Stichel, Tierreich, Lief. 34, p. 12, 16 (1912).

A. $p h i d i p p u s$, Hopffer, Ent. Zeit. Stettin, v. 35, p. 38 (1874). - P. C. T. Snellen, Tijdschr. Entom., v. 21, p. 11 (1878). Holland, Proc. Boston Soc. Nat. Hist., v, 25, p. 58 (1890). - A. Pagenstecher, Abh. Senckenb. Naturf. Ges., v. 23, p. 404 (cit. except.) (1897).

A. virgata (non Butler), O. Staudinger (\& Schatz), Bxot. Schmett., v. 1, p. 188 (1887). - A. phidippus var. virgatus, W. Rothschild, Deutsch. Ent. Zeit. Lep., v. 5, p. 434 (1892). 
subsp. $K \ddot{u} h n i$ Röb. A. k., Röber, Ent. Nachr., Bangkai v. 26, p. 201 (1900). - A. phidippus k., (nördl. Fruhstorfer, Deutsch. Int. Zeit. Iris, v. 17, Colebes) p. 156 (1904). - H. Stichel, Gen. Ins., fasc. 36, p. 11 (1906). - Fruhstorfer; A. Seitz, Großschmett. Erde, v. 9, p. 429 (1911). - H. Stichel, Tierreich, Lief. 34, p. 12,16 (1912).

subsp. p a la wana Fruhst. A. p. palawanus, Palawan

Fruhstorfer, Deutsch. Ent. Zeit. Iris, v. 17, p. 154,156 (1904). - A. p. palawana, H. Stichel, Gen. Ins., fasc. 36, p. 12 (1906). - Fruhstorfer: A. Seitz, Großschmett. Erde, v. 9, p. 428 (1911). - H. Stichel, Tierreich, Jief. 34, p. 12, 17 (1912).

A. $p h i d i p p u s$, O. Staudinger, Deutsch. Ent. Zeit. Lep., v. 2, p. 40 (1889).

subsp. pollicaris Butl. A. p., A. G. But- Philippinen ler, Trans. Ent. Soc. London, p. 485 (1870). - O. Staudinger (\& Schatz), Exot. Schmett., v. 1, p. 187 (1887). - W. F. Kirby, Cat. Diurn. Lep., App., p. 646 (1871). - F. Moore, Lep. Ind., v. 2, p. 182 (1895). A. phidippus pollicaris, Fruhstorfer, Deutsch. Ent. Zeit. Lep., v. 12, p. 75 (1899). - H. Stichel, Gen. Ins., fasc. 36, p. 11 (1906). Fruhstorfer: A. Seitz, Großschmett. Erde, v. 9, p. 429 (1911). - H. Stichel, Tierreich, Lief. 34, p. 12, 17 (1912). - N. D. Riley \& Gabriel, Cat. Type Spec. Rhop. Brit. Mus., pars 1, p. 47 (1924).

A. phidippus, O. Staudinger (\& Schatz), Exot. Schmett., v. 1, p. 188 (1887). - G. Semper: C. Semper, Reis. Arch. Philipp., pars 2. V, Schmett., v. 1, p. 71 (cit. part.); p. 332 (1887 \& 92). - Elera, Cat. Faun. Filipinas, v. 2, p. 270 (cit. except., distr. part.) (1895). - F. X. Williams, Philipp. Journ. Sci., v. 35, p. 74 (Biol.), t. 2, f. 1; t. 3 , f. 3 (chrys., imago) (1924).

Binghami Fruhst. A. phidippus b., Fruhstorfer, Malakka Deutsch. Ent. Zeit. Iris, v. 17, p. 154, 156 (forma temp. sic. + forma temp. pluv.) (1904). - A. p. p. forma b., H. Stichel, Gen. Ins., fasc. 36 , p. 11 (1906). - A. b., Fruhstorfer: A. Seitz, Großschmett. Erde, v. 9, p. 429, t. $105 a$ : (schönbergi) (1911). - H. Stichel, Tierreich, Ijief. 34, p. 8, 17 (1912). - A. b. (A. phidippus local form), Moulton, Journ. Straits Branch Asiat. Soc., Nr. 65, p. 14 (1913). - ? A. p. b., Tytler, Journ. Bombay Nat. Hist. Soc., v. 31, p. 579 (1927).

A. $p h i d i p p u s$, Distant, Rhop. Malay., p. 70 (part.) (conf. cit. A. phidipp. i. spec.), t. 6 , f. $6,7\left(\sigma^{7}, 9\right)$ (sec. Fruhstorfer, 1904) (1882). - G. Semper: C. Semper, Reis.

(Penang,

Perak),

West-

Sumatra,

? Mergui

(sec. Tytler) Arch., Philipp., pars 2. V, p. 71 (in cit.) 
(1887). - F. Moore, Lep. Ind., v. 2, p. 179 (part.) (1895).

A. phidippus perakana (non Honrath), Fruhstorfer, Deutsch. Ent. Zeit. Lep., v. 12, p. 75 (part.) (1899).

A. $s$ c honbergi (non Honrath), Fruhstorfer:

A. Seitz, Grolschmett. Erde, v. 9, t. 105 a (corr. p. 1135) (1911, 1927).

Schönbergi Honr. A. 8., Honrath, Berlin. Ent. Zeit., Malayische v. 31, 1887, p. 347 (part.) (1888). - Shel- Halbinsel, ford, Journ. Straits Branch Asiat. Soc., Nr. Sumatra, 41, p. 107 (1904). - H. Stichel, Gen. Ins., Borneo fasc. 36, p. 12 (1906). $-A . s .+A$. ochraceofusca, Frubstorfer: A. Seitz, Großschmett. v. 9, p. 429 (1911). - A. s., H. Stichel, Tierreich, Lief. 34, p. 8, 18 (1912).

subsp. Sch ön bergi Honr. A. 8., Honrath, Perak, ut antea, t. 6 , f. 1 (1888). - A. schonber- Sumatra gii, F. Moore, Tep. Ind., v. 2, p. 182 (1895). - A. schönbergi, L. Martin, Deutsch. Ent. Zeit. Lop., v. 8, p. 259 (1896). — Nicéville \& L. Martin, Journ. Asiat. Soc. Bengal, v. 64. II, 1895, p. 394 (1896). - Fruhstorfer, Deutsch. Ent. Zeit. Lep., v. 12, p. 76 (part.) (1899). - Röber, Ent. Nachr., v. 26, p. 202 (1900). - A. s. s., H. Stichel, Gen. Ins., fasc. 36, p. 12 (1906). - A. s., Strand, Deutsch. Ent. Zeit. Iris, v. 24, p. 193 (1910). - A. s. 8., II. Fruhstorfer: A. Seitz, Großschmett. Erde, v. 9, p. 429 (non t. 105 a: = binghami).(1911). - A. s. s. forma principalis, $H$. Stichel, Tierreich, Lief. 34 , p. 19 (cit. part.), p. 20 (1912). - A. s., N. D. Riley \& Gabriel, Cat. Type Spec. Rhop. Brit. Mus., pars 1, p. 52 (1924).

forma ochrace of usca Honr. A. o., Hon- Typ.: Perak rath, Berlin. Ent. Zeit., v. 31, 1887, p. 348 (1888). - F. Moore, Lep. Ind., v. 2, p. 182 (1895). - L. Martin, Deutsch. Ent. Zeit. Lep., v. 8, p. 260 (1896). - A. phidippus var. •o., Nicéville \& L. Martin, 'Journ. Asiat. Soc. Bengal, v. 64. II, 1895, p. 394 (1896). - A. ochreofusca (!), Shelford, Journ. Straits Branch $A$ siat. Soc,, Nr. 41, p. 107 (part.) (1904). - A. ochrace of usca, Fruhstorfer, Deutsch. Ent. Zeit. Iris, v. 17, p. 155, 157 (1904). - A. schönbergi s. forma o., H. Stichel, Gen. Ins, fasc. 36, p. 12 (1906). - A. o. o., Fruhstorfer: A. Seitz, Großschmett. Erde, v. 9, p. 429 (1911). A. schönbergi s. forma o., H. Stichel, Tierreich, Lief. 34, p. 19, 20 (1912). - A. o., N. D. Riley, Cat. Type Spec. Rhop. Brit. Mus., pars 1, p. 40 (1924).

subsp. borneensis Fruhst. A. s. b., Fruh- Borneo storfer, Deutsch. Ent. Zeit. Lep., v. 12, p. 72,76 (1899). - Shelford, Journ. Straits Branch Asiat. Soc., Nr. 41, p. 107 (1904). 
- Fruhstorfer, Deutsch. Ent. Zeit. Iris, v. 17, p. 157 (1904). - H. Stichel, Gen. Ins., fasc. 36, p. 12,59 (1906). - Fruhstorfer: A. Seitz, Großschmett. Erde, v. 9, p. 430 (1911). - A. s. b. forma principalis, $H$. Stichel, Tierreich, Lief. 34, p. 19, 20 (1912). - A. s. b., Moulton, Journ. Straits Branch Asiat. Soc., Nr. 65, p. 107 (1913).

A. ochreofusca (non A. ochraceofusca Honr.), Shelford, Journ. Straits Asiat. Soc., Nr. 41, p. 107 (? part., an A. masina) (1904).

forma gabriel a Fruhst. A. ochraceofusca g., Fruhstorfer, Soc. Entom., v. 20, p. 130 (1905). - A. schönbergi borneensis forma g., H. Stichel, Gen. Ins., fass. 36, p. 59 (1906). - A. ochraceofusca g., Fruhstorfer: A. Seitz, Großschmett., v. 9, p. 430, t. 105 a (1911). - A. schönbergi borneensis forma g., H. Stichel, Tierreich, Lief. 34, p. 19, 20 (1912). - Moulton, Journ. Straits Branch Asiat. Soc., Nr. 65, p. 14 (1913).

patalena Westw., spec. dubia. A. p., Westwood, Cabin. Orient. Entom., p. 20, t. 19, f. 3 (sec. Westwood, 1851: p. 40, t. 19, f. 1) (1848). - (E. Doubleday \&) Westwood, Gen. Diurn. Lep., v. 2, p. 327 (1851). - A. phidippus var. A. platalena, Horsfield \& F. Moore, Cat. Lep. Mus. E.-Ind. Comp., v. 1, p. 210 (1857). - W. F. Kirby, Cat. Diurn. Lep., p. 115 (1871). - A. patalena, O. Staudinger (\& Schatz), Exot. Schmett., v. 1, p. 187 (A. phidippus aberr.) (1887). - A. phid. patalena, Fruhstorfer, Deutsch. Ent. Zeit. Lep., v. 12, p. 74, 76 (1899). A. patalana (!), id., Deutsch. Ent. Zeit. Iris, v. 17, p. 157 (1904). - H. Stichel, Gen. Ins., fasc. 36, p. 13 (1906). - A. phid. pat., Fruhstorfer: A. Seitz, Großschmett. Erde, v. 9, p. 429 (1911). - A. pat., H. Stichel, Therreich, Lief. 34, p. 26 (1912).

A. $p h i d i p p u s$ var., (E. Doubleday \&) Westwood, Gen. Diurn. Lep., v. 2, p. 327 (hab. Java) (1850).

\section{Cohors b. Perakaniformes}

H. Stichel, Gen. Ins., fasc. 36, p. 12 (1906). - Id., Tierreich, Lief. 34 , p. 20 (1912).

perakana Honr. A. phidippus var. perakana, Hon- Malayische rath, Berlin. Ent. Zeit., v. 31, 1887, p. 348 Halbinsel, (part.) (1888). - H. Stichel, Gen. Ins., Java, fasc. 36, p. 12 (1906). - Fruhstorfer: A. Bawean, Seitz, Großschmett. Erde, v. 9, p. 430 (1911). Lombok, - H. Stichel, Tierreich, Lief. 34, p. 21 Borneo, (1912).

nahe der

Torresstraße, ? Java 
subsp. perakana Honr. A. phidippus var. perakana, Honrath, ut antea, t. 6 , f. 2 (1888). - A. perakana, I. Martin, Deutsch. Ent. Zeit., v. 8, p. 260 (1896). - A. phid. pera., Fruhstorfer, Deutsch. Fnt. Zeit. Iep., v. 12, p. 74 (part.) (1899). - A. perakana pera., id., Deutsch. Ent. Zeit. Iris, v. 17, p. 156 (1904). - A. phidippus pera., H. Stichel, Gen. Ins., fasc. 36, p. 12 (part.) (1906). - A. pera. p., Fruhstorfer: A. Seitz, Großschmett. Erde, v. 9, p. 430, t. $105 \mathrm{~b}$ (1911). - A. p. p., H. Stichel, Tierreich, Lief. 34, p. 22 (part.) (1912). - A. phidippus pera., N. D. Riley \& Gabriel, Cat. Type Spec. Rhop. Brit. Mus., pars 1, p. 41 (1924).

? A. $p h$ id ip $p u s$, P. O. T. Snellen, Tijdschr. Ent., v. 20, p. 66 (1877). - F. Moore, Proc. Zool. Soc. London, p. 826 (1878). A. G. Butler, Trans. Linn. Soc. London, ser. 2, v. 1, p. 538 (part.) (1879). - A. p., Distant, Rhop. Malay., p. 538 (1886). - O. Staudinger (\& Schatz), Exot. Schmett., v. 1, p. 187 (1887). - F. Moore, Lep. Ind., v. 2, p. 179 (part, in cit.) (1895).

subsp. $t$ a $e n$ i a Fruhst., A.t., Fruhstorfer, Soc. Java Ent., v. 14, p. 3 (1899). - Id., Deutsch. Ent. Zeit., v. 12 , p. 73 (1899). - A. phiduppus (err. typ.) forma temp. t., id., Deutsch. Ent. Zeit. Iris, v. 17, p. 156 (distr. part.) (1904). - A. phidippus $p$. forma $t$., H. Stichel, Gen. Ins., fasc. 36, p. 10 (1906). - A. p. t., Frulistorfer: A. Seitz, Großschmett. Erde, v. 9, p. 430, t. 105 a (1911). - A. p. t., forma principalis, H. Stichel, Tierreich, Lief. 34, p. 22 (1912). - A. t., Moulton, Journ. Straits Branch Asiat. Soc., Nr. 65, p. 14 (? A. phidippus local form) (1913).

subsp. incisa Fruhst. A. phidippus (?) forma i., Fruhstorfer, Soc. Entom., v. 20, p. 130 ; p. 140 , fig. (1905). - A. p. baweanica forma i., H. Stichel, Gen. Ins., fasc. 36, p. 58 (1906). - A. perakana i., Fruhstorfer: A. Seitz, Großschmett. Erde, v. 9, p. 430 (1911). - A. p. taenia forma i., H. Stichel, Tierreich, Lief. 36, p. 23 (1912). (Subsp. dubia!)

subsp. o rasis Fruhst. A. phidippus lombo- Lombok kiana forma 0. . Frulstorfor, Soc. Entom., v. 20, p. 130 (1905). - H. Stichel, Gen. Ins., fasc. 46 , p. 59 (1906). - A. peratiana o., Fruhstorfer: A. Seitz, Großschmott. Erle, v. 9 , p. 430 (1911). - A. p. taenia forma 0. , H. Stichel, Tierreich, Lief. 34, p. 23 (1912). (Subsp. dub.)

subsp. Staudingeri Röb. A. s., Röber, Südost-

Ent. Nachr, v. 26, p. 202 (1900). - A. Borneo perakana s., Fruhstorfer, Deutsch. Ent. Zeit. Iris, v. 17, p. 156 (1904). - H. Stichel,

Malayische Halbinsel, ? NordostSumatra

\section{Bawean}


Gen. Ins., fasc. 34, p. 23 (1906). - Fruhstorfer: A. Seitz, Großschmett. Erde, v. 9, p. 430 (1911). - H. Stichel, Tierreich, Lief. 34, p. 22, 23 (1912). - Moulton, Journ. Straits Branch Asiat. Soc., Nr. 65, p. 14 (1913).

subsp. natuna Fruhst. A. peraliana n., Natuna Fruhstorfer, Deutsch. Ent. Zeit., v. 17, p. 153, 157 (1904). - Id.: A. Seitz, Großschmett. Erde, v. 9, p. 430 (1911).

A. $p$ er ak a na p., H. Stichel, Gen. Ins., fasc. 36, p. 12 (in cit.) (1906). - Id., Tierreich, Lief. 34, p. 22 (1912).

- virgata Butl. A. v., A. G. Butler, Trans. Ent. Soc. London, p. 486 (part.) (1870). - Fruhstorfer: A. Seitz, Großschmett. Ende, v. 9, p. 431 (1911). - H. Stichel, Tierreich, Lief. 34 , p. 8,23 (1912).

subsp. virgata Butl. A. v., A. G. Butler, Südut antea $(1870)$. W. F. Kirby, Cat. Colebes Diurn. Lep., p. 646 (1871). - O. Staudinger (\& Schatz), Exot. Schmett., v. 1, p. 188 (1887). - Pseudamathusia v., F. Moore, Tep. Ind., v. 2, ए. 182 (1895). - Fruhstorfer, Deutsch. Ent. Zeit. Lep., v. 12, p. 77 (1899). - A. (Pseudam.) v., Röber, Ent. Nachr., v. 26, p. 202 (1900). - Fruhstorfer, Deutsch. Ent. Zeit. Lop., ₹. 17, p. 157 (1904). - Amathusia v., H. Stichel, Gen. Ins., fasc. 36, p. 12, t. 2, f. 1 (1906). - A. v. v., Fruhstorfer: A. Seitz, Großschmett: Erde, v. 9, p. 431 (1911). - H. Stichel, Tierreich, Lief. 34, p. 24 (1912). A. v., N. D. Riley \& Gabriel, Cat. Type Spec. Rhop. Brit. Mus., pars 1, p. 60 (1924).

Ribbei Honr. Pseudamathusia r., Honrath, Corr.-Bl. Ent. Ver. Iris, v. 1, p. 91 (1886). - Amathusia r., E. Haase, l. c., p. 103 (Organ. odor.) (1886). - A. (Pseudamathusia) $r ., 0$. Staudinger (\& Schatz), Exot. Schmett., p. 187, 188 (1887). - E. Haaso, Corr.-Bl. Ent. Ver. 'Iris, v. 1, p. 308 (Organ. odor.) (1888). - Pseudamathusia $r$., Holland, Proc. Boston Soc. Nat. Hist., v. 25, p. $59(1890)$. - W. Rothschild, Deutsch. Ent. Zeit. Lep., v. 5, p. 434 (1892). Amathusia r., Röber, Ent. Nachr., v. 26, p. 202 (Synon.) (1900).

subsp. thoanthea Fruhst. A. virgata $t$., NordFruhstorfer: A. Seitz, Großschmett. Erde, Celebes v. 9, p. 431 (1911). - H. Stichel, Tierreich, Lief. 34, p. 24 (1912).

masina (Fruhst.) Pseudamathusia m., Fruhstorfer, Borneo, Deutsch. Ent. Zeit. Tris, v. 17, p. 155, 157 Bangka (1904). - Amathusia m., id.: A. Seitz, Großschmett. Erde, v. 9, p. 431 (1911). H. Stichel, Tiermeich, Lief. 34, p. 8, 25 (1912). 


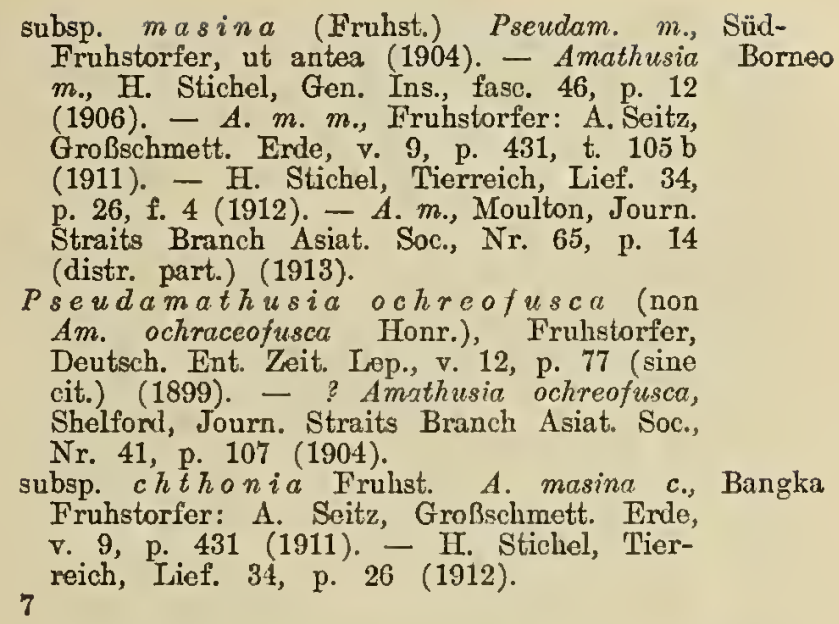

\section{Genus Amafhuxidia O. Stauding.}

A mathuxidia (Subgen.), O. Staudinger (\& Schatz), Exot. Schmett., v. 1, p. 188 (1887). - A., F. Moore, Lep. Ind., v. 2, p. 172, 176 (Typ. A, amythaon) (1895). - L. Martin, Deutsch. Ent. Zeit. Lep., v. 8, 1895, p. 260 (1896). - Nicéville \& L. Martin, Journ. Asiat. Soc. Bengal, v. 64. II, p. 393 (1896). - Fruhstorfer, Berliu. Ent. Zeit., v. 11, 1895, p. 301 (1897). - H. Stichel, Deutsch. Ent. Zeit. Lep., v. 15, p. 59 (nota) (1902). - Röber, Ent. Zeit. Stettin, v. 64, p. 339 (nom. van.) (1903). - Fruhstorfer, Berlin. Ent. Zeit., v. 49, p. 190 (1904). - Shelford, Journ. Straits Branch Asiat. Soc., Nr. 41, p. 107 (1904). - H. Stichel, Gen. Ins., fasc. 36, p. 13, t. 1, f. 2 (Morphol.) (1906). - Fruhstorfer: A. Soitz, Großschmett. Erde, v. 9, p. 431; p. 432 (Artengruppe) (1911). H. Stichel, Tierreicl, Lief. 34, p. 4, 27, f. 5 (Morphol.) (1912). W. H. Evans, Joum. Bombay Nat. Hist. Soc., v. 21, p. 571 (1912). Eecke, Tool. Mededeel. Leiden, v. 4, p. 83 (1918). - W. H. Evans, Journ. Bombay Nat. Hist. Soc., v. 29, p. 793, 796 (sine synon.), t. 16, E 7 (1923). - Moulton, Journ. Straits Branch Asiat. Soc., N1: 65 , p. 14 (1913). - W. H. Evans, Identif. Ind. Butterfl., p. 84, 86 (sine synon.) t. 16, E 7 (1927).

A mathusia (non Fabr.), E. Doubleday, Ann. Mag. Nat. Hist., v. 19, p. 175 (1847). - J. O. Westwood, Cab. Orient. Entom., p. 39 (1848). - E. Doubleday, List Iep. Brit. Mus., App., p. 29 (1848). - A. (Thoraessa Boisd. MS, part.) (E. Doubleday \&) Westwood, Gen. Diurn. Lep., v. 2, p. $326(1851)$. - A. (part.), Clienu \& H. Lucas, Enc. Hist. Nat., Papill., p. 162 (1851). - Horsfiold \& F. Moore, Cat. Lep. Mus. E.-Ind. Comp., v. 1, p. 209 (1857). - HerrichSchäffer, Corr.-Bl. Zool.-Min. Ver. Regensb., v. 19, p. 88 (1865). - A., O. \& R. Felder, Reise Novara, v. 2. II, p. 461 (1866). - A. G. Butler, Ent. Monthly Mag., v. 6, p. 55 (1869). - Id., Lep. Exot., p. 28 (1870). - A., (part.), WV. F. Kirby, Cat. Diurn. Lep., p. 115 (1871). - Id., Scient. Proc. R. Dublin Soc., ser. 2, v. 2, p. 300 (1880). - Marshall \& Nicévillo, Butt. Ind. Burmah Ceyl., v. 1. II, p. 282, 288 (1883). - A., Honrath, Berlin. Ent. Zeit., v. 28, p. 
206 (1884). - A. (part.), Distant, Rhop. Malay., p. 423 (1886). - E. Haase, Corr.-Bl. Ent. Vor. Iris, v. 1, p. 102 (1886). - G. Semper: O. Semper, Reis. Arch. Philippin., pars 2. V, Schmett., p. 70 (1887). - Wood-Mason \& Nicéville, Journ. Asiat. Soc. Bengal, v. 55. -II, 1886, p. 353 (1887). - A., Distant \& Pryer, Ann. Mag. Nat. Hist., ser. 5, v. 19, p. 51 (1887). - E. Haase, Corr.-Bl. Ent. Ver. Iris, v. 1, p. 303 (1888). - Elwes (\& O. Möller), Trans. Ent. Soc. London, p. 282, 334 (1888). - Doherty, Journ. Asiat. Soc. Bengal, v. 60. IT, p. 25 (1891). Swinhoe, Trans. Ent. Soc. London, p. 275 (1893). - Niceville, Gazctteer Sikhim, List Butterfl., p. 130 (1894). A. (part.), Elera, Cat. Faun. Filipinas, v. 2, p. 270 (1895). - A., Eimer (\& Fickert), Entstehg. d. Arten, pars 2, Orthogen., p. 117 etc. (1897). - Fruhstorfer, Berlin. Ent. Zeit., v. 49, p. 189 (1904). - A. (part.), Bingham, Faun. Brit. Ind., Butterfl., v. 1, p. 187 (1905). - A., Tytler, Journ. Bombay Nat. Hist. Soc., v. 21, p. 54 (1911). Eecke, Not. L̇oyden Mus., v. 35, p. 243 (1913). - Id., l. c., จ. 36, p. 227 (1914). - A. (part.), Ollenbach, Journ. Bomb. Nat. Hist. Soc., v. 27, p. 887 (1921). - Antram, Butterfl. Ind., p. 117 (1924). - N. D. Riley \& Gabriel, Cat. Type Spec. Rhop. Brit. Mus,, pars 1, ,p. 7 ete. (1924).

$Z$ e $u x a m a t h u s i a$ (Subgen.), O. Staudinger (\& Sehatz), Exot. Schmett., v. 1, p. 188 (1887). - Z., W. Rothschild; Deutsch. Ent. Zeit., v. 5, p. 434 (1894). - Fruhstorfer, Berlin. Ent. Zeit., v. 43, Sitzgsber. p. (4) (1898). - Id., I. c., v. 44, p. 59 (1899). - Id., Soc. Entoin., v. 13, p. 14 (1899). - H. Stichel, Deutseh. Ent. Zoit. Iris, v. 15, p. 59 (nota) (1902). - Röber, Ent. Zeit. Stettin, v. 64, p. 339 (nom. vanum) (1903). - Z. (Artengruppe), Fruhstorfer: A. Seitz, Großschmett. Ende, v. 9, p. 433 (1911).

amythaon (Doubl.) Amathusia a., E. Doubleday, Ann. Sikkim (?),

Mag. Nat. Hist., v. 19, p. 175 (1847). - Assam, Amathuxidia a., H. Stichel, Gen. Ins., fasc Burma, 36, p. 14 (1906). - Fruhstorfer: A. Seitz, Siam, Großschmett. Erde, v. 9, p. 432 (1911). - Malayische

II. Stichel, Tierreich, Lief. 34, p. 29 (1912). - Godfrey, Journ. Siam Soc. Nat. Hist., v. 7, p. 260 (1930).

Halbinsel,

Sumatra,

Java,

Borneo,

Philippinen

subsp. a mythaon (Doubl.) Amathusia a., E. Ost-Bengalen Doubleday, Ann. Mag. Nat. Hist., v. 19, p. 175 (1847). - J. O. Westwood, Cab. Orient. Entom., p. 39, t. 19, f. $1-3(0$, $)$ (1848). - E. Doubleday, List Lep. Brit. Mus., App., p. 29 (1848). - A. a. (Thoraessa thessaTus Boisduval MIS), (E. Doubleday \&) Westwood, Gen. Diurn. Lep., v. 2, p. 327 (1851).

(Sylhet),

Assam,

Burma,

Tenasserim,

Nond-Siam,

P Sikkim - A. a., Chenu \& H. Lucas, Enc. Hist. Nat., Papill., p. 162 (1851). - Horsfield \& F. Moore, Cat. Lop. Mus. E.-Ind. Comp., v. 1, p. 260 (1857). - Herrich-Schäffer, Corr.B1. Zool.-Min. Ver. Regensb., v. 19, p. 88 (1865). - W. F. Kirby, Cat. Diurn. Lep., p. 115 (1871). - G. F. L. Marshall \& Niceville, Butt. Ind. Burmah, Ceyl., v. 1. II, p. 
291 (1883). - A. (Amathuxidia) a., O. Staudinger (\& Schatz), Exot. Scbmett., v. 1, p. 188 (1887). - Amathuxidia a., F. Moore, Lep. Ind., จ. 2, p. 176, t. 145, f. 1, 1a $\left(\sigma^{\top}, q\right)$ (1895). - Shelford, Journ. Asiat. Soc. Bengal, v. 41. II, p. 107 (1904). - A. a. a., Fruhstorfor, Berlin. Ent. Zeit., v. 49, p. 190 (1904). - Amathusia a., Bingbam, Fauna Brit. Ind., Iop., จ. 1, p. 188 (1905). Amathuxidia a. a., H. Stichel, Gen. Ins., fasc. 36, p. 14 (1906). - Fruhstorfer: A. Seitz, Großschmett. Erde, v. 9, p. 432 (1911) Amathusia a., Tytler, Journ. Bombay Nat. Hist. Soc., v. 21, p. 54 (1911). - Amathuxidia a. a., H. Stichel, Tiermeich, Lief. 34, p. 30, 31 (1912). - Amathusia (Amathuxidia) a., W. H. Evans, Journ. Bombay Nat. Hist. Soc., v. 21, p. 571 (1912). - Amathresia a., Ollenbacb, Journ. Bombay Nat. Hist. Soc., v. 27, p. 887 (1921). - Amathuxidia $a .$, W. H. Evans, 1. c., v. 29, p. 796, t. 16, f. E 8. 1 (1923). - Amathusia a., N. D. Riley, Cat. Type Spec. Rhop. Brit. Mus., pars 1, p. 7 (1924). - Antram, Butterfl. Ind., p. 117, f. 243,244 (1924). - Amathuxidia a., W. H. Evans, Identif. Ind. Butterfl., p. 86, t. 16, $f$. E 8. 1 (1927). - A. a. a., Godfrey, Journ. Siam Soc. Nat. Hist. Suppl., v. 7, p. 122 (1927). - Id., 1. c., p. 260 (1930).

portheus (C. \& R. Feld.) Amathusia p., C. \& R. Feldor, Reise. Novara, v. 2. II, p. 461 (patria falsa: Java) (1866). - A. G. Butler, Ent. Monthly Mag., v. 6, p. 55 (1869). - W. F. Kirby, Cat. Diurn. Lep., p. 115 (1871). - G. F. L. Marshall \& Nicéville, Butt. Ind. Burm. Ceylon, v. 1, p. 293, fig. (1883). - Wood-Mason \& Nicéville, Journ. Asiat. Soc. Bengal, จ. 55. II, p. 353 (1887). - Elwes (\& O. Möller), Trans. Ent. Soc. Londoṇ, p. 275 (1888). - A. portheos (!), Swinboe, Trans. Ent. Soc. London, p. 275 (1893). - Amathusia portheus, Nicéville, Gazetteer Sikhim, List Butterfl., p. 130 (1894).

Westwoodi (Butl.) Amathusia w., A. G. Butler, Ent. Monthly Mag., v. 6, p. 55 (1869). - TV. F. Kirby, Scient. Proc. R. Dublin Soc., ser. 2, v. 2, p. 300 (1880). G. F. I. Marshall \& Nicéville, Butt. Ind. Burmah, Ceyl., v. 1. II, p. 292 (1883).

subsp. dilucida (Honr.) Amathusia d., Malayiscbe Honratb, Berlin. Eut. Zeit., v. 28, p. 206, t. Halbinsel 5 , f. $3 \mathrm{a}\left(\sigma^{7}\right)$, t. 4 , f. $3 \mathrm{~b}$ (Q) $(1884)$. - Distant, Rlop. Malay., p. 423, t. 38, f. 7 (1886). - E. Haase, Corr.-Bl. Ent. Ver. Iris, v. 1, p. 102,309 (Org. odor.) (1886 \& 88). Amathusia (Amathuxidia) d., O. Staudinger (\& Scbatz), Exot. Schmett., v. 1, p. 188 (1887). - Amathusia d., Elwes (\& O. Möller), Trans. Ent. Soc. London, p. 282 (1888). 
- Amathuxidia d., F. Moone, Lep. Ind., v. 2, p. 178 (1895). - Amathusia d., Eimer (\& Fickert), Entstehg. d. Arton, pars 2, Orthgen., p. 117 , f. 135 ; p. 189,235 , f. 189 (1897). - Amathuxidia amythaon d., H. Stichel, Gen. Ins., fasc. 36, p. 15 (1906). - Fruhstorfer: A. Seitr, Großschmett. Erde, v. 9, p. 432 (1911). - H. Stichel, Tierreich, Lief. 34, p. 31, 32 (1912). - Amathusia d., N. D. Riley, Cat. Type Spec. Rhop. Brit. Mus., pars 1, p. 17 (1924). - Amathuxidia amythaon d., Godfrey, Journ. Siam Soc. Nat. Hist. Suppl., v. 7, p. 260 (1930).

A math $u$ s ia a $m$ yth a on, Elwes, Proc. Zool. Soc. London, p. 270 (1891).

subsp. $l u c$ id a Fruhst. A. amythaon l., Fruh- Sumatra storfer, Berlin. Ent. Zeit., v. 49, p. 189 (1904). - H. Stichel, Gen. Ins., fasc. 36, p. 15 (1906). - Fruhstorfer: A. Seitz, Großschmett. Erde, v. 9, p. 432 (1911). H. Stichel, Tierreich, Lief. 34, p. .31, 32 (1912). - Amathusia (amythaon) l., Eecke, Not. Toyden Mus., v. 36, p. 227 (1914).

A mathuxidia dilucida, I. Martin, Deutsch. Ent. Zeit., v. 8, p. 260 (1895). Nicéville \& L. Martin, Journ. Asiat. Soc. Bengal, v. 64. II, 1895 , p. 393 (1896).

subsp. insularis (Doherty) Amathusia Engano, amythaon var. $i$. , Doherty, Journ. Asiat. Soc. Nias Bengal, v. 60 . II, p. 25 (1891). - Amathuxidia i., F. Moore, Lep. Ind., v. 2, p. 178 (1895). - Amathusia amythaon i., Frulistorfer, Berlin. Ent. Zeit., v. 49, p. 189 (1904). - Amathuxidia a. i., H. Stichel, Gen. Ins., fasc. 36, p. 15 (1906). - Fruhstorfer: A. Seitz, Großsschmett. Erde, v. 9, p. 432 (1911). - H. Stichel, Tierreich, Lief. 34 , p. 31,32 (1912). - Amathusia a. "Lokalrasse" $i$, Eecke, Not. Leyden Mus., v. 36, p. 227 (1914).

subsp. coerulilata Eecke A. a. c., Eecke,

Not. Leyden Mius., v. 36, p. 227 (1914). -

Id., Zool. Mededeel. Leiden, v. 4, p. 83, t. 8, f. 3 (1918).

A. amytha on, Eecke, Not. Leyden Mus., v. 35, p. 243 (1913).

- subsp. pylaon (C. \& R. Feld.) Amathusia ṕ, Java

C. \& R. Telder, Reise Novara, r. 2. II, p. 461 (1866). - W. F. Kirby, Cat. Diurn. Lep., p. 115 (1871). - A. (Amathuxidia) amythaon var. (?) p., O. Staudinger (\& Schatz), Exot. Schmett., v. 1, p. 188 (1887). - Amathuxidia p., F. Moore, Lep. Ind., v. 2, p. 178 (1895). - A. amythaon p., Fruhstorfer, Berlin. Ent. Zeit., v. 49, p. 190 (1904). - H. Stichel, Gen. Ins., fase. 36, p. 15 (1906). - Frulistorfer: A. Scitz, Großschmett. Erde, v. 9, p. 432 (1911). - H. Stichel, Tierreich, Lief. 34, p. 31, 33 (1912).

Pulu Babi, Pulu Lasia (westl. Sumatra) 
porthaon (C. \& R. Feld.) Amathusia p., C. \& R. Felder, Reise Novara, v. 2. II, p. 461 (1866). - W. F. Kirby, Cat. Diurn. Lep., p. 115 (1871). - G. F. L. Marshall \& Nicéville, Butt. Ind. Burm. Ceylon, จ. 1. II, p. 293 (1883). - A. (Amathuxidia) amythaon var. (?) p., O. Staudinger (\& Schatz), Exot. Schmett., v. 1, p. 188 (1887). - Amathuxidia p., F. Moore, Lop. Ind., v. 2, p. 178 (1895). Fruhstorfer, Berlin. Ent. Zoit., v. 41,1896 , p. 301 (1897). - Amathusia (amythaon) porthaon, Eecke, Not. Leyden Mus., v. 36, p. 227 (1914).

subsp. ottomana (Butl.) Amathusia o., A. Nord-Borneo G. Butler, Ent. Monthly Mag., v. 6, p. 55 (1869). - Id., Lop. Exot., p. 28, t. 11, f. 1 (1870). - W. F. Kirby, Cat. Diurn. Lep., p. 115 (1871). - Herb. Druce, Proc. Zool. Soc., Iond., p. 341 (1873). - A. (Amathuxidia) amythaon var. 0., O. Staudinger (\& Schatz), Exot. Sehmett., v. 1, p. 188 (1887). - Amathusia o., Distant \& Pryer, Ann. Mag. Nat. Hist., ser. 5, v. 19, p. 51 (1887). - E. Haase, Corr.-Bl. Ent. Ver. Iris, v. 1, p. 309 (Organ. odor.) (1888). - Amathuxidia o., F. Moore, Lep. Ind., v. 2, p. 178 (1895). - A. dilucida forma o., L. Martin, Deutsch. Ent. Zeit. Lep., จ. 8, p. 260 (1895). - A. amythaon 0., Shelford, Journ. Straits Branch Asiat. Soc., Nr. 41, p. 107 (1904). - Fruhstorfer, Berlin. Ent. Zoit., v. 49, p. 190 (1904). - H. Stichel, Gen. Ins., fasc. 36, p. 15 (1906). Fruhstorfer: A. Seitz, Großschmott. Ende, V. 9, p. 432, t. $102 \mathrm{a}\left(\sigma^{*}\right.$, Q) (1911). - H. Stichel, Ticrreich, Lief. 34, p. 31, 33 (1912). - Moulton, Journ. Straits Branch Asiat. Soc., Nr. 65, p. 14 (1913). - Amathusia o., Eecke, Not. Leyden Mus., v. 36, p. 227 (1914). - N. D. Riley, Cat. Type Spec. Rhop. Brit. Mus., pars 1, p. 42 (1924).

subsp. o c $t a^{\circ}$ c $i l$ ia Fruhst. A. a. o., Fruhstorfer: A. Seitz, Großschmett. Frde, v. 9, p. 433 (1911) - H. Stichol, Tierreich, Lief. 34, p. 31, 34 (1912).

subsp. $p h i l i p p i n a$ Moore A. p., F. Moore, Panaon Lep. Ind., v. 2, p. 178 (1895). - A. amy- (Philippinen) thaon p., Fruhstorfer, Berlin. Ent. Zeit., v. 49 , p. $190(1904)$ - 'H. Stichel, Gen. Ins., fasc. 36, p. 15 (1906). - Fruhstorfer: A. Seitz, Großschmett. Erde, v. 9, p. 433 (1911). - H. Stichel, Tierreich, Lief. 34, p. 31,34 (1912).

A mathusia porthaon (?), G. Semper: C. Semper, Reis. Arch. Philippin., pars 2. V, Schmett., v. 1, p. 71 , t. 13 , f. 7 (Q); p. $332\left(\sigma^{7}\right)(1887 \& 92)$.

subsp. perinthas Fruhst. A. a. p., Fruh- Mindanao storfer: A. Seitz, Großschmett. Erde, v. 9, 
p. 433 (1911). - H. Stichel, Tierreich, Lief. 34, p. 31, 35 (1912).

Amathusia porthaon (non Felder), Elera, Cat. Fauna Filippin., v. 2, p. 270 (sine cit.) (1895).

- Plateni (Stauding.) Amathusia (Zeuxamathusia) p., O. Staudinger (\& Schatz), Exot. Schmett., v. 1 , p. 188 (1887). - Amathuxidia p., H. Stichel, Gen. Ins., fasc. 36, p. 15 (1906). Fruhstorfer: A. Seitz, Großschmett. Ende, v. 9, p. 433 (1911). - H. Stichel, Tierneich, Lief. 34 , p. 35 (1912).

subsp. Platen $i$ (Stauding.) Amathusia (Zeuxamathusia) p., $\mathrm{O}$. Staudinger, ut antea (part.) (1887). - E. Haasc, Corr.-Bl. Ent. Ver. Iris, v. 1, p. 309 (Organ. odor.) (1888). - Z. p., W. Rothschild, Deutsch. Ent. Zeit. Lep., p. 434 (1892). - Fruhstorfer, Berlin. Ent. Zeit., v. 43, Sitz.-Ber., p. (4) (not. biol.) (1898). - Amathrxidia p., id., Berlin. Ent. Zeit., v. 49, p. 190 (1901). - A. p. p., H. Stichel, Gen. Ins., fasc. 36 , p. 15, t. 2 , f. $2\left(0^{x}\right)$; t. 3 , f. 3 (Q) (1906). - Frulustorfer: A. Seitz, Großschmett. Erde, v. 9, p. 433 (1911). - H. Stichel, Tierreich, Lief. 34, p. 29, 36 (1912). - A. p., M. Hering, Biol. Schmett., p. 154 (odor) (1926).

subsp. suprema (Fruhst.) Zeuxamathusia Sula Mangoli plateni s., Fruhstorfer, Soc. Entom., v. 13, p. 161 (1899). - Id., Berlin. Ent. Zeit., v. 44 , p. 59 (1899). - Amathuxidia p. s., id., Berlin. Ent. Zeit., v. 49, p. 190 (1904). - H. Stichel, Gen. Ins., fasc. 36, p. 15 (1906). - Fruhstorfer: A. Seitz, Großschmett. Erde, v. 9, p. 433, t. 102 b (ㅇ) (1911). - H. Stichel, Tierreich, Lief. 34, p. 2 36 (1912).

\section{Celebes,}

Sula-Inseln

\section{Celebes}

\section{Genus Thaumantis Jac. Hübner}

Jac. Hübner, Samml. Exot. Schmett., v. 2, Index, p. 2, t. [61] (1820-26]. - Boisduval, Spec. Gén. Lép., v. 1, t. 12 (8 B) (1836). - E. Blanchard, Hist. An. Artic., v. 3, p. 451 (1840). - Id., Hist. Ins., v. 2, p. 335 (1845). - Thaumantis (part.), (E. Doubleday) \&) Westwood, Gen. Diurn. Lop., v. 2, p. 335 (1851). - Chenu \& H. Lucas, Enc. Hist. Nat., Papill., p. 166 (1851). - T., Mén6trís, Enum. Corp. Anim. Mus. Petropol., Lep., pars 1, p. 37 (1855). T. (part.), Horsfield \& F. Moore, Cat. Lep. Mus. E.-Ind. Comp., v. 1, p. 214 (1857). - J. O. Westwoor, Trans. Ent. Soc. London, n. ser., v. 4, p. 170 (1858). - T., Carus-Gerstäcker, Handb. Zool., v. 2, p. 232 (1863). - T. (part.), Herrich-Schäffer, Corr,-Bl. Zool.Min. Ver. Regensb., v. 18 , p. 112; v. 19, p. 88 (1864 \& 65). T., H. W. Bates, Journ. Entom., v. 2, p. 177 (1866). - T. (parti) W. F. Kirby, Cat. Diurn. Lep., p. 119 (1871). - T., S. H. Scudder, Proc. Amer. Ac. Sci., v. 10, p. 279 (Typ. T. odana) (1875). E. Martens, Preuß. Exped. Ost-Asien, v. 1, p. 290 (1875). - Wallace, Geogr. Distrib. Anim., v. 1, p. 319 (1876). - F. Müller, Jena. Zeitschr. Naturw., v. 11, p. 102 (Physiol.) (1877). - Id., Kosmos, 
Leipzig, v. 1, p. 394 (1877). - Thaumantias (!), (part.), W. F. Kirby, Entomologist, v. 10, p. 291, 292 (1877). - Thaumantis (part.) A. G. Butler, Trans. Linn. Soc. London, ser. 2, Zool., v. 1, p. 538 (1879). - W. F. Kirby, Scient. Proc. K. Dublin Soc., ser. 2, v. 2, p. 300 (1880). - C. Orüger (sec. Kirby), Berlin. Ent. Zeit., v. 25, p. 111 (1881). - Distant, Rhop. Malay., p. 69, 77, 426 (1882 \& 86). - G. F. L. Marshall \& Nicéville, Butt. Ind. Burm. Ceyl., v. 1, p. 304 (1883). - Thaumanthis, B. Gerhard, Berlin. Ent. Zeit., v. 27, p. 181 (distr. geogr.) (1883). - Thaumantis (part.), H. Haase, Corr.-Bl. Ent. Ver. Iris, v. 1, p. $10 \frac{4}{2}$ (Organ. odor.) (1886). T., Dolierty, Journ. Asiat. Soc. Bengal, v. 55. II, p. 109 (1886). L. Glaser, Cat. Etymol. Col. Lep., p. 314 (spec. omiss.) (1887). T. (part.), E. Haase, Corr.-Bl. Intom. Ver. Iris, v. 1, p. 306 (1888). - O. Staudingor (\& Schatz), Exot. Schmett., v. 1, p. 203 (1887). - Röber, 1. c., v. 2, t. 30 (Morphol.), p. 184, 185 (1888 \& 89). - T., Skertchly, Ann. Mag. Nat. Hist., ser. 6, v. 3, p. 210, 211 (not. biol.) (1889). - B. Hagen, Tijdschr. Nederl. Aardr. Gen., ser. 2, v. 7, p. 200 (1890). - T., Poujade, Nouv. Arch. Mus. Hist. Nat. Paris, ser. 3, v. 3, p. 264 (1891). - Swinhoe, Trans. Ent. Soc. Iondon, p. 276 (1893). - Thaumantias (!), W. F. Kirby, Handb. Lep. (Allens. Nat. Libr.), v. 1, p. 197, 198 (1894). - Thaumantis, F. Moore, Lep. Ind., v. 2, p. 184 (Typ. T. odana) (1895). - L. Martin, Deutsch. Ent. Zeit. Lep., v. 8, 1895, p. 256 (1896). - D. Reuter, Leta Soc. Sci. Fenn., v. 22, p. 108 (1896). - T. (part.), Eimer. (\& Fickert), Entstehg. d. Arten, pars 2, Orthogen., p. 155, 178 (1897). - T., A. Pagenstecher, Abl. Senckenb. Naturf. Ges., v. 23, p. 406 (1897). - W. F. Kirby: Hübner \& Geyer, Samml. Exot. Schmett., Neue Ausg., v. 3, p. 118 (1901). - Fruhstorfer, Deutsch. Ent. Zeit. Iris, v. 15, p. 177 (1902). - H. Stichel, l. e., v. 15, p' 59 (1902). - Röber, Ent. Zoit. Stettin, v. 64, p. 338 (1903). L. C. H. Young, Journ. Bombay Nat. Hist. Soc., v. 15, 1903, p. 298, 485 (1903 \& 04). - T., Sectio 1, Shelford, Journ. Straits Branch Asiat. Soc., Nr. 41, p. 108 (1904). - T., Bingham, Faun. Brit. Ind., Butterfl., v. 1, p. 182,189 (1905). - H. Stichel, Gen. Ins., fase. 36, p. 15, t. 1, f. 3 (Morphol. fig. vitiosa) (1906). - A. Pagenstecher, Geogr. Verbr. Schmett., p. 412 (1909). - Bell, Journ. Bombay Nat. Hist. Soc., v. 19, p. 469 (1909). - L. Martin, Mitt. Münch. Ent. Ges., p. 70 (1910). - T. (Kringana), Strand, Deutsch. Ent. Zeit. Iris, v. 24, p. 193 (1910). - T., Fruhstorfer: A. Seitz, Großschmett. Erde, v. 9, p. 437 (1911). - H. Stichel, Tierreich, Lief. 34, p. 4; 37, f. 6 (Morphol.) (1912). - W. H. Evans, Journ. Bombay Nat. Hist. Soc., v. 21, p. 571 (1912). - Moulton, Journ. Straits Branch Asiat. Sos., Nr. 65, p. 15 (1913). - Eecke, Zool. Mededeel. Loiden, v. 4, p. 84 (1918). - W. H. Evans, Jouru. Bombay Nat. Hist. Sos., v. 29, p. 793, 795, t. 16, E5 (1923). - Antram, Butt. Ind., p. 118 (1921). - Thaumantes (!), M. Hering, Biol. Schmett., p. 154 (1926). - Thaumantis, W. H. Evans, Identif. Ind. Butterfl., p. 84, 86, t. 16, E5 (1927). - A. Seitz, Int. Pundschau, v. 45, p. $24(1928)$.

M o $\operatorname{rpho}$ (part.), Latreille, Enc. Méth., v. 9, p. 11 (1819). - J. B. Godart, l. c., p. 435 (1924). - M., Horsfield, Descr. Cat. Lep. Mus. E.-Ind. Comp., t. 6 (1829). Zinken-Sommer, Nova Acta Ac. Leop., v. 15, p. 165 (1831) - N. (part.), J. O. Westwood, Truns. Ent. Soc. London, п. ser., v. 4, p. 172 (1858). - M. (Thaumantis), Marshall \& Nicéville, Butt. Ind. Burm. Ceyl., p. 306 (1883).

$N$ and o gea, F. Moore, Lep. Ind., v. 2, p. 173, 182 (Typ. $N$. diores) (1895). - Nandoges (!) Orowley, Proc. Zool. Soc. London, p. 505 (1900). - Nandogea, H. Stichel, Deutsch. 
Ent. Zeit. Iris, v. 15, p. 59 (nota) (1902). - R. South, Journ. Bombay Nat. Hist. Soc., v. 22, p. 352 (1913).

$K$ ringana, F. Moore, Lep. Ind., v. 2, p. 185 (Typ. $R$. noureddin) (1895). - Nicéville \& L. Martin, Journ. Asiat. Soc. Bengal, v. 64. II, 1895, p. 395 (1896). - K. (Sectio), Shelford, Journ. Straits Branch Asiat. Soc., Nr. 41, p. 108 (1904). - Thaumantis (R.), Strand, Deutsch. Ent. Zeit. Iris, v. 24, p. 193 (1910).

\section{Sectio Glaucosfillbi}

H. Stichel, Gen. Ins., fasc. 36, p. 17 (1906); Tierreich, Lief. 34, p. 39 (1912).

diores Doubl. T. d., E. Doubleday, Ann. Mag. Nat. SikkimHist., v. 16, p. 234 (part.) (1845). - F. Burma, Stichel, Gen. Ins., fasc. 36, p. 17, 59 (1906). Süd-Tibet, - Fruhstorfer: A. Seitz, Großschmett. Erde, Siamv. 9, p. 438 (1899). - H. Stichel, Tier- Perak, reich, Lief. 34, p. 39, 40 (1912). Hainan

- subsp. di o r es Doubl. T. d., E. Doubleday, ut Sikkim, antea (1845). - (E. Doubleday \&) West- Südostwood, Gen. Diurn. Lep., v. 2, p. 337 (1851). Tibet, - Horsfield \& F. Moore, Cat. Lep. Mus. E.- Siam, Ind. Comp., v. 1, p. 215 (1857). - West- Burma, wood, Trans. Ent. Soc. London, n. ser., v. 4, Tenasserim, p. 171 (1858). - Herrich-Schäffer, Corr.- Perak Bl. Zool.-Min. Ver. Regensb., v. 19, p. 88 (1865). - F. Moore, Proc. Zool. Soc. London, p. 761 (1865). - W. F. Kirby, Cat. Diurn. Lep., p. 120 (1871). - Id., Entomologist, v. 10, p. 292 (1877). - Id., Scient. Proc. R. Dublin Soc., ser. 2, r. 2, p. 300 (1880). - Nicéville, Jourı. Asiat. Soc. Bengal, v. 50. II, p. 57 (1881). - G. F. L. Marshall \& Nicéville, Butt. Ind. Burm. Ceyl., v. 1, p. 304 (1883). - A. G. Butler, Ann. Mag. Nat. Hist., ser. 5, v. 16, p. 302 (1885). - E. Haase, Corr.-B1. Ent. Ver. Iris, v. 1, p. 104 (Organ. odor.) (1886). - Wood-Mason \& Nicéville, Journ. Asiat. Soc. Bengal, v. 55. II, p. 353 (1887), - O. Staudinger (\& Schatz), Exot. Schmett., v. 1, p. 203 (1887). - Elwes (\& O. Möller), Trans. Ent. Soc. London, p. 282, 333 (1888). - E. Haase, Corr.-Bl. Ent. Ver. Iris, v. 1, p. 307 (1888). - Elwes, Proc. Zool. Sac. London, p. 271 (1891). - Poujade, Nouv. Arch. Mus. Hist. Nat. Paris, ser. 3, v. 3, p. 264 (1891). Swinhoe, Trans. Ent. Soc. London, p. 276 (1893). - Nicéville, Gazetteer Sikhim, List Butterfl., p. 131 (1894). - Nandogea d., F. Moore, Iep. Ind., v. 2, p. 182, t. 148, f. 1, $1 \mathrm{a}, \mathrm{b}\left(\mathrm{O}^{2}\right.$, O) $(1895)$. - Thoumantis $d$., Manders, Journ. Bombay Nat. Hist. Soc., v. 16, p. 82 (1904). - Bingham, Fuun. Brit. Ind., Lep., v. 1, p. 190 (1905). - T. d. d., II. Stichel, Gen. Ins., fasc. 36, p. 17,59 (1906). - Fruhstorfer: A. Seitz, Großschmett. Erde, v. 9, p. 438 (non t. 104 a: $=$ 
ramdeo) (1911). - H. Stichel, Tierreich, Lief. 34 , p. 40 (1912). - T. d., W. H. Evans, Journ. Bombay Nat. Hist. Soc., v. 21, p. 571 (1912). - Nandogea d., R. South, l. c., v. 22, p. 352 (1913). - Thaumantis d., W. H. Evans, Journ. Bombay Nat. Hist. Soc., v. 23, p. 538 (1915). - T. d. d., Godfrey, Journ. Nat. Hist. Soc. Siam, v. 2, p. 124 (1916). - T. d., Ollenbach, Journ. Bombay Nat. Fist. Soc., v. 27, p. 887 (1921). - W. H. Evans, 1. c., v. 29, p. 795 (sine synon.), t. 16, f. E 5. 1 (1923). - Antram, Butterfl. Ind., p. 118, f. 245 (1924). - Thaumantes d., M. Hering, Biol. Schmett., p. 154 (odor) (1926). - Thaumantis d., W. H. Evans, Identif. Ind. Butt., p. 86 (sine synon.), t. 16, f. E 5. 1 (1927). - T. d. d., Godfrey, Journ. Siam Sac. Nat. Hist. Suppl., v. 7, p. 121, 261 (1927, 1930). - Rosa, Entomologist, v. 62 , p. $226(1929)$.

forma $r$ a mdeo F. Moore T. $r$., (Horsfield \&)

F. Moore, Cat. Lep. Mus. E.-Ind. Comp., v. Typ.:

1, p. 215 (1857). - Westwood, Trans. Ent. Soc. London, p. 172 (1858). - HerrichSchäffer, Corr.-Bl. Zool.-Min. Ver. Regensb., v. 19, p. 88 (1865). - W. F. Kirby, Cat. Diurn. Lep., p. 120 (1871). - G. F. L. Marshall \& Nicéville, Butt. Ind. Burm. Ceyl., v. 1 , p. 305 , fig. $(1883)$. $-T$. $d$. var. r., 0 . Staudinger (\& Schatz), Exot. Schmett., v. 1, p. 203 (1887). - T. r., E. Haase, Corr.-Bl. Ent. Ver. Iris, v. 1, p. 307 (1888). - Nandogea $d$. "form" $r$., Tr. Moore, Lep. Ind., v. 2, p. 184 (1893). - Thaumantis d. "form" r., E. Y. Watson, Journ. Bombay Nat. Hist. Soc., v. 10, p. $652(1897) .-T . r$., L, C. H. Young, l. c., v. 15, p. 485 (1904). - T. $d$. d. forma $r$, $H$. Stichel, Gen. Ins., fasc. 36, p. 17 (1906). - T. d. r., Truhstorfer: A. Seitz, Großschmett. Erde, v. 9, p. 438 (Zoitform), t. 104 a (diores corr. p. 1134: ramdeo) (1911 \& 27). - T. d. d. forma r., H. Stichel, Tierreich, Lief. 34 , p. 41 (1912). subsp. hainana (Orowley) Nandogea h., Hainan Crowley, Proc. Zool. Soc. London, p. 505 (1900). - T. d. h., H. Stichel, Gen. Ins., fasc. 36, p. 18 (1906). - Fruhstorfer: A. Seitz, Großschmett. Erde, v. 9, p. 438 (1911). - H. Stichel, Tiorreich, Lief. 34 , p. 40, 41 (1912). - Joicey \& Talbot, Bull. Hill Mus., v. 2, p. 184 (1928).

klugius (Zink.-Somm.) Morpho k., Zinken-Sommer, Java, Nov. Acta Ac. Leop., v. 15, p. 165 (part.) Borneo, (1831).

Tha umantis lucipor, H. Stichel, Gen. Ins., fasc. 36, p. 357 (1906). - Fruhstorfer: A. Seitz, Großschmett. Erde, v. 9, p. 438 (1911). - H. Stichel, Tierreich, Lief. 34, p. 41 (1912).

Sumatra, Bangka, Billiton, Malayische Halbinsel 
subsp. klugiu\& (Zink.-Somm.) Morpho k., Java Zinken-Sommer, Nov. Acta Ac. Leop., v. 15, p. $165\left(\sigma^{x}\right.$, non $\left.Q\right)$, t. 15 , f. 11 ( $\left.\sigma^{x}\right)$ (non f. 12, 13: = T. odona o. o) (1831). - Thanmantis K., (E. Doubleday \&) Westwood, Gen. Diurn. Lep., v. 2, p. 337 (1851). Morpho k., id., Trans. Ent. Soc. London, n. ser., v. 4, p. 172 (1858). - Thaumantis k., Herrich-Schäffer, Corr:-Bl. Zool.-Min. Ver. Regensb., v. 19, p. 88 (1865). - IV. F. Kirby, Cat. Diurn. Lep., p. 120 (1871). Kringana k., F. Moore, Lep. Ind., v. 2, p. 185 (1895).

T. l u c i p or, O. Staudinger (\& Schatz), Exot. Schmett., v. 1, p. 20 (part.) (1887). - T. l., T. l. l., H. Stichel, Gen. Ins., fasc. 36, p. 18,59 (1906). - T. l. l., id., Tierreich, Lief. 34, p. 39,43 (part.) (1912).

- subsp. lucipor Westw. T. l., (E. Doubleday Borneo

\&) Westrood, Gen. Diurn. Lep., v. 2, p. 357 (1851). - Horsfield \& F. Moore, Cat. Lep. Mus. E.-Ind. Comp., v. 1, p. 341 (1857). - Westwood, Trans. Ent. Soc. London, n. ser., v. 4 , p. 173, t. 19, f. 1,1 a $\left(0^{7}\right)$, f. 2, 2 a (O) (1858). - Herrich-Schäffer, Corr.B1. Zool.-Min. Ver. Regensb., v. 19, p. 88 (1865). - W. F. Kirby, Cat. Diurn. Lep., p. 120 (1871). - Herb. Druce, Proc. Zool. Soc. London, p. 2 (1873). - W. F. Kirby, Sci. Proc. R. Dublin Soc., ser. 2, v. 2, p. 300 (1880). - G. F. L. Mrarshall \& Nicéville, Butt. Ind. Burm. Ceyl., v. 1. II, p. 306 (1883). - O. Staudinger (\& Schatz), Exot. Schmett., v. 1, p. 203 (part.) (1887). Distant \& Pryer, Ann. Mag. Nat. Hist., ser. 5, v. 19, p. 51 (1887). - E. Haase, Corr.Bl. Ent. Ver. Iris, v. 1, p. 307 (Organ. odor.) (1888). - Kringana l., F. Moore, Lop. Ind., v. 2, p. 185 (part.) (1895). - Thaumantis l., E. Reuter, Acta Soc. Sci. Fenn., v. 22, p. 108 (1896). - Shelford, Journ. Straits Branch Asiat. Soc., Nr. 41, p. 108 (distr. part.) (1904). - Bingham, Faun. Brit. Ind., Lep., v. 1, p. 190 (part.) (1905). - T. l., T. l. l., H. Stichel, Gen. Ins.; fasc. 36, p. 18, 59 (1906). - L. Martin, Mitt. Münch. Ent. Ges., v. 1, p. 72 (Biol.), p. 75, fig. (chrysal.) (1910). - T. l. l., Fruhstorfer: A. Seitz, Großschmett. Erde, †. 9 , p. 438 , t. 104 b (1911). - H. Stichel, Tierreich, Lief. 34, p. 43 (1912). - T. l., Moulton, Journ. Straits Branch. Asiat. Soc,, Nr. 65, p. 15 (1913). - N. D. Riley \& Gabriel, Cat. Type Spec. Rhop. Brit. Mus., pars 1, p. 30 (1924).

subsp. candi a $_{a}$ Fruhst. T. lucipor c., Fruh- Sumatra, storfer, Soc. Entom., v. 20, p. 113 (1905). Bangka, - H. Stichel, Gen. Ins., fasc. 36, p. 59 Billiton, (1906). - Fruhstorfer: A. Seitz, Groß- Malayische 
schmett. Erde, v. 9, p. 439; 1111, t. 104 b

Halbinsel, (non $101 \mathrm{~b}$ ) (candica, corr. p. 1135: candika) (1921 \& 27). - T. l. candika, H. Stichel, Tierreich, Lief. 34, p. 43, 44 (1912).

T. klugius (non Morpho k. Zink.-Somm.), Weyenbergh, Pet. Nouv. Ent., Année 6, p. 404 (1874). - P. C. T. Snellen, Tijdschr. Ent., v. 33, p. 290 (1890). - Id.s Not. Leyden Museum, v. 13, p. 136 (1891).

T. lucipor (non Westwood), Distant, Rhop. Malay., p. 77 (part.), t. 9, f. 8, 9 ( $\sigma^{n}, 0$ ) (1882). - Elwes (\& O Möller), Trans. Ent. Soc. London, p. 282 (1888). - B. Hagen, Tijdschr. Nederl. Aardı. Gen., ser. 2, v. 7, p. 200 (1890). - L. Martin, Berlin. Ent. Zeit., v. 35, p. 6 (1890). - Elwes, Proc. Zool. Soc. London, p. 271 (1891). Kringana $l$., F. Moore, Lep. Ind., v. 2, p. 185 (in cit.) (1895). - T. (Kringana) l., Nicéville \& L. Martin, Jouru. Asiat. Soe. Bengal, v. 64. II, 1895, p. 395 (1896). T. l., L. Martin, Deutsch. Ent. Zeit. Lep., v. 8, p. 256 (1896). - Bingham, Faun. Brit. Ind., Lep., v. 1, p. 190 (part.) (1905). - T. (Kringana) l., Strand, Deutsch. Ent. Zeit. Iris, v. 24, p. $193(1910)$. - T. l., W. H. Evans, Journ. Bombay Nat. Hist. Soc., v. 21, p. 571 (1912). - Ollenbach, 1. c., v. 27, p. 887 (1921). - T. l. l., Pendlebury, Journ. Feder. Malay. Stat. Mins., v. 11, p. 34 (1923). - T. l., W. H. Evans, Journ. Bombay Nat. Hist. Soc., v. 29, p. 795 (1923). Id., Identif. Ind. Butterfl., p. 86 (1927). Godfrey, Journ. Siam Soc. Nat. Hist., Suppl, v. 7, p. 261 (distr. part.) (1930).

- odana (God.) Morpho o., (Latreille \&) J. B. Godart, Fnc. Méth., v. 9 , p. 445 (1824). 36, p. 18, 59 (1906). - Frulistorfer: A. Seitz, Großschmett. Erde, v. 9, p. 440 (1911). - H. Stichẹl, Tierreich, Lief. 34, p. 39, 44 (1912).

subsp. o dana (God.) Morpho o., (Latreille \&) J. B. Godart, ut antea (1824). - Horsfield, Descr. Cat. Lep. Mus. E.-Ind. Comp., t. 6, f. 5, 5 a (1829). - Thaumantis o., Boisduval, Spec. Gén. Lép., v. 1, Expl. pl., p. 4 , t. 12 (8 B), f. 1 (1836). - E. Blanchard, Hist. An. Artic., v. 3, p. 456 (1840). - E. Doubleday, List Lep. Brit. Mus., pars 1, p. 115 (1844). - (E. Douhleday \&) Westwood, Gen. Diurn. Lep., v. 2, p. 336 (1851). - Chenu \& H. Lucas, Ene. Hist. Nat., Papill., p. 166 (1851). - Ménétriés, Enum. Corp. Anim. Mus. Petropol., Lep., pars 1, p. 37 (1855). - Horsfield \& F. Moore, Cat. Lep. Mus. E.-Ind. Comp., v. 1, p. 214 (1857). - Westwood, Trans. Ent.

Java,

Borneo,

Sumatra,

Nius,

Malayische

Halbinsel

West-Java 
Soc. London, a. ser., v. 4, p. 170 (1858). Herrich-Schäffer, Corr.-B1. Zool.-Min. Ver. Regensb., v. 19, p. 88 (1865). - W. F. Kirby, Cat. Diurn. Lep., p. 119 (1871). Thaumantias (!) o., W. F. Kirby, Entomologist, v. 10, p. 292 (18i7). - Thaumantis o., id., Sci. Proc. R. Dublin Soc., ser. 2, v. 2, p. 300 (1880). - Morpho (T.) o., Marshall \& Nicéville, Butt. Ind. Burm. Ceyl., v. 1. II, p. 306 (nota) (1883). - T. o., E. Haase, Corr.-Bl. Ent. Ver. Iris, v. 1, p. 104 (Organ. odor.) (1886). - O. Staudinger (\& Schatz), Exot. Schmett., v. 1, p. 203 (part., non t. 65: = T. o. cyclops) (1887). - E. Haase, Corr.-Bl. Ent. Ver. Iris, v. 1, p. 306 (Organ. odor.) (1S88). - F. Moone, Lep. Ind., v. 2, p. 185 (part.) (1895). - Fruhstorfer, Berlin. Ent. Zeit., v. 41, 1896, p. 301 (1897). - Eimer (\& Fickert), Entstehg. d. Art., pars 2, Orthogen., p. 155 (1897). - W. F. Kirby: Hübner \& Geyer, Samml. Exot. Schmett., Neue Ausg., v. 3, p. 47 (1901). T. o. o., H. Stichel, Gen. Ins., fasc. 36, p. 18 (1906). - Fruhstorfer: A. Seitz, Großschmett. Erde, v. 9, p. 440, 449 (1911). T. o. o. forma principalis, H. Stichel, Tierreich, Lief. 34, p. 45, 46 (1912).

o d a Hübn. T. o., Jac. Hübner, Samml. Exot. Schmett., v. 2, t. [274], f. 3,4 [1820-26]. - W. F. Kirby, Hübner \& Geyer, Samml. Exot. Schmett., Neue Ausg., v. 2, t. 274 (61), f. 3, 4 (1901).

Morpho $k l u g i u s$, Zinken-Sommer, Nov. Acta Ac. Leop., v. 15, p. 165 (part.), t. 15 , f. 12,13 (Q) (non f. 11, O').

forma albocostalis Frulist. T. odana forma a., Fruhstorfer: A. Seitz, Großschmett. Ende, v. 9, p. 449 (1911). - T. o. o. forma a., H. Sticliel, Tierreich, Lief. 34, p. 46 (1912).

subsp. we dana Fruhst. T. o. w., Fruhstor- Ost-Java

fer: A. Seitz, Großschmett. Erde, v. 9, p. 449 (1911). - H. Stichel, Tierreich, Lief. 34, p. 45, 46 (1912).

subsp. cy clops Röb. T. o. c., Röber, Soc. SüdEntom., v. 19, p. 105 (1904). - Id., l. c., Borneo v. 20 , p. $113(1905)$. - H. Stichel, Gen. Ins.. fasc. 36, p. 18 (1906). - Fruhstorfer: A. Seitz, Großschmett. Erde, v. 9, p. 441 (1911). - T. o. c. forma principalis, II. Stichel. Tierreich, Lief. 36, p. 45,46 (1912). - T. o. c., Moulton, Journ. Straits Branch Asiat. Soc., Nr. 65, p. 15 (1913).

T. o d a na, Herb. Druce, Proc. Zool. Soc. London, p. 341 (1873). - O. Staudinger (\& Schatz), Exot. Schmett., v. 1, p. 203 (part.), t. 65 (1887 \& 86). - A. Pagenstecher, Abh. Senckenb. Naturf. Ges., v. 23, p. 406 (1897). 
- Hanitsch, Journ. Straits Branch Asiat. Soc., $\mathrm{Nr} .34$, p. 83 (1900). - Shelford, l. c., Nr. 41, p. 108 (1904).

forma depupill a t a Fruhst. T. o. cyclops Typ.:

forma d., Fruhstorfer, Soc. Entom., v. 20, Südost-

p. 113 (1905). - H. Stichel, Gen. Ins., Borneo

fasc. 36 , p. 59 (1906). - T. o. c. "Form"

d., Fruhstorfer: A. Seitz, Großschmett. Frde, จ. 9, p. 441 (1911). - T. c. o. forma d., H. Stichel, Tiorreich, Lief. 34, p. 45,47 (1912). - Moulton, Journ. Straits Branch Asiat. Soc., Nr. 65, p. 15 (1913).

subsp. panwil a Fruhst. T. o. p., Fruhstor- Nord-

fer: A. Seitz, Großschmett. Erde, v. 9, p. Borneo

449 (1911). - T. o. cyclops forma p., H. (Brunei)

Stichel, Tierreich, Lief. 34, p. 47 (1912).

subsp. paramita Fruhst. T. o. nov. subsp., Sumatra, T. o. p., Fruhstorfer, Soc. Entom., v. 20, p. Simalur 113, 130 (1905). - T. o. p., H. Stichel, Gen. Gen. Ins., fase. 36, p. 59 (1906). - Fruhstorfer, Soc. Entom., v. 22, p. 107 (1907). Id.: A. Seitz, Großschmett., v. 9, p. 448 (1911). - H. Stichel, Tierreich, Lief. 34, p. 45, 47 (1912). - Eecke, Zool. Mededeel. Leiden, v. 4, p. 84 (1918). - W. Rothschild, Journ. Foder. Malay. Stat. Mus., v. 8, p. 153 (1920).

T. od $\operatorname{an} a$, L. Martin, Deutsch. Ent. Zeit. Iep., v. 8, p. 258 (1895). - Nicéville \& L. Martin, Journ. Asiat. Soc. Bengal, v. 64. II, 1895, p. 394 (1896).

${ }_{\text {„p }} i$ s h un a" (error!), Fruhstorfer: A. Seitz, Großschmett. Erde, v. 9, t. 105 b (1911).

subsp. yantiva Fruhst. T. o. y. Fruh- Nias storfer: A. Seitz, Großschmett. Ende, v. 9, p. 440 (1911). - H. Stichel, Tierreich, Lief. 34, p. 45,47 (1912).

subsp. pishuna Fruhst. T. o. p., Fruh- Malayische storfer, Soc. Entom., v. 20, p. 113 (1905). Halbinsel - T. o. o. forma p., H. Stichel, Gen. Ins., fase. 36, p. $59(1906),-T$. o. p., Fruhstorfer: A. Seitz, Großschmett. Erde, v. 9, p. 440 (non "pishuma", t. $105 \mathrm{~b}:=T$. o. paramita o) (1911). - T. o. pishuna, H. Stichel, Tierreich, Lief. 34, p. 45, 47 (1912). - Godfrey, Journ. Siam Soc. Nat. Hist. Suppl., v. 7, p. 262 (1930).

T. odana, Distant, Rhop. Malay., p. 427 (part.), t. 36, f. 3 (1886). - Flwes (\& 0. Möller), Trans. Ent. Soc. London, p. 282 (1888). - Pendlebury, Journ. Feder. Malay Stat. Mus., v. 11, p. 262 (1923).

\section{Sectio Epixanthi}

H. Stichel, Gen. Ins., fasc. 36, p. 18 (1906). - Id., Tierreich, v. 34, p. 48 (1912). 
noureddin Westw. T. n., (E. Doubleday \&) West- Malayische wood, Gen. Diurn. Lop., v. 2, p. 337 (part.) Halbinsel, (1851). - H. Stichel, Gon. Ins., fasc. 36, Sumatra, p. 18, 59 (1906). - Fruhstorfer: A. Seitz, Borneo Großschmett. Erde, v. 9, p. 439 (1911). II. Stichel, Tierreich, Lief. 34, p. 39, 48 (1912).

subsp. noureddin West. T. n., Westwood, Malayische ut antea (1851). - Id., Trans. Ent. Soc. Halbinsel London, n. ser., v. 4, p. 175, t. 20 , f. $1,1 \mathrm{a}$ (o'), f. 2, $2 a$ (Q) (1858). - Herrich-Schäffer, Corr.-Bl. Zool.-Min. Ver. Regensb., v. 19 , p. 88 (1865). - W. F. Kirby, Cat. Diurn. Lep., p. 120 (1871). - A. G. Butler, Trans. Linn. Soc. London, ser. 2, Zool., v. 1, p. 538 (1879). - W. F. Kirby, Sci. Proc. R. Dublin Soc., sel. 2, v. 2, p. 300 (1880). - Distant, Rhop. Malay., p. 78 , t. 6 , f. 3 ; t. 9 , f. 7 ; p. 426 (1882 \& 86). - G. F. L. Marshall \& Nicéville, Butt. Ind. Burm. Ceyl., v. 1. II, p. 306 (nota) (1883). - O. Staudinger (\& Schatz), Exot. Schmett. v. 1, p. 203 (part.) (1887). - Elwes (d O. Möller), Trans. Ent. Soc. London, p. 282 (1888). E. Haase, Corr.-Bl. Ent. Vor. Iris, p. 307 (Organ. odor:) (1888). - Elwes, Proc. Zool. Soc. London, p. 271 (1891). - Kringana n., F. Moove, Lep. Ind., v. 2, p. 185 (part.) (1895). - Thaumantis n. n., H. Stichel, Gen. Ins., fasc. 36, p. 18, 59 (1906). Fruhstorfer: A. Seitz, Großschmett. Erde, v. 9, p. 439 (non t. 101a: $=T$. n. sigirya, conf. p. 1135) (1911). - H. Stichel, Tierreich, Lief. 34 , p. 49 (cit. part. corrig.) (1912). - T. n., N. D. Riley \& Gabriel, Cat. Type Spec. Rhop. Brit. Mus., pars 1, p. 40 (1924).

subsp. sigir y a Fruhst. T. o. s., Fruhstorfer: Sumatra, A. Seitz, Großschmett. Erde, v. 9, p. 439 (1911). - H. Stichel, Tierreich, Lief. 36, p. 49 (1912).

? T. noureddin, Weyenbergh, Pet. Nouv. Entom., Année 6, p. 404 (1874). - „noureddin", Fruhstorfer: A. Seitz, Großschmett. Erde, v. 9, t. $10 \pm$ a $\left(\sigma^{\top}\right.$, O, corr. p. 1135: sigirya) (1911 \& 27).

subsp. $s u l t$ a $n u s$ Stich. T. n. s., H. Stichel, Süd-Borneo Gen. Ins., fase. 36, p. 19 , t. 3 , f. 4 (Q) (1906). - Fruhstorfer: A. Seitz, Großschmett. Erde, v. 9, p. 439 (1911). - H. Stichel, Tierreich, Lief. 34, p. 49, 50 (1912). T. noureddin, Horsfiold \& F. Moore, Cat. Iep. Mus. E.-Ind. Comp., v. 1, p. 215 (1857). - T. noureddia (!), Herb. Druce, Proc. Zool. Soc. London, p. 341 (1873). O. Staudinger (\& Schatz), Exot. Schmett., v. 1, p. 203 (part.) (1887). - Shelford, Journ. Straits Branch Asiat. Soc., Nr. 41, p. 
108 (part.) (1904). - L. Martin, Mitt. Münch. Ent. Ges., v. 1, p. 72 (Biol.) (1910).

- subsp. chatra Fruhst. N. n. c., Fruhstorfer, Nord-

Soc. Entom., v. 20, p. 113 (1905). - H. Borneo

Stichel, Gen. Ins., fasc. 36, p. 59 (1906). -

Fruhstorfer: A. Seitz, Großschmett. Erde, v. 9, p. 439 , t. $104 a$ (1912). - H. Stichel, Tierreich, Lief. 34, p. 49, 50 (1912). -

Moulton, Journ. Straits Branch Asiat. Soc.,

Nr. 65, p. 15 (part.) (1913).

T. noureddin, Distant \& Pryer, Ann. Mag. 4

Nat. Hist., ser. 5, v. 19, p. 51 (1887).

\section{Genus Zeuxidia Jac. Hübn.}

Jac. Hübner, Samml. Exot. Schmott., v. 2, Index, p. 2, t. [57] [1820-26]. - E. Doubleday, List Lep. Brit. Mus., pars 1, p. 114 (1844). - Westwood, Cab. Orient. Entom., p. 40 (conf. A. G. Butler, Trans. Ent. Soc. London, 1870, p. 481) (1848). - Z. (Aglaura Boisduval MS), (part.) (E. Doubleday \&) Westwood, Gen. Diurn. Lep., $\nabla_{0}$ 2, p. 327 (1851). - Z. (part.), Chenu \& H. Lucas, Enc. Hist. Nat., Papill., p. 162 (1851). - Z., Horsfield \& F. Mooro, Cat. Lep. Mus. E.-Ind. Comp., v. 1, p. 210 (1857). Herrich-Schäffer, Corr.-Bl. Zool.-Min. Ver. Regensb., v, 18, p. 112; v. 19, p. 88 (spec. omiss.) (1864 \& 65). - Hewitson, Exot. Butt., v. 3 [4], t. [53] Zeuxidia \& Aemona (1868). - A. G. Butler, Trans. Ent. Soe. Londou, p. 486 (Morphol.) (1870). - W. F. Kirby, Cat. Dium. Lep., p. 115, App., p. 646 (1871). - S. H. Scudder, Proc. Amer. Ac. Sei., v. 10, p. 292 (typ. Z. luxerii) (1875). - Wallace, Geogr. Distr. Anim., v. 1, p. $319(1876)$. - T. F. Kirby, Entomologist, v. 10 , p. 291 (1877). - F. Miüller, Jena. Zeit. Naturw., v. 11, p. 101 (Physiol.) (1877). - Id., Kosmos, Leipzig, v. 1, p. 394 (1877). - A. G. Butler, Trans. Linn. Soc. London, ser. 2, Zool., v. 1, p. 538 (1879). - W. F. Kirby, Sci. Proc. R. Dublin Soc.. ser. 2, v. 2, p. 300 (1850). - Zeuzidir, Zeuxidia, Zeuxidea. Distant, Rhop. Malay., p. 69,72 ; p. 424, 425 (eorr. p. 482) (1882 \& 86).

Zeuxidia, G. F. L. Marshall \& Nicéville, Butt. Ind. Burm. Ceyl., v. 1. II, p. 282; 285 (1883). - Gerhard, Berlin. Ent. Zeit., v. 27, p. 181 (distr. geogr.) (1883). - E. Haaso, Corr.-Bl. Ent. Ver. Iris, v. 1, p. 103, 308, 309 (organ. odor.) (1886 \& 8S). - Id., Zool. Anzeig., v. 11, p. 479 (1888). - Doherty, Journ. Asiat. Soc. Bongal, v. 55. II, p. 121 (1886). - G. Sempor: C. Semper, Reis. Arch. Philipp., pars 2. V, Sclmett., v. 1, p. 72 (1887). - O. Staudinger (\& Schatz), Exot. Schmett., v. 1, p. 188 (1887). - I. Glaser, Cat. Etymol. Col. Lep., p. 317 (spec. omiss.) (1887). Röber: O. Staudinger: \& Schatz, Exot. Schmett., v. 2, t. 31 (Morphol. Z. amethystus); p. 184, 186 (1885 \& 89). - Plateau (sec. E. Haase, 1888), Bull. Sxc. Ent. Belgique, v. 33, p. LXI (1889). B. Hagen, Tijdschr. Nederl. Aardr. Gen., ser. 2, v. 7, p. 200 (1890). - L. Martin, Berlin. Ent. Zcit., v. 35, p. 8 (1890). - F. Moore, Lep. Ind., V. 2, p. 172, 173 (Typ. Z. luxerii) (1895). - W. F. Kirby, Handb. Lep. (Allens Natural Libr.), v. 1, p. 198 (1894).Fruhstorfer, Ent. Zeit. Stettin, v. 55, p. 118 (1894). - Elera, Cat. Faun. Filipinas, v. 2, p. 270 (1895). - L. Martin, Deutsch. Ent. Zeit. Lep., v. 8, 1895, p. 261 (1896). - Nicéville \& L. Martin, Journ. Asiat. Soc, Bengal, v. 64. II, 1895, p. 393 (not. biol.) (1896). 
- Fimer (\& Fickert), Entstehg. d. Arten, pars 2, Orthogenesis, p. $178(1897)$ - W. F. Kirby: Hübner \& Geyer, Samml. Fxot. Schmett., Neue Ausg., v. 3, p. 46 (1901). - H. Stichel, Deutsch. Ent. Zeit. Iris, v. 15, p. 59 (1902). - Röber, Ent. Zeit. Stettin; v. 64, p. 338 (1903). - Xeuxidia (!), L. C. H. Young, Journ: Rombay Nat. Hist. Soc., v. 15, p. 298 (1903). - Zeuxidia (Sectio), Shelford, Journ. Straits Branch Asiat. Soc., Nr. 41, p. 106 (1904) - Z., Bingham, Faun. Brit. Ind., Butterfl., v. 1, p. 181, 186 (typ. Z. luxeri!) (1905). - H. Stichel, Gen. Ins., fasc. 36, p. 19, t. 1, f. 4 (Morphol.) (1906). - A. Pagenstecher, Geogr. Verbr. Schmett., p. 412 (1909). - Fruhstorfer: A. Seitz, Großschmett. Frde, v. 9, p. 433, p. 434 (Artengruppe) (1911). H. Stichel, Tierreich, Lief. 34, p. 4, 51, f. 1, 7 (Morphol.) (1912). - W. H. Evans, Journ. Bombay Nat. Hist. Soc., v. 21, p. 571 (1912). - Moulton, Journ. Straits Branch Asiat. Soc., Nr. 65, p. 15 (1913). - Eecko, Zool. Mededeel. Leiden, v. 4, p. 84 (1918). W. H. Fvans, Journ. Bombay Nat. Tist. Soa, v. 29, p. 793, 796 , t. 16, E9 (1923). - Zeunidia (!), id., 1. c., v. 29, p. 797 (nota) (1923). - Le Cerf, Enc. Ent., ser. B. 3, Lep., v. 1, p. 141 (Morphol.) (1926). - W. H. Evans, Identif. Ind. Butterfl, p. 84, 87, t. 16, E 9 (1927).

$N y m p h a l i s$ [Subgen.] (part.) Goeze, Entom. Beytr., v. 3. I, p. $226(1779)$. - N. (Gemmatus), (part.), D. H. Schneider, Nomencl. Entom., p. 37 (1785).

E q u e s Subgen. (part.), Gmelin-Linné, Syst. Nat., ed. 13, v. 1. V, p. $2243(1790)$. - E. (Achivus) (part.), D. H. Schneider, Nomencl. Entom., p. 33 (1785).

M o e ra (part.), Jac. Hübner, Verz. Schmett., p. 51 [1818]. - S. H. Scudder, Proc. Amer. Ac. Sci., v. 10, p. 220 (nom. praeoce.) (1875).

Morpho (part.), Latreille, Enc. Méth., v. 9, p. 11, 435 (1819 \& 24). - J. B. Godart, 1. c., p. 439 (1824). - Verloren, Cat. Ins. Lep. Cramer, v. 2, p. 200 (1837). - Thon, Thon \& Reichenbach, Naturg. Schmetterl., p. 76 (1837 \& 38).

A mathusia (part.), A. G. Butler, Cat. Diurn. Lep. Fabr., p. 45 (1869).

A gla ura (Boisduval MS, sec. Westwood, 1851), S. H. Scudder, Proc. Amer. Ac. Sci., v. 10, p. 105 (1875).

A $m a x$ idia (Subgen.), O. Staudinger (\& Schatz), Exot. Schmett., v. 1, p. 188 (1887). - R. Haase, Corr,-Bl. Ent. Ver. Iris, v. 1, p. 308 (1888). - A., F. Moore, Lep. Ind., v. 2, p. 178 (1895). - A. (Subgen.), Nicéville \& L. Martin, Journ. Asiat. Soc. Bengal, v. 64. II, 1895, p. 393 (1896). A., Harlert, Nov. Zool., v, 9, p. 232 (1902). - H. Stichel, Deutsch. Ent. Zeit. Iris, v. 15, p. 59 (nota) (1902). Röber, Ent. Zeit. Stettin, v. 64, p. 339 (nom, vanum) (1903). - A. (Sectio), Shelford, Journ. Straits Branch Asiat. Soc., Nr. 41, p. 106, 107 (1904). - A., Fruhstorfer, Berlin. Ent. Zeit., v. 49, p. 190 (1904); id.: A. Seitz, Großschmett. Erde, p. 437 (Artengruppe) (1911). - Oh. Oberthür, Journ. Feder. Malay Stat. Mus., v. 8, p. 153 (1920). N. D. Rilev \& Gabriel, Cat. Type Spec. Rhop. Brit. Mus., pars 1, p. 9 (1924).

$Z$ e $u x a l t i s$ (Subgen.), A. G. Butler, Ann. Mag. Nat. Hist., ser. 6, v. 19 , p. 469 (1897). $-Z$. (Sectio), Shelford, Journ. Straits Branch Asiat. Soc., Nr. 41, p. 106 (1904). 


\section{Sectio Glaucopferofi}

H. Stichel, Gen. Ins., fasc. 36, p. 21 (1906). - Id., Tierreich, Lief. 34 , p. 55 (1912).

amethystus Butl. Z. a., A. G. Butler, Proc. Zool. Soc. Burma,

London, p. 485 (1865). - H. Stichel, Gen.

Ins., fasc. 36, p. 21 (1906). - Fruhstorfer:

A. Seitz, Großschmett. Erde, v. 9, p. 435

(1911). - H. Stichel, Tierreich, Lief. 34,

p. 54, 55 (1912). - Godfrey, Journ. Siam

Soc. Nat. Hist., Suppl., v. 7, p. 260 (1930).

Tenasserim,

Malayische

Halbinsel,

Sumatra,

Borneo,

Philippinen

subsp. $a$ meth y st us Butl. Z. a., A. G. But- Malayische ler, ut antea (1865). - Id., Lep. Exot., p. Halbinsel,

29 (1870). - W. F. Kirby, Cat. Diurn. Lep., Sumatra, p. 116 (1871). - A. G. Butler, Trans. Bangka

Linn. Soc. London, ser. 2, Zool., v. 1, p. 538

(1879). - Z. a., Z. a. var., Distant, Rhop. Malay., p. 72 (cit. part.), t. 7 , f. 1 ( ( $)$, f. 2 (P); p. 424 , t. 38 , f. 5 ( (") $(1882$ \& 86). - G. F. L. Marshall \& Nicéville, Butt. Ind.

Burm. Ceyl., v. "1. II, p. 86 (part.) (1883). O. Staudinger (\& Schatz), Exot. Schmett., v. 1, t. 63, p. 188 (part.) (1886 \& 87). Eimer (\& Fickert), Entstehg. d. Arten, pars 2, Orthogen., p. 155, 235, 237 (1887). - E. Haase, Corr.-Bl. Ent. Ver. Iris, v. 1, p. 309 (Organ. odor.) (1888). - Elwes (\& O. Möller), Trans. Ent. Soc. London, p. 282 (1888). - O. Staudinger, Deutsch. Ent. Zeit. Lep., v. 2, p. 41 (1889). - B. Hagen, Tijdschr. Noderl. Aardr. Gen., ser. 2, v. 7, p. 200 (1890). - Elwes, Proc. Zool. Soc. London, p. 270 (1891). - B. Hagen, Berlin. Ent. Zeit., v. 37, p. 144 (1892). - F. Moore, Lep. Ind., v. 2, p. 175 (1895). - Nicéville \& L. Martin, Journ. Asiat. Soc. Bengal, v. 64. ПI, 1895, p. 392 (1896). - L. Martin, Deutsch. Ent. Zeit. Lep., v. 8, 1895, p. 261 (1896). - Z. a. a., H. Stichel, Gen. Ins., fasc. 36, p. 21 (1906). - Fruhstorfer: A. Seitz, Großsichmott. Erde, v. 9, p. 435 (1911). - H. Stichel, Tierreich, Lief. 34 , p. 56, 57 (1912). - Pendlebury, Journ. Feder. Malay Stat. Mus., v. 11, p. 35 (1923). - Z. a., N. D. Riley \& Gabriel, Cut. Type Spec. Rhop. Brit. Mus, pars 1, p. 7 (1924). Z. a. a., Godfrey, Journ. Siam Soc. Nat. Hist., Suppl., v. 7, p. 260 (1930).

forma prodigios a Frulst. Z. a. a. forma Typ.: p., Fruhstorfer: A. Seitz, Großschmett. Erde, P Sumatra v. 9, p. 435 (1911). - H. Stichel, Tierreich, Lief. 34 , p. 57 (1912).

subsp. $M a s 0 n_{i}$ F. Moore Z. m., F. Moore, Süd-Burma, Proc. Zool. Soc. London, p. 826 () (1878). Tenasserim, - G. F. L. Marshall, Journ. Asiat. Soc. Siam Bengal, v. 51. II, p. 39 (1882). - G. F. L. Marshall \& Nicéville, Butt. Ind. Burm. Ceyl., v. 1. II, p. 286 (1883). - Elwes \& 
Nicéville, Journ. Asiat. Soc. Bengal, v. 55. II, 1886, p. 419 (1887). - F. Moore, Lep. Ind., v. 2 , p. 174, t. 144 , f. $1,1 a_{*}$ b $(0$, , Q $)(1895)$. - Bingham, Faun. Brit. Ind., Lep., v. 1, p. 186 , t. 4 , f. 29 ( $\left.\sigma^{7}\right)(1905)$. - Z. amethys tus m., H. Stichel, Gen. Ins., fasc. 36, p. 21 (1906). - Frubstorfer: A. Seitz, Großschmett. Erde, v. 9, p. 435 (1911). - H. Stichel, Tierreich, Lief. 34, p. 57, 58 (1912). - Z. a. "race" m., W. H. Evans, Journ. Bombay Nat. Hist. Soe., v. 21, p. 571 (1912). - Z. m., Ollenbach, 1. c., v. 26 , p. 869 (not. biol.) (1918). - Z, amethystus m., id., 1. c., v. 27 , p. 887 (1921). - W. H. Evans, Journ. Bombay Nat. Hist. Soc., v. 29 , p. 796, t. 16 , f. E 9.1 (1923). - Z. m., N. D. Riley \& Gabriel, Cat. Type Spec. Rhop. Brit. Mus., pars 1, p. 35 (1924). - Z. amethystus m., W. H. Evans, Identif. Ind. Butterfl., p. 87, t. 16, fig. E 9.1 (1927). - Godfrey, Journ. Siam Soc. Nat. Hist., v. 7 , p. 260 (1930).

subsp. Wa lla cei C. \& R. Feld. Z. w., O. Borneo

\& R. Felder, Reiso Novara, v. 2. II, p. 461, t. 62, (f. $3\left(\sigma^{7}\right)(1866)$. - W. F. Kirby, Cat. Diurn. Lep., p. 115 (1871). - Herb. Druce, Proc. Zool. Soc. London, p. 341 (1873). - Z. amethystus var. $w ., \quad 0$. Staudinger, Deutsch. Ent. Zeit. Lep., v. 2, p. 41 (1889). - Z . w., F. Moore, Lep. Ind., v. 2, p. 175 (1895). - Shelford, Journ. Straits Branch Asiat. Soc., Nr. 41, p. 106 (1904). - Z. amethystus w., H. Stichel, Gen. Ins., fasc. 36, p. 21 (1906). - Fruhstorfer: A. Seitz, Großschmett. Erde, จ. 9, p. 435, t. $102 \mathrm{c}\left(\mathrm{O}^{7}\right)$ (1911). - H. Stichel, Tierreich, Lief. 34, p. 57 (1912). - Moulton, Journ. Straits Branch Asiat. Soc., Nr. 65, p. 15 (1913).

Z. a methyst us, A. G. Butler, Lop. Exot., p. 29 (1870). - W. F. Kirby, Oat. Diurn. Lep., App., p. 646 (1871). - Distant, Rhop. Malay., p. 72 (in cit.) (1882). - G. F. L. Marshall \& Nicéville, Butt. Ind. Burm. Ceyl., p. 286 (part.) (1883). - O. Staudinger (\& Schatz), Exot. Schmett., v. 1, p. 189 (part.) (1887). - Shelford, Journ. Straits Branch Asiat. Soo., Nr. 41, p. 106 (1904). Moulton, Entomologist, v. 45, p. 247 (1913).

subsp, a methystina H. Stich. $Z$. amethystus var. amathystina, O. Staudinger (\& Bang-Haas), Lepidopt-Listo 34, p. 24 (nom. nud.) (1890). - Z . amethystus amethystina, H. Stichel, Gen. Ins., fasc. 36 , p. 21 (1906). - Fruhstorfer: A. Seitz, Großschmett. Erde, v. 9 , p. 435 (1911). - H. Stichel, Tierreich, Lief. 34 , p. 57 (1912).

Z. a methystus, G. Semper: G. Semper, Reis. Arch. Philippin., pars 2. V, Schmett., v. 1 , p. 73, p. 72, fig. (Morphol.) (1887). O. Staudinger (\& Schatz), Exot. Schmett.,

Mindanao, Camiguin 
v. 1, p. 188 (part.) (1887). - Elera, Cat. Faun. Filipin., v. 2, p. 270 (sine cit.) (1895). subsp. victrix O. Stauding. Z. $v$. (Z. amethystus var.?), O. Staudinger, Deutsch. Ent. Zeit. Lep., v. 2, p. 41 (1889). - Z. v., G. Semper: C. Semper, Reis. Arch. Philippin., v. 5, p. 332 (1892). - F. Moore, Irep. Ind., v. 2, p. 176 (1895). - Elera, Cat. Faun. Filipin., v. 2, p. 270 (1895). - Z. amethystus v., H. Stichel, Gen. Ins., fasc. $36, \mathrm{p}$. 21 (1906). - Fruhstorfer: A. Soitz, Großschmett. Erde, v. 9, p. 435 , t. $103 \mathrm{c}\left(0^{7}\right.$, O) (1911). - H. Stichel, Tierreich, Lief. 34, p. 56,58 (1912).

Doubledaii Westw. Z. d., (E. Doubleday \&) West- Borneo, wood, Gen. Diurn. Lep., v. 2, p. 329 (1851). Billiton, - H. Stichel, Gen. Ins., fasc. 36, p. 22 (1906). - Fruhstorfer: A. Seitz, Großschmett. Erde, v. 9, p. 435 (1911), - H. Stichel, Tierreich, Lief. 34, p. 54, 59 (1912).

- subsp. Doubledaii Westw. Z. d., Westwood, ut antea (nota) (Patria falsa?: India) (1851). - Z. doubledayi, Chenu \& H. Lucas, Enc. Hist. Nat., Papill., p. 163 (1851). Herrich-Schäffer, Corr.-BI. Zool--Min. Ver. Regensb., v. 19 , p. 88 (1865). - Z. doubledaii, W. I. Kirby, Cat. Diurn. Lep., p. 115 (1871). - G. F. L. Marshall \& Nicéville, Butt. Ind. Burm. Ceyl., v. 1. II, p. 287, fig. (Q, copia figurae Doubleday - Westwood) (1883). - O. Staudinger (\& Schatz), Exot. Schmett., v. 1, p. 189 (1887). - Z. doubledayi, F. Mooro, Isop. Ind., v. 2, p. 175 (part.) (1895). - Z. doubledai, Shelford, Journ. Straits Branch Asiat. Soc., Nr. 41, p. 106 (1904). - H. Stichel, Gen. Ins., fasc. 36, p. 22 (part.) (1906). - Fruhstorfer, Deutscli. Ent. Zeit. Iris, v. 19, p. 105 (1906). - Id., Sac. Entom., v. 22, p. 108 (1907). Id.: A. Seitz, Großschmett. Erde, v. 9, p. 435 , t. 103 c (o', 옹 doubledayi). — Z. doubledayi, Moulton, Intomologist, v. 45, p. 247 (1912). - Id., Journ. Straits Branch Asiat. Soc., Ni. 65, p. 16 (1913). - T. H. Evans, Journ. Bombay Nat. Hist. Soc., v. 22, p. 762 (1914). - $Z$. doubledai, N. D. Riley \& Gabriel, Cat. Type Spec. Rhop. Brit. Mus., pars 1, p. 18 (1924).

Z. $l$ u $x$ er $i \mathrm{i}$ (non Hübner), E. Doubleday (\& Westwood), Gen. Diurn. Lep., v. 1, t. 52, f. 1 (ㅇ) (1849). - Z. luxeri, Chenu \& H. Lucas, Enc. Hist. Nat., Papill., t. 36, f. 1 (1851).

forma Pryeri A. G. Butl. Z. (Zeuxaltis) p., Typ.: NordA. G. Butler, Ann. Mag. Nat. Hist., ser. 6, v. Borneo 19 , p. 470 (1897). — Zeuxidia p., Shelford,

\section{Palawan}

(Paragua)

Sumatra,

Bangka,

Malayische

Tralbinsel,

Tenasserim,

Siam

Nord- und

Ost-Borneo 
Journ. Straits Branch Asiat. Soc., Nr. 41, p. 106 (1904). - T. d. p., Fruhstorfer: A. Seitz, Großschmett. Erde, v. 9, p. 436 (monströse Folm) (1911).

subsp. II or sfieldii C. \& R. Feld. Z. h., SüdostC. \& R. Felder, Reise Novara, v. 2. II, p. 460, Borneo t. 62, f. 4 (1866). - W. F. Kirby, Cat. Diurn. Lep., p. 115 (1871). - Herb. Druce, Proc. Zool. Soc. London, p. 340 (1873). O. Staudinger (\& Schatz), Exot. Schmett., v. 1, p. 189 (1887). - F. Moore, Lep. Ind., v. 2, p. 176 (1895). - Z. horsfieldi, Fruhstorfer, Ent. Nachr., v. 19, p. 259 (1893). - Id., Berlin. Ent. Zeit., v. 40, Sitzsber., p. (18) (1895). - Z. doubledaii h., id., Deutsch. Ent. Zeit. Iris, v. 19, p. 105 (1906). - Id., Soc. Entom., v. 22, p. 106 (1907). - Id.: A. Seitz, Großschmett. Erde, v. 9, p. 436 (1911). - Z. d. horsfieldii, H. Stichel, Tierreich, Lief. 34, p. 61, 62, f. 8 (ठ) $(1912)$.

$Z$. Do u b ledayi (non Z. doubledaii Westw.), Honrath, Berlin. Ent. Zeit., v. 32, p. 253 (Q) (1888). - Z. doubledaii, H. Stichel, Gen. Ins., fase. 36 , p. 22 (in cit.) (1906).

Honrathi Stauding. $Z$. h., O. Staudinger (\& Bang-Haas), Lepid.-Liste Nr. 38, p. 30 (ex errore pro $Z$. dohrni, conf. Fruhstorfer, Berlin. Ent. Zeit., v. 40, Sitzgsber., p. (18), 1895) (1895).

subsp. s u matrana Fruhst. Z. d. s., Fruh- Sumatra, storfer, Deutsch. Ent. Zeit. Iris, v. 19, p. Simalur. 105 (1906). - Z . d. s., Z. d. nicévillei forma ? Billiton s., id., Soc. Entom., v. 22, p. 106, 107 (1907). -Z.d.s., id.: A. Seitz, Großschmett. Erde, v. 9, p. 436 (1911). - Z. d. s. forma principalis, H. Stichel, Tierreich, Licf. 34, p. 61, 62 (cit. part.) (1912). - Eecke, Zool. M fededeel. Leiden, v. 4, p. 84.

? Z. Hor sfieldi (non Felder), F. D. Godman \& O. Salvin, Proc. Zool. Soc. Luondon, p. 639 (1878). - Z. horsfieldii, P. C. T. Snellen, Tijdschr. Ent., v. 33, p. $282(1890)$. - Id., Not. Leyden Mus., v. 13, p. 133 (sec. Godman \& Salvin, 1878) (1891).

Z. Nicévillei (part.), Fruhstorfer, Ent. Nachr., v. 21, p. 196 (1895). - H. Stichel, Gen. Ins., fasc. 36, p. 22 (1906).

?Z. Doubledai $i$ L. Martin, Deutsch. Ent. Zeit. Lep., v. 8, p. 262 (1896). $-Z$. d. (part.), H. Stichel, Gen. Ins., fasc. $36, \mathrm{p}$. $22,(1906)$.

subsp. cher s one sia Fruhst. Z. $d$. c., Fruh- Malayische storfer, Soc. Entom., v. 22, p. 106, 107 Halbinsel, (1905). - Id., Deutsch. Ent. Zcit. Iris, Tenasserim, v. 19 , p. 105 (1906). - Id., Soc. Entom., Siam, v. 22, p. 106 (1907). - Id., A. Seitz, SüdGroBschmett. Erde, v. 9, p. 436 (1911). - Burma Z. doubledaii sumatrana forma c., H. Stichel, 
Tierreich, Lief. 34, p. 63 (1911). - Z. doubledayi "race" c., W. H. Evans, Journ. Bombay Nat. Hist. Soc., v. 22, 1913, p. 762 (1914). - Id., W. H. Evans, 1. c., v. 29, p. 796 (1923). - Id., Identif. Ind. Butterfl., p. 87 (1927). - Z. doubledayi c., Godfrey, Journ. Siam Soc. Nat. Hist., Suppl., v. 7, p. 261 (sine cit.) (1930).

Z. Doubledaii (non Westwood), Distant, Rhop. Malay., p. 424, fig. 124 ( $\sigma^{7}$ ) (non $Q$, t. 38 , f. $6:=Z$. nicevillei) $(1886)$. $-Z$. doubledayi, Elwes (\& O. Möller), Trans. Ent. Soc. Ifondon, p. 282 (1888). - F. Moore, Lep. Ind., v. 2, p. 175 (part.) (1895). - W. H. Evans, Journ. Bombay Nat. Hist. Soc., v. 21, p. 571 (1912).

subsp. a $n a x$ i l a Fruhst. Z. d. a., Fruhstor- Bangka

fer: A. Seitz, Großschmett. Erde, v. 9, p. 437 (1911). - H. Stichel, Tierreich, Lief. 34, p. $61,63(1912)$.

Z. Doubledaii, B. Hagen, Berlin. Ent. Zeit., v. 37 , p. 141 (1892).

Nicevillei Fruhst. (spec. dubia) Z. n., Fruhstorfer, Sumatra, Ent. Nachr., v. 21, p. 196 ( $Z$. doubledaii Malayische forma loci) (1895). - Z. n., L. Martin, Deutsch. Ent. Zoit. Lep., v. 8, p. 262 (?=Z. doubledaii) (1896). - L. Martin \& Nicéville, Journ. Asiat. Soc. Bengal, v. 64. II, 1895 , p. 392 (1896). - Z. n., Z. n. nov. subsp., Fruhstorfer, Deutsch. Ent. Zeit. Iris, v. 19 , p. 105 (1905). - Z. n., H. Stichel, Gen. Ins., fasc. 36 , p. 22 , t. 3, f. $5\left(\sigma^{7}\right)$ (cit. part.) (1906). - Z.n.n., Z. doubledaii n., Fruhstorfer, Soc. Entom., v. 22, p. 106, 107 (1907). - Z. d. n., id.: A. Seitz, Großschmett. Erde, v. 9, p. 436, t. 102 c (1911). Z. n., H. Stichel, Tierroich, Lief. 34, p. 54, 63 (1912). - Z. doubledaii n., IV. Rothschild, Journ. Feder. Malay Stat. Mus., v. 8, p. $153(1920)$.

? Z. Dou $u$ ledayi, Distant, Rhop. Malay., p. 424 , t. 38 , f. 6 (ㅇ) (non $\sigma^{*}$, fig. 124: = Z. doubledaii chersonesia) (1886). - ? Z doubledai, P. C. T. Snellen, Tijdschr. Ent., v. 33, p. $282(1890)$. — ? Id., Not. Leyden Mus., v. 13, p. 133 (1891). - F. Moore, Lep. Ind., v. 2, p. 175 (in cit.) (1893).

luxeril Jac. Hübn. $Z$. l., Jac. Hübner, Samml. Exot, Java, Schmett., v. 2, t. $[57][1820-26]$ - H. Bali, Stichel, Gen. Ins., fasc. 36, p. 22 (1906). - Sumatra Fruhstorfer: A. Seitz, GroBschmett. Erde, v. 9, p. 437 (1911). - H. Stichel, Tierreich, Lief. 34, p. 55,63 (1912).

subsp. $l u x$ eri $i$ Jac. Hübner $Z$. $l$., Jac. Java, Bali Hübner, ut antea $[1820-26]$. - E. Doubleday, List Lep. Brit. Mus., pars 1, p. 114 (1844). - Westwood, Cab. Orient. Entom. t. 19, f. 5 (1848). - Chenu \& H. Lucas, Ene. Hist. Nat. Papill., p. 163 (non t. 36, $f$. 
1: = Z. doubledaii Q) (1851). - (E. Doubleday \&) Westwood, Gen. Diurn. Lep., v. 2, p. 329 (1851). - Z. luxeri, Horsfield \& F. Moore, Cat. Lep. Mus. E.-Ind. Comp., p. 210 (1857). - Z. luxerii, Herrich-Schäffer, Corr.-Bl. Zool.-Min. Ver. Regensb., v. 19, p. 88 (1865). - Z. luxerrei (!), O. \& R. Felder, Reise Novara, v. 2. II, p. 460 (1866). - Z. Iuxerii, W. F. Kirby, Cat. Diurn. Lep., p. 115 (1871). - Id., Entomologist, マ. 10, p. 291 (1877). - Id., Sci. Proc. R. Dublin Soc., ser. 2, v. 2, p. $300(1880)$. - G. F. L. Marshall \& Nicéville, Butt. Ind. Burm. Ceyl., v. 1. II, p. 297 (nota) (1883). - F. Haase, Corr.-Bl. Ent. Ver. Iris, v. 1, p. 103 (Organ. odor.) (1886). - O. Staudinger (\& Schatz), Exot. Selimett., v. 1, p. 189 (1887). - Fruhstorfer, Entom. Nachr., v. 19, p. 259 (1893). - F. Moore, Lep. Ind, v. 2, p. 175 (1895).

- Z. luxeri, Fruhstorfer, Berlin. Ent. Zeit., v. 41, 1896, p. 301 (1897). - Z. iuxerii, Nicéville \& Elwes, Journ. Asiat. Soc. Bengal, v. 66. II, 1897 , p. 682 (1898). - W. F. Kirby: Hübner \& Geyer, Samml. Exot. Schmett., v. 2, t. 270 (57), f. 1, 2; v. 3, p. 46 (1898 \& 1901). - Z. l. l., H. Stichel, Gen. Ins., fusc. 36, p. 23 , t. 3 , f. 6 () (1906). - Z. luxeri l., Z. l., Fruhstorfer: A. Seitz, Großschmett. Ende, v. 9, p. 437,449 (larva) (1911). - Z. luxerii l., H. Stichel, Tierreich, Lief. 34, p. 65 (cit. ex parte corrig.) (1912).

Boisduvalii, Westw. Z. b., (Aglaura nep̧us Boisduval MS) (E. Doubleday \&) Westwood, Gen. Diurn. Lep., v. 2, p. 329 (Q) (1851). - Chenu \& H. Lucas, Enc. Hist. Nat., Pap., p. 163 (1851). - Herrich-Schäffer, Corr.-Bl. Zool.-Min. Ter. Regensb., v. 19 , p. 88 (1865). - W. F. Kirby, Cat. Diurn. Lep., p. 116 (1871). - Fruhstorfer, Ent. Nachr., v. 19, p. 259 (1893).

forma prodigiosa Fruhst. Z. luxeri p., Typ.: Java Fruhstorfer: A. Seitz, Großschmett. Erde, v. 9, p. 437 (1911).

subsp. suc culenta H. Stich. Z. l. s., H. NordostStichel, Gen. Ins., fasc. 36, p. 22 (1906). Sumatra - Z. luxeri s., Fruhstorfer: A. Seitz, Großschmett. Erde, v. 9, p. 437 (1911). - H. Stichel, Tierreich, Lief. 34, p. 64, 65 (1912).

Z. $l$ u x e ri $i$, Nicéville \& Elwes, Journ. Asiat. Soc. Bengal, т. 64. II, 1894, p. 393 (1895).

Fruhst. Z. d., Frubstorfer, Ent. Nachr., v. Java 19, p. 257 (1893). - Id., Ent. Zeit. Stettin, v. 55, p. 116, t. 3, f. 2 ; t. 4, f. $2-6$ (1894). - Id., Berlin. Ent. Zeit., "จ. 40, Sitzgsber., p. (18) (1895). - P. C. T. Snellen, Tijdschr. Entom., v. 38, p. 16 (1895). - Fruhstorfer, Berlin. Ent. Zeit., v. 41, p. 301 (1897). - H. Stichel, Gen. Ins., fasc. 36, 
p. 23 (1906). - Fruhstorfer: A. Seitz, Großsclimett. Erde, v. 9, p. 434, t. $102 \mathrm{c}$ (O) (1911). - H. Stichel, Tierreich, Lief. 34 , p. 54, 65 (1912).

Z. horsfieldi $i$ (vera) (non Felder!), 0 . Staudinger (\& Bang-Haas), Lepid.-Liste Nr. 38 , p. 30 (1895).

Semperi O. \& R. Feld. Z. s., O. \& R. Felder, Wien. Philippinen

Ent. Monatschr., v. 5, p. 304 (1861). - Fruhstorfer: A. Seitz, Grolischmett. Erde, v. 9, p. 435 (1911). - H. Stichel, Tierreich, Lief. 34 , p. 55,66 (1912).

subsp. Semperi C. \& R. Feld. Z. s., C. Luzon \& R. Felder, ut antea (1861). - HerrichSchäffer, Corr.-Bl. Zool.-Mín. Ver. Regensb., v. 19 , p. 88 (1865). - O. \& R. Felder, Reise Nova ra, v. 2. II, p. 460 , t. 62 , f. 1, 2 (1866). - W. F. Kirby, Cat. Diurn. Lep., p. 116 (1871). - G. Semper: O. Semper, Reis. Arch. Philippin., pars 2. V, Sclimett., p. 72, 332 (distrib. part.) (1887 \& 92). - O. Staudinger (\& Schatz), Exot. Schmett,, v. 1, p. 189 (1887). - F. Moore, Lep. Ind., v. 2, p. 176 (1895). - Elera, Cat. Faun. Filipin., v. 2, p. 270 (? part.) (1895). - H. Stichel, Gen. Ins., fasc. 36 , p. 23 (1906). - Z. s. s., Fruhstorfer: A. Seitz, Großschmett. Erde, v. 9 , p. 435 (1911). - Z. s. forma principalis, H. Stichol, Tierroich, Lief. 34, p. 66 (1912). subsp. the ri o narca Fruhst. Z. s. t., Fruhstorfer: A. Seitz, Großschmett. Erde, v. 9, p. 435 (1911). - Z. s. - forma t., H. Stichel, Tierreich, Lief. 34 , p. 68 (1912).

subsp. excels a W. Rothsch. Z. 8. e., W. Negros

Rothschild, Nov. Zool., v. 23, p. 308 (1916).

sibulana Honr. Z. s., Honrath, Berlin. Ent. Zeit., Mindanao

v. 28 , p. 205 , t. 2 , f. 2 , t. 3 , f. 2 a $\left(\sigma^{7}\right)$; t. 4, f. 2 b (o) (1884). - G. Semper: C. Semper, Reis, Archip. Philippin., pars 2. V, 'Schmett., v. 1, p. 73 (1887). - O. Staudinger (\& Schatz), Exot. Schmett., v. 1, p. 189 (1887). - Fruhstorfer, Ent. Nachr., v. 19, p. 259 (1893). - F. Moore, Lep. Ind., v. 2, p. 176 (1895). - Elera, Cat. Faun. Filipin., v. 2 , p. 270 (1895). - H. Stichel, Gen, Ins., fasc. 36 , p. 23 (1906). - Fruhstorfer: A. Seitz, Großschmott. Erde, v. 9, p. 435 (1911). - H. Stichel, Tierreich, Lief. 34, p. 55, 68 (in cit. falso Z. zibulana Fruhst.) (1912). - N. D. Riley \& Gabriel, Cat. Type Spec. Rhop. Brit. Mus., pars 1, p. 53 (1924).

Mindoro

\section{Sectio Poliodermi}

H. Stichel, Gen. Ins., fasc. 36, p. 23 (1906). - Id., Tierreich, Lief. 34 , p. 69 (1912).

aurelia (Cram.) Pap. aurelius, P. Cramer, Pap. Sumatra, Exot., v. 2, p. 147 (1777). - Zeuxidia a., Simalur, Fruhstorfer, Berlin. Ent. Zeit., v. 49, p. 190 Malayische 
(1904). - Z. aurelia, H. Stichel, Gen. Ins., fasc. 36, p. 23 (1906). - Z. aurelius, Fruhstorfer: A. Seitz, Großschmett. Erde, v. 9, p. 437 (1911). - Z. aurelia, H. Stichel, Tierreich, Lief. 34, p. 54, 69 (1912).

subsp. a urelia (Oram.) „Aurelius", Papilio Sumatra, aurelius, P. Oramor, Pap. Exot., v. 2, p. 110, Simalur, t. 168 , f. A, B (Q); p. 147 (1877). - Pap. Malayische (Nymphalis) a., Groeze, Ent. Beytr., v. 3. I, p. 293 (1779). - P. a., J. C. Fabricius, Spec. Ins., v. 2, p. 21 (1781). - C. Stoll, Essai Syst. Lep.: P. Cramer, v. 4, App., p. 8 (1782). - J. O. Fabricius, Mant. Ins,, v. 2, p. 11 (1787). - P. (Eques Achivus) a., P. (Nymphalis Gemmatus) a., [D. H. Schneider] Nomencl. Entom., p. 33, 37 (1785). $P$. a., (Jablonsky \&) Herbst, Naturs. Ins., Schmett., v. 3, p. 119 , t. 38, f. 1, 2 (क) (1788). - P. (Eques) a., Gmolin-Linné, Syst. Nat., ed. 13, v. 1. V, p. 2243 (1790). P.a., Jung, Alplaab. Verz. Schmett., v. 1, p. 68 (1791). - J. C. Fabricius, Ent. Syst., v. 3. I, p. 71 (1793). - Latham \& H. Davies: J. R. Forster, Zool. Index, ed. 2, pars 3, p. 23 (1795). - G. Shaw, Natural. Miscell., v. 14, t. 587 (Q); Index (1803). - Turton, Gen. Syst. Nat. Linné, v. 3. II, p. 82 (1806). Moera aurelia, Jac. Hübner, Verz. Schmett., p. 51 [1818]. - Morpho aurelius, (Latreille \&) J. B. Godart, Enc. Méth., v. 9, p. 439 (1824). - Verloren, Cat. Ins. Lep. Cramer, v. 1 , p. 70 ; v. 2, p. 201 (1837). - Thon, Thon \& Reichenbach, Naturg. Schmett., p. 76, t. 38 , f. 550,551 (1837 \& 38). - Zeuxidia a., (E. Doubleday \&) Westwood, Gen. Diurn. Lap., v. 2, p. 329 (1851). - Chenu \& H. Lucas, Enc. Hist. Nat., Papill., p. 163 (1851). - Herrich-Schäffer, Corr.-Bl. Zool.Min. Ver. Regensb., v. 19, p. 88 (1865). Amathusia a., A. G. Butler, Cat. Diurn. Lep. Fabr., p. 45 (1869). - Zeuxidia a., W. F. Kirby, Cat. Diurn. Lep., p. 116 (1871). Id., Entomologist, v. 10, p. 291 (1877). E. Haase, Corr.-Bl. Ent. Ver. Iris, v. 1, p. 103 (1886). - Zeuxidea (!) a., Zeuxidia a., Distant, Rhop. Malay., p. 425,482 , t. 37, f. 1 (1886). - Amathusia (Amaxidia) a., (part.), Zeuxidia a., O. Staudinger (\& Schatz), Exot. Schmett., v. 1, p. 188, 189 (1887). - Amaxidia (Zeuxidia) a., Honrath, Berlin. Ent. Zeit., v. 31, p. 352 (1887). - Z. a., Elwes \& Nicéville, Trans. Ent. Soc. London, p. 282 (1888). - Elwes, Proc. Zool. Soc. London, p. 570 (1891). - Amaxidia a., F. Moore, Lep. Ind., v. 2, p. 179 (eit. part.) (1895). - Zeuxidia (A.) a., L. Martin, Deutsch. Ent. Zeit. Lep., v. 8, 1895 , p. 262 (1896). Nicóville \& L. Martin, Journ. Asiat. Soc. Bengal, v. 64. II, 1895 , p. 393 (1896). -

Halbinsel, Mergui, Borneo

Halbinsel, Mergui 
Z. a., Eimer (\& Fickert), Entstehg. 'd. Arten, pars 2, Orthogenesis, p. 117 (1897). E. Hartert, Nov. Zool., v. 9, p. 232 (1902). Amaxidia a. a., Fruhstorfer, Berlin. Ent. Zeit., v. 49, p. 190 (1904). - Zeuxidia $a$. a., H. Stichel, Gen. Ins., fasc. 36, p. 23 (1906). - Z. a., Fruhstorfer, Soc. Entom., v. 22 , p. 105 (1907). - Z. a. a., id.: A. Seitz, Großschmett. Erde, v. 9, p. 437 (1911). $Z$. aurelia $a$., forma principalis, H. Stichel, Tierreich, Lief. 34, p. 72 (1912). Z. aurelius a., Eecke, Zool. Mededeel. Leiden, v. 4, p. 84 (1918). - W. Rothschild, Journ. Feder. Malay Stat. Mus., v. 8, p. 153 (1920). - Z. a., Zeunidia (laps.!) a., W. H. Evans, Journ. Bombay Nat. Hist. Soc., v. 29, p. 796, 797 (1923). - Zeuxidia a. a., Pendlebury, Journ. Feder. Malay Stat. Mus., v. 11, p. 34 (1923). - Z, a., W. H. Evans, Identif. Ind. Butterfl., p. 87 (1927). - Z. a. a., Godfrey, Journ. Siam Soc. Nat. Hist., Suppl., v. 7, p. 261 (1930).

subsp. a u reliana (Honr.) Amaxidia (Zeu- Südxidia) aurelius var. aureliana, Honrath, Ber- Borneo lin. Ent. Zeit., v. 33, p. 162 (1889). - $A$. aureliana, F. Moore, Lep. Ind., v. 2, p. 178 (1895). - A. aurelius aureliana, Fruhstorfer, Berlin. Ent. Zeit., v. 49, p. 190 (1904). A. aureliana, Shelford, Journ. Straits Branch Asiat. Soc., Nr. 41, p. 107 (? part.) (1904). - Zeuxidia aurelia aureliana, H. Stichel, Gen. Ins., fasc. 36, p.: 23 (1906). - Z. aurelius aureliana, Fruhstorfer: A. Seitz, Großschmett. Erde, v. 9, p. 437, t. 103 b $\left(\sigma^{\top}\right.$, ) (1911). - Z a arelia aureliana forma principalis, H. Stichel, Tierreich, Lief. 34, p. 71,72 , f. 9 (O) (1912). - Z. aurelius aureliana, Moulton, Journ. Straits Branch Asiat. Soc., Nr. 65, p. 16 (? part.) (1913). $Z$. aurelius var. aureliana, N. D. Riley, Cat. Type Spec. Rhop. Brit. Mus., pars 1, p. 9 (1924). Z. a $u \operatorname{relius}$, Hewitson, Exot. Butt., v. 3 (4), t. [53] Zeuxidia \& Aemona, f. 1, 2 (1868). - ? Herb. Druce, Proc. Zool. Soc. London, p. 341 (1873). - Amaxidia (Z.) a., Honrath, Berlin. Ent. Zcit., v. 31, p. 352 (O) (part.) (1887). - A. a., F. Moore, Lep. Ind., v. 2, p. 178 (in cit.) (1895).

subsp. euthycrite Fruhst. Amaxidia nov. Nordsubsp., Fruhstorfer, Berlin. Ent. Zeit., v. 49, Borneo p. 190 (1904). - Zeuxidia aurelius e., id.: A.

Seitz, Großschmett. Erde, v. 9, p. 437 (1911).

- Z. aurelia aureliana forma e., H. Stichel, Tierreich, Lief. 34, p. 73 (1912).

? A maxidia a ureliana, Shelford, Journ. Straits Branch Asiat. Soc., Nr. 41, p. 107 (1904). - ? Zeuxidia aurelius aureliana, 


\section{Genus Thauria F. Moore}

F. Moore, Lep. Ind., v. 2, p. 173, 185 (Typ. T. aliris) (1895). Crowley, Ann. Mag. Nat. Hist., ser. 6, v. 17, p. 66 (1896). H. Stichel, Deutsch. Ent. Zeit. Iris, v. 15, p. 59 (1902). - Fruhstorfer, Ins.-Börse, v. 20, p. 396 (1903). - T. (Sectio), Shelford, Journ. Straits Branch Asiat. Soc., Nr. 41, p. 108 (1904). T., Bingham, Taun. Brit. Ind., Lep., v. 1, p. 195 (1905). - H. Stichel, Gen. Ins., fasc. 36, p. 24, t. 1, f. 5 (Morphol.) (1906). Thauris (!), A. Pagenstecher, Geogr. Verbr. Schmett., p. 413 (1909). - Thauria, Fruhstorfer: A. Seitz, GroBschmett. Erde, v. 9, p. 441 (1911). - H. Stichel, Tierreich, Lief. 34, p. 5, 74; f. 10 (Morphol.) (1912). - W. H. Evans, Journ. Bomb. Soc. Nat. Hist., v. 21, p. 571 (1912). - Moulton, Journ. Straits Branch Asiat. Soc., Nr. 65, p. 16 (1913). - Thauria (Thaumantis), W. Rothschild, Nov. Zool,, v. 23, p. 309 (1916). - W. H. Evans, Joum. Bombay Nat. Hist. Soc., v. 29, p. 793, 796, t. 16, $\mathrm{E} 6$ (1923). - Id., Identif. Ind. Butterfl., p. 85, 86, t. 16, E 6 (1927).

Thaumantis (part.), Westwood, Trans. Ent. Soc. Iondon, n. ser., v. 4, p. 170 (1858). - Herrich-Sehäffer, Corr.Bl. Zool.-Min. Ver. Regensb., v. 19, p. 88 (1865). - W. F. Kirby, Cat. Diurn. Lep., p. 120 ; Suppl., p. 846 (1871 \& 77). - Thaumantias (!), Id,, Entomologist, v. 10, p. 292 (1877). $\left.-T .^{*}\right)$, A. G. Butler, Journ. Linn. Soc. London, Zool., v. 13, p. 115, 196 (1878). - T. (part.), id., Trans. Ent. Soc. London, ser. 2, Zool., v. 1, p. 538 (1879). - Id., Ann. Mag. Nat. Hist, ser. 5, v. 10, p. 372 (1882). - Distant, Rhop. Malay., p. 79 (1882). - G. F. L. Marshall \& Nicéville, Butt. Ind. Burm. Ceyl., v. 1. II, p. 307 (1883). O. Staudinger (\& Schatz), Exot. Schmett., v. 1, p. 203 (1887). - E. Haase, Corr.-Bl. Ent. Ver. Iris, v. 1, p. 306 (Organ. ador.) (1888). - Elwes (\& O. Möller), Trans. Ent. Soc. Iondon, p. 282 (1888). - Röber: O. Staudinger \& Schatz, Exot. Schmett., v. 2, p. 185 (1889). - Elwes, Proc. Zool. Soc. Liondon, p. 271 (1891). - Eimer (\& Fickert), Entstehg. d. Arten, pars 2, Orthogen., p. 236 (1897). - E. Y. Watson, Jonrn. Bombay Nat. Hist. Soc., v. 10, p. 652 (1897). - Hartert, Nov. Zool., v. 9, p. 232 (1902). - Moulton, Entomologist, v. 45, p. 247 (1912). Id., Journ. Straits Branch Asiat. Soc., Nr. 63, p. 79 (1912). - Taumantis (!), Moulton, Entomologist, v. 45, p. 247 (1912). - Thaumantis, N. D. Riley \& Gabriel, Cat. Type Spec. Rhop. Brit. Mus., pars 1, p. 30 etc. (1924).

M o $r$ p hindra, Röber, Ent. Zeit. Stettin, v. 64, p. 337 (Typ. M. aliris) (1903).

aliris (Westw.) Thaumantis a., Westwood, Trans. Borneo, Ent. Soc. London, sor, 2, v. 4, p. 176 (part.) Malayische (1858). - Thauria a., H. Stichel, Gen. Ins., Halbinsel, fasc. 36, p. 25 (part.) (1906). - Fruhstor- Tenasserim, fer: A. Seitz, GroBschmett. Erde, v. 9, p. Burma 441 (part.) (1911). - H. Stichel, Tierreich, Lief. 34, p. 76 (part.) (1912). - W. Pothschild, Nov. Zool., v. 23, p. 309 (1916).

- subsp. aliris (Westw.) Thaumantis a., West- Borneo wood, ut antea $\left(Q\right.$, non $\sigma^{\top}:=T$. a. pseudaliris) (1858). - Herrich-Schäffer, Corr.-

*) vide Corrigenda et Addenda pag. 162. 
Bl. Zool.-Min. Ver. Regensb., v. 19, p. 88 (1865). - W. F. Kirby, Cat. Diurn. Lep., p. 120 (1871). - Thaumantias a., id., Entomologist, v. 10, p. 293 (1877). - Thaumantis a., G. F. L. Marshall \& Nicéville, Butt. Ind. Burm. Ceyl., v. 1. II, p. 308 (nota) (1883). - O. Staudinger (\& Schatz), Fxot. Schmett., v. 1 t. 64, p. $204(1886 \& 87)$. - E. Haase, Corr.-Bl. Fnt. Ver. Iris, v. 1, p. 307 (Organ. odor.) (1888). - Thauria a., F. M[oore, Lep. Ind., v. 2, p. 187 (1895). - Thaumantis a., Fimer (\& Fickert), Entstelg. d. Arten, pars 2, Orthogen., p. 236 (1897). - Morphindra a., Röber, Int. Zeit. Stettin, v. 64, p. 337 (1903). - Thauria a. a., Fruhstorfer, Ins.Börse, v. 20, p. 396 (1903). - Thaumantis a., Journ. Straits Brancl Asiat. Soc., Nr. 41, p. 108 (1904). - Thauria a. a., H. Stichel, Gen. Ins., fasc. 36, p. 25 (1906). - Fruhstorfer: A. Seitz, Großschmett. Firde, v. 9, p. 441 (1911). - Taumantis (!) a., Moulton, Entomologist, v. 45, p. 247 (1912). - Thaumantis a., id., Journ. Straits Branch Asiat. Soc., Nr. 63, p. 79 (1912). - Thauria a., id., 1. c., Nr. 65, p. 16 (1913). - T. a. a., W. Rothschild, Nov. Zool., v. 23, p. 309 (1916).

subsp. pse udaliris (A. G. Butl.) Thaumatis $p$., A. G. Butler, Journ. Linn. Soc. London, Zool., v. 13, p. 115, 196 (1877 \& 78). W. F. Kirby, Cat. Diurn. Lep. Suppl., p. 846 (1877). - A. G. Butler, Trans. Linn. Soc. London, ser. 2, Zool., v. 1, p. 538, t. 68, f. 1 (1879). - Distant, Rhop. Malay., p. 79, t. 8, f. 3 (1882). - G. F. L. Marshall \& Nicéville, Butt. Ind. Burm. Ceyl., v, 1. II, p. 307, 308 (1883), - O. Staudinger (\& Schatz), Exot. Schmett., v. 1, p. 204 (1887). - E. Haase, Corr.-BI. Ent. Ver. Iris, v. 1, p. 307 (Organ. odor.) (1888). - Elwes (\& O. Möller), Trans. Ent. Soc. London, p. 282 (1888). - Flwes, Proc. Zool. Soc. London, p. 271 (1891). - Thauria p., F. Moore, Lep. Ind., v. 2, p. 185 , t. 149, f. 1, la, b (1895). - Thaumantis p., E. Y. Watson, Journ. Bombay Nat. Hist. Soc., v. 10, p. 652 (1897). - Hartert, Nov. Zool., v. 9, p. 232 (1902). - Thauria aliris p., Fruhstorfer, Ins.-Börse, v. 20, p. 396 (1903). H. Stichel, Gen. Ins., fasc. 36, p. 25 (1906). - Fruhstorfer: A. Seitz, Großschmett. Erde, v. 9, p. 441 (1911). - H. Stichel, Tierreich, Lief. 34, p. 77 (1912). - T. p., W. H. Evans, Journ. Bombay Nat. Hist. Soc., v. 21, p. 571 (1912). - T. aliris p., W. Rothschild, Nov. Zool., v. 23, p. 309 (1916). W. H. Evans, Journ. Bombay Nat. Hist. Soc., v. 27 , p. 90 (1920). - Id., l. c., v. 29 , p. 796 (1923). - Thaumantis p., N. D.

Malayische Falbinsel, Tenasserim, Süd-,

Mittel-

Burma 
Riley, Cat. Type Spec. Brit. Mus., pars 1, p. 49 (1924). - Thauria aliris p., W. H. Evans, Identif. Ind. Butterfl., p. 86 (1927).

? T. aliris intermedia (non T. $i$. Crowley), E. V. Ellis, Journ. Bomhay Nat. Hist. Soc., v. 25, p. 107 (1917). - Ollenhach, l. c., v. 27, p. 887 (1921).

suhsp. intermedia Crowley T. i., Crowley, Ann. Mag. Nat. Hist., ser, 6, v. 17, p. 66 (? part.) (1896). - T. aliris i., Fruhstorfer, Ins.-Börse, v. 20, p. 396 (1903). - H. Stichel, Gen. Ins., fasc. 36, p. 25 (1906). Fruhstorfer: A. Seitz, Großschmett. Erde, v. 9, p. 441 (1911). - H. Stichel, Tierreich, Lief. 34, p. 77,78 (1912). - T. a. „race" i., W. H. Evans, Journ. Bomhay Nat. Hist. Soc., v. 21, p. 571 (1912). T. a. p., Tytier, 1. c., v. 23, p. 227 (1914). - W. Rothschild, Ann. Mag. Nat. Hist., ser. 8 , v. 17, p. 476 (1916). - Id., Nov. Zool., v. 23 , p. 309 (1916). - T. lathyi i., W. H. Evans, Joum. Bombay "Nat. Hist. Soc., v. 27 , p. 90 (1920). - T. aliris i., id., 1. c., v. 29 , p. 796 (1923). - T. i., N. D. Riley, Cat. Type Spec. Rhop. Brit. Mus., pars 1, p. 27 (1924). - T. aliris i., W. H. Evans, Identif. Ind. Butterfl., p. 86 (1927).

Godfrey, Journ. Siam Nat. Hist. Soc., v. 7, p. $262(1930)$.

Lathyi (Fruhst.) Thaumatis aliris l., Fruhstorfer, Tonkin,

Deutsch. Ent. Zeit. Lep., v. 15, p. 177 (1902). Siam, - Thauria l., W. Rothsshild, Ann. Mag. Nat. Hist., ser. 8, v. 17, p. 475 (1916). -. Id., Nov. Zool., v. 23, p. 309 (1916).

T. a liris (non Thaumantis a. Westwood) (part.), H. Stichel, Gen. Ins., fasc. 36, p. 25 (1906). - Fruhstorfer: A. Seitz, Großschmett. Erde, v. 9, p. 441 (1911). - H. Stichel, Tierreich, Lief. 34, p. 76 (1912).

subsp. Lathyi (Fruhst.) Thaumantis ali- Tonkin ris l., Frulistorfer, ut antea $(1902)$. Id., Ins.-Börse, v. 2J, p. 393 (1903). - H. Stichel, Gen. Ins., fasc. 36, p. 25 (1906). Fruhstorfer: A. Seitz, Großschmett. Erde, v. 9 , p. 441, t. 102 b (o non $O$, cont. p. 1135) (1911). - H. Stichel, Tierreich, Lief. 34, p. 77, $7 \mathrm{~S}$ (1912). - T. lathyi l., W. Rothschild, Nor. Zool., v. 23, p. 309 (1916). T. l., W. H. Evans, Journ. Bombty Nat. Hist. Soc., v. 27 , p. 90 (1920). - Thaumantis aliris l., N. D. Riley \& Gabriel, Cal. Type Spec. Rhop. Brit. Mus., pars 1, p. 30 (1924).

subsp. siamensis W. Rothsch. T. l. s., Siam

W. Rothschild, Ann. Mag. Nat. Mist., ser. 8, v. 17, p. 475 (1916). - W. Rothschild \& Godfrey, Journ. Nat. Hist. Soc. Siam, v. 2, p. 75 (not. biol.) (1916). - W. Rothschild, Nov. Zool., v. 23, p. 309 (1916). - Godfrey, 
Journ. Nat. Hist. Soc. Siam, v. 2, p. 124 (1916). - N. D. Riley \& Gabriel, Cat. Type Spec. Rhop. Brit. Mus., pars 1, p. 53 (1924). - Godfrey, Journ. Siam Soc. Nat. Hist. Suppl., v. 7, p. 262 (1930).

subsp. a mplif a scia W. Rothsch. T. l. a., Burma,

W. Rothschild, Ann. Mag. Nat. Hist., ser. Shan

8, v. 17, p. 475 (1916). - Id., Nov. Zool., v. 23 , p. 309 (1916). - W. H. Evans, Journ. Bombay Nat. Hist. Soc., v. 27, p. $90(1920)$.

- Id., 1. c., v. 29 , p. 796 , t. 16, fig. E 6 ;

1 (1923). - Id., Identif. Butterfl., p. 86,

Staaten,

Tenasserim,

Malayische

Halbinsel

2

t. 16, fig. E 6. 1 (1927).

\section{Tribus Faunidi}

T' a e na ridi (part.), H. Stichel, Gen. Ins., fasc. 36, p. 26 (1906). - Id., Tierreich, Lief. 34, p. 79 (1912).

\section{Genus Stichophthalma C. \& R. Felder}

C. \& R. Felder, Wien. Ent. Monatschr., v. 6, p. 27 (1862). - S. H. Scudder, Proc. Amer. Ac. Sci,, v. 10, p. 271 (Typ. S. howqua) (1875). - G. F. L. Marshall \& Nicéville, Butt. Ind. Burm. Ceyl., v. 1. II, p. 283, 308 (1883). - Wood-Mason \& Nicéville, Journ. Asiat. Soc. Bongal, v. 55. II, p. 353 (1887). - Elwes \& Nicéville, 1. c., p. 419 (1887). - E. Haase, Corr.-Bl. Ent. Ver. Iris, v. 1, p. 306 (Organ. odor.) (1888). - Stictopthalma (!), Stictophthalmia, Elwes (\& O. Möller), Trans. Ent. Soc. London, p. 282, 333; corr. p. VIII (1888). - Stichophthalma, Röber: O. Staudinger \& Schatz, Txot. Schmett., v. 2, t. 30 (MForphol.); p. 184 (1888 \& 89). - Loech, Butt. Chin. Jap. Corea, จ. 1, p. 113 (1892). - S. H. Scudder, Life Butterfl., p. 104 (1893). - W. F. Kirby, Handb. Lep. (Allens Natural. Libr.) v. 1, p. 197 (1894). - Nicéville, Journ. Asiat. Soc. Bengal, v. 63. II, p. 3 (nota) (1894). - F. Moore, Iop. Ind., v. 2, p. 202 (1895). - Röber, Ent. Nachr., v. 26, p. 204 (1900). - H. Stichel, Deutsch. Ent. Zeit. Iris, V. 15, p. 59 (1902). - Röber, Ent. Zeit. Stettin, v. 64, p. 338 (1903). - Stichophthalmia (!), I. C. H. Young, Journ. Bombay Nat. Hist. Soc., v. 15, 1903, p. 297, 485 (1903 \& 04). - Stichophthalma, Bingham, Faun. Brit. Ind., Lep., v. 1, p. 182, 191 (Typ. .S. howqua) (1905). - H. Stichel, Gen. Ins., fasc. 36, p. 26, t. 1,. f. 6 (Morphol.) (1906). - Id.: A. Seitz, Großschmett. Irde, v. 1, p. 156 (1908). - Stichophtalma (!), A. Pagenstecher, Geogr. Verbr. Schnett., p. 412 (1909). - Stichophthalma, Fruhstorfer: A. Seitz, Großschmett. Erde, v. 9, p. 424 (1911). - H. Stichel, Tierreich, Lief. 34, p. 4, 79, fig. 11, 12 (Morphol.) (1912). - Stictopthalma (!), W. H. Evans, Journ. Bombay Nat. Hist., v. 21, p. 571 (1912). - Stichophthalma, W. Rothschild, Nov. Zool., v. 23, p. 307 (1916). - Stichopthalma (!), Godfrey, Journ. Nat. Hist. Soc. Siam, v. 2, p. 124 (1916). - Ollenbach, Journ. Bombay Nat. Hist. Soc., v. 26, p. 867 (1918). - Stictopthalma (!), W. H. Evans, 1. c., v. 27, p. 89, 90 (1920). Stichopthalma (!), Ollenbach, 1. c., v. 27, p. $887^{\circ}(1921)$. - Talbot, Proc. Ent. Soc. London, 1920, p. LXXXIV (1921). - Stictopthalma (!), W. H. Evans, Journ. Bombay Nat. Hist. Soc., v. 29, p. 793,794 , t. 15, E 4 (1923). - Stichophthalmu, Antram, Butterf. Ind., p. 118 (1924). - Stichophtalma (!), Le Cerf, Enc. Ent., ser. B. 3, Lep., v. 1, p. 141 (Morphol.) (1926). - Sticopthalma (!), W. II. Evans, Identif. Ind. Butterfl., p. 84, 85, t. 15, F 4 (1927). 
Tha $u$ mant is Subgen., Westwood, Cab. Orient. Entom., p. 8 (1848). - T. (part.) E. Doubleday (\& Westwood), Gen. Diurn. Irep., t. 55 (1849); Westwood, l. c., v. 2, p. 335, 533 (1851). - Id., Trans. Ent. Soc. London, n. ser., v. 1, p. 174 (1851). - Chenu \& H. Lucas, Enc. Hist. Nat., Papill., p. 166 (1851). - Horsfield \& F. Moore, Cat. Lep. Mus. E.-Ind. Comp., v. 1, p. 214 (1858), - T. (Divisio 2) Westwood, Traus, Ent. Soc. London, n. ser., v. 4, p. 170 (1858). - T. (part.) Hewitson, Exot. Butterfl., v. 4 (3), t. [3] Dasyophthalma \& Thaumantis (1862). - HerrichSchäffer, Corr.-Bl. Zool.-Min. Ver. Regensb., v. 18, p. 105; v. 19, p. 88 (1864 \& 65). - F. Moore, Proc. Zool. Soc. London, p. 761 (1865). - W. F. Kirby, Cat. Diurn. Lep., p. 119 (1871). - Thaumantias (!), (part.), id., Entomologist, v. 10, p. 292 (1877). - Thaumantis (p.), F. Moore. Proc. Zool. Soc. Loudon, p. 827 (1878). - T., Wood-Mason, Proc. Asiat. Soc. Bengal, v. 47. II, p. 175 (1878). - A. G. Butler, Trans. Ent. Soc. London, p. 2 (1879). - T. (part.), W. F. Kirby, Sci. Proc. R. Dublin Soc., ser. 2, v. 2, p. 300 (1880). - T., G. F. I. Marshall, Journ. Asiat. Soc. Bengal, v. 51. II, p. 39 (1882). - A. G. Butler, Ann. Mag. Nat. Hist., ser. 5, v. 16, p. 303 (1885). - T. (part.), IJ. Haase, Corr.-Bl. Ent. Ver. Iris, v. 1, p. 104 (1886). - O. Staudinger (\& Schatz), Fxot. Sclimett., v. 1, p. 204 (1887). T., E. Hofmann, Jahresheft Ver, Naturk. Württemb. 46, p. 236 (1890). - Robbe, Ann. Soc. Ent. Belg., v. 36, p. 128 (1892). - T. (part.), Eimer (\& Fickert), Entstehg. d. Arten, pars 2, Orthogen., p. 236 etc. (1897). - N. D. Riley \& Gabriel, Cat. Type Spec. Rhop. Brit. Mus., pars 1, p. 12 etc. (1924).

\section{Cohors a. Camadeviformes}

H. Stichel, Gen. Ins., fasc. 36, p. 27 (1906). - Id., Tierreich, Lief. 34, p. 81 (1912).

camadera (Westw.) Morpho (Thaumantis) c., West- Nordwood, Cab. Orient. Entom., p. 9 (part.) Indien: (1848). - Stichophthalma c., H. Stichel, SikkimGen. Ins., fasc. 36, p. 27 (1906). - Fruh- Burma storfer: A. Seitz, Großschmett. Erde, v. 9, p. 425 (1911). - H. Stichel, Tierreich, Lief. 34, p. 81 (1912). - IV. H. Evans, Journ. Bombay Nat. Hist. Soc., v. 23, p. 301 (synop. sec. Fruhstorfer) (1914).

subsp. camadeva (Westw.) Morpho (Thau- Sikkim mantis) c., Westwood, ut antea, t. 4, f. 1, 2 (1848). - Th. c., (E. Doubleday \&) Westwood, Gen. Diurn. Lep., t. 55, f. 2 ; v. 2, p. 335 (1849 \& 51). - Chenu \& H. Lucas, Enc. Hist. Nat., Papill., p. 166, t. 31 (1851). Horsfield \& F. Moore, Cat. Lep. Mus. E.-Ind. Comp., v. 1, p. 216 (1857). - Westwood, Trans. Ent. Soc. London, ser. 2, v. 4, p. 177 (1858). - W. F. Kirby, Cat. Diurn. Iep., p. 120 (1871). - Id., Entomologist, v. 10 , p. 293 (1877). - Stichophthalma c., G. F. L. Marshall \& Nicéville, Butt. Ind. 
Burm. Ceyl., v. 1. II, p. 309 (part.), fig. 310 (1883). - Nicéville, Journ. Asiat. Soc. Bengal, v. 54. II, p. 2 (1885). - Thaumantis c., O. Staudinger (\& Schatz), Exot. Schmett., v. 1, t. 65 ; p. 204 (1886 \& 87). - E. Haase, Corr.-Bl. Ent. Ver. Iris, v. 1, p. 104, 306 (Organ. odor.) (1886 \& 88). Stictopthalma c., Stictophthalmia c., Elwes \& (O. Möller), Truus. Ent. Soc. London, p. 282, 333; corr. p. VIII (1888). - Stichophthalma c.. W. F. Kirby, Haudb. Lep. (Allens Natural. Libr.), v. 1, p. 197 (1894). - Nicéville, Gazettecr Sikhim, List Butterfl., p. 131 (1594). - F. Moore, Lep. Ind., v. 2, p. 203 (part.), t. 158 , f. 1 , 1 a $(1895)$. Thaumantis c., Himer (\& Fickert), Entstehg. d. Arten, pal's 2, Orthogen., p. 236 (1897). - Stichophthalmia c., L. C. H. Young, Journ. Bombay Nat. Hist. Soc., v. 15, 1903, p. 485 (1904). - Stichophthalma c.s Bingham, Faun. Brit., Id., Isep., v. 1, p. 192 (part.) (1905). - S. c. c., H. Stichel, Gen. Ins., fasc. 36, p. 27 (cit. part.) (1906). Fruhstorfer: A. Seitz, Großsclimett. Erde, v. 9, p. 426 (1911). - H. Stichel, Tierreich, Lief. 34, p. 83 (part.) (1912). Stictopthalma c., W. H. Evans, Journ. Bombay Nat. Hist. Soc., v. 21, p. 571 (1912). S. c. c., id., 1. c., v. 29, p. 795 (1933). Stichophthalma c., Antrum, Butt. Ind., p. 118 (part.), fig. 246 (1924). - M. Hering, Biol. Schmett., p. 154 (odor) (1926). Sticopthalma c. c., W. H. Evans, Identif. Ind. Butterfl., p. 85 (1927).

subsp. Nicévillei Köb. S. c. var. n., Assam,

Röber, Ent. Nachr., v. 26, p. 203 (1900). - ? Bengalen

S. c. n., Frulistorfer: A. Seitz, Großschmett. Erde, v. 9, p. 426 (1911). - Stictopthalma c. ,1ace" n., W. H. Evans, Journ. Bombay Nat. Hișt. Soc., v. 21, p. 571 (1912). S. c. n., id., 1. c., v. 29, p. 795 (1923). Id., Identif. Ind. Butterfl., p. 85 (1927).

? Thaumantis camadeva, F. Moore, Proc. Zool. Soc. London, p. 761 (1865). T. c., A. G. Butler, Trans. Ent. Soc. London, p. 2 (1879), - Stichophthalma c., G. F. L. Marshall \& Nicéville, Butt. Ind. Burm. Ceyl., p. 309 (part.) (1883). - Thaumantis c., A. G. Butler, Ann. Mag. Nat. Hist., ser. 5, v. 16, p. 303 (1885). - Stichophthalma c., Nood-Mason \& Nicéville, Journ. Asiat. Soc. Bengal, v. 55. II, p. 353 (1887). - ? Thaumantis c., Robbe, Ann. Soc. Ent. Belgique, v. 36, p. 128 (1892). - Stichophthalma c., F. Moore, Lep. Ind., v. 2, p. 303 (part.) (1895). - Bingham, Faun. Brit. Ind., Lep., v. 1, p. 192 (1905). - S. camadeva camadevoides (non Nicéville), H. Stichel, Gen. Ins., fasc. 36, p. 27 (in cit.) (1906). - 
Id., Tierreich, Lief. 34, p. 83 (in cit.) fig. 13 (ㅇ) (1912). - S. camadeva, Antram, Butterfl. Ind., p. 118 (part.) (1924).

forma nagaens is W. Rothsch. S. c. n., Typ.:

W. Rothschild, Nov. Zool., v. 23, p. 308 (1916). - Stictopthalma c. n., W. H. Evans, Journ. Bombay Nat. Hist. Soc., v. 27, p. 90 (1920). - Id., l. c., v. 29, p. 795 (1923). - Id., Identif. Ind. Butterfl., p. 85 (1927). subsp. c a madevoides Nicév. S. c., Nicéville, Journ. Bombay Nat. Hist. Soc., v. 12, p. 330 (1898). - S. camadeva camadevoides, H. Stichel, Gen. Ins., fasc. 36, p. 27 (cit. part.) (1906). - Fruhstorfer: A. Seitz, GroBschmett. Erde, v. 9, p. 426, t. $103 \mathrm{~b}$ ( $\left.{ }^{7}\right)$ (1911). - H. Stichel, Tierreich, Lief. 34, p. 82, 83 (cit. part.) (non fig. 13: = S. c. nicévillei) (1912). - Stictopthalma camadeva "race" camadevoides, W. H. Evans, Journ. Bombay Nat. Hist. Soc., v. 21, p. 571 (1912). - S. camadeva camadevoides, id., l. c., v. 29 , p. 795 (1923). - Sticopthalma c. c., id, Identif. Ind. Butterfl., p. 85 (1927). - Stichophthalma c. c., Godfrey, Journ. Siam Nat. Hist. Soc. Suppl., v. 7, p. 258 (1930).

cambodia (Hew.) Thaumantis c., Hewitson, Exot. Butt., จ. 4 (3) Dasyophthalma \& Thaumantis (1862). - Stichophthalma c., W. Rothschild, Nov. Zool., v. 23, p. 308 (1916).

subsp. c a mbodia (How.) Thaumantis c., Hewitson, ut antea, fig. 2 (1862). - Herrich-Schäffer, Corr.-BI. Zool.-Min. Ver. Regensb., v. 19, p. 88 (1865). - W. F. Kirby, Cat. Diurn. Lep., p. 120 (1871). - O. Staudinger (\& Schatz), Exot. Schmett., v. 1. p. 204 (1887). - Stichophthalma c., F. Moore, Lrop. Ind., v. 2, p. 207 (1895). - H. Stichel, Gen. Ins., fasc. 34, p. 27 (1906). - Fruhstorfer: A. Seitz, GroBschmett. Erde, v. 9, p. 425 (1911). - H. Stichel, Tierreich, Lief. 34 , p. 81,84 (1912). - Thaumantis c., N. D. Riley \& Gabriel, Cat. Type Spec. Rhop. Brit. Mus., pars 1, p. 12 (1924).

subsp. editha Riley \& Godfr. S. c. e., N. Siam

D. Riley \& Godfrey, Journ. Nat. Hist. Soc.

Siam, v. 4, p. 173, t. 4 , f. 5 (1921). $-\mathrm{N}$. D. Riley \& Gabriel, Cat. Typo Spec. Rhop. Brit. Mus., pars 1, p. 19 (1924). - Godfrey, Journ. Siam Soc. Nat. Hist., Suppl., v. 7, p. 259 (1930).

Godfreyi W. Rothsch. (? subsp. praeced.) S. g., W. Siam, Rothschild, Ann. Mag. Nat. Hist., ser. 8, v. Burma, 17, p. $474(1916)$. - W. Rothschild \& God- Tenasserim frey, Journ. Nat. Hist. Soc. Siam, v. 2, p. 7 (not. biol.) (1916). - W. Rothschild, Nov. Zool., v. 23, p. 308 (1916). - Stichopthal- 
ma g., Godfrey, Journ. Nat. Hist. Soc. Siam, v. 2, p. 124 (1916). - Ollenbach, Journ. Bombay Nat. Hist. Soc., v. 26, p. 867,868 , fig. (1918). - Stictopthalma g., W. II. Evans (sec. Rothsehild), 1. c., v. 27, p. 90 (1920). - Ollenbach, 1. c., v. 27, p. 887 (1921). - W. H. Evans, 1. c., v. 29, p. 795 (1923). - Stichophthalma g., N. D. Riley, Cat. Type Spec. Rhop. Brit. MLus., pars 1, p. 23 (1924). - Sticopthalma g., W. H. Evans, Identif. Ind. Butterfl., p. 86 (1927).

Fruhstorferi Röb. S. f., Röber, Soc. Ent., v. 17, p. Tonkin

153 (1903). - S. loursa f., H. Stichel, Gen. Ins., fasc. 34, p. 28 (1906). - Fruhstorfer: A. Seitz, Großschmett. Erde. v. 9, p. 425 , t. 103 a (1911). - H. Stichel, Tierreich, Iief. 34, p. 85,86 (1912). - S. f., W. Pothschild, Nov. Zool., v. 23, p. 339 (spec. distincta!) (1916). - Stictopthalma lauisa $f$. W. H. Evans, Journ. Bombay Nat. Hist. Soc., \%. 29, p. 795 (? part.) (1923). Sticophthalma i. ., id., Identif. Butterfl., p. 86 (1927).

Louisa (Wool.-Mas.) Thammant's l, Wood-Mason, Burma, Proc. Asiat. Soc. Benoal, p. 163 (part.) Siam, (1877). - Stichophthelmi $l$. (nart.). H. ?Perak Stichel, Gen. Ins., fasc. 36, p. 23 (1906). Fruhstorfer: A. Seitz, Großschmett. Erde, v. 9 , p. 425 (1911). - H. Stichel, Tierreich, Lief. 34, p. 81,84 (1912).

- subsp. Louisa (Wood.-Mrs.) Thaumantis l., Tenasserim Wood-Wason, ut autea $\left(\sigma^{\prime}\right)(1877)$. — Id., Journ. Asiat. Soc. Bengal, v. 47. II, p. 175 , t. 12 (1878). - F. Moore, Proc. Zool. Soc., p. 827 (1878). - G. F. L. Marshall, Journ. Asiat. Soc. Bengal, v. 51. II, p. 39 (Q) (1882). - Stichophthalma l., G. F. I. Marshall \& Nicéville, Butt. Ind. Burm. Ceyl., v. 1. II, p. 311 (1883). - Thaumantis l., O. Staudinger (\& Schatz), Exot. Schmett., v. 1, p. 204 (? S. howqua forma loci) (1887). - Stichophthalma l., F. Moone, Lep. Ind., v. 2 , p. 204 , t. 159 , f. 1,1 a $\left(\sigma^{\prime}\right.$,, ) $(1895)$. Bingham, Faun. Brit. Ind., Lep., v. 1, p. 194 (part.) (1905). - S. l. l., H. Stichel, Gen. Ins., fasc. 36, p. 23 (part.) (1906). Fruhstorfer: A. Seitz, Großschmett. Erde, v. 9 , p. 425 (1911). - H. Stichel, Tierreich, Lief. 34, p. 85 (1912). - Stictopthalma l. l. W. H. Evans, Journ. Bombay Nat. Hist. Soc., v. 29, p. 795 (1923). - Stichophthalma $7 .$, Röber, Ent. Rundschau, v. 43, p. 19, fig. (1926). - Sticophthalme l. l., W. H. Evans, Identif. Ind. Butterfl., p. 86 (1927). - Stichophthalma l. l., A. F. Rosa, Entomologist, v. 62, p. 226 (1929).

subsp. A $n$ to $n$ ia Röb. S. l. a., S. a., Röber, Burma

Ent. Rundschau, v. 43, p. 18, 19, fig. (1926). 
? S. lou isa, Elwes, Proc. Zool. Soc. Lond., p. 271 (1891). - Fonrath, Berlin. Ent. Zeit., v. 36, Sitzg.-Ber., p. XII (S. howqua "Iokalform") (1891). - Fruhstorfer, 1. c., v. 40. Sitzg.-Ber., p. (15) (1895). - Bingham, Faun. Brit. Ind., Lep., v. 1, p. 194 (part.) (1905). - S. l. l., H. Stichel, Gen. Ins., fasc. 36, p. 28 (part.) (1906). - Id., Tierreich, Lief. 36, p. 85 (part.) (1912). Stictopthalma l. l., W. H. Evans, Journ. Bombay Nat. Hist. Soc., v. 21, p. 57 (? part.) (1912).

subsp. siamensis W. Rothsch. S. l. s., Siam W. Rothschild, Nov. Zool,, v. 23, p. 308 (1916). - Godfrey, Journ. Siam Soc. Nat. Hist., Suppl., v. 7, p. 258 (1930).

S. lou isa, Elwes \& Nicéville, Journ. Asiat. Soc. Bengal, v. 56. II, 1886, p. 419 (1887).

subsp. Mathilda Jan. S. m., Janet, Bull, Tonkin

Soc. Ent. France, p. 215 (1905). - Stichophthalma louisa m., H. Stichel, Gen. Ins., fasc. 36 , p. 28 (1906). - Fruhstorfer: A. Seitz, Großschmett. Erde, v. 9, p. 86 (1911). - H. Stichel, Tierreich, Lief. 34, p. 85,86 (1912). - W. Rothschild, Nov. Zool., v. 23, p. 308 (1916). - S. m, Röber, Ent. Rundschau, v. 43 , p. 22 (1926).

sparta Nicév. S. 8., Nicéville, Journ. Asiat. Soc. AssamBengal, v. 63. II, p. 2 (1894). - Tytler, Burma Proc. Ent. Soc. London, v. 3. I, p. 15 (1928).

S. howqua (non Thaumantis $h$. Westwood) (part.), H. Stichel, Gen. Ins, fasc. 36, p. 28 (1906). - Fruhstorfer: A. Seitz, Großschmett. Erde, v. 9, p. 425 (1911). - H. Stichel, Tierreich, Lief. 34 , p. 86 (1912).

subsp. s p a r t a Nicév. S. s., Nicéville, ut an- Assam, tea, t. 1 , f. 4 (non 0 , sed $\sigma^{\prime \prime}$, sec. Tytler, Nord1928). - F. Moore, Lep. Ind., v. 2, p. 217, Burma t. 167 , f. 2, 2 a $\left(0^{*}\right)(1895)$. - S. howqua s., H. Stichel, Gen. Ins., fasc. 36, p. 28 (1906). - Fruhstorfer: A. Seitz, Großschmett. Erde, v. 9, p. 425 (1911). - H. Stichel, Tierreich, Lief. 34, p. 87, 88 (1912). - Stictopthalma h. "race" s., W. H. Evans, Journ. Bombay Nat. Hist. Soc., v. 21, p. 571 (1912). - S. s., Tytler, 1. C., v. 23, p. 227 (variatio) (1914). - W. Rothschild, Nov. Zool., v. 23, p. 308 (1916). - W. Rothschild (\& Godfrey), Journ. Nat. Hist. Soc. Siam, v. 2, p. 74 (1916). - Stictopthalma s., W. H. Evans (sec. Rothschild), Journ. Bombay Nat. Hist. Soc., v. 27, p. 89 (1920). - S. howqua s., id., 1. c., v. 29, p. 795 (1923). - Sticopthalma h. s., id., Identif. Butterfl., p. 86 (1927). - Stichophthalma s. s., Tytler, Proc. Ent. Soc. London, p. 16 (1928). 
S. howqua (non Thaumantis $h$. Westwood) (part.), Bingham, Fauna Brit. Ind., Lep., v. 1, p. $192(1905)$.

forma $E v a n s i$ Tytl. S. sparta e., Tytler, Typ.:

Proc. Ent. Soc. London, v. 3. I, p. 16 Nordost(1928).

Burma

subsp. Archbaldi Tytl. S. s. a., Tytler, Nord-ShanProc. Ent. Soc. London, v. 3. I, p. 15, 16 Staaten (1928).

S. lou is a fruhstorferi (non S. f. Röber), W. H. Evans, Journ. Bombay Nat. Hist. Soc., v. 21, p. 571 (? part., sec Tytler) (1912).

Tytleri W. Rothsch. (? forma praeced.) S. t., W. Assam, Rothschild, Nov. Zool., v. 25, p. 310 (1918). Nord- Stictopthalma $t$., W. H. Evans, (sec. Burma Rothschild), Journ. Bombay Nat. Hist. Soc., v. 27 , p. 89 (? = S. sparta) (1920). Id., l. c., v. 29 , p. 795 , t. 15 , fig. E 4.4 (1923). - N. D. Riley \& Gabriel, Cat. Type Spec. Rhop. Brit. Mus., pars 1, p. 59 (1924). - Sticapthalma t., W. H. Erans, Identif. Ind. Butterfi. p. 86 , t. 15 , fig. E4. 4 (1927). - S. sparta t., Tytler, Proc. Errt. Soc. London. v. 3. I, p. 16 (1928).

howqua (Westw.) Thaumantis $h$., Westwood, Trans. China, Ent. Soc. London, n. ser., v. 1, p. 174 Formosa, (1851). - Stichophthalma h. (part.), II. Stichel, Gen. Ins., fasc. 36, p. 86 (1906). Fruhstorfer: A. Soitz, Großschmett. Erde, v. 9, p. 425 (1911). - H. Sticlıel, Tierreich, Lief. 34, p. 81,86 (1912).

subsp. how qua (Westw.) Thaumantis $h$. Ost-, ZentralWestwood, ut antea (1851). - (E. Doubleday China \&) Westrood, Gen. Diurn. Lep., v. 2, p. 533 (1852). - Westwood, Trans. Ent. Soc. Lond., n. ser., v. 4 , p. 178 , t. 18 , f. 2 , 2 a $(1858)$. Stichophthalma h., C. \& R. Felder, Wien. Ent. Monatschr., v. 6, p. 27 (1862). - Thaumantis $h$., Herrich-Schäffer, Corr.-Bl. Zool.Min. Ver. Regensb., v. 19, p. 88 (1865). - W. F. Kirby, Cat. Diurn. Iep., p. 120 (1871). - Thaumantias h., id., Entomologist, v. 10, p. 293 (1877). - Stichophthalma h., G. F. I. Marshall \& Nicéville, Butt. Ind. Burm. Ceyl., v. 2, p. 311 (nota) (1883). - Thaumantis $h$. . E. Haase, Corr.-Bl. Ent. Ver. Iris, v. 1, p. 104, 306 (Organ. odor.) (1886 \& 88). - O. Staudinger (\& Schatz), Exot. Schmett., v. 1, t. 65, p. 204 (distr. part.) $(1886$ \& 87). - F. Kofmann, Jahresber. Ver. Naturk. Württemb., v. 46, p. 236 (Organ. odor.) (1890). - Stichophthalma h., Leech, Butt. Chin. Jap. Corea, p. 113 (part.) (non t. 1, f. $3:=S$. h. suffusa) (1892).W. F. Kirby, Handb. Lep. (Allens Natural. Libr.), v. 1, p. 197 (1894). - F. Moore, Lep. Ind., v. 2, p. 206 (1895). - Thau- 
mantis h., Eimer (\& Fickert), Entstehg. d. Arten, pars 2, Orthogen., p. 236, 316 (1897). - Stichophthalma h., Suffert, Ins.Börse, v. 16, p. 22 (1899). - S. h. h., H. Stichel, Gen. Ins., fasc. 36 , p. 28 (1906). S. h., id.: A. Seitz, Großschmett. Erde, v. 1, p. 156 , t. 49 a (1908). - Fruhstorfer: A. Seitz, Großschmett. Erde, v, 9, p. 425 (1911). - H. Stichel, Tierreich, Lief. 34, p. 86,87 (1912). - Stictopthalma h., W. H. Evans, Journ. Bombay Nat. Hist. Soc., v. 27, p. 89 (1920). - Tharmantis h., N. D. Riley \& Gabriel, Cat. Type Spec. Rhop. Brit. Mus., pars 1, p. 25 (1924).

subsp. mi yan a Fruhst. S. h. m., Fruhstorfer, Ent. Rundschau, v. 30, p. 133 (1913).

Süd-China (Kanton)

West-China Butt. Chin. Jap. Corea, v. 1, p. 114, t. 1, f. 3 (ㅇ) (1892). - S. s., F. Moore, Lep. Ind., v. 2, p. VIII (1896). - S. howqua var. s., Suffert, Ins.-Börse, v. 16 , p. 22 (1899). Id., Berlin. Ent. Zeit., v. 45, Sitz.-Ber., p. (2) (1900). - S. h. s., I. Stichel, Gen. Ins., fasc. 36, p. 28 (cit. part.) (1906). S. s., id.: A. Seitz, Großschmett. Erde, v. 1, p. 156 (part.), t. 49 a (1908). - Fruhstorfer: A. Seitz, Großschmett. Firde, v. 9, p. 425 (1911). - H. Stichel, Tierreich, Lief. 34, p. 87, 88 (part.) (1912). - S. h. var. s., N.D. Riley \& Gabriel, Cat. Type Spec. Rhop. Brit. Mus., pars 1, p. 55, 56 (1924).

? S. how q u a, Honrath, Berlin. Ent. Zeit., v. 36, Sitz.-Ber., p. XII (1891). - R. South, Journ. Bombay Nat. Hist. Soc., v. 22, p. 352 (transit.) (1913). - ? Draeseke, Deutsch. Ent. Zeit. Iris, v. 39, p. 57 (1925).

S. fus ca, F. Moore, Lep. Ind., v. 2, p. 206 (cor1. errata, p. VIII: suffusa) (1895 \& 96).

subsp. Bow ring $i$ Joicey \& Talb. S. h. nov. subsp., Talbot, Proc. Eut. Soc. London, 1920, p. LXXXIV (1921) - S. h. b., Joicey \& Talbot, Bull. Hill Mus., v. 1, p. 172, t. 23, f. 19 (1921). - Tid., 1. e., v. 2, p. 184 (1923).

subsp. formasana Fruhst. S. h. f., Fruhl- Formosa storfer, Entom. Wochenbl. (Insekt.-Börse), v. 25 , p. 38 (1908). - Id.: A. Seitz, Großschmett. Erde, v. 9, p. 425 , t. 103 a (1911). - H. Stichel, Tierreich, Lief. 34, p. 87,88 (1912).

- subsp. tonkiniana Fruhst. S. $h$. t.. Fruhstorfer, Soz. Entom., v. 16, p. 97 (1901). Id.: A. Seitz, Großschmett. Erde, v. 9, p. 425 , t. 103 a (aber1. O) (1911).

S. howqua $s u f f u s a$ (non Leech), H. Stichel, Gen. Ins., fasc. 36 , p. 28 (in cit.) (1906). - S. s., id.: A. Seitz, Großschmett.
Hainan

Tonkin 
Erde, v. 1, p. 156 (1908). - S. h. s., id.,

Tierreich, Lief. 34 , p. 88 (in cit.) (1912).

subsp. Wi l h e l m a Röb. S. $h$. w., Röber, Xnt. Assam

Rundschau, v. 23, p. 22 , 23, fig. $\sigma^{\prime}$, o

(1926).

? S. ho $w q u a$, Antram, Butterfl. Ind., p. 121, f. 248 (1925).

Neumogeni Leech S. n., Leech, Butt. Chin. Jap. China, Corea, v. 1, p. 114 (1892). Hainan

subsp. Neumogeni Leech $S$. n., Leech, Westut antea, t. 1 , f. 5 (1892). - F. Moore, China Lep. Ind., v. 2, p. 206 (1895). - H. Stichel, Gen. Ins., fasc. 36 , p. 28 , t. 4, f. 8 (O) (1906). - Id.: A. Seitz, Großschmett. Erde, v. 1, p. 156 , t. 49 b (1908). - Fruhstorfer: A. Seitz, Großschmett. Erde, v. 9, p. 425 (1911). - H. Stichel, Tierreich, Lief. 34, p. 81,89 (1912).

subsp. le S. n. $l$., Joicey \& Talbot, Bull. Hill Hainan MLus., v. 1, p. 173, t. 28 , f. 20 (1921). Tid., l. c., v. 2, p. 184 (1928).

Stichopthalma neumogeni, Talbot, Proc. Ent. Soc. London, 1920, p. LXXXIV (1921).

nourmahal (Westw.) Thaumantis n., (E. Double- Sikkimday \&) Westwood, Gen. Diurn. Lep., v. 2, Bhutan, p. 337 (nota) (1851). - Stichophthalma n., Hainan H. Stichel, Gen. Ins., fasc. 36, p. 29 (1906). - Fruhstorfer: A. Seitz, Großschmett. Erde, v. 9, p. 421 (1911). - H. Stichel, Tierreich, Lief. 34 , p. 81,90 (1912).

subsp. nourmahal (Westw.) Thaumantis Sikkim, n., (E. Doubleday \&) Westwood, ut an- Assam tea (1851). - Horsfield \& F. Troone, Cat. Lep. MLus. E.-Ind. Comp., v. 1, p. 215 (1857). - Westwood, Trans. Fnt. Soc. London, n. ser., v. 4, p. 178 , t. 18, f. 1,1 a (O) $(1853)$. T. nourmanhal (I), Herrich-Schäffer, Corr.Bi. Zool.-Min. Ver. Regensb., v. 19, p. 88 (1865). - T. nourmahal, W. T. Kirby, Cat. Diurn. Lep., p. 120 (1871). - Thaumantias n., id., Entomologist, v. 10, p. 293 (1877). - Stichophthalma n., G. F. L. Marshall \& Nicéville, Butt. Ind. Burm. Ceyl., v. 1. II, p. 312 (distr. part.) (1883). - Thaumantis n., 0 . Staudinger (\& Schatz), Exot. Schmett., v. 1, p. 204 (1887). - Stichophthalma n., E. Haaso, Corr.-Bl. Ent. Ver. Iris, v. 1, p. 306 (Organ. odor.) (1888). - Stictopthalma n., Stictopthalmia n., Hlwes (\& O. Möller), Trans. Ent. Soc. London, p. 282,333 ; p. VIII (1888). - Stichophthalma n., Nicéville, Gazetteer Sikhim, List Butterfl., p. 131 (1894). - F. Moone, Lep. Ind., v. 2, p. 205 , t. 160 , f. $1,1 \mathrm{a}, \mathrm{b}$ (o', ㅇ) - S. n. n., H. Stichel, Gen. Ins., fasc. 36, p. 29 (1906). - Fruhstorfer: A. Seitz, 
Großschmett. Erde, v. 9, p. 424 (1911). -

H. Stichel, Tierreich, Lief. 31, p. 92 (1912).

- Stictopthalma n., W. H. Evans, Journ. Bombay Nat. Hist. Soc., v. 21, p. 571 (1912).Stichophthalma n., Tytler, 1. c., v. 23, p. 227 (1914). - Stictopthalma n. n., W. H. Evans, 1. c., v. 29, p. 795 (1923). - Sticopthalma n. n., id., Identif. Ind. Butterfl., p. 85 (1927). - Stichophthalma n., Antram, Butt. Ind., p. 120, f. 247 (1924).

subsp. $n$ urinis a Nicév. S. n., Nicéville, Bhutan Journ. Bombay Nat. Fist. Soc., v. 5, p. 131, t. O (1890). - Id., Gazetteer Sikhim, List. Butterffl, p. 131 (1894). - F. Moore, Lep. Ind., v. 2, p. 206, t. 161, f. 1, $1 \mathrm{a}_{;}$b $\left(\sigma^{3}, Q\right)$ (1895). - S. nourmahal nurinissa, H. Stichel, Gen. Ins., fasc. 36, p. 29 (1906). - Fruhstorfer: A. Seitz, Großschmett. Erde, v. 9, p. 424 (1911). - H. Stichel, Tierreich, Lief. 34, p. 92, 93 (1912). - Stictopthalma nurinissa, W. H. Evans, Journ. Bombay Nat. Hist. Soc., v. 21, p. 571 (1912). - S. nourmahal nurinissa, id., 1. c., v. 29 , p. 796, t. 15 , f. E 4.1 (1923). - Sticopthalma n. n., id., Identif. Butterfl., p. 85, t. $15, \mathrm{f}$. E. 4. 1 (1927).

subsp. Chuni Joicoy \& Talb. S. nourmahal Hainan n. subsp. Talbot, Proc. Ent. Soc. Lond., 1920, p. LXXXIV (1921). - S. nourmahal c., Joicey \& Talbot, Bull. Hill Mus., v. 1, p. 172, 10

t. 23 , f. 18 (1921).

\section{Genus Aemona Hew.}

Hewitson, Exot. Butt., v. 3 (4), t. [53] Zeuxidia \& Aemona (1868). - W. F. Kirby, Cat. Diurn. Lep., p. 119 (1871). - S. H. Scudder, Proc. Amer. Ac. Sci., v. 10, p. 103 (Typ. A. amathusia) (1875). Wood-Mason, Journ. Asiat. Soc. Bengal, v. 49. II, p. 175 (1880). C. Crüger (sec. Kirby), Berlin. Ent. Zeit., v. 25, p. 111 (1881). G. F. L. Marshall \& Nicéville, Butt., Ind. Burm. Ceyl., v. 1. II, p. 283, 302 (1883). - B. Gerlaard, Berlin. Ent. Zeit., v. 27, p. 181 (distr. geogr.) (1833). - O. Staudinger (\& Schatz), Exot. Schmett., v. 1, p. 203 (1887). - L. Glaser, Cat. Etymol. Col. Lep., p. 273 (1887): - Röber: O. Staudinger \& Schatz, Exot. Schmett., v. 2, t. 31 (Morphol. A. leva!); p. 181, 187 (1888 \& 89). - F. Moore, Lep. Ind., v. 2, p. $212(1895)$. - H. Stichol, Deutsch. Ent. Zeit. Iris, v. 15, p. 59 (1902). - L. C. H. Young, Journ. Bombay Nat. Hist. Soc., v. 15, p. 298 (1903). - Bingham, Faun. Brit. Ind., Lep., v. 1, p. 182, 186 (Typ. A. amathusia) (1905). - H. Stichel, Gen. Ins., fasc. 36, p. 29, t. 2, f. 7 (Morphol.) (1906). - A. Pagenstecher, Geogr. Verbr. Schmett., p. 413 (1909). - Fruhstorfer: A. Seitz, Großschmett. Erde, v. 9, p. 406 (1911). - H. Stichel, Tierreich, Lief. 34, p. 5, 93, f. 14 (Morphol.) (1912). - W. H. Evans, Journ. Bombay Nat. Hist. Soc., v. 21, p. 571 (1912). - W. Rothschild, Nov. Zool., v. 23, p. 300 (1916). - W. H. Evans, Journ. Bombay Nat. Hist. Soc., v. 29, p. 793, t. 16, E 3 (1923). - Antram, Butterfl. Ind., p. 121 (1924). W. H. Evans, Identif. Butterfl., p. 84, 85, t. 16, E 3 (1927). 
Clerome (non Doubleday), Hewitson, Trans. Ent. Soc. London, ser. 3, v. 5, p. 566 (1867). - N. D. Riley \& Gabriel (sec. Hewitson), Cat. Type Spec. Rhop. Brit. Mus., pars 1, p. 7 (1924).

A moena (error typ.!), H. Stichel: A. Seitz, Großschmett. Erde, v. 1, p. 156 (1908).

amathusia (Hew.) Clerome $a$. , Hewitson, Trans. Ent. SikkimSoc. London, ser. 3, v. 5, p. 566 (1867). - Tonkin,

H. Stichel, Gen. Ins., fasc. 36, p. 30 (1906). China

- Fruhstorfer: A. Seitz, Großschmett. Erde, จ. 9, p. 407 (part.) (1911). - H. Stichel, Tierreich, Lief. 34, p. 95 (part.) (1912).

subsp. a mathusia (Hew.) Clerome a., He- Sikkim, witson, ut antea (1867). - Aemona a., id., Bhutan, Exot. Butt., v. 3 (4), t. [53] Zeuxidia \& Assam, Aemona, f. 3, 4 (q) (1868). - T. T. K. Kir- Burma by, Cat. Diurn. Lep., p. 119 (1871). Wood-Mason, Journ. Asiat. Soc. Bengal, v. 49. II, p. 176, t. 6, f. 2,2 a (non f. 3,4 sec. text.) (1880). - G. F. L. Marshall \& Nicéville, Butt. Ind. Burm. Ceyl., v. 1. II, p. 303, Titelbild, f. $2,2 a(1883)$. - O. Staudinger (\& Schatz), Exot. Schmett., v. 1, p. 203 (1887). - Doherty, Journ. Asiat. Soc. Bengal, v. 58. II, p. 124 (= pealii W.-Mason) (1889). - Swinhoe, Trans. Ent. Soc. London, p. 276 (1893). - Nicéville, Gazetteer Sikhim, List Butterfl., p. 130 (1894). - F. Moore, Lep. Ind., จ. 2, p. 212 , t. 164 , f. $1 \mathrm{a}-\mathrm{e}\left(\sigma^{\prime}\right.$, f) (1895). - Eimer (\& Fickert), Entstehg. d. Arten, pars 2, Orthogen., p. 121, 236 (1897). - Binghan, Faun. Brit. Ind., Lep., V. 1, p. 196 (cit. part.) (1905). - A. a. a., H. Stichel, Gen. Ins., fasc. 36 , p. 30 (1906). - Fruhstorfer: A. Seitz, Großschmett. Erde, v. 9, p. 407 (1911). - A. a., Tytler, Journ. Bombay Nat. Hist. Soc., v. 21, p. 54 (1911). - A. a. a. forma principalis, H. Stichel, Tierreich, Lief. 34, p. 95, 97 (cit. part.) (1912). - W. H. Evans, Journ. Bombay Nat. Hist. Soc., v. 21, p. 571 (1912). - Tytler, 1. e., v. 23; p. 228 (1914). - W. H. Evans, l. c., v. 29, p. 794 (1923). - Antram, Butterfl. Ind., p. 121 , f. 249 (1924). - Clerome a., N. D. Riley \& Gabriel, Cat. Typo Spec. Rhop. Brit. Mus., pars 1, p. 7 (1924). W. H. Bvans, Identif. Ind. Butterfl., p. 571 (1927).

subsp. Oberthüri H. Stich. A. r. o., H. WestStichel, Gen. Ins., fasc. 36 , p. 31 , t. 4 , f. 10 China (Q) (1906). - Amoena o., id.: A. Seitz, Großschmett. Erde, v. 1, p. 156, t. $49 \mathrm{c}$ (q) (1908). - Aemona amathusia o., Fruhstorfer: A. Seitz, Großschmett. Erde, v. 9, p. 407 (1911). - H. Stichel, Tierreich, Lief. 34, p. 96,97 , f. 15 ( $\left.\sigma^{7}\right)(1912)$.

subsp. tonkinensis W. Rothsch. A. a. t., Tonkin W. Rothschild, Nov. Zook., v. 23, p. 300 (1916). 
Pealii Wood-Mas. A. $p$, Wood-Mason, Pros. Asiat. Assam Sos. Bengal, p. 173 (1880). - Id., Journ. Asiat. Soc. Bengal, v. 49. II, p. 177, t. 6, f. 3, 3 a (non f. 5, 6 sec. text.) (1880). G. F. L. Marshall \& Nicéville, Butt. Ind. Burm. Ceyl., v. 1. IT, p. 303, Titelbild, f. 3, 3 a (1883). - 0 . Staudinger (\& Schatz), Exot. Schmett., v. 1, p. 203 (1887). - A. peali, F. Moore, Lep. Ind., v. 2, p. 214, t. 165 , f. 1 , 1a $\left(\sigma^{\prime}\right)$ (1895). - A. amathusia var. peali, Bingham, Faun. Brit. Ind., Lep., v. 1, p. 197 (1905). - A. pealii, H. Stichel, Gen. Ins, fasc. 36, p 30 (1906). A. a. peali, Fruhstorfer: A. Seitz, Großschmett. Erde, v. 9, p. 407 (? A. amathusia, Regenzeitform) (1911). - A. a. a., forma pealii, H. Stichel, Tierreich, Lief. 34 , p. 96, 97 (1912). - A. p., Tytler, Journ. Bombay Nat. "Hist. Soc, v. 23 , p. 228 (non $P$. amathusia forma) (1914).

A. a mathusia "wet season form", Doherty, Journ. Asiat. Soc. Bengal, v. 58. II, p. 124 (1889).

lena Atkins. A. l., Atkinson, Proc. Zool. Soc. Lon- Süd-Chinadon, p. 215 (1871).

Tenasserim

subsp. le n a Atkins. A. l., Atkinson, ut antea, t. 12, f. 1 (1871). - A. leva (!), W. F. Kirby, Cat. Diurn. Lep., Suppl., p. 714 (1877). - A. lena, F. Moore, Proc. Zool. Soc. London, p. 827 (1878). - Id.: J. Anderson, Anat. Zool. Results Exp. Yunnan, v. 1, p. 924; v. 2, t. 81 , f. 1 (1878). - WoodMason, Jonrn. Asiat. Soc. Bengal, v. 49. II, p. 177 (1880). - G. F. L. Marshall \& Nicéville, Butt. Ind. Burm. Coyl., v. 1. II, p. 302 (1883). - Elwes \& Nicéville, Journ. Asiat. Soc. Bengal, v. 55. II, 1886, p. 419 (1887). - A. leva (!), A. lena, O. Staudinger (\& Schatz), Exot. Schmett., v. 1, t. 65 ; p. 203 (1886 \& 87). - A. lena, Manders, Trans. Ent. Soc. London, p. 520 (1890). F. Moore, Lep. Ind., v. 2, p. 215, t. 165, f. 2, 2a (1895). - A. leva, Eimer (\& Fickert), Entstehg. d. Arten, pars 2, Orthgen., p. 236 (1897). - A. lena, Fruhstorfer, Ins.-Börse, v. 19, p. 379 (1902). - Id., Berlin. Ent. Zeit., v. 48, Sitz.-Ber., p. (18) (1903). - H. Stichel, Gen. Ins., fasc. 36, p. 31, t. 4, f. 10 (Q) (1906). - Fruhstorfer: A. Seitz, Großschmett. Erde, v. 9, p. 407, t. 102b (1911). - H. Stichel, Tierreich, Lief. 34, p. 95, 98 (1912). - W. H. Evans, Journ. Bombay Nat. Hist. Soc., v. 21, p. 571 (1912). - Id., l. c., v. 29, t. 16, fig. E 3. 2 (1923). - N. D. Riley \& Gabriel, Cat. Typo Spec. Rhop. Brit. Mus., pars 1, p. 31 (1924). - W. H. Evans, Identif. Ind. Butterfl., p. 85, t. 16, fig. E 3. 2 (1927). - Godfrey, Journ.
Yunnan, Bhutan, Burma,

Siam, Tenasserim 
Siam Soc. Nat. Hist., Suppl., v. 7, p. 122

(1897). - Id., 1. c., v. 7, p. 258 (1930).

subsp. H a yn e $i$ Tytl. A. l. h., Tytler, Journ. Nord-Shan-

Bombay Nat. Hist. Soc., v. 31, p. 260 , t. 3 , Staaten

3

f. 9 (1926).

\section{Genus Faunis Jac. Hübn.}

F a unis (part.), Jac. Hübner, Verz. Schmett., p. 55 [1818]. $F$., id., Samml. Exot. Schmett., v. 2, t. [83], Index $(1820-26)$. S. H. Scudder, Proc. Amer. Ac. Sci., v. 10, p. 177 (nom. praeoce., sed non) (1875). - W. F. Kirby: Hübner \& Geyer, Samml. Exot. Schmett., Neue Ausg., v. 3, t. 295 (82) (1898). - H. Stichel, Gen. Ins., fasc. 36 , p. 31, t. 2, f. 8 (Morphol.) (1906). - Id.: A. Seitz, Großschmett. Erde, v. 1, p. 156 (1908). - Fruhstorfer: A. Seitz, Großschmett. Ende, v. 9, p. 404 (1911). - H. Stichel, Tierreich, Lief. 34, p. 5, 99, f. 16 (Morphol.) (1912). - Moulton, Journ. Straits Branch Asiat. Soc., Nr. 65, p. 16 (1913). - W. II. Lvans, Journ. Asiat. Soc. Bengal, v. 22, p. 762 (1914). - Eecke, Not. Ireyden Mus., v. 36, p. 228 (1914). - W. Rothschild, Nov. Zool., v. 23, p. 299 (1916). - Eecke, Zool. Mededeel. Lciden, v. 4, p. 83 (1918). - W. H. Evans, Journ. Bombay Nat. Hist. Soc., v. 29, p. 793,794 , t. 15 , E 2 (1923). - - Id., Identif. Butterfl., p. 85, 86, t. 15, E 2 (1927). - A. Seitz, Ent. Rundschau, v. 45, p. 24 (1928).

Danaus [Subgen.] (part.), Goeze, Ent. Beytr., v. 3. I, p. 124, 212 (1779). - Gmelin-Linné, Syst. Nat., ed. 13, v. 1. V, p. $2280(1790)$.

Satyrus, „S atyre" (part.), Latreillo: Enc. Móth.y v. 9, p. 11,460 (1819 \& 24). - Salyrus (part.) (Latreille \&) J. B. Godart, l. c., p. 497 (1824). - Verloren, Cat. Ins. Lep. Cramer, v. 2, p. 205 (1837).

A s cia (part.), Billberg, Enum. Ins., p. 79 (1820).

Mor $p$ ho (part.), Zinken-Sommer, N. Acta Ac. Lsop., v. 15, p. $165,170(1831)$.

Drusilla, Erichson, N. Acta Ac. Leop., v. 16, Suppl., p. 401 (1834).

Thaumantis (part.), E. Doubleday, List Lep. Brit. Mus., pars 1, p. 115 (1844). - E. Doubleday (\& Westwood), Gen. Diurn. Lop., t. 54 (1849); Westwood, 1. c., v. 2, p. 336 (1851). - Chenu \& H. Lucas, Enc. Hist. Nat., Papill,, p. 166 (1851).

II ipparchia ?, Westwood: Donovan, Epitome Ins. Ind., n. ed., p. 45 (1848).

C lerome (Boisduval MS), E. Doubleday (\& Westwood), Gen. Diurn. Lep., t. 54* (1849); Westwood, l. c., v. 2, p. 333 (1851). - Chenu \& H. Lucas, Enc. Hist. Nat., Papill., p. 164 (1851). - Ménétriés, Enum. Corp. Anim. Mus. Petropol., Lep., pars 1, p. 37 (1855). - Horsfield \& F. Moore, Cat. Lep. Mus. E.-Ind. Comp., v. 1, p. 213 (1857). - Westwood, Trans. Ent. Soc. London, n. ser., v. 4, p. 182 (1858). - Hewitson, Exot. Butt., v. 4 (3), t. [1] Clerome (1862). Herrich-Schäffer, Corr.-Bl. Zool.-Min. Ver. Regensb., v. 18, p. 112 ; v. 19 , p. 89 (1864 \& 65). - Clerona (!), id., 1.c., v. 18, p. 132 (1864). - Clerome, A. G. Butler, Ann. Mag. Nat. Hist., ser. 3, v. 20, p. 401 (1867). - Id., Cat. Diurn. Lep. Fabr., p. 44 (1869), - W. F. Kirby, Cat. Diurn. Lep., p. 118 (1871). - C. (part.), Weyenbergh, Pet. Nouv. Ent., 
Année 6, p. 404 (1874). - C., Hopffer, Ent. Zeit. Stettin, v. 35 , p. 38 (1874). - S. H. Scudder, Proc. Amer. Ac. Sci., v. 10, p. 143 (Typ. C. arcesilaus) (1875). - $\mathrm{E}$ Martens, Preuß. Exp. Ost-Asien, v. 1, p. 290 (1875). - F. Müller, Jena. Zeit. Naturw., v. 11, p. 102 (Physiol.) (1877). - Id., Kosmos, Leipzig, v. 1, p. 394 (1877). - F. Moore, Proc. Zool. Soc. London, p. 697, 827 (1878). - G. Semper, Verh. Ver. Naturw. Unterhalt. Hamburg, v. 3, 1876, p. 109 (1878). - P. C. T. Snellen, Tijdschr. Ent., v. 21, p. 11 (1878). - W. F. Kirby, Sci. Proc. R. Dublin Soc., ser. 2, v. 2, p. 300 (1880). - C. Crüger (sec. Kirby), Berlin. Ent. Zeit., v. 25, p. 111 (1881). - Distant, Rhop. Malay., p. 69, 80, 428 (1882 \& 86). - G. F. L. Marshall \& Nicéville, Butt. Ind. Burm. Ceyl., v. 1. II, p. 283, 312 (1883). - B. Gerhard, Berlin. Ent.Zeit., v. 27, p. 181 (distr. geogr.) (1883). - Kheil, Rhop. Nias., p. 20 (1884). - Doherty, Journ. Asiat. Soc. Bengal, v. 55. II, p. 109 (1886). - E. Haasc, Corr.-Bl. Ent. Ver. Iris, v. 1, p. 104, 310 (Organ. odor.) (1886 \& 88). - G. Semper: C. Semper, Reis. Arch. Philipp., pars 2, Schmett., v. 1, p. 68, 331 (1887 \& 92). - L. Glaser, Cat. Etymol. Col. Lep., p. 282 (spec. omiss.) (1887). - O. Staudinger \& Schatz, Exot. Schmott., v. 1, p. 202 (1887). Elwes \& Nicéville, Journ. Asiat. Soc. Bengal, v. 55. II, (1886), p. 419 (1887). - Distant \& Pryer, Ann. Mag. Nat. Hist., ser. 5, v. 19, p. 51 (1887). - Elwes (\& O. Möller). Trans. Ent. Soc. London, p. 282, 334 (1888). - Westwood, l. c., p. 474 (1888). - Röber: O. Staudinger \& Schatz, Exot. Schmett., v. 2, t. 31 (Morphol.), p. 184, 188 (1888 \& 89). - Skertehly, Ann. Mag. Nat. Hist., ser. 6, v. 4, p. 210 (1889). - B. Hagen, Tijdschr. Nederl. Aardr. Gen., ser. 2, v. 7, p. 201 (1890). - A. Seitz, Ent. Zcit. Stettin, v. 51, p. 124 (not. biol.) (1890). - P. O. T. Snellen, Tijdschr. Ent., v. 33, p. 291 (1890). - W. J. Holland, Proc. Boston Soc. Nat. Hist., v. 25, p. 61 (1890). - Nicéville, Journ. Bombay Nat. Hist. Soc., v. 6, p. 344 (1891). - Elwes, Proc. Zool. Soc. London, p. 271 (1891). - P. C. T. Snellen, Not. Leyden Mus., v. 13, p. 157 (1891). - Id., Midden Sumatra, v. 4. VIII, p. 16 (1892). - Leech, Butt. Chin. Jap. Corea, v. 1, p. 112 (1892). - Swinhoe, Trans. Ent. Sac. London, p. 276 (1893). - W. F. Kirby, Handb. Lep. (Allens Natural. Libr.), v. 1, p. 197 (1894). - Nicéville, Gazetteer Sikhim, List Butterfl., p. 131 (1894). - F. Moore, Lep. Ind., v. 2, p. 206, 210 (1895). - Elera, Faun. Filippin., v. 2, p. 269 (1895). - Fürbringer (\& A. Pagonstecher): Sernon, Forschungsreis. 5, Denkschr. Med.-Naturw. Ges. Jena, v. 8, p. (78) 238 (1895). — J. J. Walker, Trans. Ent. Soc. London, p. 450 (1895). - L. Martin, Deutsch. Ent. Zeit. Lep., v. 8, 1895, p. 255 (1896). - E. Reuter, Acta Soc. Sci. Fenn., v. 22, p. 110 (1896). - Nicéville \& L. Martin, Journ. Asiat. Soc. Bencal, v. 64. II, 1895 , p. 396 (1896). - A. Pagenstecher, Abh. Senckenb. Naturf. Ges., v. 23, p. 405 (1897). - Nicéville \& Elwes, Journ. Asiat. Soc. Bengal, v. 66. II, 1897, p. 682 (1898). - Fruhstorfer, Berlin. Ent. Zeit., v. 44, p. 49 (1899). - W. F. Kirby, Entomologist, v, 32, p. 31 (1899). - Hanitsch, Journ. Straits Branch. Asiat. Soc., Nr. 34, p. 83 (1900). - W. F. Kirby: Hübner \& Geyer, Samml. Exot. Schmett., Neue Ausg., v. 3, p. 47 (1901). - Fruhstorfer, Soc. Entom., v. 16, p. 97 (1901). - B. Hagen: A. Maaß, Bei liebenswürd. Wilden, 
Anh., p. 208 (1902). - Nicéville, Journ. Asiat. Soc. Bengal, v. 71. II, p. 8 (1902). - H. Stichel, Deutsch. Ent. Zeit., Iris, v. 15, p. 59 (1902). - Shelford, Journ. Straits Branch Asiat. Soc., Nr. 41, p. 109 (1904). - L. C. H. Young, Journ. Bombay Nat. Hist. Soc., v. 15, 1903, p. 485 (1904). Bingham, Faum. Brit. Ind, Lep., v. 1, p. 181, 182 (Typ. C. arcesilaus) (1905). - Alfken, Abl. Naturw. Ver. Bremen, v. 18, p. 133 (1905). - Fruhstorfer, Soc. Ent., v. 20, p. 34 (1905). - Id., Ent. Zeit., v. 20, p. 98 (1906). A. Pagenstecher, Geogr. Verbr. Schmett., p. 412 (1909, - Bell: Journ. Bombay Nat. Hist. Soc., v. 19, p. 468, 469 (1909). - Tytler, 1. c., v. 21, p. 54 (1911). - TW. H. Evans, 1. c., v. 21, p. 571 (1912). - Eecke, Not. Leyden Mus., v. 35, p. 244 (1913). - W. H. Evans, Journ. Bombay Nat. Hist. Soc., v. 22, 1913, p. 762 (1914). - Id., 1. c., v.1 23, p. 538 (1915). - E. V. Ellis, Tijulschr. Ent., v. 25, p. 107 (1917). - Ollenbach, Journ. Bombay Nat. Hist. Soc., v. 27, p. 885 (1921). - Antram, Butterfl. Ind., p. 117 (1924). - N. D. Riley \& Gabriel, Cat. Type Spec. Rhop. Brit. Mus., pars 1, p. 5 ete. (1924). - Le Cerf, Enc. Ent., ser. B. 3, Lep., v. 1, p. 14 (Morphol.) (1926). - M. E. Fountaine, Entomologist, v. 59 , p. 55 (1926).

Dis cophora (part.), Ménétriés, Dnum. Corp. Anim. Mus. Petropol., pars 1, p. 37 (1855).

M e l a nocyma, Westwood, Tians. Ent. Soc. London, ser. 2, v. 4, p. 186 (1858). - S. H. Scudder, Trans. $\Lambda$ mer. Ac. Sci., v. 10 , p. 215 (Typ. M. faunula) (1875). - G. F. L. Marshall \& Nicéville, Bntt. Ind. Burm. Ceyl., v. 1. II, p. 315 (1883). - Elwes, Proc. Zool. Soc. London, p. 272 (1891). - F. Moore, Lep. Ind., v. 2, p. 210 (1895). - Nicéville, Journ. Bombay Nat. Hist. Sac., v. 8, p. 259 (1895). - E. Y. Watson, 1. c., v. 10, p. 652 (1897). - Bingham, Faun. Brit. Ind., Iep., v. 1, p. 182, 184 (Typ. M. faunula) (1905). - A. Pagenstecher, Geogr. Verbr. Schmott., p. 412 (1909). - T. H. Evans, Journ. Bombay Nat. Hist. Soc., v. 21, p. 571 (1912). - M. (Subgen.), id., 1. c., v. 22, p. 762 (1914).

\section{Sectio Agroeci}

H. Stichel, Gen. Ins., fasc. 36, p. 33 (1906). - Id., Tierreich, Lief. 34 , p. 102 (1912):

\section{Cohors a. Phaoniformes}

H. Stichel, Gen. Ins., fasc. 36, p. 33 (1906). - Id., Tierreich, Lief. 34, p. 102 (1912).

phaon (Erichs.) Drusilla p., Erichson, Nov. Acta Philippinen, Ac. Leop., จ. 16, Suppl., p. 277 (1834). - Sumatra Faunis p., H. Stichel, Gen. Ins., fasc. $36, \mathrm{p}$. 33 (1906). - Fruhstorfer: A. Seitz, Großschmett. Erde, v. 9, p. 405 (1911). - H. Stichel, Tierreich, Lief. 34, p. 101, 102 (1912).

subsp. pha on (Erichs.) Drusilla p., Erichson, Babuyan, ut antea, p. 401 (277), t. 40 (50), f. 1, 2a Luzon, (1a) (O) (1834). - Thaumantis p., (E. Doub- Polillo, leday \&) Westwood, Gen. Diurn. Lep., v. 2, Los Baños ; p. 337 (1851). - Chenu \& H. Lucas, Enc. Hist. Nat. Papill., p. 166 (1851). - Clerome 
p., Westwood, Trans. Ent. Soc. London, ser. 2, v. 4, p. 185 (part.) (1858). - HerrichSchäffer, Corr.-Bl. Zool.-Min. Ver, Regensb., v. 19 , p. $89(1865)$. - W. F. Kirby, Entomologist, v. 10 , p. 292 (1877). - G. Semper: C. Semper, Reis. Arch. Philippin., pars 2. V, Schmett., v. 1, p. 68, 331 (1887 \& 92). O. Staudinger (\& Schatz), Exot. Schmett., v. 1, p. 202 (1887). - F. Moore, Lep. Ind., v. 2, p. 209 (1895). - Elera, Cat. Faun. Filipin., v. 2, p. 269 (1895). - Fruhstorfer, Berlin. Ent. Zeit., v. 44, p. 49 (1899). Shelford, Journ. Straits Branch Asiat. Soc., Nr. 41, p. 109 (part.) (1904). - Fruhstorfer, Sor. Entom., v. 20, p. 14 (1905). Faunis p. p., H. Stichel, Gen. Ins., fasc. 36, p. 33 (1906). - Frulistorfer: A. Seitz, Großschmett. Erde, v. 9, p. 405 (1911). $F$. p. p. forma principalis, $H$. Stichel, Tierreich, Lief. 3t, p. 103, 104 (1912). - F. p., Moulton, Journ. Straits Branch Asiat. Soc., Nr. 65, p. 16 (distrib. part.) (1913). - M. E. Fountaine, Entomologist, v. 59 , p. 55 (1926).

forma ik o nio $n$ Fruhst. F. p. i., Fruhstor- Typ.: ? fer: A. Seitz, Großschmett. Erde, v. 9, p. 405 t. $100 \mathrm{a}(1911)$. - F. p. p. forma $i$., H. Stichel, Tierreich, Lief. 34, p. $104^{\circ}$ (1912).

forma microps (Stauding.) Clerome phaon Typ.: var. m., O. Staudinger (\& Schatz), Exot. Manila Schmett., v. 1, p. 203 (1887). - G. Semper: C. Semper, Reis. Archip. Philippin., pars 2. V, Schmett., v. 1, p. 331 (1892). - C. p. m., Fruhstorfer, Berlin. Ent. Zeit., v. 44 , p. 49 (1899). - C. p. forma temp. m., Fruhstorfer, Soc. Entom., v. 20, p. 34 (1905). - F. p. p. forma m., H. Stichel, Gen. Ins., fase. 36 , p. 33 (1906). - F. p. m., Fruhstorfer A. Seitz, Großschmett. Erde, v. 9 , p. 405 (1911). - F. p. p. forma $m_{\text {., }}$ H. Stichel, 'Tierreich, Lief. 34, p. 104 (1912). subsp. $l u r i d u \&$ (C. \& R. Feld.) Clerome l., Mindoro C. \& R. Felder, Reise Novara, v. 2. II, p. 460 (1866). - W. F. Kirby, Cat. Diurn. Lep., p. 119 (1871). - O. Staudinger (\& Schatz), Exot. Schmett., v. 1, p. 203 (1887). - G. Semper: C. Semper, Reis. Arch. Philippin., pars 2. V, Schmett., v. 1, p. 69, t. 8, f. $19\left(\sigma^{7}\right)$, f. 20 (O) (1887). - F. Moore, Lep. Ind., v. 2, p. 209 (1895). - Elera, Cat. Faun. Filipin., v. 2, p. 269 (1895). C. phaon l., Fruhstorfer, Berlin. Ent. Zeit., v. 44 , p. 49 (1899). - Id., Soc. Entom., v. 20, p. 34 (1905). - F. p. luridus, H. Stichel, Gen. Ins., fasc. 36, p. 33 (1906). - F. p. lurida, Fruhstorfer: 1 . Seitz, Großschmett. Erde, v. 9, p. 405 (1911). - F. p. luridus, H. Stichel, Tierreich, Lief. 34, p. 103, 104 (1912). 
subsp. c arfinia Fruhst. F. p. c., Fruhstor- Guimaras fer: A. Seitz, Großschmett. Erde, v. 9, p. 405 (1911). - H. Stichel, Tierreich, Lief. 34 , p. 103,104 (1912).

subsp. $s u \operatorname{mat} \operatorname{ran} u s \mathrm{~W}$. Rothsch. F. p. s., Sumatra W. Rothschild, Nov. Zool., v. 23, p. 299 (1916).

(O. \& R. Feld.) Clerome l., C. \& R. Mindanao, Felder, Wien. Ent. Monatschr., v. 5, p. 304 Bazilan (1861). - Herrich-Schäffer, Corr.-Bl. Zool.Min. Ver. Regensb., v. 19, p. 89 (1865). C. \& R. Felder, Reise Novara, v. 2. II, p. 460 , t. 62 , ‥ 5,6 (1866). - W. F. Kirby, Cat. Dium. Lep., p. 118 (1871). - G. Semper: C. Semper, Reis. Arch. Philippin., pars 2. V, Schmett., v. 1, p. 69 (1887). - O. Staudinger (\& Schatz), Exot. Schmett., v. 1, p. 203 (1887). - F. Moore, Tep. Ind., v. 2, p. 209 (1895). - Elera, Cat. Faun. Filippin., v. 2, p. 269 (1895). - C. phaon $l$., Fruhstorfer, Berlin. Ent. Zeit., v. 44 , p. 49 (1899). - C. l., id., Berlin. Ent. Zeit., v. 45, p. 13 (1900). - Id., Soc. Entom., v. 20, p. 35 (1905). - Faunis l., H. Stichel, Gen. Ins., fasc. 36, p. 33 (1906). - Fruhstorfer: A. Seitz, Großschmett. Erde, v. 9, p. 405 , t. $100 \mathrm{a}$ (1911). - H. Stichel, Tierreich, Iief. 34, p. 101, 104 (1912).

le ucida (O. Semp.) Clerome l., O. Semper, Verh. Ver. Naturw. Unterhalt. Hamburg, v. v. 3, 1876, p. 108 (1878).

- gracilis (A. G. Buti.) C. g., A. G. Butler, Ann. Malayische Mag. Nat. Hist., ser. 3, v. 20, p. 401, t. 8, Halbinsel, f. 7 (1867). - W. F. Kirby, Cat. Diurn. Sumatra, Lep., p. 119 (1871). - Herb. Druce, Proc. Borneo Zool. Soc. London, p. 341 (1873). - Distant, Rhop. Malay., p. 81, t. 8, f. 1 (1882). - G. F. I. Marshall \& Nicéville, Butt. Ind. Burm. Ceyl., v. 1. II, p. 314 (1853). - O. Staudinger (\& Schatz), Exot. Schmett., v. 1, p. 203 (1887). - Distant \& Pryer, Ann. Mag. Nat. Hist., ser. 5, v. 19 , p. 51 (not. biol.) (1887). - Elwes (\& O. Möller), Trans. Ent. Soc. London, p. 282 (1888). - B. Hagell, Tijdschr. Nederl. Aardr. Gen., ser. 2, v. 7, p. 199 (1890). - Elwes, Proc. Zool. Soc. London, p. 271 (1891). - F. Moore, Lep. Ind., v. 2, p. 209 (1895). - L. Martin, Deutsch. Ent. Zeit. Lep., v. 8, 1895, p. 255 (1896). - Nićsille \& L. Martin, Journ. Asiat. Soc. Bongal, v. 64. II, 1895, p. 397 (1896). - C. phaon g., Frulistorfer, Berlin. Ent. Zeit., v. 44, p. 49 (1899). - C. g., Shelford, Journ. Straits Branch Asiat. Soc., N1. 41, p. 110 (1904). - Frubstorfer, Soc. Entom., v. 20, p. 35 (1905). - Faunis g., H. Stichel, Gen. Ins, fasc. 36, p. 33 (1906). - Fruhstorfer: A. Seitz, GroBschmett. Erde, v. 9, p. 404 , t. $100 \mathrm{~b}$ (1911). - H. Stichel, 
Tierreich, Lief. 34, p. 101, 105 (1912). Moulton, Journ. Straits Branch Asiat. Soc., Nr. 65, p. 17 (1913). - W. Rothschild, Journ. Feder. Malay Stat. Mus., v. 8, p. 153 (1920).

Cle rome phaon (non Drusilla p. Erichson), Horsfield \& F. Moore, Cat. Lep. Mus. E.-Ind. Comp., v. 1, p. 214 (1857). - IVestwood, Trans. Ent. Soc. London, n. ser., v. 4, p. 185 (? part.) (1858). - Herb. Druce, Proc. Zool. Soc. Iondon, p. 341 (1873). - Shelford, Journ. Straits Branch Asiat. Soc., Nr. 41, p. 109 (in cit.) (1904).

menado (Hew.) Clerome m., Hewitson, Exot. Butt., v. 4 (3), t. [1] Clerome (1863). - Faunis m., H. Stichel, Gen. Ins., fasc. 36, p. 33 (1906). - Frulıstorfer: A. Seitz, Großschmett., v. 9, p. 405 (1911). - II. Stichel, Tierreich, Lief. 34, p. 102, 105 (1912).

- subsp. menado (Hew.) Clerome m., ut an- Nord-, tea, fig. 4, 5 (1863). - Herrich-Schäffer, MittelCorr.-Bi. Zool.-Min. Ver. Regensb., v. 19, Celebes p. 89 (1865). - IV. F. Kirby, Cat. Diurn. Lep., p. 119 (1871). - Hopffer, Ent. Zeit. Stettin, v. 35 , p. 38 (1874). - O. Stuudinger (\& Schatz), Exot. Schmctt., v. 1, p. 202 (? part.) (1887). - Fruhstorfer, Berlin. Ent. Zeit., v. 44, p. 50 (1899). - C. m. m., id., Soc. Entom., v. 20, p. 35 (1905). - Faunis m. m., H. Stichel, Gen. Ins., fasc. 36 , p. 33 , t. 5 , f. 11 () (1906). - Fruhstorfer: A. Seitz, Grofschmett. Erdc, v. 9, p. 405 (1911). - F.m. m. forma principalis, H. Stichel, Ticrreich, Lief. 34, p. 107 (1912). - Clerome m., N. D. Riley \& Gabriel, Cat. Type Spec. Rhop. Brit. Mus., pars 1, p. 36 (1924).

? Clerome chitone, Hopffer, Ent. Zeit. Stettin, v. 35, p. 38 (1874).

forma $z$ en i ca Frulist. F. m. z., Fruhstorfer: Typ.:

A. Seitz, Großschmett. Ende, v. 9, p. 405 Mittel-

(Trockenzeitform) (1911). - F.m. m. forma Celebes z., H. Stichel, Tierreich, Lief. 34, p. 108 (1912).

subsp. intermedius (Röb.) Clerome in. Bangkai termedia, Röber, Entom. Nachr., v. 22, p. (nördl. 172 (1896). - C. menado i., Fruhstorfer, Celebes)

Berlin. Ent. Zeit., v. 44, p. 50 (1899). Faunis m. $i_{\text {. }}$ II. Stichel, Gen. Ins., fasc. 36, p. 34 (1906). - Fruhstorfer: A. : Seitz, Großschmett. Erde, v. 9, p. 405 (1911). H. Stichel, Tierreich, Lief. 34, p. 107, 108 (patria corrigend.) (1912).

subsp. pleo n asma (Röb.) Clerome p., Rö- Ost-Celebes ber, Entom. Nachr., v. 22, p. 171 (1896). C. menado p., Fruhstorfer, Berlin. Ent. Zeit., v. 44, p. 50 (1899). - Faunis m. p., II. Stichel, Gen. Ins., fasc. 36, p. 34 (1906). - 
Fruhstorfer: A. Seitz, Großschmett. Erde, v. 9, p. 405 (1911). - H. Stichel, Tierreich, Lief. 34, p. 107, 108, fig. 17 (1912).

subsp. ch it o ne (Hew.) Clerome c., Hewit- Süd-Celebes son, Exot. Butt., v. 3 (4), t. [1] Clerome, f. 2, 3 (1862). - Herrich-Schäffer, Corr.Bl. Zool.-Min. Ver. Regensb., v. 19, p. 89 (1865). - W. F. Kirby, Cat. Diurn. Lep., p.119 (1871). - C. c. (C. celebensis Snellen v. Vollenhofen MS), P. C. T. Snellen, Tijdschr. Entom., v. 21, p. 11 (1878). - E. Haase, Corr.-Bl. Ent. Ver. Iris, v, 1, p. 104, 310 (Organ. odor.) (1886 \& 88). - O. Staudinger (\& Schatz), Exot. Schmett., v. 1, t. 65 (O); p. 202 (1886 \& 87). - Holland, Proc. Boston Soc. Nat. Hist., v. 25, p. 61 (1890). - W. Rothschild, Deutsch. Ent. Zeit. Lep., v. 5, p. 434 (1892). - C. menado c., Fruhstorfer, Berlin. Ent. Zeit., v. 44, p. 50 (1899). - Faunis m. c., H. Stichel, Gen. Ins., fasc. 36, p. 34 (1906). - Frulsstorfer: A. Seitz, Großschmett. Erde, v. 9, p. 405 (1911). $F$. m. c. forma principalis, $H$. Stichel, Tierreich, Lief. 34, p. 107, 109, 110 (1912). Clcrome c., N. D. Riley \& Gabriel, Cat. Typo Spec. Rhop. Brit. Mus., pars 1, p. 14 (1924).

Clerome menado, A. Pagenstecher. Abh. Senckenb. Naturf. Ges., v. 23, p. 406 (1897). forma Fruhstorferi (Pöb.) Clerome f., Röber, Entom. Nachr., v. 22, p. 171 (1896). - C. menado f., Fruhstorfer, Berlin. Ent. Zeit., v. 44, p. 50 (1899). - Faunis $m$. chitone forma $f$., H. Stichel, Gen. Ins., fasc. 36, p. 34 (1906). - F. m. f., Fruhstorfer: A. Seitz, Großschmett. Erde, v. 9, p. 405 (Bergform), t. 100 a (1911). $-F$. m. chitone

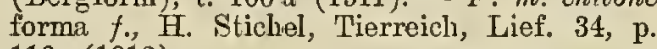
110 (1912).

subsp. syllus Fruhst. F. m. s., Fruhstorfer: Sangir A. Seitz, Großschmett. Erde, v. 9, p. 405 (1911). - H. Stichel, Tierreich, Lief. 34, p. 107, 108 (1912).

subsp. sulanus Fruhst. Clerome menado sulana, Fruhstorfer, Berlin. Ent. Zeit., v. 44, p. 49, 50 (1899). - C. s., Nicéville, Journ. Bombay Nat. Hist. Soc., v. 13 , p. 165, t. DD, f. 9 (ㅇ) (1900). - Faunis m. sulanus, H. Stichel, Gen. Ins., fasc. 36 , p. 31 (1906). $F$. m. sulwana (!), Fruhstorfer: A. Seitz, Großschmett. Erde, v. 9, p. 405 (1911). $F$. m. sulanus, II. Stichel, Tierreicb, Lief. 34 , p. 107,110 (1912).

sappho (G. Semp.) Clerome s., G. Semper, Verh. Philippinen Ver. Naturw. Unterh. Hamburg, v. 3, 1876, p. 108 (1878). - Faunis s., H. Stichel, Gen. Ins., fasc. 36, p. 34 (1906). - Fruhstorfer: A. Seitz, Großschmett. Erde, v. 9, p. 406 (1911). - H. Stichel, Tierreich, Lief. 34, p. 102, 110 (1912). 
subsp. sappho (G. Semp.) Clerome s., G. Mindanao, Semper, ut antea (1878). - Id., Reis. Arch. Bohol Philippin., pars 2. V, Schmett., p. 60 (part.) (1887). - O. Staudinger (\& Schatz), Exot. Schmett., v. 1, p. 203 (1887). - F. Moore, Iep. Ind., v. 2, p. 209 (1895). - Elera, Cat. Faun. Filipin., v. 2, p. 269 (1895). Fruhstorfer, Berlin. Ent. Zeit., v. 44, p. 50 (1899). - Id., Sac. Entom., v. 20, p. 35 (1905). - Faunis s. s., II. Stichol, Gen. Ins., fasc. 36, p. 34 (1906). - Frulistorfer: A. Seitz, Großschmett. Erde, v. 9, p. 406 (1911). - H. Stichel, Tierreich, Iief. 34, p. 111 (1912).

subsp. a mé $i$ a al $l$ e $i$ a Fruhst. F.s.a., Fruh- Camiguin storfer: A. Seitz, Großschmett. Erde, v. 9, p. 406 (1911). - II. Stichel, Tierreich, Iief. 34, p. 111 (1912).

subsp. kleis (G. Semp.) Clerome k., G. Camotes, Semper, Verh. Ver: Naturw. Unterh. Ham- Samar, burg, v. 3, 1876, p. 109 (1878). - Id.: O. Panaon, Semper, Reis. Areh. Philippin., pars 2. V, Siargao Schmett., v. 1, p. 70, t. 8 , f. $17,18\left(\sigma^{7}\right.$, 우) (1887). - O. Staudinger (\& Schatz), Exot. Schmett., v. 1, p. 202 (1887). - F. Moore, Lep. Ind., v. 2, p. 209 (1887). - Elera, Cat. Faun. Filipin., v. 2, p. 269 (1895). Fruhstorfer, Berlin. Ent. Zeit., v. 44, p. 50 (1899). - C. phaon (?) k., id., Soc. Entom., v. 20, p. 35 (1905). - Faunis s. k., H. Stichel, Gen. Ins., fasc. 36, p. 31 (1906). Frulsstorfer: A. Seitz, Großschmett. Erde, v. 9, p. 406 (1911). - H. Stichel, Tierreich, Iief. 34, p. 111 (1912).

stomphax (Westw.) Clerome s., Westwood, Trans. Borneo, Ent. Soc. London, n. ser., v. 4, p. 186 (1858). - Faunis s., H. Stichel, Gen. Ins., fasc. 36, p. 34 (1906). - Fruhstorfor: A. Seitz, Großschmett. Erde, v. 9, p. 404 (1911). II. Stichel, Tierreich, Tief. 34 , p. 102,112 (1912).

subsp. s tomphax (Westw.) Clerame s., Borneo, Westwood, ut antea, t. 21, f. 3, 4 (1858). - Billiton, Herrich-Schäffer, Corr.-Bl. Zool.-Min. Ver. Sumatra Riogensb., v. 19 , p. 89 (1865). - W. F. Kirby, Cat. Diurn. Lep., p. 119 (1871). Herb. Druce, Proc. Zool. Soc. London, p. 341 (1873). - C. stomphae (!), W. F. Kirby, Entomologist, v. 10, p. 392 (1877). - C. stomphax, $O$. Staudinger (\& Schatz), Exot. Schmett., v. 1, p. 203 (1887). - P. O. T. Snellen, Tijdschr. Entom., v. 33, 1889, p. 291 (1890). - Id., Not. Leyden Mus., v. 13, p. 137 (1891). - F. Moore, Lep. Ind., v. 2, p. 209 (1895). - A. Pagenstecher, Abh. Senckenb. Naturf. Ges., v. 23, p. 406 (1897). - Fruhstorfer, Berlin. Ent. Zeit., v. 44, p. 50 (1899). - Shelford, Journ. Straits Branch Asiat. Soc., Nr. 41, p. 110 (1904). -

Sumatra,

Palawan, Pulo Laut

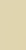


C. s., C. s. nov. subsp., Fruhstorfer, Soc. Entom., v. 20, p. 35 (1905). - Faunis s. s., H. Stichel, Gen. Ins., fasc. 36, p. 34 (1906). - Fruhstorfer: A. Seitz, Großschmett. Erde, v. 9 , p. 404, t. 100 b (1911). - F. s. s. forma principalis, H. Stichel, Tierreicl, Lief. 34, p. 113 (1912). - F. 8., Moulton, Joum. Straits Branch Asiat. Soc., Nr. 65, p. 17 (distr. part.) (1913). - Clerome s., N. D. Riley \& Gabriel, Cat. Type Spcc. Rhop. Brit. Mus., pars 1, p. 55 (1924).

- forma besa (Hew.) Clerome b., Hewitson, Typ.:

Exot. Butt., v. 4 (3), t. [1] Clerome, f. Borneo 1 (1862). - Horrich-Schäffer, Corr.-Bl. Zool. Min. Ver. Regensb., v. 19, p. 89 (1865). W. F. Kirby, Sci. Proc. R. Dublin Soc., ser. 2, v. 2, p. 300 (1880). - Id., Cat. Diurn. Iep., p. 119 (1871). - O. Staudinger (\& Schatz), Exot. Schmett., v. 1, p. 203 (1887).

- F. Moore, Lep. Ind., v. 2, p. 209 (1895).

- C. stomphax ab. b., Fruhstorfor, Berlin. Ent. Zeit., v. 14, p. 50 (1899). - C. b., Hanitsch, Journ. Straits Branch Asiat. Soc., Nr. 34, p. 83 (1900). - Shelford, 1. c., Nr. 41, p. 110 (1904). - C. stomphax forma beza (!), Fruhstorfer, Soc. Entom., v. 20, p. 35 (? Talform) (1905). - F. s. s. forma besa, H. Stichel, Gen. Ins., fasc. 36 , p. 34 (1906). - F. 8. b., Fruhstorfer: A. Seitz, Großschmett. Erde, v. 9, p. 404 (1911). $F$. \&. 8. forma $b$, H. Stichel, Tierreich, Lief. 34 , p. 113 (1912). - $F$. s. ,form ${ }^{6}$ b. Moulton, Journ. Straits Branch. Asiat. Soc., Nr. 41, p. 17 (1913). - Clerome b., N. D. Riley \& Gabriel, Cat. Type Spec. Rhop. Brit. Mus., pars 1, p. 10 (1924).

forma $B$ ar ra ut $i$ Moult. $F$. s. b., Moulton, 1yp.:

Entomologist, v. 48, p. 99 (1915). Nord-

Borneo

(Kina Balu)

subsp. la $u t$ en sis W. Rothsch. F. s. l., W. Pulo Laut Rothschild, Nov. Zool., v. 23, p. 299 (1916). subsp. Plateni (O. Stauding.) Clerome p., Palawan

O. Staudinger, Deutsch. Ent. Zoit. Lep., v. 2, p. 44 (1889). - G. Semper: O. Semper, Rois. Arch. Philippin., pars 2. V, Schmett., v. 1, p. 331 (1892). - F. Moore, Lep. Ind., v. 2, p. 109 (1895). - Elera, Cat. Fann. Filipin., v. 2, p. 269 (1895). - C. stomphax p., Fruhstorfer, Berlin. Ent. Zeit., v. 44, p. 50 (1899). - C. p., Fruhstorfer, Soc. Entom., v. 20 , p. 35 (1905). - Faunis stomphax $p$., H. Stichel, Gen. Ins., fasc. 36, p. 35 (1906). - Fruhstorfer: A. Soitz, Großschmett. Erde, v. 9, p. 405 (1911). - H. Stichel, Tierreich, Lief. 34, p. 112, 113 (1912). - Moulton, Journ. Straits Branch Asiat. Soc., Nr. 65, p. 17 (1913). 


\section{Cohors b. Eumeiformes}

Arcesila iformes, H. Stichel, Gen. Ins., fasc. 36,p. 35 (1906). Id., Tierreich, Lief. 34, p. 113 (1912).

canens Jac. Hübn. $F$. c, Jac. Hübner, Samml. Exot. Schmett., v. 2, t. [82] [1820-26].

Java u. a.

F. arcesilaus (non Pap. a. Fabricius), H. Inseln,

Stichel, Gen. Ins., fasc. 36, p. 35 (1906). - Hinter-

Fruhstorfer: $\Lambda$. Seitz, Großschmett. Erde, Indien, v. 9 , p. $40 \pm$ (1911). - H. Stichel, Tier- Nordreich, Lief. 34 , p. 102, 113 (1912). - Eecke, Indien

Not. Leyden Mus., v. 36, p. 228 (1914). -

Id., Zool. Mededeel. Lcirlen, v. 4, p. 83 (1918).

subsp. c anens Jac. Hübner $F$. c., Jac. Java

Hübner, ut antea, t. [82], f. 3,4 [1820-26].

- W. F. Kirby: Hübner \& Geyer, Samml.

Exot. Schmett., Neue Ausg., v. 2, t. 295 (82),

f. $3,4(1898)$. - Clerome arcesilaus caneus (!), Fruhstorfer, Soc. Entom., v. 20, p. 34. 35 (1905). - Fuunis a. canens, H. Stichel, Gen. Ins., fasc. 36, p. 115 (1906). Fruhstorfer: A. Seitz, Großschmett. Erde, v. 9, p. 404 (1911). - F. a. c. forma principalis, H. Stichel, Tierreich, Lief. 34, p. 114, 115 (1912). - F. a. c., Eocko, Not. Leyden Mus., v. 36, p. 228 (1916).

le onteus (Zink.-Somm.) Morpho l., ZinkenSommer, Nov. Acta Ac. Treop., v. 15, p. 170 t. $16, \mathrm{f} .14,15$ (1831).

Discophora arcesilaus, Ménétriés, Enum. Corp. Anim. Mus. Petropol., pars 1, p. 37 (1855). - Clerome a., Horsfield \& F. Moore, Cat. Lep. Mus. E.-Ind. Comp., v. 1, p. 213 (cit. part.) (1857), - A. G. Butler, Cat. Diurn. Lop. Fabr., p. 44 (in cit.) (1869). - Fruhstorfer, Berlin, Ent. Zeit., v. 41, p. 301 (1897). - W. F. Kirby: Hübner \& Geyer, Samml. Exot. Schmett., Neue Ausg., v. 3, p. 47 (cit. part.) (1901). - Alfken, Abh. Naturw. Ver. Bremen, v. 18, p. 133 (1905).

forma c y me Fruhst. F. arcesilaus c., Fruh- Typ.: storfer: A. Seitz, Großschmett. Erde, v. 9, p. 449 (1911). - F. a. canens forma c., H. Stichel, Tierreich, Lief. 34, p. 115 (1912), F. a. c., Eecke, Not. Leyden Mus., v. 36, p. 228 (1916).

subsp. borneensis (Fruhst.) Clerome arce- Borneo, silaus b., Fruhstorfer, Soc. Entom., v. 20, p. Natuna 35 (1905). - Faunis a. b., H. Stichel, Gen. Ins., fasc. 36, p. 35 (1906). - Fruhstorfer: 1. Seitz, Großschmett. Erde, v. 9, p. 404 (1911). - H. Stichel, Tierroich, Licf. 34, p. 114, 115 (1912). - Moulton, Journ. Straits Branch Asiat. Soc., Nr. 36, p. 228 (1913). Eecke, Not. Leyden Mus., v. 36, p. 228 (1914).

Clerame arcesilaus, Herb. Druce, Proc. Zool. Soc. London, p. 341 (1873). - A. 
Pagenstecher, Abh. Senckenb. Naturf. Ges., v. 23, p. 405 (1897). - Shelford, Journ. Straits Branch Asiat. Soc., Nr. 41, p. 110 (1904). subsp. baliensis W. Rothsch. F. arcesilaus Bali b., W. Rotbschild, Nov. Zool., v. 23, p. 299 (1916).

Clerome arcesilaus, Nicóville \& Elwes, Journ. Asiat. Soc. Bengal, v. 66. II, 1897, p. 682 (1898). - W. Rotbscbild, Nov. Zool., v. 22, p. 124 (1915).

subsp. bankensis W. Rothsch. F. arcesi- Bangka

laus b., W. Rotliscbild, Nov. Zool., v. 23, p. 299 (1916).

Clerome arcesila"s, Weyenbergh, Pet.

Nouv. Fntom., Annéo 6, p. 404 (1874).

subsp. niasanus (Fruhst.) Clerome arcesi- Nias

laus niasana, Fruhstorfer, Berlin. Ent. Zeit., v. 44, p. 50,51 (1899). $\rightarrow$ Id., Soc. Entom., v. 20, p. 35 (1905). - Faunis a. niasanus, H. Sticbel, Gen. Ins., fasc. 36, p. 35 (1906). - F. a. niasana, Fruhstorfer: A. Seitz, Großscbmett. Erde, v. 9, p. 404 , t. 100 b (1911). - F. a. niasanus, H. Stichel, Tierreicl. Lief. 34, p. 114,116 (1912). - F. $a$. niasana, Eecke, Not. Teyden MLus., v. 36 , p. 228 (1914).

Clerome arcesilaus, Kheil, Rhop. Nias, p. 20 (1884).

subsp. pallidior (B. Hag.) Clerome arce- Mentawei silaus var. p., B. Hagen, Abb. Senckenb. Na- Inseln

turf. Ges., v. 20, p. 331 (1902). - Id., Bei liebenswürd. Wilden, Arihang, p. 208 (1902). - Faunis a. p., H. Stichel, Gen. Ins., fasc. 36, p. 35 (1906). - Frubstorfer: A. Seitz, Großscbmett. Erde, v. 9, p. 404 (1911). H. Stichel, Tierreich, Lief. 34, p. 114, 116 (1912). - Eecke, Not. Leyden Mus., v. 36, p. 228 (1914).

subsp. tenuitata Eecke $F$. arcesilaus t., Pulu Babi, Fecke, Not. Teyl. Mus., v. 36, p. 228 (1914). Pulu Lasia - Id., Zool, Mededeel. Leiden, v. 4, p. 83 (Simalur) (1918).

Clerome arcesilaus var. besa (non $C$. b. Hewitson), Eecke, Not. Ioyden Mus., v. 35 , p. 241 (1913).

subsp. s a $m a d h i$ Frulst. F. arcesilaus s., Batu

Fruhstorfer, Ent. Zeit. Guben, v. 20, p. 146 (1906). - Id.: A. Seitz, Großschmott. Erde, v. 9 , p. 404 (1911). - H. Sticbel, Tierreich, Lief. 34 , p. 114, 116 (1912). - Eecke, Not. Teyden Mus., v. 36 , p. 228 (1914).

- subsp. arcesilas, nov. nomen. Papilio ar- Sikkim, cesilaus (praeocc.: Pap. a. Sulzer, 1776; Pap. Assam, a., Stoll, 1782), J. O. Fabricius, Mant. Ins., v. 2, p. 28 (1787). - P. (Danaus) a., Gmelin-Linné, Syst. Nat., ed. 13, v. 1. V, p. 2281 (1790). - P. a., Jung, Alphab. Verz. Scbmett., v. 1, p. 46 (1791). - J. O. Fabricius, Ent. Syst., v. 3. I, p .153 (1793). -

Inseln

Burma-

Malayiscbe

Halbinsel,

Siam,

Tonkin,

Sumatra 
Latham \& H. Davis: J. A. Forster, Zool. Index, ed. 2, pars 3, p. 25 (1795). - Donovan, Epitome Ins. Ind., t. 30, f. 2 (1800). Turton, Gen. Syst. Nat. Linné, v. 3. II, p. 117 (1806). - Satyrus a., (Latreille \&) J. B. Godart, Enc. Meth., v. 9, p. 497 (1824). Thaumantis a., E. Doubleday, List Lep. Brit. Mus., pars 1, p. 115 (1844). - Hipparchia? a., Westwood: Donovan, Epitome Ins. Ind., n. ed., t. 30 , f. 2 ; p. 45 (1848). - Clerome a., F. Doubleday (\& Westwood), Gen. Diurn. Lep., t. 54*, f. 5 (1849); Westwood, v. 2, p. 334 (distr. part.) (1851). - Chenu \& H. Lucas, Enc. Hist. Nat., Papill., p. 164, f. 276, p. 165 (1851). - Discophora a., Ménétriés, Enum. Corp. Anim. Mus. Petropol., pars 1 , p. 37 (1855). - Westwood, Trans. Ent. Soc. London, n. ser., v. 4, p. 183 (part.) (1858). - Herrich-Schäffer, Corr.Bl. Zool.-Min. Ver. Regensb., v. 19, p. 89 (1865). - A. G. Butler, Cat. Diurn, Lep. Fabr., p. 44 (in cit.) (1869). - W. F. Kirby, Cat. Diurn. Lep., p. 118 (1871). - Id., Entomologist, v. 10 , p. 292 (1877). - F. Moore, Proc. Ent. Soc. Iondon, p. 827 (1878). - W. F. Kirby, Sci. Proc. R. Dublin Soc., ser. 2, v. 2, p. $300(1880)$ - G. F. L. Marshall \& Nicéville, Butt. Ind. Burm. Ceyl., v. 1. II, p. 313 (cit. part.) (1883). - E. Haase, Corr.-Bl. Ent. Ver. Iris, v. 1, p. 104 (Organ. odor.) (1886). - Distant, Rhop. Malay., p. 428 (distr. part.), t. 40, f. 5 (1886). - Elwes \& Nicéville, Journ. Asiat. Soc. Bengal, v. 55. II, 1886, p. 419 (1887). O. Staudinger (\& Schatz), Exot. Schmett., v. 1 , t. 65 , p. 202 (part.) (1886 \& 87). - Elwes (\& O. Möller), Trans. Ent. Soc. London, p. 334 (1888). - B. Hagen, Tijdschr. Nederl. Aardr. Gen., ser. 2, v. 7, p. 199, 201 (1890). - Elwes, Proc. Zool. Soc. London, p. 271 (1891). - B. Hagen, Berlin. Ent. Zeit., v. 37, p. 144 (1892). - P. C. T. Snellen, Midden Sumatra, v. 4. VIII, p. 16 (1892). Swinhoe, Trans. Ent. Soc. London, p. 276 (1893). - Nicéville, Gazetteer Sikhim, List Butterfl, p. 131 (1894). - F. Moore, Lep. Ind., v. 2, p. 207, t. 162 , f. $1,1 \mathrm{a}-\mathrm{o}\left(\sigma^{\prime}, \phi^{\prime}\right)$ (1895). - L. Martin, Deutsch. Ent. Zeit. Iep., v. 8, 1895, p. 255 (1896). - Nicéville \& L. Martin, Journ. Asiat. Soc. Bengal, v. 64. II, 1895, p. 396 (1896). - Iruhstorfer, Berlin. Ent. Zeit., v, 44, p. 51 (part.) (1899). - W. F. Kirby: Hübner \& Geyer, Samml. Exot. Schmett., Neue Ausg., v. 3, p. 47 (in cit.) (1901). - L. C. H. Joung, Journ. Bombay Nat. Hist. Soc., v. 15, 1903, p. 485 (in cit. falso $C$. camens, $C$. leontius) (1904).Bingham, Faun. Brit. Ind., Butterfl., v. 1, p. 183 (1905). - C. a. a., Fruhstorfer, Soc. 
Entom., v. 20, p. 35 (1905). - Faunis a. a., H. Stichel, Gen. Ins., fasc. 36, p. 35 (1906). - Fruhstorfer: A. Seitz, Großschmett. Erde, v. 9, p. 404 (1911). - Clerome a., Tytler, Journ. Bombay Nat. Hist. Soc., v. 21 , p. 54 (1911). - Faunis a. a., H. Stichel, Tierreich, Lief. 34, p. 115 (1912). - Clerome a., W. H. Evans, Journ. Bombay Nat. Hist. Soc., v. 21, p. 571 (1912). - Faunis a. a., Eecke, Not. Leyden Mus., v. 36, p. 228 (1914). - Godfrey, Journ. Nat. Hist. Soc. Siam, v. 2, p. 123 (1916). - Clerome a., Ellis, Journ. Bombay Nat. Hist. Soc., v. 25, p. 107 (1917). - Faunis a. a., Eecke, Zool. Mededeel., v. 4, p. 83 (1918). - W. Rothschild, Journ. Feder. Malay Stat. Mus., v. 8, p. 153 (1920). - Clerome a., Ollenbach, Journ. Bombay Nat. Hist. Soc., v. 27, p. 887 (1921). - Faunis a., W. H. Evans, 1. c., v. 29, p. 85 , t. 15 , fig. E 2. 1. (1923). Papilio a., N. D. Riley \& Gabriol, Cat. Type Spec. Rhop. Brit. Mus., pars 1, p. 8 (1924). - Clerome a., Antram, Butterfl. Ind., p. 117, f. 212 (1924). - Faunis a., W. H. Evans, Identif. Ind. Butterfl., p. 85, t. 15, fig. E 2. 1 (1927). - Tytler, Journ. Bombay Nat. Hist. Soc., v. 31, p. 259 (1927). - F. a. a., Godfrey, Journ. Siam Soc. Nat. Hist., v. 7, p. 122 (1927). - F. a., Rosa, Entomologist, v. 62 , p. $226(1929)$. - F. a. a., Godfrey, Journ. Siam Soc. Nat. Hist. Suppl., v. 7, p. 257 (1930).

kirata (Nicév.) Clerome $k$. (Doherty MS), Nicé- Malakka, ville, Journ. Bomhay Nat. Hist. Soc., v. 6, p. Sumatra, 344, t. F, fig. 3 (1891). - Swinhoe, Trans. Simalur, Ent. Soc. London, p. 276 (1893). - F. Moore, Borneo Lep. Ind., v. 2, p. 209 (1895). - L. Martin, Deutsch. Ent. Zeit. Lop., v. 8, 1895, p. 255 (1896). - Nicéville \& I. Martin, Journ. Asiat. Soc. Bengal, v. 64. II, 1895, p. 397 (1896). - Fruhstorfer, Berlin. Ent. Zoit., v. 44, p. 51 (1899). - Hanitsch, Journ. Straits Branch Asiat. Soc., Nr, 34, p. 83 (1900). - Shelford, I. c., Nr. 41, p. 110 (1904). - Fruhstorfer, Soc. Entom. v. 20 , p. 35 (1905). - Faunis k., H. Stichel, Gen. Ins., fase. 36, p. 35 (1906). - Fruhstorfer: A. Seitz, Großschmett. Erie, v. 9, p. 404 , t. $100 \mathrm{~b}(1911)$ - - H. Stichel, Tierreich, Liof. 34, p. 102,116 (1912). - Moulton, Journ. Straits Branch Asiat. Soc., Nr. 65, p. 17 (1913). - Eecke, Zool. Mededeel. Leiden, v. 4, p. 83 (1918).

eumeus (Drury) Papilio e., Drury, Ill. Nat. Hist., Süd-China, v. 1, p. 4 (1770). - Faunis e., H. Stichel, Hainan, Gen. Ins., fasc. 36, p. 35 (1906). - Fruh- Siam, storfer: A. Seitz, Großschmett. Erde, v. 9, p. Annam, 406 (1911). - H. Stichel, Tierreich, Lief. Shan34, p. 102,117 (1912).

Staten 
subsp. e umeus (Drury) Papilio e., Drury, Süd-China, ut antea, t. 2, f. 3 (1770). - "Eumea", P. Hainan

e., P. Cramer, Pap. Exot., v. 2, p. 132, t. 183; f. C, D; p. 148 (1777). - P. e., O. Stoll, Essai Syst. Iep.: P. Cramer, Pap. Exot., v. 4, Append., p. 9 (1782). - Jung, Alphab. Verz. Schmett., v. 1, p. 202 (1791). - Faunis eumea, Jas. Hübner, Verz. Schmett., p. 55 [1818]. - Satyrus e., Verloren, Cat. Ins., Lep. Cramer, v. 1, p. 75 (1837). - Thaumantis e., E. Doubleday, Iist Top. Brit. Mus., pars 1, p. 115 (1844). - Clerome eumeus, (E. Doubleday \&) Westwood, Gen. Diurn. Lep., v. 2, p. 334 (distr. part.) (1851). - Chenu \& II. Lucas, Enc. Hist. Nat., Papill., p. 165 (1851). - Ménétriés, Enum. Corp. Ánim. Mus. Petropol., pars 1, p. 37 (1855). - Westwood, Trans. Ent. Soc. London, n. ser., v. 4, p. 183 (1858). - HerrichSchäffer, Corr.-H1. Zool.-Min. Ver. Regensb., v. 19, p. 89 (1865). - ? A. G. Butler, Cat. Diurn. Lep. Fabr., p. 44 (1869). - W. F. Kirby, Cat. Diurn. Lep., p. 119; Suppl.; p. 714 (1871 \& 77). - Id., Entomologist, v. 10, p. 292 (1877) - F. Moore, Proc. Zool. Soc. London, p. 697 (1878). - W. F. Kirby, Sci. Proc. R. Dublin Soc., ser. 2, v. 2, p. 300 (1880). - O. Staudinger (\& Schatz), Exot. Schmett., v. 1, p. 202 (1887). - 1 . Seitz, Ent. Zeit. Stettin, v. 51, p. 124 (1890). J. J. Walker, Trans. Ent. Soc. London, p. 442,450 (larva, not. biol.) (1895). - T. Moore, Iep. Ind., v. 2, p. 209 (1895). Fruhstorfer, Berlin. Ent. Zeit., v. 44, p. 51 (1899). - W. F. Kirby, Entomologist, v. 32, p. 31 (1899). - Nicéville, Journ. Asiat. Soc. Bengal, 71. II, p. 8 (1902). - Kershaw, Trans. Fint. Soc. London, p. 56 (1903). - Bingham, Faun. Brit. Ind. Butterfl., v. 1, p. 183 (in cit.) (1905). - C. e. e., Fruhstorfer, Soc. Entom., v. 20, p. 35 (1905). Faunis e. e., H. Stichel, Gen. Ins., fasc. 36, p. 35 (1906). - Fruhstorfer: A. Seitz, Großschmett. Ende, v. 9, p. 406 (1911).H. Stichel, Tierreich, Iief. 34, p. 118 (1912). - Joicey \& Talbot, Bull. Hill Mus., v. 2, p. 184 (1928).

gripus (Fabr.) P. g., J. C. Fabricius, Syst. Ent., p. 829 (1775). - P. (Danaus) g., Goeze, Ent. Beytr., v. 3. I, p. 209 (1779). $P$. g., J. C. Fabricius, Spec. Ins., v. 2, p. 58 (1781). - P. grispus (!), id., Mant. Ins., v. 2 , p. 28 (1787). - $P$. (Danaus) g., GmelinLinné, Syst. Nat., ed. 13, v. 1. V, p. 2280 (1790). - $P$. g., Jung, Alphab. Verz. Schmett., v. 1, p. 250 (1791). - (Jablonsky \&) Herbst, Naturs. Ins., Schmett., v. 6, p. 77 , t. 138, f. 3,4 (1793). - Turton, Gen. Syst. Nat. Linné, v. 3. II, p. 117 
(1806). - Ascia g., Billberg, Enum. Ins., p. 79 (1820). - Satyrus g., (Latreille \&) J. B. Gorlart, Enc. Méth., v. 9, p. 497 (1824). - Verloren, Cat. Syst. Lep. Cramer, v. 2, p. 205 (1837). - Papilio g., Kerr, Glasgow Natural., ser. 3, จ. 2, p. 108 (typ. coll. Hunter) (1910).

decempunctatus (Goeze) Papilio (Danaus) d., Goeze, Entom. Beytr., v. 3. I, p. 212 (1779).

subsp. incertus (O. Stauding.) Clerome Tonkin, eumeus var. i., O. Staudinger (\& Schatz), Annam, Exot. Schmett., จ. 1, p. 202 (patria incerta) Shan(1887). - C. e. i., Fruhstorfer, Berlin. Staaten, Ent. Zeit., จ. 44, p. 51 (1899). - Id., Soc. Burma Entom., v. 20, p. 35 (1905). - Faunis e. incertus, H. Stichel, Gen. Ins., fasc. $36, \mathrm{p}$. 36 (1906). - F. eumeus incerta, Fruhstorfer: A. Seitz, Großschmett. Lirde, v. 9, p. 406 , t. 100 a (1911). - F. e. incertus forma principalis, H. Stichel, Tierreich, Lief. 34, p. 118, 119 (1912). - Clerome e. "race" incerta, W. H. Evans, Journ. Bombay Nat. Hist. Soc., จ. 21, p. 571 (1912). - Faunis e. i., id., I. c., v. 29 , p. 794 (1923). - Id. Identif. Ind. Butterfl., p. 85 (1927). - Godfrey, Journ. Siam Soc. Nat. Hist., Suppl., v. 7, p. 257 (? part.) (1930).

? Clerome eumeus (non Pap. e. Drury), A. G. Butler, Cat. Diurn. Lep., p. 44 (cit. except.) (1869). - C. e., Bingham, Faun. Brit. Ind., Butterfl., v. 1, p. 183 (cit. except., distr. part.) (1905).

? C. as sama (non Westwood), Ch. Oberthür, Etud. Entom., v. 17, p. 13 (1893).

forma moiar um (Fruhst.) Clerome e. for- Tyр.: ma $m$., Fruhstorfer, Soc. Entom., v. 20, p. 34, Annam 35 (1905). - Faunis e. incertus forma m., H. Stichel, Gen. Ins., fasc. 36, p. 36 (1906). - F. e. m., Fruhstorfer: A. Seitz, Großschmett. Erde, v. 9, p. 406 (1911). - F. e. incertus forma m., H. Stichel, Tierreich, Lief. 34, p. 118, 119 (1912).

? F.e. in certa, Godfrey, Journ. Feder. Malay Stat. Mus., v. 3, p. 467 (1919). - Id., Journ. Siam Nat. Hist. Soc., Suppl., v. 7, p. 257 (part.) (1930).

- assamus (Westw.) Clerome assama, Westwood, Trans. Ent. Soc. London, n. ser., v. 4, p. 184 (1858). - C. eumeus var. a. C. a., W. F. Kirby, Cat. diurn. Lep., p. 119 (1871). c. a., G. F. L. Marshall \& Nicéville, Butt. Ind. Burm. Ceyl., v. 1. II, p. 314, fig. (? $C$. eumeus local-var.) (1883). - C.e. var. a., $\mathrm{O}$. Staudinger (\& Schatz), Exot. Schmett., v. 1, p. 202 (1887). - C. a., Swinhoe, Trans. Ent. Soc. London, p. 276 (1893). - F. Moore, Lep. Ind., v. 2, p. 208, t. 163, f. 1, $1 \mathrm{a}-\mathrm{c}\left(\sigma^{\prime}\right.$, $)$ (1895). $-\mathrm{E}$. Reuter, Acta

Assam,

Südost-

Tibet 
Soc. Sci. Fenn., v. 22, p. 110 (1896). - C. eumeus a., Fruhstorfer, Berlin. Ent. Zeit., v. 44, p. 51 (1899). - Id., Soc. Entom., v. 20 , p. $35(1905)$. - Faunis assamus, Stichel, Gen. Ins., fase. 36, p. 36 (1906). F. assama, Fruhstorfer: A. Seitz, Großschmett. Erde, v. 9, p. 406 (1911). - F. assamus, II. Stichel, Tierreich, Lief. 34, p. 102,119, f. 18 (\%) (1912). - Clerome eumeus „race" assama, IV. H. Evans, Journ. Bombay Nat. Hist. Soc., v. 21, p. 571 (1912). - C. e. a., id., l. c., v. 23, p. 598 (1915)., - Faunis e. a., id., 1. c., v. 29, p. 794 (1923). - Clerome a.. Antram, Butterfl. Ind., p. 117 , f. 242a (1924). - N. D. Riley \& Gabriel, Cat. Type Spec. Rhop. Brit. Mus., pars 1, p. 9 (1924). - Faunis e. a., W. H. Evans, Identif. Ind. Butterfl., p. 85 (1927).

aerope (Leech) Clerame a., Leech, Entomologist, China, v. 23, p. 31 (1890). - Faunis a., H. Stichel, Tonkin Gen. Ins., fasc. 36, p. 36 (1906). - Fruhstorfer: A. Seitz, Großschmett. Erde, v. 9, p. 406 (1911). - II. Stichel, Tierreich, Lief. 34, p. 102, 120 (1912).

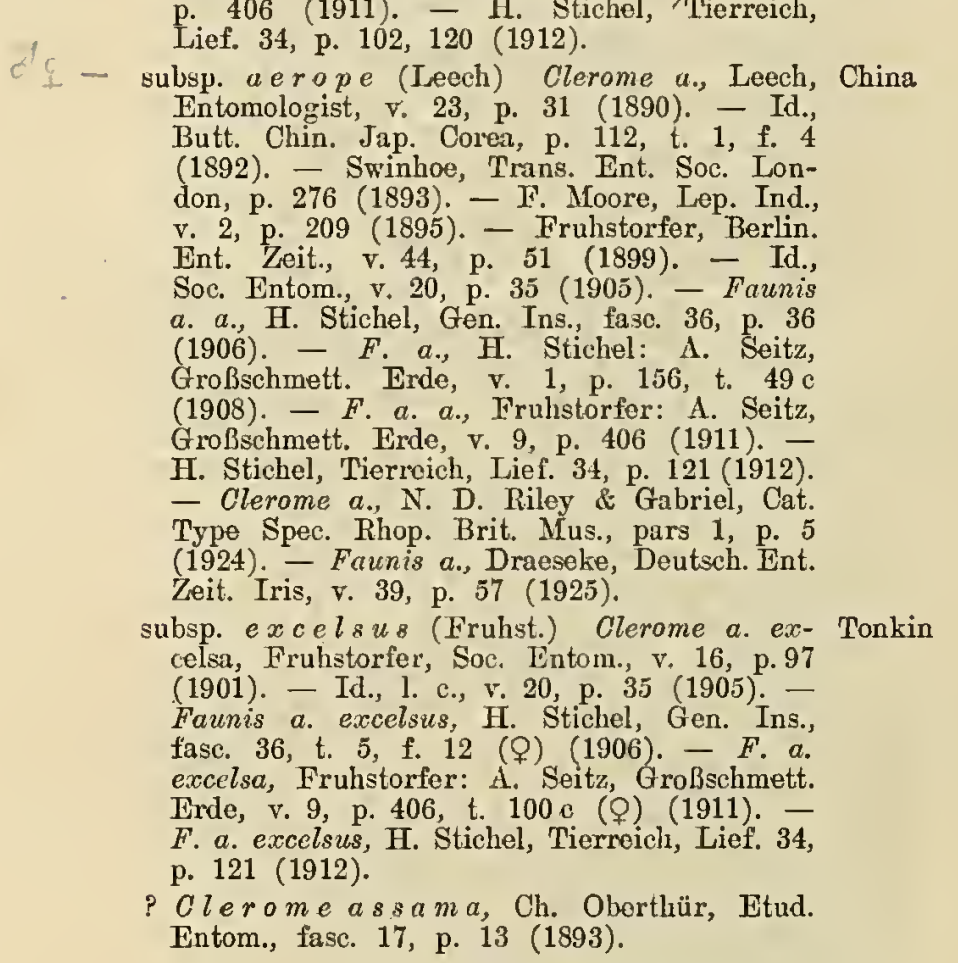

\section{Sectio Thaumafurgi}

H. Stichel, Gen. Ins., fasc. 36, p. 36 (1906). - Id., Tierreich, Lief. 34 , p. 122 (1912). 
faunula (Westw.) Thaumantis $f$., (E. Doubleday \&) Malakka, Westwood, Gen. Diurn. Lep., t. 54, f. 1; Siam, Clerome f., v. 2, p. 334 (1849 \& 51). - C. Cambodia. (Melanocyma) f., Westwood, Trans. Ent. Soc. London, n. ser., v. 4 , p. 186 , t. 21, f. 2 (क) (1858). - C. f., Herrich-Schäffer, Corr.Bl. Zool.-Min. Ver. Regensb., v. 19, p. 89 (1865). - W. F. Kirby, Cat. Diurn. Lep., p. 119 (1871). - Id., Entomologist, v. 10, p. 292 (1877). - Distant, Rhop. Ḿalay., p. 81, t. 8, f. 2 (1882). - Melanocyma f., G. F. L. Marshall \& Nicéville, Butt. Ind. Burm. Ceyl., v. 1. II, p. 315 (1883). - Clerome f., O. Staudinger (\& Schatz), Exot. Schmett., v. 1 t t. 65 ; p. 203 (1886 \& 87). - E. Haase, Corr.-Bl. Ent. Ver. Iris, v. 1, p. 311 (Organ. odor.) (1888). - Elwes (\& O. Möller'), Trans. Ent. Soc. London, p. 282 (1888). -

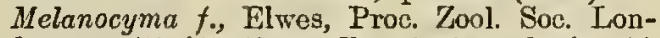
don, p. 271 (1891). - Faunis faunula (part.) F. f. f., H. Stichel, Gen. Ins., fase. 36, p. 36 (1906). - Fruhstorfer: A. Seitz, Großschmett. Erde, v. 9, p. 406, t. 100 a (1911). H. Stichel, Tierreich, Lief. 34, p. 102, 123 (1912). - $F$. f., $F$. faunala (!), Godfrey, Journ. Nat. Hist. Soc. Siam, v. 2, p. 123 (1916). - F. faunula, W. H. Evans, Journ. Bombay Nat. Hist. Soc., v. 29, p. 794, t. 15 , fig. ${ }^{\mathrm{E}} 2.3$ (1923). - Clerome $f ., \mathrm{N}$. D. Riley \& Gabriel, Cat. Type Spec. Rhop. Brit. Mus., pars 1, p. 21 (1924). - Faunis $f .$, W. II. Evans, Identif: Ind. Butterfl., p. 85 , t. 15 , fig. E 2. 3 (1927). - $F$. $f_{\text {. }} f_{.}$, Godfrey, Journ. Siam Soc. Nat. Hist. Suppl., v. 7, p. $258(1930)$.

faunuloides (Nicév.) Melanocyma f., Nicéville, Journ. OberBombay Nat. Hist. Soc., v. 9, p. 259, t. N, Burma fig. 2 (क) (1895). - F. Moore, Lep. Ind., V. 2, p. 210, t. 167 , f. 1, 1 a (1895). - E. Y. Watson, Journ. Bombay Nat. Hist. Soc., v. 10, p. 65̃2 (1897). - Faunis faunula (part.), $F$. faunula faunuloides, H. Stichel, Gen. Ins., fasc. 36 , p. 36 (1906). - Fruhstorfer: A. Soitz, Großschmett. Erde, v. 9, p. 406 (1911). - H. Stichel, Tierreich, Lief. 34 , p. 122, 123 (1912). - Melanocyma faunula "race" faunuloides, W. H. Evans, Journ. Bombay Nat. Hist. Soc., v. 21, p. 571 (1912). - Faunis $f$. $f .$, id., l. c., v. 29, p. 794 (non t. 15, fig. I 2. 3: $=F$. faunula) (1923). $F$. faunuloides, Tytler, 1. c., v. 31, p. 260 (spec. distinct.) (1926). - $F$. faunula faunuloides, W. H. Evans, Identif. Ind. Buttorfl., p. 85 (non t. 15, fig. E 2. $3:=F$. faunula) (1927). 


\section{Genus: Xanfhofaenia Westw.}

X. (Subgen.), Westwood, Trans. Ent. Soc. London, n. ser., v. 4, p. 187 (1858). - X., W. F. Kirby, Cat. Diurn. Lep.; p. 238i (1871). - S. H. Scudder, Proc. Amer. Ac. Sci, v. 10, p. 289 (Typ. $X . b u-$ siris) (1875). - A. G. Butler, Trans. Linn. Soc. I.ondon, ser. 2, Zool., v. 1, p. 538 (1879). - W. F. Kirby, Sci. Proc. R. Dublin Soc., n. ser., v. 2, p. 309 (1880). - Distant, Rhop. Malay., p. 69, 82 (1882). - G. F. L. Marshall \& Nicćville, Butt. Ind. Burm. Ceyl,, v. 1. II, p. 2\$2, 283 (1883). - Kheil, Rhop. Nias, p. 24l (1884). E. Haase, Corr.-Bl. Ent. Ver. Iris, v. 1, p. 104 (1886). - O. Staudinger (\& Schatz), Exot. Schmett., v. 1, p. 145 (1886). - L. Glaser, Cat. Etymol. Col. Ieep., p. 316 (1887). - Röber: O. Staudinger \& Schatz, Exot. Schmett., v. 2, t. 30 (Morphol.), p. 184, 188 (1888 \& 89). - Skertehly, Ann. Mag. Nat. Hist;, ser. 6, v. 4, p. 210 (1889). - B. Hagen, Tijuschr. Nederl. Aardr. Gen., ser. 2, v. 7, p. 201 (1890). - Holland, Proc. Boston Soc. Nat. Hist., v. 25, p. $60(1890)$. - F. Moore, Lep. Ind., v. 2, p. 216 (1895). - L. Martin, Deutsch. Ent. Zeit. Lep., v, 8, 1895, p. 255 (1896). - Nicéville \& L. Martin, Journ. Asiat. Soc. Bcngal, v. 64. II, 1895, p. 397 (1896). - H. Stichel, Deutsch. Ent. Zeit. Iris, v. 15, p. 59 (1902). - L. O. H. Young, Journ. Bombay Nat. Hist. Soc., v. 15, p. 297 (1903). - Shelford, Journ. Straits Branch Asiat. Soc., Nr. 41, p. 111 (1904). - Bingham, Faun. Brit. Ind., Butterfl., v. 1, p. 182, 185 (Typ. X. busiris) (1905). - II. Stichel, Gen. Ins., fasc. 36, p. 56, t. 2, f. 10 (Morphol.) (1906). - A. Pagenstecher, Geogr. Verbr. Schmett., p. 412, 413 (1909). - Fruhstorfer: A. Seitz, Großschmett. Erde, v. 9, p. 407 (1911). - H. Stichel, Tierreich, Lief. 34, p. 5, 224, f. 42 (Morphol.) (1912). - W. H. Evans, Journ. Bombay Nat. Hist. Soc., v. 21, p. 571 (1912). - Moulton, Journ. Straits Branch $\Lambda$ siat. Soc., Nr. 65, p. 18 (1913). - W. Rothschild, Nov. Zool, v. 23, p. 300 (1916). - Eecke, Zool. Merledeel. Leiden, v. 4, p. 83 (1918). - W. H. Evans, Journ. Bombay Nat. Hist. Soc., v. 29 , p. 793, 794, t. 15, E 1 (1923). - Id., Identif. Ind. Butterfl.. p. 84,85 , t. 15 , E 1 (1927). - A. Seitz, Ent. Rundschau, $\nabla .45$, p. 24 (1928).

Clerome (part.), Westwood, Trans. Ent. Soc. London, n. ser., จ. 4, p. 187 (1858). - C., ? laera, Hewitson, Exot. Butt., v. 4 (3), text. ad t. [1] Clerome (1862). - Herb! Druce, Proc. Zool. Soc. London, p. 341 (1873). - N. D. Riley \& Gabriel, Cat. Type Spec. Rhop. Brit. Mus., pars 1, p. 11 (1924).

busiris (Westw.) Clerome (Xanthotaenia) b., West- Burma, wood, Trans. Ent. Soc. London, n, ser, v. 4, Siamp. 187 (1858). - X. b., G. F. L. Marshall \& Nicéville, Butt. Ind. Burm. Ceyl., v. 1. II, p. 285 (1883). - H. Stichel, Gen. Ins., fasc. 36, p. 57 (1906). - Fruhstorfer: A. Seitr, Großschmett. Erde, v. 9, p. 407 (1911).H. Stichel, Tiorreich, Lief. 34, p. 226 (1912).

- subsp. busiris (Westw.) Clerome (Xanthotaenia) $b$., Westwood, ut antea (1858). C. b., ? Iaera b., Hewitson, Exot. Butt., v. 4 (3), text. ad t. [1] Clerome, p. [2] (? Nymphalidar. spec.) (1862). - Xanthotaenia b., W. F. Kirby, Cat. Diurn. Lep., p. 238 (1871). - F. Moore, Proc. Zool. Soc. London, p. 827 (1878). - A. G. Butler,

Malayische

Falbinsel,

Sumatra, Borneo und benachbarte Inseln

Süd-

Burma, Tenasserim,

Siam, Malayische Halbinsel, ? Java 
Trans. Linn. Soc. London, ser. 2, Zool., v. 1, p. 538 (1879). - Distant, Rhop. Malay., p. 82 (distr. part.), t. 5 , f. 7 (1882). - G. F. L. Marshall \& Nicéville, Butt. Ind., Burm. Ceyl., v. 1. II, p. 282, 285 (distr. part.), fig. (1883). - E. Haase, Corr.-Bl. Ent. Ver. Iris, v. 1, p. 104 (Organ. odor.) (1886). - O. Staudinger (\& Schatz), Exot. Schmett., v. 1 , t. 50, p. 145 (part.) (18S5 \& 86). L. Glaser, Cat. Etymol. Col. Lep., p. 316 (1887). - Elwes \& Nicéville, Journ. Asiat. Soc. Bengal, v. 55. II, p. 419 (1887). - Elwes (\& O. Möller), Trans. Ent. Soc. London, p. 282 (1888). - Elwes, Proc. Zool. Soc. London, p. 271 (1891). - B. Hagen, Berlin. Ent. Zeit., v. 37, p. 144 (1892). F. Moore, Lep. Ind., v. 2, p. 216 (part.), t. 166, f. $1,1 \mathrm{a}-\mathrm{c}\left(\sigma^{7}, \ell\right)(1895)$. - Bingham, Faun. Brit. Ind., Isep., v. 1, p. 185 (distr. part.) (1905). - X. b. b., H. Stichel, Gen. Ins., fasc. 36, p. 57 (1906). Fruhstorfer: A. Scitz, Großschmett. Erde, v. 9 , p. 407 (1911). - X. b. b. forma principalis, H. Stichel, Tierreich, Lief, 34, p. 226, 227 (cit. nonnull. except.) (1912). - W. H. Evans, Journ. Bombay Nat. Hist. Soc., v. 21, p. 571 (1912). - Ollenbach, 1. c., v. 26, p. 870 (not. biol.) (1918). - Id., I. c., v. 27 , p. 887 (1921). - W. H. Evans, 1. c., v. 29, p. 794 (sine synon.), t. 15, fig. E 1. 1 (1923). - Pendlebury, Journ. Feder. Malay Stat. Mus., 'v. 11, p. 35 (1923). Clerome b., N. D. Riley \& Gabriel, Cat. Type Spec. Rhop. Brit. Mus., pars 1, p. 11 (1924). - Xanthotaenia b., W. H. Evans, Identif. Ind. Butterfl., p. 85 (sine synon.), t. 15, fig. E 1. $1(1927)$ - $-X$. そ. b., Godfroy, Journ. Siam Soc. Nat. Hist., Suppl., v. 7, p. 258 (1930).

subsp. s a $d i j$ a Frulist. $X$. b. s., Fruhstorfer: Sumatra, A. Seitz, Großschmett. Erde, v. 9, p. 407 (1911). - $X . \cdot b$. b. forma s., H. Stichel, Tierreich, Lief. 34, p. $227(1912)$. - X. b. ? Bangka, 8., W. Rothschild, Journ. Feder. Malay Stat. Mus., v, 8, p. 153 (1920).

? Cleromebusiris (non Westwool), Weyenbergh, Pet. Nouv. Entom., Année 6, p. 404 (1874). - Xanthotaenia b., B. Hagen, Tijdschr. Nederl. Aardr. Gen., ser. 2, v. 7, p. 199 (1890). - L. Martin, Deutsch. Ent. Zeit. Lep., v. 8, 1895, p. 255 (1896). - Nicéville \& L. Martin, Journ. Asiat. Soc. Bengal, v. 64. II, 1895 , p. 397 (1896). ? X. b. b., Eecke, Zool. Mededecl. Leiden, v. 4 , p. 83 (1918).

subsp. obs cura Butl. X. o., A. G. Butler, Nias

Ent. Monthly Mag., v. 20, p. 54 (1883). -

Kheil, Rhop. Nias, p. 24 (1884) - E.

Haase, Corr.-Bl. Ent. Ver. Iris, v. 1, p. 104 
(Organ. odor.) (1886). - O. Staudinger (\& Schatz), Exot. Schmett., v. 1, p. 145 (? X. busiris forma loci) (1886). - X. $b$. o., H. Stichel, Gen. Ins., fasc. 36, p. 57 (1906). - Fruhstorfer: A. Seitz, GroBschmett. Erde, v. 9, p. 407, t. 100 b (1911). - H. Stichel, Tiermeich, Lief. 34, p. 228 (1912). - X. o., N. D. Riley \& Gabriel, Cat. Type Spoc. Rhop. Brit. Mus., pars 1, p. 40 (1924).

X. busiris, F. Moore, Lop. Ind., จ. 2, p. 216 (in cit.) (1895).

subsp. polychroma Hag. X. p., B. Hagen, MentaweiEntom. Nachr., v. 24, p. 201 (1898). - Id,, Inseln Abh. Senckenb. Naturf. Ges., v. 20, p. 331, t. 1 , f. 5 (1902). - Id., Bei liebenswürd. Wilden, Anhang, p. 201 (1902). - X. busiris p., H. Stichel, Gen. Ins., fasc. 36 , p. 57 (1906). - Fruhstorfer: A. Seitz, Großschmett. Erde, v. 9, p. 407 (1911). - H. Stichel, Tierreich, Lief. 34, p. 227, 228 (1912).

subsp. batuensis Rothsch. X. b. b., W. BatuRothschild, Nov. Zool., v. 23, p. 300 (1916). Inseln

subsp. $b u \operatorname{ra}$ Stich. $X$. busiris burra, H. Borneo Stichel, Gen. Ins., fasc. 36, p. 57 (1906). Fruhstorfer: A. Seitz, Großschmctt. Erde, v. 9, p. 407 (1911). - H. Stichel, Tierreich, Lief. 34, p. 227 (1912). - Moulton, Journ. Straits Branch Asiat. Soc., Nr. 65, p. 18 (1913).

X. busiris, Herb. Druce, Proc. Zool. Soc. London, p. 341 (1873). - O. Staudinger (\& Schatz), Exot. Schmett., v. 1, p. 145 (part.) (1886). - Distant \& Pryer, Ann. Mag. Nat. Hist., ser. 5, v. 19 , p. 51 (not. biol.) (1887). - Hanitsch, Journ. Straits Branch Asiat. Soc., Nr. 34, p. 83 (1900). 1

Shelford, 1. c., Nr. 41, p. 111 (1904).

\section{Tribus Taenaridi}

Taenares (Coitus), Jac. Hübner, Verz. Schmett., p. 52 (1818). Taenaridi ('Tribus) (part.), H. Stichel, Gen. Ins., fasc. 36, p. 26 (1906). - Id., Tierreich, Lief. 34, p. 79 (1912). - "Taenariden"; E. Werner, Kaiser Wilhelmsland, p. 170 (1911). - Taenarinae (Subfam.), Eecke, Nov. Guinea, v. 13, Zool., Livr. 1, p. 67 (1915). - Id., I. c., v. 15, Zool., p. 37 (1924).

\section{Genus Taenaris Jac. Hübn.}

T a enaris, Tenaris (err. typogr.), Jac. Hübner, Verz. Schmett., p. 52 (Taenares), Anzeiger, p. 5; p. 53 (1818 \& 27). - Taenaris, id., Index Exot. Lep., p. 4 (1821). - S. H. Scudder, Proc. Amer. Ac. Sci., v. 10, p. 274 (Typ. T. urania) (1875). - L. Glaser, Cat. Etymol. Col. Lep., p. 313 (spec. omiss.) (1887). - Fruhstorfer, Wien. Int. Zeit., v. 24, p. 57, 119, 126 (1905). - H. Stichel, Gen. 
Ins., fasc. 36, p. 37 (1906). - Fruhstorfer: A. Seitz, Großschmett. Ende, v. 9, p. 409 (1911). - H. Stichel, Tierreich, Lief. 34, p. 124, fig. 19 (Morphol.) (1912). - "Strand, Archiv f. Naturg. 1912. A. 11. p. 65. - Moulton, Joura. Straits Branch Asiat. Soc., Nr. 65, p. 17 (1913). - Strand, Int. Ent. Zeit., จ. 8, p. 104 (1914). - G. A. Waterhouse \& Lyell, Butterfl. Austral., p. 49 (Typ. T. urania) (1914). - Eecke, Nova Guinea, v. 13, Zool., Livr. 1, p. 67 (1915). - Talbot, Proc. Ent. Soc. London, p. LVI (1915). - Strand, Lep. Niepeltiana, pars 2, p. 11 (1916). - W. Rothschild, Nov. Zool., v. 23, p. 302 (1916). - Strand, Soc. Ent., v. 33, p. 12 (1918). Strand, Bull. Soc. Zool. France, 51, p. 397, 402 (1927). - A. Seitz, Ent. Rundschau, v. 45, p. 24 (1928).

T e $n$ ar $i s$, Herrich-Schäffer, Corr.-Bl. Zool.-Min. Vor. Regensb., v. 19 , p. 89 (1865). - A. G. Butler, Trans. Ent. Soc. London, p. 487 (1870). - W. F. Kirby, Cat. Diurn. Iep., p. 117, App., p. 646; Suppl., p. 714, App., p. 846 (1871 \& 77). - A. G. Butler, Proc. Zool. Soc. London, p. 279 (1874). - Id., Proc. Zool. Soc. London, p. 468 (1877). - IV. F. Kirby, Entomologist, v. 10, p. 292 (1877). - Kirsch, Mitt. Zool. Mus. Dresden, v. 1, p. 120 (1877). - F. Müller, Jena. Zeit. Naturw., v. 11, p. 102 (Physiol.) (1877). - Id., Kosmos, Leipzig, v. 1, p. 334 (ut antea) (1877). - F. D. Godman \& O. Salvin, Proc. Zool. Soc. London, p. 611 (1880). - IV. F. Kirby, Sci. Proc. R. Dublin Soc., ser. 2, v. 2, p. 300 (1880). - Chr. Aurivillius, Srensk. Vet. Akad. Handl., v. 19, Nr. 15, p. 42 (1882). - B. Gerhard, Berlin. Ent. Zeit., v. 27, p. 181 (distr. geogr.), (1883). - A. G. Butler, Ann. Mag. Nat. Hist., ser. 5, v. 11, p. 402, 411 (1883). - A. Pagenstecher, Jahrb. Nassau. Ver. Naturk., v. 37, p. 178 (1884). - Distant, Rhopal. Malay., p. 427 (1886). - E. Haase, Corr.-Bl. Ent. Ver. Iris, v. 1, p. 103, 310 (Organ. odor.) (1886 \& 88). - O. Staudinger (\& Schatz), Exot. Schmett., v. 1, p. 191 (1887). - A. Pagenstecher, Jahrb. Nassau. Ver. Naturk., v. 41 , p. 91 (1888). - Elwes (\& O. Möller), Trans. Ent. Soc. London, p. 282 (1888). - Goss, Proc. Ent. Soc. London, p. XI (1888). - Röber: O. Staudinger \& Schatz, Exot. Schmett., v. 2, t. 31 (Morphol); p. 18t, 187 (1888 \& 89). - Ribbc, Dcutsch. Ent. Zcit. Lep., v. 2, p. 201, 240 (1889). - W. F. Kirby, Ann. Mag. Nat. Hist., sor. 6, v. 3, p. 160 (1889). - P. C. 'I. Snellen, Tijdschr. Ent., v. 32, p. 389 (1889). - Röber, Tijdschr. Ent., v. 34, p. 309 (1891). - G. Semper: C. Semper, Rcis. Arch. Philipp., pars 2. V, Schmett., p. 331 (1892). - IV. F. Kirby: Hübner \& Geyer, Samml. Exot. Schmett., Neue Ausg., v. 3, p. 46 (1892). - Tryon: J. P. Thomson, N. Guinea, $\Delta$ pp. 3, p. 257 (1892). - W. F. Kirby, Handb. Lep. (Allens Natural. Libr.), v. 1, p. 198 (1894). - Grose Smith, Nov. Zool., v. 1, p. 358 (189t). - K. M. Heller, Ent. Nachr., v. 20, p. 369 (1894). - Grose Smith \& W. F. Kirby, Rhop. Exot., v. 2, 3, t. Tenaris 1 etc. $(1894-98)$. - O. Staudinger, Deutsch. Ent. Zeit. Lep., v. 6, p. 363 (1894). F. Moore, Lep. Ind., v. 2, p. 212 (1895). - Elera, Cat. Faun. Filippin., จ. 2, p. 271 (1895). - Fürbringer (\& A. Pagenstecher): Scmon, Forschungsieis. 5, Denkschr. Med.Naturw. Ges. Jena, v. 8, p. (78) 238 (1895). - E. Reuter, Acta Soc. Sci. Fenn., v. 22, p. 110 (1896). - L. Martin, Deutsch. Ent. Zeit. Lep., v. 8, 1915, p. 256 (1916). - Nicéville \& L. Martin, Joura. Asiat. Soc. Bengal, v. 64. II, 
1895 , p. 395 (1896). - B. Hagen, Jahrb. Nassau. Ver. Naturk., v. 50, p. 79 (1897). - A. Pagenstecher, Abh. Senckenb. Naturf. Ges., v. 23, p. 405 (1897). - Fruhstorfer, Berlin. Ent. Zeit., v. 41, p. 301 (1897). - Brunner-Wattenwyl, Betracht. Farbenpr. Ins., p. 6, 10 (1897). - B. Hagen, Jahrb. Nassau. Ver. Naturk., v. 50, p. 79 (1897). - Eimer (\& Fickert), Entstehg. d. Arten, pars 2, Orthogen., p. 236, 314, 328 (spec. omiss.) (1897). - Grose Smith, Nov. Zool., v. 4, p. 313 (1897). - Ribbe, Deutsch. Ent. Zeit. Lep., v. 11, p. 108 (variatio) (1898). - K. Jordan, Nov. Lool., v. 5, p. 390, t. 15, f. 63 (antenna) (1898). A. Pagenstecher, Koolorica, v. 27, p. 93 (1899). - Holland, Nov. Zool., v. 7, p. 62 (1900). - Hanitsch, Journ. Straits Branch Asiat. Soc., Nr, 34, p. 83 (1900). - W. F. Kirby: Hübner \& Geyer, Samml. Exot. Schmett., Neue Ausg., v. 3, p. 46 (1901). - Fruhstorfer, Deutsch. Ent. Zoit. Lep., v. 14, 1901, p. 330 (1902). - H. Stichel, Doutsch. Ent. Zeit. Iris, v. 15, p. 59 (1902). - Fruhstorfer, Ins.-Börse, v. 20 , p. 357 ; จ. 21 , p. 389 ; v. 22 , p. $36(1903,04,05) .-$ Shelford, Journ. Straits Branel Asiat. Soc., Nr. 41, p. 110 (1904). - Fruhstorfer, Soc. Ent., v. 19, p. 129, 138; v. 20, p. 35 (1904 \& 05). - Id., Ent. Zcit., v. 18, p. 118 (1904). A. Pagenstecher, Geogr. Verbr. Schmett., p. 413 (1909). Hauser, Verh. Zool.-Bot. Ges. Wien, v. 60, p. (38) (1910).Rebel, 1. c., v. 61, p. (28) (1911). - A. Pagenstecher, Abh. Senckenb. Naturf. Ges., v. 33, p. 411 (1911). — N. D. Riley \& Gabriel, Cat. Type Specim. Rlıop. Brit. Mus., pars 1, p. 5, 55 (1924). - Le Cort, Enc. Ent., ser. B. 3, Lep., v. 1, p. 141 (Morphol.) (1928).

II eliconius [Subgen.] (part.), Iinné, Syst. Nat., ed. 10. p. 466 (1758). - Id., l. c., ed. 12, v. 1 . II, p. 757 (1767). - Goeze, Ent. Beytr., v. 3. I, p. 89 (1779). [D. H. Schncider], Nomencl. Entomol., p. 34 (1785). Gmclin-Linné, Syst. Nat., ed. 13, v. 1. V, p. 2251 (1790). $A r g v s$ (part.), Scopoli, Introd. Hist. Nat., p. 432 (1777).

Da na us [Subgen.] (part.), Goeze, Entom. Beytr., v. 3. I, p. 211 (1779). - D. (Festivus) (part.), [D. H. Schneider], Nomencl. Fntom., p. 35 (1785). - D. [Subgen.] (part.), Gmelin-Limné, Syst. Nat., ed. 13, v. 1. V, p. 2277 otc. (1790). $N y m p h a l i s$ [Subgen.] (part.), Goeze, Entom. Beytr., v. 3. I, p. $294(1779)$.

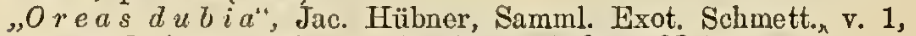
t. [84] (1806-19). - O., Oken, Lehrb. Naturg., v. 1, p. 740 (1815).

Morpho (part.), Latreille, Encs Méth., v. 9, p. 11, 435 (1819\& 24). - (Latreille \&) J. B. Godart, l. c., p. 445 (1824). - M., Guérin-Méneville, Voy. Coquille, Átl. Ins., t. 17 (1830). - M. (part.), Zinken-Sommer, Nov. Acta Ac. Leop., v. 15, p. 165, 167 (1831). - Verloren, Cat. Ins. Lep. Cramer, v. 1, p. 16 etc.; v. 2, p. 201 (1836). - $M$. (Drusilla), Westwood: Donovan, Epitome Ins. China, n. ed., p. 62 (1842).

Drusilla, Swainson, Zool. Illustr., v. 1, text. ad t. 11 (Typ. genaicus Prpilio jairus F.) (1820-21). - D. (Subgen.), J. O. Westwood: Donovan, Epitome Ins. China, n. ed., p. 62 (1842). - D., E.. Doubleday, List Lep. Brit. Mus., App., p. 29 (1848). - Chenu \& H. Lucas, Enc. Hist. Nat., Papill., p. 165 (1851). - (E. Doubleday \&) Westwood, Gen. Diurn. Lep., v. 2, p. 334 (1851). — White: 
Macgillivray, Voy. Rattlesnake, v. 2, App., p. 390 (1852). -

Ménétriés, Enum. Corp. Anim. Mus. Petropol., Lep., pars 1, p. 37; Suppl., p. 77 (1855 \& 57). - Horsfield \& F. Moore, Cat. Lep. Mus. E.-Ind. Comp., v. 1, p. 214 (1857). Westwood, Trans. Ent. Soc. London, n. sor., v. 4, p. 180 (1858). - C. \& R. Folder, Wien. Ent. Monatschr., v. 4, p. 209, $248(1860)$. - Snellen-Vollenhoren, Tijdschr. Entom., v. 3, p. 37 (1860). - Hewitson, Proc. Zool. Soc. London, p. 52 (1861). - Id., Exot. Butterfl., v. 3 (3), t. ¡2† Drusilla \& Hyantis (1862). - Snellen-Vollenhoven, Tijdschr. Entom., v. 6, p. 131 (1863). - Carus-Gerstäcker, Handb. Zool., v. 2, p. 232 (1863). - Herrich-Schäffer, Corr:-Bl. Zool.-Min. Ver. Regensb., v. 18, p. 112 (1864). A. G. Butler, Cat. Diurn. Lep. Fabr., p. 46 (1869). - W. F. Kirby, Trans, Ent. Soc. Iondon, p. $358(1869)$. - J. G. Ward, Ins. abroad, p. 591 (1874). - E. Martens, PreuB. Exp. Ost-Asion, v. 1, p. 290 (1875). - S. H. Scudder, Proc. Amer. Ac. Sci., v. 10, p. 159 (nom. praeoce.) (1875). F. D. Godman \& O. Salvin, Proc. Zool. Soc. London, p. 645 (1878). - Tid., 1. c., p. 157 (1879). - Ch. Oberthür, Ann. Mus. Civ. Stor. Nat. Genova, v. 12, p. 462 (1878). - Id., l. c., v. 15 , p. 508 (1880). - C. Crüger (sec. Kirby), Berlin. Ent. Zeit., v. 25, p. 111 (1881). - L. Glaser, Cat. Etymol. Col. Lep., p. 287 (1887). - P. C. T. Snellen, Tijdschr. Ent., v. 32, p. 389 (1889). - Tryon, 2. Ann. Rep. Admin. Brit. N. Guinea, App. 5, sep. p. 7 (1890). Drucilla (!), Id.: J. P. Thomson, Brit. N. Guinea, App. 3, p. 251 (1892). - Drusilla, N. D. Riley \& Gabriel, Cat, Type Specim. Rhop. Brit. Mus., pars 1, p. 17 etc. (1924).

II y a de s, Boisduval, Vor. Astrolabe, Ent., v. 1, p. 157 (1832). - Guérin-Mréneville, Voy. Coquille, Zool., v. 2. II, p. 272, 282 (post 1832). - Boisduval, Spec. Gén. Lép., v. 1, Expl. pl., p. 4, t. 13 (1836). - H., Haydes (1), Feisthamel, Voy. Favorite, v. 5. Suppl. Zool. Isep., p. 14; Tahl. Méth., p. 1 (1838). - Iyades, E. Blanchard, Hist. An. Artic. v. 3, p. $455(1840)$. - Lefebvre, Ann. Soc. Ent. France, v. 11, p. 30 (1842). - E. Blanchard, Hist. Ins., v. 2, p. 335 (1845). - Orbigny, Dict. Un. Hist. Nat., v. 12 (Ati. 2), p. 18 (1849) - C. \& R. Felder, Reise Novara, v. 2. II, p. 460 (1866). - S. II. Scudder, Proc. Amer. Ac. Sci., v. 10, p. 192 (Typ. bioculatus) (1875). - Wallace, Geogr. Distr. Anim., v. 1, p. 404 (1S76). - I. (Tenaris), Capronnier, Ann. Soc. Ent. Belg., v. 30, p. 6 (1886). - Tryon: J. P. Thomson, Brit. N. Guinea, App. 3, p. 251 (1892) $-H$. (Sectio), Fruhstorfer, Wien. Ent. Zeit., v. 24, p. 122 (1905). - H. Talbot, Proc. Ent. Soc. London, p. LVI (1915).

Morphotenaris, Fruhstorfer, Ent. Nachr., v. 19, p. 317 (1893). - Id., Berlin. Ent. Zeit., v. 39, p. 248 (1894).Id. Ent. Zeit. Stettin, v. 55, p. 127 (1891). - W. Rothschild, Nov. Zool., v. 3, p. 92 (1896). - Grose Smith (\& W. F. Kirby), Rhop. Exot., v. 3, t. Morphotenaris \& Hyantis; p. 9 (1898). - H. Stichel, Dcutsch. Ent. Zeit. Iris, v. 15, p. 59 (nota) (1902). - Bethune-Baker, Ann. Mag. Nat. Hist., ser. 8, v. 4, p. 183 (1909). - Lathy, Entomologist, v. 42, p. 272 (1909). - A. Pagenstecher, Geogr. Verbreit. Schmett., p. 413 (1909). - Fruhstorfer: A. Seitz, Großschmett. Erde, v. 9, p. 424 (1911). - E. Werner, Taisor Wilhelmsland, p. 170 (1911). - W. Rothschild (\& Durrant), Rep. Brit. Ornithol. Union. Exped. 
Dutch N. Guinea 2, Nr. 15, p. 19 (1915). - W. Rothschild, Nov. Zool., v. 23, p. 307 (1916). - Joicey \& Talbot, Bull. Hill Mus., v. 1, p. 328 (1922). - N. D. Riley \& Gabriel, Cat. Type Specim. Rhop. Brit. Mus., pars 1, p. 5 (1924).

Morphotaenaris, Fruhstorfer, Wien. Ent. Zeit., v. 24, p.122 (1905). - Joicey \& Talbot, Ann. Mag. Nat. Hist., ser. 8, v. 17, p. 73 (1916). - Talbot, Proc. Ent. Soc. London, p. LXXI, LXXII (1921). - Eecke, Nov. Guinea, v. 15 , Zool., p. 33 (1924).

Elymnotaenaris, Fruhstorfer: A. Seitz, Großschmett. Erde, จ. 9, p. 411 (1911).

\section{Sectio Xanfhorini}

H. Stichel, Gen. Ins., fasc. 36, p. 39 (1906). - Id., Tierreich, Lief. 34, p. 143 (1912).

\section{Cohors a. Horsfieldiiformes}

H. Stichel, Gen. Ins., fasc. 36, p. 39 (1906). - Id., Tierreich, Lief. 34 , p. 143 (1912).

Horsfleldii (Swains.) Drusilla horsfieldii, Swainson, Malayische Zool. Illustr., ser. 1, v. 1, Index $(1820-21)$. Halbinsel, - Taenoris horsfieldi, Fruhstorfer, Wien. Ent. Zeit., v. 24, p. 126 (1905). - T. horsfieldii, H. Stichel, Gen. Ins., fasc. 36 , p. 39 (1906). - T. horsfieldi, Fruhstorfer: A. Seitz, Großschmett. Erde, v. 9, p. 410 (1911). - T. horsfieldii, H. Stichel, Tierreich, Lief. 34 , p. $143(1912)$.

- subsp. II orsfieldii (Swains.) Drusilla horsfeildii (laps., corr. in Addend.: Horsfeild $=$ Horsfield), $D$. horsfieldii, Swainson, Zool. Mlustr., ser. 1, t. 11; Index (1820-21). D. horsfeildii, Thon, Ent. Archiv., v. 1, p. 90, t. 4, f. 4 (sec. Swains.) (1828). - Hyades horsfieldii, Boisduval, Spec. Gén. Lép., v. 1, Expl. pl., p. 4, t. 13 (9 B), f. 1 (1836). - Drusitha h., E. Doubleday, List Lep. Brit. Mus., App., p. 29 (1818). - Hyades $h .$, Orbigny, Dict. Un. Hist. Nat., $\nabla$. 12 (Atl. 2), p. 18, t. 7, f. 2 (1849). - Drusilla $h .$, E. Doubleday (\& Westwood), Gen. Diurn. Lep., t. 54, f. 4 (1849). Westwood, v. 2, p. 335 (1851). - Chenu \& H. Lucas, Enc. Hist. Nat., Papill., p. 165 , f. 277, p. 166 (1851). - D. horsfieldi, Horsfield \& F. Moore, Cat. Lep. Mus. E.-Ind. Comp., v. 1, p. 214 (1857). - D. horsfieldii, Ménétriés, Enum. Corp. Anim. Mus. Petropol., Suppl., p. 77 (1857). - Tenaris $h$, /Westwood, Trans, Ent. Soc. London, n. ser., v. 4, p. 181 (1858). - Herrich-Schäffer, Corr.-Bl. Zool.-Min. Ver, Regensb., v. 19, p. 89 (1865). - W. F. Kirby, Cat. Diurn. Lep., p. 117 (1871). - ? Hyades (Tenaris) h., Capronnier, Ann. Soc. Ent. Belg., v. 30, p. 6 (determin. falsa, conf. $T$. onolaus honrathi)

Sunda

Inseln,

Philippinen

Java 
(1886). - T. horsfieldii, O. Staudinger (\& Schatz), Exot. Schmett., v. 1, p. 192, 195 (1887). - F. Moore, Lep. Ind., v. 2, p. 212 (1895). - T. horsfieldi, Fruhstorfer, Berlin. Ent. Zeit., v. 41, 1896, p. 301 (1897). A. Pagenstecher, Abh. Senckenb. Naturf. Ges., v. 23, p. 405 (1897). - Taenaris h. h., Fruhstorfer, Wien. Ent. Zoit., v. 24, p. 71, 120 (1905). - T. horsfieldii .h., Stichel, Gen. Ins., fasc. 36, p. 39 (1906). T. horsfieldi h., Fruhstorfer: A. Seitz, Großschmett. Erde, v. 9, p. 410 (1911). - T. horsfieldii $h$. forma principalis, H. Stichel, Tierreich, Lief. 34, p. 145 (1912). - Drusilla h., N. D. Rilcy \& Gabriel, Cat. Type Spec. Rhop. Brit. Mus., pars 1, p. 25 (1924).

Morpho urania (non Papilio $u$. Linné), Zinken-Sommer, Nova Acta Ac. Leop., v. 15, p. 167 (cit. part.) (1831). - Drusilla u. var., Hewitson, Proc. Zool. Soc., p. 52 (1861). forma mor os a Stich. Taenaris $h$. $h$. forma $m$. H. Stichel, Gen. Ins., fusc. 36, p. 39 (1906). - T. horsfieldi $h$. ,Form" m., Fruhstorfer: A. Seitz, Großschmett. Erde, v. 9, p. 410 (1911). - T. horsfieldii $h$. forma $m$., H. Stichel, Tierroich, Lief. 34, p. 145, f. 20 (1912).

forma opulenta Fruhst. $T$. horsfieldi $h$. Typ.: Java

„Torm" o., Fruhstorfer: A. Seitz, Großschmett. Frde, v. 9, p. 410 (1911). - T.

horsfieldii $h$. forma a., II. Stichel, Tierreich, Lief. 34, p. 146 (1912).

subsp. Birchi (Dist.) Tenaris b., Distant, Malayische Ann. Mag. Nat. Hist., ser. 5, v. 12, p. 241 Halbinsel (1883). - Id., Rhopal. Malay., p. 428, t. 39, f. 7 (1886). - T. horsfieldii var. b., 0 . Staudinge. (\& Schatz), Exot. Schmett., v. 1, p. 192, 195 (distr. part.) (1887), - T. b., Elwes (\& O. Möller), Trans. Ent. Soc. London, ก. 282 (1888). - O. Staudinger, Deutsch. Ent. Zeit. Trep., v. 2, p. 43 (1889). - T. birchii, F. Mooro, Lep. Ind., v. 2, p. 212 (1895). - T. birchi, I. Martin, Deutsch. Ent. Zeit. Lep., v. 8, 1895, p. 256 (1896). Niceville \& T. Martin, Journ. Asiat. Soc. Bengal, v. 64. IT, 1895, p. 395 (1896). Taenaris horsfieldi b., Fruhstorfer, Wien. Ent. Zoit., v. 24, p. 71, 120 (1905). - T. horsfieldii b., II. Stichel, Gen. Ins., fasc. 36, p. 40 (1906). - T. horsfieldi $b$., Fruhstorfer: A. Seitz, Großschmett. Fide, จ. 9, p. 411 (1911). - T. horsficldii b., H. Stichel, Tierreich, Lief. 34, p. 146 (1912).

subsp. o c culta (Gr. Smitli) Tenaris o., Borneo

Grose Smith, Ann. Mag. Nat. Hist., ser. 6, v. 3, p. 316 (1889). - Grose Smith \& W. F. Kirby, Rhop. Exot., v. 2, t.Tenaris 1, f. 5, 6, p. 2 (1894). - F. Moore, Top. Ind., v.

2, p. 212 (1895). - Hanitsch, Journ. Straits

West-Java (Soekaboemi) 
Branch Asiat. Soc., Nr. 34, p. 83 (1900). Shelford, l. c., Nr. 41, p. 110 (1904). Taenaris horsfieldi o., Fruhstorfer, Wien. Ent. Zeit., v. 24, p. 71, 120 (1905). - T. horsfieldii o., H. Stichel, Gen. Ins., fasc. 36 , p. 40 (1906). - T. horsfieldi o., Fruhstorfer: A. Seitz, Großschmett. Frde, v. 9, p. 411 (1911). - T. horsfieldii o., H. Stichel, Tierreich, Lief. 34, p. 146 (1912). - Moulton, Journ. Straits Branch Asiat. Soc., Nr. 65, p. 17 (1913).

subsp. Plat en $i$ (O. Stauding.) Tenaris $h . p$.,

O. Staudinger, Deutsch. Ent. Zeit. Lep., v. 2, p. 43 (1889). - T. p., F. Moore, Lep. Ind., v. 2, p. $212(1895)$. - Taenaris horsfieldii p., H. Stichel, Gen. Ins., fasc. 36, p. 40 (1906). - T. horsfielni p., Fruhstorfer: A. Seitz, Großschmett. Frde, v. 9, p. 411, t. $100 \mathrm{c}$ (1911). - T. horsfieldii p., H. Stichel, Tierreich, Lief. 34, p. 147 (1912).

Tenaris horsfieldi, G. Semper: O. Semper, Reis. Arch. Philippin., pars 2. V, Schmett., p. 331 (1892). - ? Elera, Cat. Faun. Filipin., v. 2, p. 271 (1895).

\section{Cohors b. Uraniiformes}

H. Stichel, Gen. Ins., fasc. 36, p. 40 (1906). - Id., Tierreich, Lief. 34, p. 147 (1912).

urania (L.) Papilio (Heliconius) u., Linné, Syst. Molukken, Nat., ed. 10, p. 466 (1758). - Taenaris $u$. , Ulinsser Fruhstorfer, Wien. Ent. Zeit., v. 24, p. 126 (1905). - H. Stichel, Gen. Ins., fasc. 36, p. 148 (1906). - Truhstorfer: A. Seitz, Großschmett. Erde, v. 9, p. 411 (1911). - H. Stichel, Tierreich, Lief. 34. p. 148 (1912).

- subsp. urania (L.) Pap.lio (Heliconius) u., Amboina, Linné, ut antea (1758). - P. u., id., Mus. Uliasser Ludov. Ulr., p. 225 (1764). - P. (Heliconius) u., Linné-Houttuyn, Natuurl. Hist., v. 1. XI, p. 231 (non t. 88, f. 2: = Heliconius spec.) (1766-69). - Linné, Syst. Nat., ed. 12, v. 1. II, p. $756(1767)$. - P. u., P. S. L. Müller, Naturs. Linué, v. 5. I, p. 584 (1774). - J. C. Fabricius, Syst. Ent., p. 461 (1775). - (Gmelin \&) Christman, Onomatol. Ired., v. 6, p. $159(1775)$. $-A r$ gus u., Scopoli, Introd. Hist. Nat., p. 432 (1777). - Papilio (Heliconius) u., Goeze, Entom. Beytr., v. 3. II, p. 99 (1779). P. vrania, J. C. Fabricius, Spec. Ins., v. 2, p. 29 (1781) - P. (Heliconius) u., [D. H. Schneider $]$, Nomencl. Entom., p. 34 (1785). $-P$. u., J. C. Fabricius, Mant. Ins., v. 2, p. 14 (1787). - P. -, Zschach, M[us. Leskean., p. 89, Nr. 47 (sec. Kirby, 1869) (1788). P. u., (Jahlonsky \&) Herbst, Naturs. Ins., Schmett., v. 4, p. $169(1790)$. - P. (Heliconius) u., Grelin-Linné, Syst. Nat., ed. 13, 
p. 2251 (1790). - P. u., Jung, Alphab. Verz. Schmett., v. 2, p. 276 (1792). - J. O. Fabricius, Ent. Syst., v. 3. I, p. 166 (1793). - Latham \& H. Davis: J. A. Forster, Zool. Index, ed. 2. pars 3, p. 24 (1795). - Turton, Gen. Syst. Nat. Linné, v. 3. II, p. 46 (1806). - Drusilla u., Zinken-Sommer, Nova Acta Ac. Leop., v. 15. II, p. 167 (part. in cit.) (1831). - Chenu \& H. Lucas, Enc. Hist. Nat., Papill., p. 166 (1851). - (E. Doubleday \&) Westwood, Gen. Diurn. Lep., v. 2. p. 335 (part.) (1851). - Ménétriés, Finum. Corp. Anim. Mus. Petropol., pars 1, p. 37 (1855). - Westwood, Trans. Ent. Soc. London, ser. 2, v. 4, p. 180 (synon. part.) (1858). - Tenaris u., Merrich-Schäffer, Corr.-Bl. Zool.-Min. Vcr. Regensb., v. 19, p. 89 (part.) (1865). - Drusilla u., A. G. Butler, Cat. Diurn. Lep. Fabr., p. 44 (1869). - Tenaris u., W. F. Kirby, Cat. Diurn. Lep., p. 117; Suppl., p. 714, App. p. 846 (1871 \& 77). - ? Drusilla u., Ch. Oberthür, Ann. Civ. Stor. Nat. Genova, v. "15, p. 508 (1880). - Tenaris u., W. F. Kirby, Sci. Proc. R. Dublin Sac., ser. 2, v. 2, p. 300 (1880). Chr. Aurivillius, Svenska Vet. Ak. Handl., ser. 2, v. 19, Nr. 5, p. 43 (1852). - A. Pagenstecher, Jahrb. Nassau. Ver. Naturk., v. 37, p. $178(1884)$. - O. Staudinger (\& Schatz), Exot. Schmett., v. 1, p. 192, 193 (part.) (1887). - A. Pagenstocher, Jahrb. Nassau. Ver. Naturk., v. 41, p. 91 (1888). Ribbe, Deutsch. Ent. Zeit. Lep., v. 2, p. 240 (1889). - Fürbringer (\& A. Pagenstecher): Semon, Zool. Forschungsreis. 5, Denkschr. Med.-Naturw. Ges. Jena, v. 8, p. (78) 238 (1895). - A. Pagenstecher, Abh. Senckenb. Naturf. Ges., v. 23, p. 405 (part.) (1897).Brunner-Wattenwyl, Betr. Farbenpr. Ins., p. 6, 10, t. 5, f. 53 (1897). - Taenaris $u$. u., Fruhstorfer, Wien. Ent. Zeit., v. 24, p. 70 (distr. part.) (1905). - H. Stichel, Gen. Ins., fasc. 36 , p. 40 (patria corrig.) (1906). - Fruhstorfor: A. Seitz, Großschmett. Erde, v. 9, p. 411 (1911). - T. u. u. forma principalis, H. Stichel, Tierroich, Lief. 34, p. 149 (distr. part.) f. 21 (O) (1912).

"Cla s sia e" (non Pap. c. Linne, 1758), Clerck, Icon. Ins., sect. 2 , t. 29 , ordo 3 , f. $[5,6]$; Register (1759-64).

marinus. (Gmelin) Papilio (Danaus) m., Gmelin-Iinné, Syst. Nat., éd. 13, p. 2289 (part.) (1790) (vide: W. F. Kirby, Trans. Ent. Soc. London, 1869, p. 358: recto murinus).

$n$ y $\boldsymbol{s} a$ Hübn. Tenaris n., Jac. Hübner, Verz. Schmett., p. 53 [1818].

"J airus", Papilio $j$. (non Cramer, 1775), P. Cramer, Pap. Exot., v. 2, p. 134, 135, t. 
185, f. A ( $\left.\sigma^{7}\right)$, f. B, C (Q); p. 149 (1777). $-P$. (Nymphalis) j., Goeze, Ent. Beytr., v. 3. I, p. 294 (part.) (1779). - P. iairus, J. C. Fabricius, Spee. Ins., v. 2, p. 63 (part.) (1781). - P. jairus, id., Syst. Ent., v. 3. I, p. 54 (part.) (1793). - Donovan, Epitome Ins. China, t. 37 (in text. cit. part., patria falsa) (1798). - Morpho $j$. , (Latreille \&) J. B. Godart, Enc. Méth., v. 9, p. 445 (part. in cit.) (1824). - ? Hyades j., Boisduval, Voy. Astrolabe, Faune Entom., pars 1, p. 157 (part.) (1832). - Morpho j., Verloren, Cat. Ins. Lep. Oramer, v. 1, p. 76; v. 2, p. 201 (part.) (1837). - Hyades j., R. Blanchard, Hist. Anim. Artic., v. "3, p. 455 (part.) (1840). - Morpho (Drusilla') j., Westwood: Donovan, Epilome Ins. China, n. ed., p. 62, t. 33 (Morpho j.) (1842). Tenaris selene var. jaira, A. G. Butler, Trans. Ent. Soc. London, p. 487 (1870). T. jairus, A. Pagenstecher, Jahrb. Nassau. Ver. Naturk., v. 37, p. 178 (1884).

forma jairus (Cram.) „Jairus", Papilio j., Typ.: „Indes P. Cramer, Pap. Exot., v. 1, p. 9, t. 6, f. A, B; p. $153(1775 \& 76) . \quad$ \&. j., J. O. Fabricius, Syst. Ent, p. 461 (1775). $-P$. iairus, id., Gen. Ins., p. 258 (1777), $-P$. jairus, Goeze, Entom. Beytr., v. 3. II, p. 211, 294 (1779). - P. iairus, J. O. Fabricius, Spec. Ins., v. 2, p. 63 (part.) (1781). $P$. iairis, C. Stoll, Essai Syst. Lep.: P. Cramer, Lep. Exot., v. 4, Append., p. 8 (1782). - P. (Danaus Festivus) jairus, [D. H. Schneider], Nomencl. Entom., p. 35 (1785). - P. iairus, Herbst: Borowski, Gemeinnütz. Naturg. Tierr., v. 7. I, p. 51, t. $44 \mathrm{I}, \mathrm{f} .1$ (1786). - P. iairus, J. C. Fabricius, Mant. Ins., v. 2, p. 30 (1787). - P. (Danaus) $j$., Gmelin-Linné, Syst. Nat., ed. 13, v. 1. $\nabla$, p. $2277(1790) .-P . j$. (P. amboin.) $7 P P . j$. (P. Ind.), Jung, Alphal. Verz. Schmett: v. 1, p. 279 (1791). - J. C. Fabricius, Ent. Syst. จ. 3. I, p. 54 (part.) (1793). - Latham \& H. Davis: J. A. Forster, Zool. Index, ed. 2, pars 3, p. 25 (1795). - P. iairus, (Jablonsky \&) Herbst, Naturs. Ins., Schmett., v. 8, p. 229 , t. 218, f. $1-3(1796)$. $-P$. jairus, Tabl. Encycl. Méth., v. 18, t. 30 , f. 30,30 bis (1797). - Donovan, Epitome Ins. China, text. ad t. [33] (part.) (non fig.: = T. urania u.) (1798). - G. Shaw, Natural. Miscell., v. 15, t. 632; index (1804). Turton, Gen. Syst. Nat. Linné, v. 3. II, p. 60 (1806). - P. iairus, Gravenhorst, Vergleich. Uebers. Zool. Syst., p. 313 (1807). - Tenaris jaira, Jac. Hübner, Verz. Sehmett., p. 53 [1818]. - Taenaris j., id., Index Exot. Lep., p. 4 (1821). - Morpho jairus, (Latreille \&) J. B. Godart, Enc. Méth., v. 9, p. 
445 (cit. part.) (1824). - Hyades $j$. , Boisduval, Voy. Astrolabe, Faune Entom., pars 1, p. 157 (part.) (1832). - Morpho j., Verloren, Cat. Ins. Lep. Cramer, v. 1, p. 16; v. 2, p. 201 (part.) (1837). - ? Hyades j., Haydes $j$., Feisthamel, Voy. Favorite, v. 5, Suppl., Zool., Lép. 14 (variatio); Tabl. Méth., p. 1 (1838). - Hyades j., E. Blanchard, Hist. An. Artic., v. 3, p. 455 (sine cit.) (1840). - ? Lofebrre, Ann. Soc. Ent. France, v. 11, p. 30, t. 3, f. 5 (Morphol.) (1842). - Drusilla urania "race" Pap. jairus, A. G. Butlor, Cat. Diurn. Lop. Fabr., p. 44 (1869). - T. urania var. a. Pap. jairus, W. F. Kirby, Cat. Diurn. Iep., p. 117 (cit. part.) (1871). - T. urania ab. j., T. $j .$, O. Staudinger (\& Schatz), Exot. Schmett., v. 1, p. 192, 193 (1887). - T. u. j., H. Stichel, Gen. Ins., fasc, 36, p. 40 (distr. part.) (1906). - T. u. jaira, Fruhstorfer: A. Seitz, Großschmett. Erde, v. 9, p. 411 (1911). - T. u. $u$. forma jairus, H. Stichel, Tierreich, Lief. 34 , p. 150 (1912).

marinus (Gmelin) Papilio m., GmelinLinné, Syst. Nat., ed. 13, v. 1. V, p. 2289 (cit. part.) (1790).

Drusilla urania (non'Pap. u. Linné), (E. Doubleday \&) Wostwood, Gen. Diurn. Lep., v. 2, p. 335 (part.); Addit., p. 533 (1851 \& 52). - Westwood, Trans. Ent. Soc. London, ser. 2 , v. 4, p. 180 (part.) (1858). - Tenaris u., Herrich-Schäffer, Corr.-BI. Zool.-Min. Ver. Regensb., v. 19, p. 89 (part.) (1865). Drusilla u., W. F. Kirby, Trans. Ent. Soc. London, p. 358 (1869). - Tenaris u., $\mathrm{O}$. Staudinger (\& Schatz), Exot. Schmett., v. 1, p. 193 (part.) (1887). - A. Pagenstecher, Jahrb. Nassau. Vor. Naturk., v. 41, p. 91 (1888). - Id., Abh. Senckenb. Naturf. Ges., v. 23, p. 405 (sino cit.) (1897).

- forma nox (Kirb.) Tenaris n., W. F. Kir- Typ.: ? by: Hübner \& Geyer, Samml. Exot. Schmett., Neue Ausg., v. 3, p. 47 (1901). - T. urania jairus forma n., H. Stichel, Gon. Ins., fasc. 36, p. 41 (1906). - Taenaris u. n., Fruhstorfer: A. Seitz, Großschmett. Erde, v. 9, p. 411 (1911). - T. u. $u$. forma n., $H$. Stichel, Tierreich, Lief. 34 , p. 148 (1912).

Oreas dubia iaira (non Pap. jairus Cramer), Jac. Hübner, Samml. Exot. Sehmett., จ. 1, t. [84], f. 1, $2(1806-19)$. - Tenaris selene var. jaira, A. G. Butler, Trans. Ent. Soc. London, p. 487 (1870). - T. s. var. a. Oreas dubia jaira, W. F. Kirby, Cat. Diurn. Lep., App., p. 646 (1871). - T. urania ab. $j$., O. Staudinger (\& Schatz), Exot. Schmett., v. 1, p. 192, 193 (1887). - T. u. var. jaira, Ribbe, Deutsch. Ent. Zoit. Lep., v. 2, p. 240 (1889). - Oreas Dubia j., W. F. Kirby: 
Hübner \& Geyer, Samml. Exot. Schmett., v. 1, t. 84 , f. 1,2 (1894-95).

subsp. pand e mos Fruhst. Taenaris urania p., Fruhstorfer: A. Seitz, Großschmett. Erde, v. 9, p. 411 (1911). - T. u. u. forma p., H. Stichel, Tierreich, Lief. 34, p. I50 (1912). - T. u. p., W. Rothschild, Nov. Zool., v. 22, p. 124 (1915).

Tenaris urania (non Pap. u. Linné), Ribbe, Deutsch. Ent. Zeit. Lep., v. 2, p. 240 (1899). - Röber, Tijdschr. Entom., v. 34, p. 309 (1891). - Tuenaris u. u., H. Stichel, Gen. Ins., fasc. 36, p. 40 (part.) (1906). - Id., Tierreich, Lief. 34, p. 149 (part.) (1912).

forma $d u p l e x$ Stich. T. urania $u$. forma $d$., Typ.: Ceram H. Stichel, Gen. Ins., fasc. 36, p. 40 (1906).

- T. u. pandemos forma d., Fruhstorfer: A. Seitz, Großschmett. Erde, v. 9, p. 411 (1911). - T. u. u. forma d., H. Stichel, Tierreich, Lief. 34, p. 150 (1912).

subsp. Hollandi (Fruhst.) Tenaris urania Buru $h$., Fruhstorfer, Ent. Zeit. Guben, v. 18, p. 118 (1904). - Taenaris $u$. $h$, id., Wien. Ent. Zeit., v. 24, p. 70 (1905). - H. Stichel, Gen. Ins, fasc. 36 , p. 41 (1906). Fruhstorfer: A. Seitz, Großschmett. Erde, v. 9. p. 411, t. 100 c, p. 1111 (1911 \& 27).HI. Stichel, Tierreich, Lief. 34, p. 151 (1912). - Eecke, Treubia, v. 7, p. 359 (fig. in Seitz, t. $100 \mathrm{c}$ non typ.) (1929).

Te naris urania, Holland, Nov. Zool., v. 7 , p. 62 (1900).

diana (Butl.) Tenaris d., A. G. Butler, Trans. Ent. NordSoc. London, p. 487 (1870). - Taenaris d., Fruhstorfer, Wien. Ent. Zeit., v. 24, p. 126 (1905). - H. S'ishel, Gen. Ins., fasc. 36, p. 41 (1906). - Fruhstorfer: A. Seitz, Großschmett. Erde, v. 9, p. 411 (1911). - H. Stichel, Tierreich, Lief. 34, p. 151 (1912).

subsp. diana (Butl.) Tenaris d., A. G. Ternate, Butler, ut antea (1870). - W. F. Kirby, Batjan Cat. Diurn. Lrop., App., p. 646 (1871). - O. Staudinger (\& Schatz), Exot. Schmett., v. 1, p. 192, 193 (distr. part.) (1887). - A. Pagenstecher, Abh. Senckenb. Naturf. Ges., v. 23, p. 405 (1897). - Taenaris d. d., Fruhstorfer, Wien. Ent. Zeit., v. 24, p. 122 (1905). - H. Stichel, Gen. Ins., fasc. 36, p. 41, t. 5. f. 13 (1906). - Fruhstorfer: A. Seitz, Großschmett. Errle, v. 9, p. 411 (1911). T. d. d. forma principalis, H. Stichel, Tierreich, Lief. 34, p. 152 (1912). - Tenaris d., N. D. Riley \& Gabriel, Cat. Type Specim.

Rhop. Brit, Mus., pars 1, p. 17 (1924).
- subsp. di i de ma (Truhst.) Tenaris diana diadema, Fruhstorfer, Ins.-Börse, v. 20, p. 357 (1903). - Taenaris d. d., id., Wien. Ent. Zeit., v. 24, p. 122 (1905). - H. Stichel, 
Gen. Ins., fasc. 36, p. 41 (1906). - Fruhstorfer: A. Seitz, Großschmett. Erde, v. 9, p. 411, t. $100 \mathrm{c}$ (1911). - H. Stichel, Tierreich, Lief. 34, p. 152 (1912).

subsp. bisae Rothsch. T. d. b., W. Rothschild, Nov. Zool., v. 23, p. 302 (1916).

Pulo Bisa

(nöndl. Obi)

subsp. le to Fruhst. T. d. l., Fruhstorfer, Halmahera

Wien. Ent. Zeit., v. 24, p. 73,122 (1905). - (=Djilolo)

H. Stichel, Gen. Ins., fasc. 36, p. 41 (1906).

- Fruhstorfer: A. Seitz, Grofschmett. Erde, v. 9, p. 411 (1911). - T. d. l. forma principalis, H. Stichel, Tierreich, Lief. 34 , p. 153 (1912).

forma aberrans (Stauding.) Tenaris d. Typ.:

ab. a., O. Staudinger ( $\&$ Schatz), Exot. Halmahera

Schmett., v. 1, p. 192,193 (1887). - Taenaris d. leto ab. a., Fruhstorfer, Wien. Ent. Zeit., v. 24 , p. 122 (1905). - T. $d$. $d$. forma a., H. Stichel, Gen. Ins., tasc. 36, p. 41 (1906). - T. d. d. (a.), Truhstorfer: A. Seitz, Großschmett. Ende, v. 9, p. 411 (1911). - T. d. d. forma a., H. Stichel, Tierreich, Lief. 34 , p. 152 (1912).

forma ge m mat a Fruhst. T. d. l. (g.), Fruh- Typ.: storfer: A. Soitz, Großschmett. Lirde, v. 9, Halmahera p. 411 (1911). - T. d. l. forma g., H. Stichel, Tierreich, Lief. 34, p. 153 (1912).

Butleri (Oberth.) Drusilla b., Ch. Oberthür, Ann. Yule- (Lolo-) Mus. Civ. Stor. Natural Genova, v. 15, p. Insel, 512 (1880). - Kirsch, Berlin. Ent. Zeit., v. südl. 27, p. 304 (1883). - Tenaris b., O. Stau- Neu-Guinea diuger (\& Schatz), Exot. Schmett., v. 1, t. 64; p. 193, 201 (1886 \& 87). - Tryon: J. P. Thomson, Brit. N. Guinea, App. 3, p. 258 (1892). - Taenaris b., Fruhstorfer, Wien. Ent. Zeit., v. 24, p. 124 (1905). H. Stichel, Gen. Ins., fasc. 36, p. 41 (1906). - Fruhstorfer: A. Seitz, Großschmett. Erde, v. 9, p. 421 (1911). - H. Stichel, Tierreich, Lief. 34, p. 153 (1912). - W. Rothschild \& K. Jordan, Nov. Zool., v. 12, p. 454 (nota) (1905).

pleiops (Kirsch) Drusilla p., Kirsch, Berlin. Ent. Zeit., v. 27, p. 164 (corr. p. 304, vide supra) (1883).

\section{Cohors c. Domifilliformes}

H. Stichel, Gen. Ins., fasc. 36, p. 41 (1906). - Id., Tierreich, Lief. 34 , p. 154 (1912).

domitilla (Hew.) Drusilla d., Hewitson, Proc. Zool. NordSoc. London, p. 51 (1861). - Taenaris d., Molukken Fruhstorfer, Wien. Ent. Zieit, v. 24, p. 126 (1905). - H. Stichel, Gen. Ins., fasc. 36, p. 41 (1906). - Fruhstorfer: A. Seitz, Großschmett. Erde, v. 9, p. 420 (1911). - H. Stichel, Tierreich, Lief. 34, p. 154 (1912). 
subsp. domitilla (How.) Drusilla d., He- Batjan, witson, ut antea (part.), t. 8, f. 3, 4 (1861). Ternate - Tenaris d., Herrich-Schäffer, Corr.-BI. Zool.-Min. Ver. Regensb., v. 19, p. 89 (1865). - W. F. Kirby, Cat. Diurn. Lep., p. 118 (1871). - Drusilla d., E. Martens, Preuß. Exp. Ost-Asien, v. 1, p. 290 (1875). Tenaris d., O. Staudinger (\& Schatz), Exot. Schmett., v. 1, p. 192, 193 (part.) (1887). T. d. d., Fruhstorfer, Deutsch. Ent. 'Zeit. Iris, v. 15, 1902, p. 312 (1903). - Taenaris d. d., id., Wien. Ent. Zeit., v. 24, p. 120 (1905). - H. Stichel, Gen. Ins., fasc. 36, p. 42 (1906). - Fruhstorfer: A. Seitz, Großschmett. Erde, v. 9, p. 420 (1911). - H. Stichel, Tierreich, Lief. 34 , p. 155 (1912). Drusilla domitella (!), N. D. Riley \& Gabriel, Cat. Type Specim. Rhop. Brit. Mus., pars 1, p. 18 (1924).

subsp. diops (Snellen-Voll.) Drusilla d., Halmahera, Snellen v. Vollenhoven, Tijdschr. Ent., v. 6, p. Morotai 131, t. 8, f. 3" (1863). - Tenaris d., W. F. Kirby, Cat. Diurn. Lep., p. 118 (1871). T. damitilla diops., Fruhstorfer, Deutsch. Ent. Zeit. Tris, v. 15, 1902, p. 312 (1903). - Taenaris dom. diops, $T$. diana diops, Fruhstorfer, Wien. Ent. Zeit., v. 24, p. 120; p. $122(1905) .-T$. dom. diops, H. Stichel, Gen. Ins., fasc. 36, p. 42 (1906). - Fruhstorfer: A. Soitz, Großschmett. Erde, v. 9, p. 420 (1911). - H. Stichel, Tierreich, Lief. 34, p. 155 (1912).

Drusilla domitilla, Kirsch, Mitt. Zool. Mus. Dresden, v. 1. II, p. 121 (1877). Grose Smith, Nov. Zool., v. 1, p. 358 (1894). - subsp. a grippa (Fruhst.) Tenaris a., Fruhstorfer, Ins.-Börse, v. 20, p. 77 (1903).

T. domitilla a., id., Deutsch. Ent. Zeit. Iris, v. 15,1902 , p. 311 (1903). - Id., Berlin. Ent. Zoit., v. 49, Sitz.-Ber., p. (1) (1905). - Taenaris $d$. a., id., Wien. Ent. Zoit., v. 24, p. 120 (1905). - H. Stichel, Gen. Ins., fasc. 36, p. 42 (1906). - Fruhstorfer: A. Seitz, Großschmett. Erde, v. 9, p. 420 (1911). - T. d. a. forma principalis, H. Stichel, Tierreich, Lief. 34, p. 155, f. 22 (1912).

a grip $p$ in a Fruhst. T. d. a., Fruhstorfer: A. Seitz, Großschmett. Erde, v. 9, p. 420, t. $100 \mathrm{c}$; p. 1134 (corr.: agrippa) (1911 \& $27)$.

forma prodiga Fruhst. T. domitilla agrip$p a$ forma p., Fruhstorfer: A. Seitz, Großschmett. Erde, v. 9, p. 421 (1911). - H. Stichel, Tierreich, Lief. 34, p. 156 (1912).

onolaus (Kirsch) Drusilla o., Kirsch, Mitt. Zool. Mus. Dresden, V. 1. II, p. 122 (1877). Taenaris a., Fruhstorfer, Soc. Entom., v. 19, p. 139 (1904). - Id., Wien. Ent. Zeit., v. Obi 24, p. 125 (1905). - H. Stichel, Gen. Ins., 
fasc. 36, p. 43 (1906). - T. o. (part.), Fruhstorfer: A. Seitz, Großschmett. Erde, v. 1, p. 419 (1911). - H. Stichel, Tierreich, Lief. 34 , p. 156 (1912). - T. o., W. Rothschild, Nov. Zool., v. 23, p. 304 (1916). - Fruhstorfer, Ent. Rundschan, v. 34, p. 44 (1917).

subsp. o no la us (Kirsch) Drusilla o., Kirsch, ut antea, t. 6, f. 7 (1877). - Tenaris o., W. F. Kirby, Cat. Diurn. Lop., Suppl., App., p. 846 (1877). - Goss, Proc. Ent. Soc. London, p. XI (1888). - Brunner-Wattenwyl, Betracht. Farbenpr. Ins., p. 10, t. 6, f. 77 (1897). - T. o. o., Fruhstorfer, Soc. Entom. v. 19 , p. 139 (1904). - Taenaris o. o., id., Wien. Ent. Zeit., v. 24, p. 73, 120, 125 (1905). - H. Stichel, Gien. Ins., fasc. 36, p. 43 (1906). - Fruhstorfer: A. Seitz, Großschmett. Ende, v. 9, p. 418 (1911). - H. S.ichel, Tierreich, Lief. 34, p. 157 (1912).

subsp. macrophthalma Fruhst. T. honrathi macrophthalmus, Fruhstorfer, Wien. Ent. Zeit., v. 24, p. 73, 120 (1905). - Taenaris h. macrophthalma, H. Stichel, Gen. Ins., fasc. 36 , p. 42 (19ง6). - T. o. macrophthalmus, Frulustorfor: 1 . Seitz, Großschmett. Errde, v. 9, p. 420 (1911). - T. o. macrophthalma, H. Stichel, Tierreich, Lief. 34, p. 161 (1912).

subsp. ida (Honr.) Tenaris honrathi var. i., Hon rath, Berlin. Ent. Zeit., v. 33, p. 164 (1889). - Tenaris h. idae, Fruhstorfer, Soc. Entom., v. 19, p. 139 (1904). - Taenaris h. i., id., Wien. Ent. Zeit., v. 24, p. 73, 120 125 (1905). - T. o. ida, H. Stichel, Gen. Ins., fasc. 36, p. 43 (1906). - Fruhstorfer: A. Seitz, Großschmett. Erde, v. 9, p. 420 (1911). - H. Stichel, Tierreich, Lief. 34, p. 158 (1912). - W. Rothschild, Nov. Zool., v. 23, p. 304 (1916).

Tenaris onolaus ?, B. Hagen, Jahrb. Nassau. Ver.' Naturk., v. 50, p. 81 (1897).

subsp. enomia (Fruhst.) Tcnaris honrathi e., Fruhstorfer, Soc. Entom., v. 19, p. 129 (1904). - Taenaris h. e., id., Wien. Ent. Zeit., v. 24, p. 73, 120 (1905). - H. Stichel, Gen. Ins., fasc. 36, p. 42 (1906). - Fruhstorfer: A. Seitz, Großschmett. Erde, v. 9, p. 420 (1911). - H. Stichel, Tierreich, Lief. 34 , p. 161 (1912).

subsp. s a turatior (Fruhst.) Tenaris o. 8., Fruhstorfer, Ent. Zeit. Guben, v. 18, p. 118 (1904). - Id., Soc. Entom., v. 19, p. 139 (1905). - Taenaris o. 8., id., Wien. Ent. Zeit., v. 24, p. $73,120,125$ (1905). - H. Stichel, Gen. Ins., fasc. 36 , p. 43 (1906). - Fruhstorfer: A. Seitz, GroBschmett. Erde, v. 9, p. 420 , t. $100 \mathrm{~d}$ (1911). - H. Stichel, Tierreich, Lief. 34, p. 161 (1912). - W. Rothschild, Nov. Zool., v. 23, p. 304 (1916).

Inseln der

Geelvink Bai

(Mum, Passin

Rubi)

Jobi

Ostliches Süd-

Neu-Guinea

(Finsch -

hafen)

Südost-

Neu-Guinea

(Collingwood

Bai)

Westliches

Süd-

Neu-Guinea

(Port

MLoresby) 
Tenaris o nolaus (non Drusilla o. Kirsch), O. Staudinger (\& Schatz), Exot. Schmett., v. 1, t. 64 ; p. 192,196 (1886 \& 87).

subsp. mo $n \tan a$ Stich. T. o. m., H. Stichel, Westliches Gen. Ins., fase. 36, p. 43 (1906). - Fruh- Südstorfer: 4. Seitz, Großschmett. Erde, v. 9, p. 420 (1911). - II. Stichel, Tierreich, Lief. 34, p. 161 (1912). - W. Rothschild, Nov. Zool., v. 23 , p. 304 (1916).

Drusilla onolaus, Ch. Oberthür, Ann. Mus. Civ. Stor. Natural., v. 15, p. 509 (1880). - ? Tenaris molaus (!), Tryon: J. P. Thomson, Brit. N. Guinea, App., p. 258 (1892).

- Honrathi (Stauding.) Tenaris h., O. Staudinger (\& Schatz), Exot. Schmett., v. 1, t. 64 (1886). - Taenaris h., Fruhstorfer, Wien. Ent. Zeit., v. 24, p. 125,126 (1905). - H. Stichel, Gen. Ins., fasc. 36 , p. 42 (part.) (1906). W. Rothschild, Nov. Zool., v. 23, p. 304 (1916). - Fruhstorfer, Ent. Rundschau, v. 34, p. 44 (1917).

T. onola o $_{\text {s }}$ (part.), Fruhstorfer: A. Seitz, Großschmett. Erde, v. 9, p. 419 (1911). H. Stichel, Tierreich, Lief. 34, p. 156 (1912). subsp. Honrathi (Stauding.) Tenaris $h$., O. Staudinger, ut antea; p. 192, 195 (1886 \& 87). - Taenaris h. h., Fruhstorfer, Wien. Ent. Zeit., v. 24, p. 120 (1905). - H. Stichel, Gen. Ins., fasc. 36, p. 42 (1906). $-T$. onolaus $h$., Fruhstorfer: A. Seitz, GroBschmett. Erde, v. 9, p. 420 (1911). - T. $o . h$. forma principalis, H. Stichel, Tierreich, Lief. 34, p. 158 (1912). - T. h. h., W. Rathschild, Nov. Zool., v. 23, p. 304 (1916).

? Hyades (Tenaris) horsfieldi, Capronnier, Ann. Soc. Ent. Belgique, v. 30, p. 6 (1886).

forma $a b d$ on Frulist. $T$. onolaus honrathi Typ.: Waigeu forma a., Fruhstorfer: A. Seitz, Großschmet.t. Erde, v. 9, p. 420 (1911). - H. Stichel, Tierreich, Lief. 34, p. 159 (1912).

subsp. s e k arensis (Stauding.) Tenaris $h$. Nordost var. s., O. Staudinger (\& Schatz), Exot. Schmett., v. 1, p. 192, 195 (1887). - Taenaris h. s., Fruhstorfer, Wien. Ent. Zeit., v. 24, p. 71, 120, 125 (1905). - H. Stichel, Gen. Ins., fasc. 36, p. 42 (1906). - $T$. onolaus s.. Fruhstorfer: A. Seitz, Großschmett. Erde, v. 9, p. 420 (1911). - H. Stichel, Tierreich, Lief. 34, p. 159 (1912). - W. Rothschild (\& Durrant), Rep. Brit. Ornithol. Un. Exp. Dutcl-N. Guinea, 2, Nr. 15, p. 19 (1915). - Röber, Nova Guinea, v. 13, Zool., Livr. 1, p. 47 (1915). - T. honrathi s., W. Rothschild, Nov. Zool., v. 23, p. 304 Neu-Guinea (Sekar, Sorrong)

Waigeu,
Neu-Guinea

Waigeu

Neu-Guinea

Mount Epa,

? Hall-Sund,

Mount Obru (1916). 
subsp. Ritsemae (Fruhst.) T. $h$. $r$., Fruhstorfer, Soc. Entom., v. 19, p. 129, fig. (1904). - Taenaris $h . r_{.}, T$. r., id., Wien. Ent. Zeit., v. 24, p. $71,120,125$, t. 1 (1905). - H. Stichel, Gen. Ins., fasc. 36, p. 42 (1906). - $T$. onolaus $r$., Fruhstorfer: A. Seitz, Großschmett. Erde, v. 9, p. 420, t. 100 d; p. 1111 (1911 \& 27). - T. o. r. forma principalis, H. Stichel, Tierreich, Lief. 34 , p. 159 (cit. part.) (1912). - T. honrathi r., W. liothschild, Nov. Zool., v. 23, p. 3.44 (1916). $T$. onolaus $r$, Eecke, Nova Guinea, v. 15, Zool., p. 38 (1924).

Tenaris honrathi (non Staudinger), Groso Smith, Nov. Zool., v. 1, p. 358 (1894).

subsp. Rebeli (Fruhst.) Tenaris h. $r$.,

Fruhstorfer, Soc. Entom., v. 19, p. 129 (1904).

- Taenaris h. r., id., Wien. Ent. Zeit., v. 24,

p. $72,120,125$ (1905). - T. h. ritsemae forma $r_{\text {., }} \mathrm{H}$. Stichel, Gen. Ins., fasc. 36, p. 42 (1906). - $T$. onolaus $r$., Fruhstorfer: A. Seitz, G roßschmett. Erde, v. 9, p. 420 (1911). - T. o. ritsemae forma rebeli, $\mathrm{H}$. Stichel, Tierreich, Lief. 34, p. 160 (1912). - T. honrathi rebeli, W. Rothschild, Nov. Zool., v. 23 , p. 304 (1916).

Tenaris $h$. var. selarensis (non Staudinger), B. Hagen, Jahrb. Nassau. Ver. Naturk., v. 50, p. 81 (variatio) (1897). - T. h. s., Rebel, Termesz. Füzet., v. 21, p. 374, t. 18 , f. 13 (1898).

forma a u $r$ ifl $l$ a Fruhst. .T. $h$. r. ab. $a$ Fruhstorfer, Wien. Ent. Zeit., v. 24, p. 72, 120 (1905). - T. h. r. forma a., H. Stichel, Gen. Ins., fasc. 36, p. 42 (1906). $-T$. onotaus (a.), Fruhstorfer: A. Seitz, Großschmett. Erde, v. 9, p. 419 (1911). - T. o. ritsemae forma a., $\dot{H}$. Stichel, Tierreich, Lief. 34, p. 160 (1912).

forma $s$ id $u s$ Stich. T. $h$. ritsemae forma s., Typ.:

H. Stichel, Gen. Ins., fasc. 36, p. 42 (1906). Astrolabe

- T. onolaus. (s.), Fruhstorfer: A. Seitz, Bai Großschmett. Erde, v. 9, p. 419 (1911). T. o. ritsemae forma $8 .$, H. Stichel, Tierreich, Lief. 34 , p. 160, f. 23 (1912).

\section{Cohors d. Selenidiformes}

H. Stichel, Gen. Ins, fasc. 36, p. 43 (1906). - Id., Tierreich, Lief. 34, p. 162 (1912).

- phorcas (Westw.) Drusilla p., Westwood, Trans. BismarckEnt. Soc. London, ser. 2, v. 4, p. 182 (1858). Archipel, - Taenaris p., Fiuhstorfer, Wien. Ent. Zeit., Salomonv. 24 , p. 126 (1905). - H. Stichel, Gen. Inseln, Ins., fasc. 36, p. 43 (1906). - Fruhstorfer: NeuA. Seitz, Großschmett. Erde, v. 9, p. 419 Hebriden (1911). - H. Stichel, Tierreich, Lief. 34, p. 162 (1912).

Lepidopterorum Catalogus 54. 
Te naris a nableps, $\Lambda$. Pagenstecher, Zoologica, Heft 27, p. 92 (1899).

subsp. phorcas (Westw.) Drusilla p., West- Neuwood, ut antea, t. 21, f. 1. (1858). - Tenaris p., Herrich-Schäffer, Corr.-Bl. Zool.-Min. Ver. Regensb., v. 19, p. 89 (1865). - W. F. Kirby, Cat. Diurn. Lep., p. 118 (1871). O. Staudinger (\& Schatz), Exot. Schmett., v. 1, p. 193, 199 (1887). - Taenaris p. p., Frubstorfer, Wien. Ent. Zeit., v. 24, p. 120 (1905). - H. Stichel, Gen. Ins., fasc. 36, p. 44 (part.) (1906). - Fruhstorfer: A. Seitz, Großschmett. Erde, v. 9, p. 419, t. 100d (umbonia, corr. p. 1134) (1911 \& 27). T. $p . p$. forma principalis, H. Stichel, Tierreich, Lief. 34, p. 163 (1912). - Drusilla p., N. D. Riley \& Gabriel, Oat. Type Spec. Rhop. Brit. Mus., pars 1, p. 46 (1924).

a nabiep 8 (Snell. v. Voll.) Drusilla a., Snellen v. Vollenhoven, Tijdschr. Entom., v. 3, t. 1, f. 3 ; t. 2 , f. 1 (1860). - Tenaris a., Herrich-Schäffer, Corr:-Bl. Zool-Min. Ver. Regensb., v. 19, p. 89 (1865). - W. F. Kirby, Cat. Diurn. Lop., p. 118 (1871). - ? A. G. Butler, Proc. Zool. Soc., p. 280 (? patria falsa) (1874). - Drusilla a., F. D. Godman \& O. Salvin, Proc. Zool. Soc. London, p. 157 (1879). - Tenaris phorcas var. a., O. Staudinger (\& Schatz), Exot. Schmett., v. 1, p. 193, 199 (1887). - T. a., Ribbe, Deutsch. Ent. Zeit. Lep., v. 9, p. 108 (1898). $-T$. phorcas a., Fruhstorfer, Wien. Ent. Zeit., v. 24, p. 120 (1905).

? Drusilla catops (non Westwood) + $D$. selene (non Westwood), Hewitson, Proc. Zool. Soc. London, p. 52 (1861). - ? D. 8. + D. c., Kirsch, Mitt. Zool. MLus. Dresden, v. 1 , p. $120(1877)$.

subsp. $u m b$ on ia Fruhst. T. phorcas umbonius, T. p. umbonia, Fruhstorfer, Wien. Ent. Zeit., v. 24, p. 87, 120 (1905). - T. p. p. forma umbonia, H. Stichel, Gen. Ins., fasc. 36, p. 44 (1906). - T. p. u., Fruhstorfer: A. Seitz, Großschmett. Ende, v. 9, p. 419 (non t. 100d: $=$ phorcas p., conf. p. 1134) (1911 \& 27). T. p. p. forma u., H. Stichel, Tierreich, Lief. 34 , p. 163 (1912).

subsp. $и$ ran $и s$ (Stauding.) Tenaris $p$. var. (P) u., O. Staudinger (\& Schatz), Exot. Schmett., v. 1, p. 193, 200 (1887). - IT. u., E. Hofmann, Jahrosh. Ver. Naturk. Württemb., v. 46, p. 236 (Organ. odor.) (1890). - T. phorcas var. u., Ribbe, Doutsch. Ent. Zeit. Lep., v. 9, p. 109 (1898). - Taenaris p. u., Fruhstorfer, Wien. Ent. Zeit., v. 24 , p. 120 (1905). - H. Stichel, Gen. Ins., fasc. 36 , p. 44, t. 5 , f. 14 (q) (1906). Fruhstorfer: A. Seitz, Großschmett. Erde, Y. 9, p. 419 (1911). - H. Stichel, Tier-

\author{
Mecklenburg \\ $(=$ Neu- \\ Irland)
}

Neu-

Hannover

(ExpeditionsBai)

Neu-

Pommern

(=Nou-

Britannien),

? Nou-

Lauenburg

(Duke-of-

York-Insel) 
reich, Lief. 34, p. 164 (1912). - Strand, Int. Ent. Zeit., v. 8, p. 103 (1914); id., Lepid. Niepelt., v. 2, p. 11 (1916).

? T. an ableps, T. a. ab. O (non Drusilla a. Snell: v. Voll.), O. Salvin \& F. D. Godman, Proc. Zool. Soc. London, p. 143 (? patria falsa) (1877). - T. a., W. F. Kirby, Cat. Diurn. Lep. Suppl. App., p. 846 (1877). A. Pagenstecher, Jahrb. Nassau. Ver. Naturk., v. 47, p. 76 (1894). - B. Hagen, l. c., v. 50 , p. $82(1897)$.

subsp. admiralitatis Rothsch. T. p. a., AdmiralitätsW. Rothschild, Nov. Zool., v. 23, p. 304 (1916).

T. p. uranus (non Stauding.), IV. Rothschild, Nov. Zool., v. 22, p. 201 (1915).

subsp. at esta (Rebel) Tenaris a., Rebel, Salomon-

Verh. Zool.-Bot. Ges. Wien, v. 45, p. 106 Inseln (1895). - Ribbe, Deutsch. Ent. Zeit. Lep., v. 11, p. 109 (1898). - Taenxris phorcas a., Fruhstorfer, Wien. Ent. Zeit., v. 24, p. 120 (1905). - II. Stichel, Gen. Ins., fusc. 36, p. 44 (1906). - ? Tenaris uranus a., Hauser, Verh. Zool.-Bot. Ges. Wien, v. 60, p. (30) (? determin. errans) (1910). - Taenaris phorcas a., Fruhstorfer: A. Seitz, Großschmett., v. 9, p. 419 (1911). - T. p. a. forma principalis, H. Stichel, Tierroich, Lief. 34, p. 165 , f. 24 (1912). - T. a., Strand, Int. Ent. Zeit., v. 8, p. 103 (1914); id.', Lepid. Niepelt., v. 2, p. 11 (1916).

Tenaris phorcas (non Drusilla p. Westwood), A. G. Butler, Proc. Zool. Soc. London, p. 279 (1874).

$T$. uranus (non T. phorcas var. $u$. Stauding.), Ribbe, Deutsche Ent. Zeit. Lep., v. 8 , p. 113, t. 3, f. 10,11 (larva, chrysal.). (1895).

forma bougainvilleana Strand $T$. $p$. Typ.:

ab. b., Strand, Int. Ent. Zeit., v. 8, p. 104

(O) (1914). - Id., Lep. Niepeltian., pars 2 , p. 11, t. 16, f. 14 (1916). - Id., Bull. So: Zool. France, v. 51, p. 397 (1926) (recte: 1927).

subsp. far o n a Fruhst. T. p. f., Fruhstorfer: Faro

A. Seitz, Großschmett. Erde, v. 9, p. 419 (= Fauro), (1912) (an forma praeced.?)

scylla (Stauding!) Tenaris s., O. Staudingor Shouten(\& Schatz), Exot. Schmett., v. 1, p. 193, Insel 200 (1887). - Taenaris s, Fruhstarfer, (Misore, Wien. Ent. Zeit., v. 24, p. 120, 126 (1905). Geelvink - H. Stichel, Gen. Ins., fasc. 36, p. 44 (1906). - Fruhstorfer: A. Seitz, Großschmett. Erde, v. 9, p. 419 (1911). - H. Stichel, Tierreich, Lief. 34, p. 165, f. 25 (1912). - W. Rothschild, Nov. Zool., v.

Inseln

(St. Gabriel, Manus)

Bougainville

$\mathrm{Bai})$ 23, p. 304 (1916). 
Dohertyi (Gr. Smith) Tenaris d., Grose Smith, Nov. Zool., v. 1, p. 358 (1894). Grose Smith \& W. F. Kirby, Rhop. Exot., v. 2, t. Tenaris 3, f. 3, 4, p. 11 (1895).

Drusilla phorcas (non Westwood), Kirsch, Mitl. Zoul. Mlus. Dresde:1, v. 1. 11, p. 121 (18\%7). macrops (Feld.) Drusilla m., O. \& R. Felder, Wien. Ent. Monatschr., v. 4, p. 218 (1860). Taenaris m., H. Stichel, Gen. Ins., fasc. 36, p. 44 (1906). - Fruhstorfer: A. Seitz, Großschmett. Erde, v. 9, p. 416 (1911). - H. Stichel, Tierreich, Lief. 34, p. 167 (1912).

$T$. sele ne (part.), Fruhstorfer, Wien. Ent. Zeit., v. 24, p. 126 (1905).

subsp. macrops (Feld.) Drusilla m., C. \& Halmahera, T. Felder, ut antea (1860). - Tenaris $m$. Herrich-Schäffer, Corr.-Bl. Zool.-Min. Ver. Regensb., v. 19 , p. 89 (1865). - Hyades $m$. C. \& R. Felder, Reise Novara, v. 2. II, p. $46 \mathrm{~J}$ (1866). - Tenaris m., W. F. Kirby, Cat. Diurn. Lep., p. 118 (1871). - Kirsch, Mitt. Zool. Mus. Divesden, v. 1, p. 120 (T. selene var.) (1877). - T. m., Ch. Oberthür, Ann. Mus. Civ. Stor. Natural., v. 15, p. 510 (? selene modif.) (1880). - T. $m, 0$. Staudinger (\& Schatz), Ixxot. Schmett., v. 1, p. 192, 194 (part.) (1887). - E. Lucuter, Acta Soc. Sci. Fenn., v. 22, p. 110 (1896). - A. Pagenstecher, Abh. Senckenb. Naturf. Ges., v. 23, p. 405 (purt.) (1897). - T. m., T. selene $m$., Frubstorfer, Wien. Ent. Zeit., v. 24, p. 86, 121 (1905). - T. m., H. Stichel, Gen. Ius., fasc. 36, p. 41 (part.) (1906). T. m. m., Fruhstorfer: A. Seitz, GroßGroßsclimett. Erde, v. 9, p. 417 (1911). $T$. $m$. forma principalis, H. Stichel, 'Tierreich, Lief. 34, p. 167 , f. 26 (Q) (1912).

Drusilla artemis (?) + D. anableps (?) + D. dioptrica (?), Hewitson, Proc. Zool. Soc. London, p. 52 (1861).

Drusilla selene $+D$. catops, Kirsch, Mitt. Zool. Mus. Dresden, v. 1. II, p. 120 (conf. etiam phorcas) (1877).

subsp. macropina (Fruhst.) Tenaris ma- Obi crops macropina, Fruhstorfer, Soc. Entom., v. 19, p. 26 (1904). - Taenaris macropina, $T$. selene $m$., Fruhstorfer, Wien. Ent. Zeit., v. 24, p. 86,121 (1905). - T. macrops forma macropina, H. Stichel, Gen. Ins., fasc, 36 , p. 44 (1906). - 'T. macrops macropina, Fruhstorfer: A. Seitz, Großschmett. Erde, v. 9, p. 417, t. 101 a (1911). - T. macrops forma macropina, H. Stichel, Tierreich, Lief. 34, p. 168 (1912).

Tenaris macrop8, O. Staudinger (\& Schatz), Exot. Schmett., v. 1, p. 192, 194 (part.) (1887). - A. Pagenstecher, Abh. Senckenb. Naturf. Ges., v. 23, p. 415 (part.) (1897).

Batjan,

Morotai 
subsp. ternatana Fruhst. Taenaris ma-Ternate crops t., Fruhstorfer, Ent. Zeit. Stettin, v. 70, p. 226 (1909). - Id.: A. Seitz, Großschmett. Erde, v. 9, p. 417 (1911).

$T$. $m$. forma $t ., \mathrm{H}$. Stichel, Tierieich, Lief. 34, p. 168 (1912).

Tenaris macrops, Ch. Oberthür, Ann. Nus. Civ. Stor. Natural., v. 15, p. 510 (part.) (1880). - A. Pagenstecher, Abh. Senckenb. Naturf. Ges., v. 23, p. 415 (part.) (1897).

selene (Westw.) Drusilla s. (E. Doubleday \&) Westwood, Gen. Diurn. T,ep., v. 2, p. 335 (1851). - Taenaris s., Fruhstorfer, Wien. Ent. Zeit., v. 24, p. 126 (part.) (1905). - H. Stichel, Gen. Ins., fasc. 36, p. 44 (1906). - Fruhstorfer: A. Seitz, Großschmett. Erde, v. 9, p. 416 (1911). - H. Stichel, Tierreich, Lief. 34, p. 168 (1912).

subsp. selene (Westw.) Drusilla 8. (Hyades Buru 8. Boisduval MS), Westwood, ut antea (patria falsa) (1851). - D. s., Chenu \& H. Lucas, Enc. Hist. Nat. Papill., p. 166 (1851). Westwood, Trans. Ent. Soc. London, n. ser., v. 4, p. 181 (1858). - Id., Proc. Zool. Soc. London, p. 52 (1861). - Tenaris s., Herrich-Schälfer, Corr.-Bl. Zool.-Min. Reg., v. 19 , p. 89 (1865). - W. F. Kirby, Cat. Diurn. Iep., p. 118 (1871). - Id., Śi. Proc. R. Dublin Soc., ser. 2, v. 2, p. 300 (1880).

Taenaris s. s., Fruhstorfer, Wien. Ent. Zeit., v. 24, p. 121 (1905). - T. s., H. Stichel, Gen. Ins., fasc. 36 , p. $45^{\circ}$ (cit. part.), t. 6, f. 15 (Q) (1906). - T. s. 8., Fruhstorfor: A. Seitz, Großschmett. Erde, v. 9, p. 416, t. 101 a (1911). - T. s. forma principalis, $H$. Stichel, Tierreich, Lief. 34, p, 168, 169 (cit part.) (1912). - T. s., Eecke, Treubia, v. 7, p. 359 (1929).

buruensis (Forbes) Tenaris b., Forbes, Natural. Wander. East-Archipel, p. 411 (1883). - C. O. Waterhouse, Aid Identif. Ins., p. 15, t. 166 (Q) (patria falsa) (1886). - Iolland, Nov. Zool., v. 7, p. 62 (1900). ? Drusilla selene var. catops, Kirsch, Mitt. Zool. Mus. Dresden, v. 1, p. 120 (conf. phorcas) (1877).

subsp. gigas (Stauding.) Tenaris macrops var. g., O. Staudinger (\& Selutz), Exot. Schmett., v. 1, p. 192, $194\left(\sigma^{7}\right)$ (1887). Ribbe, Deutsch. Ent. Zeit. Lep., v. 2, p. 241 (1889). - Röber, Tijdschr. Ent., v. 34, p. 309 (1891). - T. g., Fruhstorfer, Wien. Ent. Zeit., v. 24, p. 121 (1905). - T. selene g., Fruhstorfer: A. Seitz, Großschmett. Erde, v. 9, p. 416 (1911). - W. Rothschild, Nov, Zool., v. 22, p. 124 (1915).

$T$. s e l e $n$ e (part.), H. Stichel, Gen. Ins., fasc. 36, p. 44 (1906). - Id., Tierreich, Lief. 34, p. 168 (1912).

Buru,

Ceram 
forma tetrica Stich. T. selene forma $t$., H. Typ.: Ceram Stichel, Gen. Ins., fasc. 36, p. 45 (1906). T. s. gigas "Form" t., Fruhstorfer: A. Seitz, Großschmett. Erde, v. 9, p. 416 (1911). $T$. s. forma $t$., H. Stichel, Tierreich, Lief. 34 , p. 170 (1912).

forma pseudomacrops Rothsch. T. s. Typ.: forma m., W. Rothschild, Nov. Zool., v. 22, Zentralp. 125 (1915).

Ceram

- catops (Westw.) Drusilla c., (E. Doubleday \&) Westwood, Gen. Diurn. Lep., v. 2, p. 335 (1851). - Taenaris c., Fruhstorfer, Wien. Ent. Zeit., v. 24, p. 125, 126 (1905). - H. Stichel, Gen. Ins., fase. 36, p. 45 (1906). Fruhstorfer: A. Seitz, Großschmett. Erde, v. 9, p. 417 (1911). - Strand, Mitt. Zool. Mus. Berlin, v. 5, p. 470 (1911). - H. Stichel, Tierreich, Lief. 34 , p. 170 (1912).Eecke, Nova Guinea, v. 13, Zool. Livr. 1, p. 68 (1915).

subsp. catop 8 (Westw.) Drusilla c., (IIyades c. Boisduval MS), Westwood, ut antea, p. 335 (patria falsa); p. 533 (1851 \& 52). Chenu \& H. Lucas, Enc. Hist. Nat., Papill., p. 166 (1851). - Westwood, Trans. Ent. Soc. London, n. ser., v. 4, p. 181 (1858). Tenaris c., Herrich-Schäffer; Corr.-Bl. Zool.Miu. Ver. Regensb., v. 19, p. 89 (1865). W. F. Kirby, Cat. Diurn. Lep., p. 118 (1871). - A. G. Butler, Proc. Zool. Soc. London, p. 279 (distr. part.) (1874). - Drusilla c., Ch. Oberthür, Ann. Mus. Civ. Stor. Natural. Genova, v. 15, p. 509 (part.) (1880). Tenaris c., A. G. Butler, Ann. Mag. Nat. Hist., ser. 5, v. 11, p. 402, 411 (1883). Ribbe, Carr.-Bl. Ent. Ver. Iris, v. 1, p. 82 (1886). - O. Staudinger (\& Schatz), Exot. Schmett., v. 1, p. 192, 197 (part.) (1887). - T. c. c., Truhstorfer, Wien. Ent. Zeit., , 24 , p. 84,121 (1905). - H. Stichel, Gen. Ins., fasc. 36, p. 45 (1906). - Truhstorfer: A. Seitz, Großschmett. Erde, v. 9, p. 417 (1911). - T. c., A. Pagenstecher, $\mathrm{Abh}$. Senckenb. Naturf. Ges., v. 33, p. 411 (1911). - T. c. c., H. Stichel, Tierreich, Lief. 34, p. 171 , f. 27 (1912).

Drusilla selene (non Westwood), Kirsch, Mitt. Zool. Mus. Dresden, v. 1, p. 120 (1877). - Ch. Oberthür, Ann. Mus. Civ. Stor. Natural. Genova, v. 15, p. 509 (1880). - T. catops var. 8., 0 . Staudinger (\& Schatz), Exot. Schmett., v. 1, p. 509 (part.) (1887). Tenaris pamphagus (non Drusilla p. Kirsch), Ribbe, Corr.-Bl. Ent. Ver. Iris, v. 1, p. 82 (1886). - ? A. Pagenstecher, Abh. Senckenb. Naturf. Ges., v. 33, p. 411 (1911). subsp. fulvida (Butl.) Tenaris f., A. G. Butler, Trans. Ent. Soc. London, p. 487

Neu-Guinea und benachbarte Inseln

Aru-Inseln 
(1870). - W. F. Kirby, Cat. Diurn. Lep., App., p. 646 (1871). - O. Staudinger (\& Schatz), Exot. Schmett., v. 1, p. 193, 198 (1887). - Taenaris catops f., Fruhstorfer, Wien. Ent. Zeit., v. 24, p. 85, 198 (1905). -

H. Stichel, Gen. Ins., fasc. 36, p. 46 (1906).

- Fruhstorfer: A. Seitz, Großschmett. Erde, v. 9, p. 417 (1911). - H. Stichel, Tierreich, Lief. 34, p. 178 (1912). - W. Rothschild, Nov. Zool., v. 22, p. 125 (1915). Tenaris $f$., N. D. Riley \& Gabriel, Cat. Type spec. Rhop. Brit. Mus., pars 1, p. 21 (1924).

subsp. $j a t h r i p p a$ Fruhst. T. c. j., Fruhstorfer, Arch. Naturg., v. 81, 1915, Abt. A, Heft 11, p. 65 (1916).

subsp. selenides (Stauding.) Tenaris c. Waigeu var. 8., O. Staudinger (\& Schatz), Exot. Schmett., v. 1, p. 192, 198 (1887). - Tae. naris c. s., Fruhstorfer, Wien. Ent. Zeit., จ. 24, p. 86,121 (1905). - H. Stichel, Gen. Ins., fasc. 36 , p. 46 (1906). - Fruhstorfer: A. Seitz, Großschmett. Erde, v. 9, p. 417, t. 101 a (1911). - $T$. c. s. forma principalis, H. Stichel, Tierreich, Lief. 34, p. 177, 178 (distr. part.) (1912).

forma $d$ e $l u$ n a $t$ a Fruhst. $T$. c. s. forma $d$. ., Typ.:

Fruhstorfer: A. Seitz, Großschmett. Erde, Waigeu v. 9, p. 417 (1911). - H. Stichel, Tierreich, Lief. 34 , p. 178 (1912).

forma attina Fruhst. T. c. s., forma a., Typ.:

Fruhstorfer: A. Soitz, Großschmett. Erde, Waigeu

v. 9 , p. 417 , t. 101 a (1911). - H. Stichel,

Tierreich, Lief. 34, p. 178 (1912).

subsp. laretta (Fruhst.) Tenaris c. l., Nördl.

Fruhstorfer, Soc. Ent., v. 19, p .129 (1904).

- Taenaris c. l., Fruhstorfer, Wien. Ent.

Zeit., v. 24, p. 85, 121, 125, t. 2 (1905). -

Id.: A. Seitz, Großschmett. Erde, v. 9, p.

417 (1911). - Röber, Nov. Guinea, v. 13,

Zool., livr. 1, p. 47 (distr. part.) (1915). -

Eecke, Nova Guinea, v. 15, Zool., p. 37 (1924).

T. c. pamphaga (non Drusilla p. Kirsch) (part.), H. Stichel, Gen. Ins., fasc. 36, p. 46 (1906). - Id., Tierreich, Iief. 34, p. 176, f. 30 (1912).

subsp. jobina (Fruhst.) Tenaris c. j., GeelvinkFruhstorfer, Soc. Ent., v. 19, p. 129 (1904). - Taenaris c. j., id., Wien. Ent, Zeit., v. 24, p. 85 (1905). - H. Stichel, Gen. Ins. fasc. 36, p. 46 (1906). - Fruhstorfer: A. Seitz, Großschmett. Erde, v. 9, p. 418 (1911). - H. Stichel, Tierreich, Lief. 34, p. 176 (1912). - T. c. "rnce" j., Eecke, Nova Guinea, v. 13, Zool., livr. 1, p. 68 (1915).

- subsp. pamphaga (Kirsch) Drusilla pamphagus, Kirsch, Mitt. Zool. Mus. Dresden, v. 1, p. 120 (? distr. part.) (1877). - Taenaris catops pamphagus, Fruhstorfer, Wien.

HolländischNou-Guinea (Sorrong)
HolländischNeu-Guinea (Dorey, Passim) 
Ent. Zeit., v. 24, p. 86, 121, 125 (1905). Taenaris c. pamphaga, H. Stichel, Gen. Ins., fasc. 36 , p. 46 (1906). - T. c. pamphagus, Fruhstorfer: A. Seitz: Großschmett. Erde, v. 9, p. 418 (1911). - ? D. c. pamphagus, Strand, Mitt. Zool. Mus. Berlin, v. 5, p. 470 (? determ. errans) (1911). - D. c. pamphaga, H. Stichel, Tierreich, Lief. 34, p. 176 (non f. $30:=T$. c. laretta) (1912). ? Fecke, Nova Guinea, v. 15, Zool., p. 37 (1924).

? Tenaris catops, Kirsch, Mitt. Zool. Mus. Dresden, v. 1, p. 120 (1877). - ? Grose Smith, Nov. Zool., v. 1, p. 359 (1894).

Drusilla selene (non Westwood), Ch. Oberthür, Ann. Mus. Civ. Stor. Natural., Genova, v. 12, p. 462 (1878). - Id., 1. c., v. 15 , p. 509 (1880). - Tenaris catops var. (et ab.) 8., O. Staudinger (\& Schatz), Exot. Schmett., v. 1, p. 192, 197 (1887).

T. c. laretta + T. c. catanea (part. in cit.), H. Stichel, Gen. Ins., fasc. 36 , p. 46 (1906). - Id., Tierreich, Lief. 34, p. 175 (1912).

subsp. colarima Eecke $r$. catops colarima, HolländischEecke, Nova Guinea, v. 13, Zool. 1, p. 68, Neu-Guinea t. 3 , f. 8,8 a $\left(\sigma^{n}, 9\right)(1915)$.

subsp. dig u li ca Hulst. T. c. d., Hulstaert, Ann. Mag. Nat. Hist., ser. 9, v. 15, p. 445 (1925).

(nördlich, zentral)

Süd-

HolländischNeu-Guinea (Digul)

forma $H u l$ staerti (nov. nom. pro vanhaasterti, praeocc.: $C$. rothschildi v., Hulstaert). T. c. Vanha a sterti, Hulstaert, Ann. Mag. Nat. Hist., v. 19, p. 446 (1925).

subsp. $t u r a$ ica Hulst. $r$. c. $t_{\text {. }}$, Hulstaert, SüdAnn. Mag. Nat. Hist., ser. 9, v. 15, p. 446 (1925).

Typ.: Digul

subsp. kajuna (Fruhst.) Tenaris c. k., Fruhstorfer, Soc. Entom., v. 19, p. 119 (1904). - Taenaris c. k., id., Wien. Ent. Zeit., v. 24, p. 84, 121, 125 (1905). - H. Stichel, Gen. Ins., fase. 36, p. 46 (1906). - Fruhstorfer: A. Seitz, Großschmett. Erde, v. 9, p. 418 (1911). - H. Stichel, Tierreich, Lief. 34, p. 175 (1912).

subsp. catanea (Fruhst.) Tenaris catops catanea, Fruhstorfer, Soc. Entom., v. 19, p. 129 (1904). - Taenaris catops catanea, id. Wien. Ent. Zeit., v. 24, p. 84, 121, 125 (1905). - Id.: A. Seitz, Großschmett. Erde, v. 9, p. 418 (1911). - T. catops "races" catanea, Eecke, Nova Guinea, v. 13, Zool., livr. 1 , p. 68 (1915). - T. catops catanea, W. Rothschild (\& Durrant), Rep. Brit. Ornithol. Un. Exped. Dutch N. Guinea, 2, Nr.

HolländischNeu-Guinea (Turai)

Holländisch-

Neu-Guinea (Kajumera Bai)

HolländischNou-Guinea (Kapaur, Arguni Bai) 
15, p. 19 (1915). - Fruhstorfer, Arch. Naturg., v. 81,1915 , Abt. $A$, Heft 11, p. 65 (1916).

T. c. pa mphaga (non Drusilla pamphagus Kirsch) (part. in cit.), H. Stichel, Gen. Ins., fasc. 36 , p. 46 (1906). - Id., Tierreich, Lief. 34, p. 175 (1912).

subsp. Vaneeckei Hulst. T. c. v., Hulstaert, Ann. Mag. Nat. Hist., ser. 9, v. 11, p. 184 (1923).

subsp. gal a c c ia Fruhst. T. c. g., Fruhstorfer, Ent. Rundschau, v. 31, p. 26 (1914).

- subsp. We stwoodi (Stauding.) Tenaris c. var. $w$. , O. Staudinger, Deutsch. Ent. Zeit. Iep., v. 6, p. 368 (1893). - T. w., Fürbringer (\& A. Pagenstecher): Semon, Forschungsreis. 5, Denkschr. Med.-Naturw. Ges. Jena, v. 8, p. (78) 238 (1895). - Taenaris c. w., T. w., Fruhstorfer, Wion. Ent. Zeit., v. 24 , p. 121, 124, 125, t. 1 (larva) (1905). T. c. $w ., \mathrm{H}$. Stichel, Gen. Ins, fasc. 36, p. 45 (1906). - Tenaris w., Hauser, Verh. Zool.-Bot. Ges. Wien, v. 60, p. (38) (1910). - Taenaris c. w., Fruhstorfer: A. Seitz, Großschmett. Erde, v. 9, p. 418 (1911). - Strand, Archiv f. Naturg. 1912, A. 11, p. 65 . - Strand, Mitt. Zool. Mus. Berlin, v. 5, p. 470 (1911) T. c. $w$. forma principalis, H. Stichel, Tierreich, Lief. 34 , p. 173, f. 28 (Q) (1912).

Drusilla catops (non Westwood), F. D. Godman \& O. Salvin, Proc. Zool. Soc. Lond., p. 645 (part.) (1878). - Tenaris c., O.Staudinger (\& Schatz), Exot. Schmett., v. 1, p. 192, 197 (1887). - B. Hagen, Jahrb. Nassau. Ver. Naturk., v. 50, p. 79 (part.) (1897): - Rebel, Termesz. Füzet., v. 21, p. 324 (1898).

Tenaris c. var. (et ab.) selene (non Drusilla 8. Westwood), O. Staudinger (\& Schatz), Exot. Schmctt., v. 1, p. 192, 197 (part.) (1887). - ? B. Hagen, Jahrb. Nassau. Ver. Naturk, v. 50, p. 80 (1897).

forma unipupill a t a Fruhst. T. c. w. ab. u., Fruhstorfer, Soc. Entom., v. 20, p. 35 (1905). - Taenaris c. w. ab. u., Id., Wien. Ent. Zeit., v. 24, p. 121 (1905). - T. c. $w$. forma $u$., $\mathrm{H}$. Stichel, Gen. Ins, fasc. 36, p. 45 (1906). - T. c. u., Fruhstor,fer: A. Seitz, Großschmett. Erde, v. 9, p. 418 (1911). - T. c. $w$. forma $u$., H. Stichel, Tierreich, Lief. 34 , p. 174 (1912).

forma inter rupta Strand T. c. i., Mitt. Typ.:

Zool. Mus. Berlin, v. 5, p. 470 (1911). - Id., Ehemal.

Bull. Zool. France, v. 51, p. 402 (1927). Dieutsch-

Neu-Guinea

Holländisch

Neu-Guinea

(Merauke-

Fluß)

Holländisch-

Neu-Guinea

(Eilanden-

Fluß)

Mittleres

Nordost-

Neu-Guinea

(Finschküste.

Astrolabe

Bai)

Typ.:

Ehemal.

Neu-Guinea 
subsp. l un a Stich. T. c. l., H. Stichel, Gen. NordostIns., fasc. 36 , p. 46 (1905). - T. c. "Form" l., Fruhstorfer: A. Seitz, Großschmett. Erde, v. 9, p. 418 (1911). - T. c. l. (?), Strand, Mitt. Zool. Mus. Berlin, v. 5, p. 470 (1911). - T. c. l., H. Stichel, Tierreich, Lief. 34, p. 177 (1912).

subsp. vulcania (Fruhstorfer MS), T. c. Nordostwestwoodi forma v., H. Stichel, Tierreich, Lief. 34 , p. 174 (1912). - T. c. vulcanica, W. Rothschild, Nov. Zool., v. 22, p. 201 (1915). T. c. myalechoides (!), Fruhstorfer, Neu-Guinea (Seleo, Berlinhafen)

Deutsch. Ent. Zeit. Iris, v. 24, p. 56 (1910). - T. c. mylaechoides (part.), id., A. Seitz, Großschmett. Erde, v. 9, p. 418 (1911).

forma $s u b$ mylaecha Rothsch. T. c. vulcanica ab. s., W. Rothschild, Nov. Zool,, v. 22, p. 201 (1915).

subsp. dampierensis Rothsch. T. c. d., W. Rothschild, Nov. Zool., v. 22, p. 201 (1915).

T. catops, B. Hagen, Jahrb. Nassau. Ver. Naturk., v. 50, p. 79 (part.) (1897).

forma $s u b l u n a$ Rothsch. T. c. dampierensis ab. s., W. Rothschild, Nov. Zool., v. 22, p. 202 (1915).

subsp. mylaechoides Frubst. T. c. m., Fruhstorfer, Wien. Ent. Zeit., v. 24, p. 83 (1905). - T. c. westwoodi forma m., II. Stichel, Gen. Ins., fasc. 36, p. 45 (1906). T. c. m., Fruhstorfer: A. Seitz, Großschmett. Erde, v. 9, p. 418 (1911). - T. c. westwoodi forma m., II. Stichel, Tierreich, Lief. 34 , p. 174, f. 29 (q) (1912). - T. c. ,race" jobina O-forma m., Eecke, Nova Guinea, v. 13, Zool., livr. 1, p. 68 (1915).

? Tenaris catops (part.), F. D. Godman \& O. Salvin, Proc. Zool. Soc. London, p. 645 (1878).

subsp. adriana (Fruhst.) Tenaris c. a., Fruhstorfer, Soc. Ent., v. 19, p. 129 (1904). - Taenaris c. a., id., Wien. Ent. Zeit., v. 24, p. 83, 121 (1905). - Id.: A. Seitz, Großschmett. Erde, v. 9, p. 418 (1911).

Drusilla catops (part.), F. D. Godman \& O. Salvin, Proc. Zool. Soc. London, p. 645 (1878).

T. c. fimbriata (non T. f. Kirby) (part.), H. Stichel, Gen. Ins., fasc. 36 , p. 45 (1906). - Id., Tierroich, Lief. 34, p. 174 (1912).

subsp. fimbriat a (Kirby) Tenaris $f .$, W. LouisiadeF. Kirby, Ann. Mag. Nat. Hist., ser. 6, v. 3 , p. 160 (1889). - Taenaris catops $t .$. Fruhstorfer, Wien. Ent. Zeit., v. 24, p. 121 (1905). - H. Stichel, Gen. Ins., fasc. 36, p. 45 (1906). - Fruhstorfer: A. Seitz, Großschmett. Erde, v. 9, p. 418 (1911).- H. Stichel, Tierreich, Lief. 34, p. 174 (part.) Neu-Guinea (Vulkaninsel - Manam)

Vulkaninsel (= Manam)

Dampier-

Insel

(= Karkar)

\section{Dampier-}

Insol

Südost-

Britisch-

Nou-Guinea

(Collingwood-

Bai)

D'Entre-

casteaux-

Inseln

(Fergusson $=$ Moratau)

Archipel

(Normanby

Insel),

Woodlark (1912). 
subsp. ap pin a (Fruhst.) Tenaris c. a., Fruh- Britischstorfer, Soc. Entom., v. 19, p. 129 (1904). - Id., Ent. Zeit. Guben, v. 18, p. 119 (1904). - Taenaris c. a., Fruhstorfer, Wien. Ent. Zeit., v. 24, p. 125 (1905). - H. Stichel, Gen. Ins., fasc. 36 , p. 46 (1906). - Fruhstorfer: A. Seitz, Großschmett. Frde, v. 9, p. 417 , t. 101 a (1911). - H. Stichel, Tierreich, Lief. 34 , p. 175 (1912).

? Drusilla catops, Ch. Oberthür, Ann. Mus. Civ. Stor. Natural. Genova, v. 15, p. 509 (1880). - Tryon, 2. Ann. Rep. Admin. Brit. N. Guinar, App. 5, sep. p. 7 (1890). Id.: J. P. Thomson, Brit. N. Guinea, App. 3, p. 251 (1892).

subsp. $t u r d u l$ a Fruhst. T. c. $t$., Fruhstor- Südwest-

fer, Ent. Rundschau, v. 31, p. 26 (1914). Neu-Guinea

\section{(Yule =}

Lolo-Insel)

subsp. mylaecha (Westw.) Drusilla m., IouisiadeWestwood, Trans. Ent. Soc. Iondon, n. ser., Archipel v. 1, p. 175 (1851). - (E. Doubleday \&) Westwood, Gen. Diurn. Lep., v. 2, Addit.; p. 533 (1852). - White, Macgillivray, Voy. Rattlesnake, v. 2, App., p. 390 , t. 4, f. 3, 4 (1852). - Westwood, Trans. Ent. Soc. London, n. ser., v. 4, p. 182 (1858). - Tenaris m., Herrich-Schäffer, Corr-Bl. Zool.-Min. Ver. Regensb., v. 19, p. 89 (1865). - IV. F. Kirby, Cat. Diurn. Lop., p. 118 (1871). - A. G. Butler, Proc. Zool. Soc. London, p. 279 (1874). - Drusilla m., J. G. Ward, Ins. abroad, p. 391, f. $334(1874)$. - Tenaris m., O. Staudinger (\& Schatz), Exot. Schmett., v. 1 , p. 192,198 (? T. catops var.) (distr. part.) (1887). - ? T. catops var. m., Tryon, 2. Ann. Rep. Admin. Brit. N. Guinea, App. 5, sep., p. 7 (1890). - Id.: J. P. Thomson, Brit. N, Guinea, App. 3, p. 251 (1892). Tuenaris c. m., Fruhstorfer, Wien. Ent. Zeit., v. 24, p. 121 (1905). - H. Stichel, Gen. Ins., fasc. 36, p. 45 (1906). - Fruhstorfer: A. Seitz, Großschmett. Erde, v. 9, p. 418 (1911). - H. Stichel, Tierreich, Lief. 34, p. 172 (1912), - Drusilla m., N. D. Riley \& Gabriel, Cat. Type Specim. Rhop. Brit. Mus., pars 1 , p. 38 (1924).

subsp. rosseliana Rothsch. T. c. r., W. Rossel-Ins. Rothschild, Nov. Zool., v. 23, p. 303 (1916).

subsp. $n i c a s i u s$ (Frulst.) T. c. n., Frub- Patria storfer, Wion. Ent. Zeit., v. 24, p. 86, 121 ignota (1905). - H. Stichel, Gen. Ins., fasc. 36, p. $46(1906)$. Fruhstorfer: $\Lambda$. Seitz, Großschmett. Erde, v. 9, p. 418 (1911). H. Stichel, Tierreich, Lief. 34, p. 178 (1912). subsp. nova $T$. catops, G. A. Waterhouse \& DarnleyLyell, Butterfl. Australia, p. 49, t. 38, f. Insel 781 (? subsp.) (1914). 


\section{Cohors e. Myopiformes}

H. Stichel, Gen. Ins., fasc. 36, p. 47 (1906). - Id., Tierreich, Lief. 34, p. 179 (1912).

tainia (Fruhst.) Tenaris t., Fruhstorfer, Ins.-Börse, Nord-Ostv. 22 , p. $36(1905)$.

Neu-Guinea

Ta enaris t., H. Stichel, Gen. Ins., fasc. 36 , p. 47 (1906). - Fruhstorfer: A. Seitz, Großschmett. Erde, v. 9, p. 422 (1911). - H. Stichel, Tierreich, Lief. 34, p. 179 (1912).

subsp. $t a$ in ia (Fruhst.) Tenaris $t$., Fruh- Ostliches storfer, ut antea (1905). - Taenaris t., T. Mitteldioptrica t., id., Wien. Ent. Zeit., v. 24, p. Neu-Guinea 36, 119 ; p. 124 (1905). - T. t. t., H. Stichel, Gen. Ins., fasc. 36, p. 47 (1906). - Fruhstorfer: A. Seitz, Großschmett. Erde, v. 9, p. 422 , t. 101 b (1911). - I. Stichel, Tierreich, Ijief. 34 , p. 180 (1912).

subsp. a nnella Stich. T. t. a., H. Stichel, NordostGen. Ins., fasc. 36, p. 47 (1906). - Fruh- Neu-Guinea storfer: A. Seitz, Großschmett. Erde, v. 9, p. 422 (1911). - It. Stichel, Tierreich, Lief. 34 , p. 180 , f. 31 (1912).

(Geelvink-

Humboldt-

Bai)

perplexus Rothsch. T. p., W. Rothschild, Nov. Zool., Südwestv. 23, p. 305 (1916).

Neu-Guinea (Triton-Bai)

Rothsehildi (Gr. Smith) Tenaris $r$., Grose Smith, Neu-Guinea Nov. Zool., v. 1, p. 359 (1894). - Taenaris r. + T. vahnesi, Fruhstorfer, Wien. Ent. Zeit., v. 24, p. 125, 124, 126 (1905). - H. Stichel, Gen. Ins., fasc. 36 , p. 48,47 (1906). - T. r., Fruhstorfer: A. Seitz, Großschmett. Ende, v. 9, p. 421 (1911). - H. Stichel, Tierreich, Lief. 34, p. 181 (1912).

subsp. Rothschildi (Gr. Smith) T'enaris $r$., Grose Smith, ut antea (1891). - Grose Smith \& W. F. Kirby, Rhop. Exot., v. 2, t. Tenaris 2, f. 1, 2 ( $\left.\mathrm{O}^{7}\right)$, f. 3,4 (O), p. 5; text ad t. Tenaris 3, p. 9 (1894 \& 95). Röber, Ent. Zeit. Stettin, v. 64, p. 342 (1903). - Taenaris r., Fruhstorfer, Wien. Ent. Zeit., v. 24, p. 119 (1905). - H. Stichel, Gen. Ins., fasc. 36, p. 48 (1906). - Strand, Mitt. Zool. Mus. Berlin, v. 5, p. 470 (1911); id., Archiv f. Naturgesch. 1912. A. 11. p. 65. T. $r . r_{.}$, Fruhstorfer: A. Seitz, Großschmett. Erde, v. 9, p. 421 (1911). - T. r. $r$. forma principalis, H. Stichel, Tierreich, Lief. 34, p. 182 (1912). - T. r., Eecke, Nova Guinea, v. 13, Zool., livr. 1, p. 63 (1915).

Tenaris dimona var. aut ab. (non Drusilla d. Hewitson), Grose Smith, Nov. Zool., v. 1, p. 360 (1894). - T. d. ab., O. Staudinger, Deutsch. Ent. Zoit. Lep., v. 7, p. 109 (1894).

forma pelagia (Fruhst.) Tenaris wahnesi Typ.: p., Fruhstorfer, Iint. Zeit. Guben, v. 18, p. Humboldt118 (1904). - Id., Soc. Entom., v. 19, p. Bai, Neu-Guinea (HumboldtBai) ? Finschküste

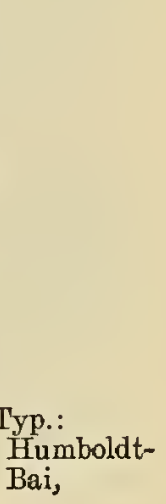


138 (1905). - Taenaris w. p., id., Wien.

Ent. Zeit., v. 24, p. 66, 119, 124 (1905). -

H. Stichel, Gen. Ins., fasc. 36, p. 47 (1906). - T. rothschildi ,Form" p., Fruhstorfer: A. Seitz, Großschmett. Erde, v. 9, p. 421, t. 101 b (1911). - ? T. wahnesi p. (?), Strand, Mitt. Zool. Mus. Berlin, v. 5, p. 470 (1911). - $T$. rothschildi $r$. forma p., H. Stichel, Tierreich, Lief. 34, p. 183 (1912). - T. $r$. p., Eecke, Nova Guinea, v. 15, Zool., p. 37 (1924).

- subsp. Wa hnesi (Heller) Tenaris w., Heller, Ent. Nachr., v. 20, p. 372 (1894). - B.

Hagen, Jahrb. Nassau. Ver. Naturk., v. "50, p. 81 (1897). - Tacnaris w, T. w. w., Fruhstorfer, Wien. Ent. Zeit., v. 24, p. 64, 119; p. 124 , t. 2 (larva) (1905). - $T . w . w .$, H. Stichel, Gen. Ins., fasc. 36, p. 47 (1906). - Taenaris rothschildi $w$, Fruhstorfer: A. Seitz, Großschmett. Erde, v. 9, p. 421 (1911). - T. w., T. w. w., Strand, Mitt. Zool. Mus. Berlin, v. 5, p. 470 (tainides Fruhst.?), (1911). - T. rothschildi $w$. forma principalis H. Stichel, Tiorreich, Iief. 34, p. 185 , f. 33 (1912). — T. r. w., Strand, Árch. Naturg., v. 78, 1912, Abt. A, Heft 11, p. 65 77 (1913).

forma candida (Fruhst.) Tenaris wahnesi Typ.: ab. c., Fruhstorfer, Soc. Entom., v. 19, p. 138 (1904). - Taenaris $w$. ab. c., id., Wien. Ent. Zeit., v. 24 , p. 65,119 (1905). - $T$. $w$. $w$. forma c., $\mathrm{H}$. Stichel, Gen. Ins., fasc. 36, p. 47 (1906). - T. rothschildi c., Fruhstorfer: A. Seitz, Großschmett. Erde, v. 9, p. 421 (1911). - T. r. wahnesi forma c., H. Stichel, Tierreich, Lief. 34, p. 187 (1912). - T. w. forma c., Fruhstorfer, Deutsch. Ent. Zeit. Iris, v. 24, p. 53 (1924).

forma tainides (Fruhst.) Tenaris wahnesi Typ.

$q$ ab. $t$.; Fruhstorfer, Soc. Entom., v. 19, p. 138 (1904). - Taenaris $w$. ab. t., id., Wien. Ent. Zeit, v. 24, p. 65, 119 (1905). - T. $w$. $w$. forma $t ., \mathrm{H}$. Stichel, Gen. Ins., fasc. 36 , p. $47(1906)$. - $T$. rothschildi $w$., , Form" t., Fruhstorfer: A. Seitz, Großschmett. Erde, v. 9, p. 422 (1911). - T. r. $w$. forma t., H. Stichel, Tierroich, Lief. 34, p. 187 (1912).

forma hadina (Fruhst.) Tenaris wahncsi ab. Typ.: h., Fruhstorfer, Soc. Entom., v. 19, p. 139 Ehemalig. (1904). - Taenaris w. ab. h., Fruhstorfer, Wien. Ent. Zeit., v. 24, p. 65, 119 (1905). $T$. $w$. $w$. forma $l_{\text {. }}$ H. Stichel, Gen, Ins., fasc. 36, p. 47 (1906). - T. rothschildi $w$. "Form" h., Fruhstorfer: A. Seitz, Großschmett. Erde, v. 9, p. 422 (1911). - T. r. w. forma $h$., H. Stichel, Tierreich, Lief. 34, p. 187 (1912).

Deutsch-

Neu-Guinea
? Finsch-

küste

Ostliches

Neu-Guinea

Whemalig.

Deutsch-

Neu-Guinea

(Stephansort)

Ehenalig.

Deutsch-

Neu-Guinea 
forma $g$ e $m$ mat a Stich. T. r. $w$. forma g., Typ.:

H. Stichel, Gen. Ins., fasc. 36, p. 47 (1906). Ehemalig.

- T. r. w. „Abweichung" g., Fruhstorfer:

A. Seitz, Großschmett. Erde, จ. 9, p. 422 (1911). - T. r. w. forma g., H. Stichel, Tierreich, Lief. 34, p. 186 (1912).

Wahnesi-trioculatus (Thiele) Tenaris wahnesi-trioculatus, $\mathbf{H}$. Thiele, Ins.-Börse, จ. 23, p. 40 (1906).

forma $s u b q u a d r i o c e l l a t a$ Strand $T$. $w$. Typ.: 8. Strand, Mitt. Zool. Mus. Berlin, v. 5, p. Ehemalig. 470 (1911). - T. rothschildi $w$. "Form" s., DeutschFruhstorfer: A. Seitz, Großschmett. Erde, v. 9, p. 449 (1911). - T. r. forma s., H. Neu-Guinea Stichel, Tierreich, Lief. 34, p. 187 (1912). - T. w. s., Strand, Bull. Soc. Zool. France, y. 51, p. $402(1926)$.

forma $q u a d r i o c e l l a t a$ Strand T. w. q., Typ.: Strand, Mitt. Zool. Mus. Berlin, v. 5, p. 470 Ehemalig. (1911). - T. rothschildi $w$. "Form" q., Fruhstorfor: A. Seitz, Großschmett. Erde, v. 9, p. 449 (1911), - T. r. w. forma $q ., \mathrm{H}$. Stichel, Tierreich, Lief. 34, p. 187 (1912). T. $w$. q., Strand, Bull. Soc. Zool. France, v. 51 , p. 402 (1926).

forma prodiga Fruhst. T. r. w. p., Fruh- Typ.: storfer: A. Seitz, Großschmett. Erde, v. 9, Ehemalig. p. 421,422 (1911).

Deutsch-

Neu-Guinea

T. $r$. forma quadriocellata, H. Stichel, Neu-Guinea Tierreich, Lief. 34, p. 187 (1912).

forma $l a c r i m a n s$ Fruhst. T. $w$. $l$., Fruh- Typ.: storfer: $\Lambda$. Seitz, Großschmett. Erde, v. 9, Ehemalig. p. 422 (1911). - $T$. rothschildi $w$. forma $l$., DeutschH. Stichel, Tierreich, Lief. 34, p. 187 (1912), Neu-Guinea

forma sidus Fruhst. T. w. s,, Fruhstorfer: Typ.: A. Seitz, Großschmett. Erde, v. 9, p. 422 Ehemalig. (1911). - T. rothschildi $w$. forma s., H. DeutschStichel, Tierreich, Lief. 34, p. 187 (1912). Neu-Guinea

subsp. $r e d u c t a$ Rothsch. $T$. rothschildi re- Vulkan-Insel ducta, W. Rothschild, Nov. Zool., v. 22, p. (Manam) 202 (1915).

subsp. merana (Fruhst.) Tenaris $m$., Fruh- Südstorfer, Ent. Zeit. Guben, v. 18, p. 118 (1904). Holländisch- Id.. Soc. Entom., v. 19, p. 138 (1904). Neu-Guinea - Taenaris wahnesi m., id., Wien. Ent. (Kajumera Zeit., จ. 24, p. 66, 119, 124 (1905). - H. Bai) Stichel, Gen. Ins., fasc. 36, p. 43 (1906). $T$. rothschildi m., Fruhstorfer: A. Seitz, Großschmett. Erde, v. 9, p. 421, t. $101 \mathrm{~b}$ (1911). - H. Stichel, Tierreich, Lief. 34, p. $183(1912)$.

subsp. 'V a $n$ h a a s te $r$ ti Hulst. T. r. v., Hul- Südstaert, Ann. Mag. Nat. Hist., ser. 9, v. 15, Holländischp. 445 (1925).

Neu-Guinea (Digul) 
subsp. ma ne t a Hulst. T. $r$. m., Hulstaert, SüdAnn. Mag. Nat. Hist., ser. 9, v. 11, p. 184 Holländisch(1923).

Neu-Guinea

(Merauke-

Fluß)

subsp. ans una (Fruhst.) Tenaris wahnesi Geelvink-

a., Fruhstorfer, Ent. Zeit. Guben, v. 18, p. Bai

118 (1904). - Id., Soc. Entom., v. 19, p. (Jobi)

138 (1905). - Taenaris w. a., id., Wien.

Ent. Zeit., v. 24, p. 66, 119, 124 (1905).-

H. Stichel, Gen. Ins., fasc. 36, p. 47 (part.) (1906). - T. rothschildi a., Fruhstorfer: A. Seitz, Großschmett. Erde, v. 9, p. 421 (1911). - T. r. a. forma principalis H. Stichel, Tierreich, Lief. 34, p. 183 (part.), f. 32 (1912).

forma $r a f a$ e la (Fruhst.) Tenaris r., Fruh- Typ.: Jobi storfer, Soc. Entom., v. 19, p. 138 (ansuna ab. ?) (1905). - Taenaris wahnesi ansuna ab. r., id., Wien. Fnt. Zeit., v. 24, p. 66, 119 (1905). - T. w. a. forma r., H. Stichel, Gen. Ins., fase. 36, p. 47 (1906). - T. rothschildi a. "Farbenabweichung" $r$., Truhstorfer: A. Seitz, Großschmett. Erde, v. 9, p. 421 (1911). - T. r. a. forma a., II. Stichel, Tierreich, Lief. 34, p. 184 (1912).

subsp. se naria Stich. T. wahnesi s., H. Salwati,

Stichel, Gen. Ins., fasc. 36, p. 48, t. 6, f. 16 Sorrong (०) (1906). - T. rothschildi s., Fruhstorfer: A. Seitz, Großschmett. Erde, Y. 9, p. 421 (1911). - H. Stichel, Tierreich, Lief. 34 , p. 184 (1912).

subsp. paralle u $_{8}$ Rothsch. T. r. p., W. Mrisol Rothschild, Nov. Zool., v. 23, p. 305 (1916).

dioptrica (Snell. v. Voll.) Drusilla d., Snellen v. Vollen- Neu-Guinea, hoven, Tijdschr. Ent., v. 3, p. 38 (1860). - Waigeu, Taenaris d. (part.) + T. timesias (part.), Salwati, Fruhstorfer, Wien. Ent. Zeit,, v. 24, p. 124, ? Molukken 125 (1905). - T. d., H. Stichel, Gen. Ins., fasc. 36 , p. 48 (1906). - Fruhstorfer: A. Seitz, Großschmett. Erde, v. 9, p. 422 (1911). - H. Stichel, Tierreich, Lief. 34, p. 187 (1912).

subsp. di o ptrica (Snell.v. Voll.) Drusillad., Snellen v. Vollenhoven, ut antea, t. 2, f. 2, 3 (1860). Tenaris $d$., Herrich-Schäfer, Corr.-Bl. Zool.-Min. Ver. Regensb., v. 19, p. 89 (1865). - W. F. Kirby, Cat. Diurn. Lep., p. 118 (1871). - T. artemis ab. (var. ?) d., O. Staudinger (\& Schatz), Exot. Schmett., v. 1, p. 192,197 (1887). - ? T. d., A. Pagenstecher, Abh. Sonckenb. Naturf. Ges., v. 23, p. 405 (1894). - Taenaris d., T. d. d., Fruhstorfer, Wien. Ent. Zeit., v. 24 , p. 59, 119, 124 (1905). - T. d. d., H. Stichel, Gen. Ins., fasc. 36, p. 48 (1906). T. d., Eruhstorfer, Deutsch. Ent. Zeit. Iris, v. 24 , p. 54, t. 2 , f. 4 (Organ. copul.) (1910).

Nördliches (Holländ.) Neu-Guinea (Sorrong), ? Halmahera (sec. Pagenstecher) 
- T. d. d. forma principalis, H. Stichel, Tierreich, Lief. 34, p. 188 (1912). - W. Rothschild (\& Durrant), Rep. Brit. Ornithol. Un. Exp. Dutch N. Guinea 2, Nr. 15, p. 19 (1915).

forma licinia (Fruhst.) Tenaris hyper- Typ.:

bolus l., Fruhstorfer, Ent. Zeit. Guben, v. 18, p. 118 (patria errans) (1904). - "Taenaris dioptrica forma $l$., id., Wien. Ent. Zeit., v. 24, p. 59, 119 (1905). - T. d. d. forma $l$., H. Stichel, Gen. Ins., fasc. 36, p. 48 (1906). - T.d.d. "Form" i., Fruhstorfer: A. Seitz, Großschmett. Ende, v. 9, p. 422 (1911). T. $d . d$. forma l., H. Stichel, Tierreich, Lief. 34, p. 189 (1912).

PDrusilla dioptrica, Ch. Oterthür, Ann. Mus. Civ. Stor. Natural. Genova, v. 15, p. 512 (Halmahora, Teruate) (1880).

subsp. timesias (Kirsch) Drusilla $t$., Inseln der Kirsch, Mitt. Zool. Mus. Dresden, v. 1, p. Geelwink121 (1877). - Tenaris artemis ab. t., 0 . Staudinger (\& Schatz), Exot. Schmett., v. 1, p. 192, 197 (distr. part.) (1887). Taenaris t., T. t. t., Fruhstorfer, Wien. Ent. Zeit., v. 24, p. 67, 119, 125 (1905). - T. dioptrica $t ., \mathrm{H}$. Stichel, Gen. Ins., fasc. 36, p. 48 (1906). - Fruhstorfer: A. Seitz, Großschmett. Erde, v. 9, p. 422 (1911). T. $d$. $t$. forma principalis, H. Stichel, Tierreich, Lief. 34, p. 189 , f. 34 (1912).

forma aesculapus (Stauding.) Tenarishy-Typ.: Jobi perbolus var. (ab.?) a., O. Staudinger (\& Schatz), Exot. Schmett., v. 1, p. 192, 196 (1887). - Taenaris dioptrica $h$. ab. a., Fruhstorfer, Wien. Ent. Zeit., v. 24, p. 61, 119 (1905). - T. d: timesias forma a., H. Stichel, Gen. Ins., fasc. 36 , p. 48 (1906). $-T$. d. a., Fruhstorfer: A. Seitz, Großschmett. Erde, v. 9, p. 422 (timesias, Albino) (1911). - T. d. $t$. forma $a$., H. Stichel, Tierreich, Lief. 34, p. 190 (1912).

subsp. wattina (Fruhst.) Tenaris $d$. w., Salwati Fruhstorfer, Ent. Zeit. Guben, v. 18, p. 119 (1904). - Taenaris d. $w_{\text {., }}$ id., Wien. Ent. Zeit., v. 24, p. 60,119 (1905). - H. Stichel, Gen. Ins., fasc. 36, p. 49 (1906). Fruhstorfer: A. Seitz, Großschmett. Erde, v. 9 , p. 422 (1911). - H. Stichel, Tierreich, Lief. 34, p. 190 (1912).

- subsp. a mit aba (Fruhst.) Tenaris 'hyper- Waigeu bolus a., Fruhstorfer, Ent. Zeit. Guben, v. 18, p. 118 (1904). - Taenaris dioptrica a., id., Wien. Ent. Zeit., v. 24, p. 60, 119 (1905). - H. Stichel, Gen. Ins., fase. 36, p. 49 (1906). - Fruhstorfer: A. Seitz, Großschmett. Erde, v. 9, p. 422, t. 101 b (1911). - H. Stichel, Tierreich, Lief. 34, p. 190 (1912). 
? Hyades (Tenaris) dioptrica, Capronnier, Ann. Soc. Ent. Belgique, v. 30, p. 6 (1886).

Tenaris hyperbolus (non Drusilla $h$. Kirsch), O, Staudinger (\& Schatz), Exot. Schmett., v. 1, p. 196 (1887).

subsp. orientalis Rothsch. T. d. o., W. Nordost-

Rothschild, Nov. Zool., v. 23, p. 304 (1916). Neu-Guinea

(Eriba,

Astrolabe-

Bai)

subsp. on esimides (Fruhst.) Tenaris hy- Westperbolus o., Fruhstorfer, Ent. Zeit. Guben, Holländischv. 18, p. 118 (1904). - Taenaris dioptrica o., id., Wien. Ent. Zeit., v. 24, p. 60, 119, 124 (1905). - H. Stichel, Gen. Ins., fasc. 36, p. 48 (1906). - Fruhstorfer: A. Soitz, Großschmett. Erde, v. 9, p. 422 (1911). H. Stichel, Tierreich, Lief. 34, p. 190 (1912). subsp. Rileyi Hulst. T. d. $r$., Hulstaert, SüdAnn. Mag. Nat. Hist., ser. 9, v. 15. p. 444 Holländisch(1925).

Neu-Guinea

(Kapaur,

Itna-Bai)

Neu-Guinea

(Digul)

subsp. onesimus (Butl.) Tenaris o., A. Neu-Guinea

G. Butler, Proc. Zool. Soc. London, p. 468

(1877). - Taenaris dioptrica o., Fruhstorfer, Wien. Ent. Zeit., v. 24, p. 64, 119, 124 (1905). - H. Stichel, Gen. Ins., faso. 36, p. 48 (1906). - Fruhstorier: A. Seitz, Großschmett. Ende, v. 9, p. 422 (1911). - H. Stichel, Tiorroich, Lief. 34, p. 191 (1912).Tenaris o., N. D. Riley \& Gabriel, Cat. Type Specim. Rhop. Brit. Mus., pars 1, p. 41 (1924).

eyclops (Stauding.) Tenaris c., O. Staudinger, NordostDeutsch. Ent. Zeit. Lep., v. 6, p. 367 (1893). Neu-Guinea - Heller, Ent. Nachr., v. 20, p. 375 (1894). (Kaiser-Wil- B. Hagen, Jahrb. Nassau. Ver. Naturk., v. 50, p. 81 (1897). - Taenaris timasias c., Fruhstorfer, Wien. Ent. Zeit., v. 24, p. 68, 119, 125 (1905). - T. c., H. Stichel, Gen. Ins., fasc. 36, p. 49 (1906). - T. c. forma c., Fruhstorfer, Deutsch. Int. Zeit. Iris, v. 24, p. 53 , t. 2, f. 3 (Organ. copul.) (1910). - T. c. c., id.: A. Seitz, Großschmett. Erde, v. 9, p. 423 (1911). - T. c. forma principalis, H. Stichel, Tierreich, Lief. 34, p. 191 (1912).

forma agasta Stich. T. c. forma a., H. Typ.:

Stichel, Gen. Ins., fasc. 36 , p. 49 , t. 6 , f. 17 Ehemalig.

(1906). - Fruhstorfer, Deutsch. Ent. Zeit. Dentsch-

Iris, v. 24 , p. 53 (1910). - T. c. ,Form" Neu-Guinea

a., Fruhstorfer: A. Seitz, Großschmett. Erde, v. 9 , p. 191 (1911) - T. c. forma a., H. Stichel, Tierreich, Lief. 34, p. 192 (1912).

forma a ga peth a (Fruhst.) Tenaris timesias Typ.:

(?) agapethus, Fruhstorfer, Soc. Entom., v. Neu-Guinea 
19, p. 138 (1904). - Taenaris t. a., T. $t$. forma $a$. id., Wien. Ent. Zeit.s v. 24, p. 67, 119, 125 (1905). - T. cyclops forma a., H. Stichel, Gen. Ins., fasc. 36, p. 49 (1906). - Fruhstorfer, Deutsch. Ent. Zeit. Iris, v. 24, p. 53 (1910). - T. c. "Form" a., id.: A. Seitz, Großschmett. Erde, v. 9, p. 423,.t. $101 \mathrm{c}$ (1911). - H. Stichel, Tierreich, Lief. 34 , p. 192 (1912).

forma $F$ erdinandi (Fruhst.) Tenaris di- Typ.:

optrica f., Fruhstorfer, Soc. Entom., v. 19, Ehemalig. p. 138 (1904). - Taenaris cyclops ab. $f$, Deutschid., Wien. Ent. Zeit., v. 24, p. 70; T. ti- Neu-Guinea mesias c. ab. f., id., l. c., p. 119 (1905). $T$. c. forma $f ., H$. Stichel, Gen. Ins., fasc. 36, p. 49 (1906). - T. c. var. f., Fruhstorfer, Deutsch. Fnt. Zuit. Iris, v. 24, p. 53 (1910). - T. c. "Form" t., id.: A. Seitz, Großschmett. Erde, v. 9, p. 423 (1911). - T. c. forma $f, 3$. Stichel, Tierreich, Lief. 34, p. 192 (1912).

chionides (Godm. \& Salv.) Tenaris c., F. D. God- Neu-Guinea man \& O. Salvin, Proc. Zool. Soc. Lond., p. 611 (1880). - Taenaris c., Fruhstorfer, Wien. Ent. Zeit., v. 24, p. 125, 126 (1905). - H. Stichel, Gen. Ins., fasc. 36, p. 49 (1906). Fruhstorfer: A. Seitz, GroBschmett. Erde, v. 9, p. 424 (1911). - H. Stichel, Tierreich, Lief. 34, p. 192 (1912).

- subsp. chionides (Godm. \& Salv.) Tenaris c., F. D. Godman \& O. Salvin, ut antea (1880). - O. Staudinger (\& Schatz), Exot. Schmett., v. 1, p. 193, 198 (1887). - Taenaris c. c., Fruhstorfer, Wien. Ent. Zeit., v. 24 , p. 119,125 (1905). - H. Stichel, Gen. Ins., fasc. 36, p. 49 (1906). - $T$. c., Fruhstorfer: A. Seitz, Großschmett. Erde, v. 9, p. 424 (1911). - T. c. c. forma principalis, H. Stichel, Tierreich, Lief. 34, p. 193 (1912). - Tenaris c., N. D. Riley \& Gabriel, Cat. Type Specim. Rhop. Brit. Mus., pars 1, p. 14 (1924).

forma aroana (Fruhst.) Tenaris liubaryi a., Typ.: Fruhstorfor, Ins.-Börse, v. 18, p. 373 (1901).

- Id., Doutsch. Ent. Zeit. Lep., v. 14, 1901, p. 330 (1902). - Td., Berlin. Ent. Zeit,, v. 47, Sitz.-Ber., p. (20) (1902). - Taenaris chionides a., id., Wien. Ent. Zeit., v. 24, p. 120 (1905). - T. c. c. forma a., $\mathrm{H}$. Stichel, Gen. Ins., fasc. 36, p. 49 (1906). T. c. kubaryi forma a., Fruhstorfer, Deutsch. Ent. Zeit. Iris, v. 24, p. 52 (Finschhafen), (1910). - T. c. $a_{.,}$id.: A. Seitz, Großschmett. Frde, v. 9, p. 424, 1111, t. 101c (1911 \& 27). - T. c. c. forma a., H. Stichel,

Tierreich, Lief. 34, p. 193 (1912).
- subsp. Kubaryi (Stauding.) Tenaris k., Ehemaliges

O. Staudinger, Deutsch. Ent. Zeit. Lep., v. Deutsch-

6, 1893, p. 365, t. 7, f. 2 (1894). - Id.) Nou-Guinea 
l. c., v. 7, p. 114 (part.) (1894). - B. Hagen, Jahrb. Nassau. Ver. Naturk., v. 50, p. 80 (1897). - Taenaris chionides k., Fruhstorfer, Wien. Ent. Zeit., v. 24, p. 120, 125 (1905). - H. Stichel, Gen. Ins., fasc. 36, p. 49 (1906). - T. c. k. forma k. Fruhstorfer, Deutsch. Ent. Zeit. Iris, v. 24, p. 52, t. 2, f. 1 (Organ. copul.) (1910). - T. c. k. forma principalis, H. Stichel, Tierreich, Lief. 34, p. 193 (1912).

forma eugen ia Fruhst. T. c. e., Fruhstor- Typ.:

fer: A. Seitz, Großschmett. Erde, v. 9, p. 424 , t. 101 c (1911). - T. c. Kubaryi forma e., Fruhstorfer, Deutsch. Ent. Zeit. Iris, v. 24 , p. 53 , t. 2 , f. 2 (Organ. copul.) (1910). - 1. c. c. forma e., H. Stichel, Tierreich, Lief. 34, p. 193 (1912).

forma $c y c l o p i d e \&$ Fruhst. $T$. chionides ku- Typ.:

baryi forma cyclopides, Fruhstorfer, Deutsch. Kaiser-

Ent. Zoit. Iris, v. 24, p. 53 (1910). - $T$. Wilhelms-

chionides cyclopides, Fruhstorfer: A. Seitz, Land

Großschmett. Erde, v. 9, p. 424 (1911). -

$T$. chionides $c$. forma cyclopides, H. Stichel, Thierreich, Lief. 34, p. 193 (1912).

T. $K u b a r y i, 0$. Staudinger, Deutsch. Ent. Zeit. Lop., У. 7, p. 114 (1894).

forma $a m b i g u a$ Stich. $T$. c. $k$. forma $a_{*}$, Typ.:

H. Stichel, Gen. Ins., fasc. 36, p. 49 (1906). Ehemal.

- Frulsstorfor, Deutsch. Ent. Zoit. Tris, v. Deutsch-

24, p. 52 (1910). - T. c. a., Fruhstorfer: A. Neu-Guinea

Seitz, Grobschmett. Erde, v. 9, p. 193 (1911).

- T. c. kubaryi forma a., H. Stichel, Tierreich, Lief. 34, p. 193 (1912).

myops (Feld.) Drusilla m., C. \& R. Felder, Wien. Neu-Guinea

Ent. Monatschr., v. 4, p. 109 (1860). - und

Taenaris m. (part.), Fruhstorfer, Wien. Ent. benachbarte

Zeit., v. 24, p. 61, 124, 126 (1905). - H. Inseln

Stichel, Gen. Ins., fasc. 36 , p. 49 (1906). -

Fruhstorfer: A. Seitz, Großschmett. Erde, v. 9 , p. 422 (1911). - II. Stichel, Tierreich, Liof. 34 , p. 193 (1912). - W. Rothschild, Nov. Zool., v. 23, p. 306 (1916).

subsp. my॰p\& (Feld.) Drusilla m., C. \& Aru-Inseln

R. Felder, ut antea, t. 1, f. 1 (1860). -

Tenaris m., Herrich-Schäffer, Corr.-Bl. Zool.-

Min. VVer. Regensb., v. 19, p. 89 (1865). -

Drusilla m., Oh. Oberthür, Ann. Mus. Oiv.

Stor. Natural. Genova, v. 12, p. 463 (1878).

- A. G. Butler, Ann. Mag. Nat. Hist., ser. 5 , v. 11, p. 402,411 (1883). $-T$. artemis ab. (an var.?) m., O. Staudinger (\& Schatz, Exot. Schmett., v. 1, p. 192, 197 (1887). Taenaris m. m., Fruhstorfer, Wion. Ent. Zeit., v. 24, p. 119 (1905). - H. Stichel, Gen. Ins., fasc. 36, p. 49 ' (1906). - Fruhstorfer: A. Seitz, Großschmett. Erde, v. 9, p. 422 (1911). - H. Stichel, Tierreich, Lief. 34 , p. 194, f. 35 (q) (1912). - W. 
Rothschild, Nov. Zool., v. 23, p. 306 (1916). - Fruhstorfer, Ent. Rundschau, v. 34 , p. 43 (1917).

? Drusilla mylaecha (non Westwood), Howitson, Proc. Zool. Soc. Iondon, p. 52 (1861).

$D$ rusilla selcne (non Westwood) + $D$. catops (non Westw.), Kirsch, Mitt. Zool. Mus. Dresden, จ. 1, p. 120 (1877).

subsp. praxedcs (Fruhst.) "Tenaris m. p., Salwati Fruhstorfer, Ent. Zeit. Guben, จ. 18, p. 119 (1904). - Taenaris m. p., id., Wien. Ent. Zeit., v. 24, p. 119, 124 (1905). - H. Stichel, Gen. Ins., fasc. 36, p. 50 (1906). - Fruhstorfer: A. Seltz, Großschmett. Erde, v. 9, p. 423 (1911). - H. Stichel, Tierreich, Lief. 34, p. 199 (error in cit.: praxedes, non praexedes, Fruhstorfer, 1911) (1912). - W. Rothschild, Nov. Zool., v. 23, p. 306 (1916).

subsp. misolens is Rothsch. T. myops $\dot{m i}$ - Misol solensis, W. Rothschild, Nov. Zool, v. 23, p. 305,306 (1916).

T. m. $p r a x c d e s$ (non Fruhstorfer), W. Rothschild, Nov. Zool., v. 22, p. 125 (1915).

subsp. errhephoria Fruhst. $T$. $m$. e., SüdostFruhstorfer, Deutsch. Ent. Zeit. Iris, v. 29, Neu-Guinea p. 92 (1915). - T. kirschi e., id., Ent. Rund- (Finschschau, v. 34, p. 43 (1917). (Subsp. ordin. hafen) dubiae.)

subsp. fergussonia (Fruhst.) Tenaris m. D'Entref., Fruhstorfer, Ent. Zeit. Guben, v. 18, p. 119 (1904). - Taenaris m. f., T. f., Fruhstorfer, Wien. Ent. Zeit., v. 24, p. 62, 119; t. 2 (1905). - T. m. f., H. Stichel, Gen. Ins., fasc. 36, p. 50 (1906). - Fruhstorfer: A. Seitz, Großschmett. Erde, จ. 9, p. 423, t. 101 c $(1911)$. - T. m. f. forma principalis, H. Stichel, 'lierreich, Lief. 34, p. 195 (1912). - T. m. f., W. Rothschild, Nov. Zool., v. 23, p. 306 (1916). - Fruhstorfor, Entom. Rundschau, v. 34, p. 43 (1917).

Drusilla myops, F. D. Godman \& 0 . Salvin, Proc. Zool. Soc. London, p. 645 (1878). - ? Tenaris m., Tryon: J. P. Thomson, Brit. N. Guinea, App. 3, p. 258 (1892).

subsp. miscus Fruhst. T. myops miseus, Fruhstorfer, Wien. Ent. Zeit., จ. 24, p. 62, 119 (1905). - T. myops fergussoniu forma miscus, H. Stichel, Gen. Ins, fasc, 36, p. 50 (1906). - T. myops miscus, Fruhstorfer: A. Seitz, Großschmett. Erde, v. 9, p. 423 (1911). - T. myops fergussonia forma miscus, $H$. casteauxInseln (Fergusson, Mainland)

Stichel, Tierreich, Lief. 34, p. 196 (1912). - T. myops miscus, W. Rothschild, Nor. Zool., v. 23, p. 306 (1916).

? Tenaris $K i r s c h i$ (non Staudinger), W. F. Kirby, Ann. Mag. Nat. Hist., sor. 6, v. 3, p. 160 (1889).

IouisiadeArchipel (Normanby, GoodenoughInsel) 
subsp. Verbeeki (Fruhst.) Tenaris v., Südost-

Fruhstorfer, Soc. Entom., v. 19, p. 129 (1904). Neu-Guinea

- Taenaris kirschi forma v., T. myops v., (Milne Bai)

Fruhstorfer, Wien. Ent. Zeit., v. 24, p. 63, 191; T. v., t. 1 (1905). - T. myops mailua forma v., H. Stichel, Gen. Ins., fasc. 36, p. 50 (1906). - T. myops v., Frulustorfer: $\Lambda$. Seitz, Großschmett. Erde, v. 9, p. 423 (mailua, Zustandsform) (1911). - T. myops mailua forma $v ., H$. Stichel, Tierreich, Lief. 34, p. 197 (1912). - T. myops v., W. Rothschild, Nov. Zool., v. 23, p. 306 (1916). $T$. Firschi $v$., Fruhstorfor, Entom. Rundschau, v. 34, p. 43 (1917).

Kirschi (Stauding.) Tenaris k., O. Staudinger (\& Neu-Guinea, Schatz), Exot. Schmett., v. 1, p. 193, 197 D'Entre(1887). - Taenaris k., W. Rothschild, Nov. Zool., v. 23, p. 306 (1916).

T. myop 8 (non Drusilla m. Felder) (part.), Fruhstorfer, Wien. Ent. Zeit., v. 24, p. 124, 126 (1905). - H. Stichel, Gen. Ins., fasc. 36, p. 49 (1906). - Fruhstorfor: A. Seitz, Großschmett. Erde, v. 9, p. 422 (1911). H. Stichel, Tierreich, Lief. 34, p. 193 (1912). subsp. $K i r s c h i$ (Stauding.) Tenaris k., O. BritischStaudinger, ut antea (1887). - ? Grose Smith, Nov. Zool.,, v. 1, p. 359 (1894). Fürbringer (\& A. Pagenstecher); Semon, Zool. Forschungsreis., Denkschr. Med. Naturw. Ges. Jena, v. 8, p. (78) 238 (1895). - Taenaris myops k., Fruhstorfer, Wien. Ent. Zoit., v. 24, p. 63, 119, 124 (1905). H. Stichel, Gen. Ins., fasc. 36, p. 50 (1906). - Fruhstorfor: A. Seitz, Großschmett. Erde, v. 9 , p. 423 (1911). - H. Stichel, Tierreich, Lief. 34, p. 195, f. 37 (1912). - $T$. k. k., W. Rotlischild, Nov. Zool., v. 23, p. 306 (1916). - Fruhstorfer, Ent. Rundschau, v. 34, p. 43 (1917).

subsp. errhiphoria (non T. myops errhe- Yulephoria), Fruhstorfer, Deutsch. Ent. Zeit. Iris, (Lolo-)Insel v. 29, p. 91 (1915). - Id., Ent. Rundschau,

v. 34, p. 43 (1917).

subsp. convergens Rothsch. T. hirschi Fergusson

(laps. typogr.) c., T. kirschi c., W. Rothschild, Nov. Zool., v. 306,307 (1916). - $T$. k. c.. Fruhstorfer, Ent. Rundschau, v. 34, p. 43 (1917).

subsp. o c cidentalis Rothsch. T. k. o., W. Kapaur

Rothschild, Nov. Zool., v. 23, p. 306, 307

(1916). - Fruhstorfor, Fnt. Rundschau, v. 34 , p. 43 (1917).

subsp. interfa $u$ n $u$ \& Rothsch. T. k. i., W. NordwestRothschild, Nov. Zool., v. 23, p. 306, 307 Neu-Guinea (1916). - Fruhstorfer, Ent. Rundschau, v. (Humboldt34 , p. $43(1917)$.

? Tenaris kirschi, Grose Smith, Nov. Bai)

Zool., จ. 1, p. 359 (1894). 
mailua (Gr. Smith) T. m., Groso Smith, Nov. Zool., Südliches v. 4, p. 313 (1897). - Taenaris m., Fruh- Neu-Guinea, storfer, Wien. Ent. Zeit., v. 24, p. 125, 126 Rossel(1905). - W. Rothschild, Nov, Zool., v. 23, p. Insel 306 (1916).

T. myops (non Drusilla m. Folder) (part.), H. Stichel, Gen. Ins., fase. 36, p. 49 (1906). - Fruhstorfer: A. Seitz, Großschmett. Erde, v. 9, p. 422 (1911). - H. Stichel, Tierreich, Lief. 34, p. 193 (1912).

subsp. mailua (Gr. Smith) Tenaris m., Gebirge Grose Smith, ut antea (1897). - Grose SüdostSmith (\& W. F. Kirby), Rhop. Exot, จ. Neu3, t. Tenaris 5, f. 1,2 , p. 17 (1898). Taenaris m. m., Fruhstorfer, Wien. Ent. Zeit., v. 24, p. 119,125 (1905). - T. myops mailua, ( $T$. eichhorni Stauding. MS), H. Stichel, Gen. Ins., fasc. 36, p. 50 (1906). Guineas (Hinterland d. Milne- Fruhstorfer: A. Seitz, Großschmett. Erde, v. 9, p. 423 (1911). - T. myops mailua forma principalis, H. Stichel, Tierreich, Lief. 34, p. 196 (part.), f. 36 (Q) (1912). - T. mailua m., W. Rothschild, Nov. Zool., v. 23, p. 306 (1916). - Fruhstorfer, Ent. Rundschau, v. 34 , p. 44 (1917).

- subsp. littoralis Rothsch. T. mailua $l$., SüdostW. Rothschild, Nov. Zool., v. 23, p. 306, 307 (1916). - Fruhstorfer, Ent. Rundschau, v. 34, p. 44 (1917).

Neu-Guinea, Küstenzone (Milne Bai)

subsp. Ros s eli Fruhst. T. mailua r., Fruh- Rossel-Insel storfer, Wien. Fint. Zeit., v. 24, p. 65, 119 (1905). - T. myops r., H. Stichel, Gen. Ins., fasc. 36, p. 50 (1906). - Fruhstorfer: A. Seitz, Großschmett. Erde, v. 9, p. 423 (1911). - H. Stichel, Tierreich, Lief. 34 , p. 199 (1912). - T. mailua $r_{.}$, W. Rothschild, Nov. Zool., v. 23, p. 306 (1916).

\section{Cohors f. Arfemidiformes}

H. Stichel, Gen. Ins., fasc. 36, p. 50 (1906). - Id., Tierreich, Lief. 34, p. 199 (1912).

hyperbola (Kirsch) Tenaris hyperbolus, Kirsch, HolländischMitt. Zool. Mfus. Dresden, v. 1, p. 122 (1877). Neu-Guinea - Taenaris hyperbola, H. Stichel, Gen. Ins., fasc. 36 , p. 50 (1906). - T. hyperbolus, Fruhstorfer: A. Seitz, Großschmett. Erde, v. 9, p. 416 (1911). - T. hyperbola, $\mathrm{H}$. Stichel, Tierreich, Lief. 34, p. 199 (1912). - T. hyperbolus, Eecke, Nova Guinea, v. 13, Zool., livr. 1, p. 68 (1915).

subsp. hy perbola (Kirseh) Tenaris hyper-Geelwinkbolus, Kirsch, ut antea (1877). - T. h., O. Bai (Jobi) Staudinger (\& Schatz), Exot. Schmett., v. 1, p. 192 (non p. 196: = T. dioptrica amitaba, conf. Fruhstorfer, 1905) (1887). - Taenaris dioptrica $h$. , Fruhstorfer, Wien. Ent. Zeit., v. 24, p. 61, 119 (1905). $-T$. 
hyperbola, H. Stichel, Gen. Ins., fasc. 36, p. 50 (1906). - T. hyperbolus, Fruhstorfer: A. Seitz, Großschmett. Erde, v. 9, p. 416 (1911). - T. hyperbola forma principalis, H. Stichel, Tierreich, Lief. 34, p. 199,200, f. 33 (1912).

- subsp. a uto mo la (Kirseh) Tenaris automo- Nord-

lus, Kirsch, Mitt. Zool. Mus. Dresden, v. Holländisch-

1, p. 122 (1877). - T. h. ab. automolus Neu-Guinea, (part.), O. Staudinger (\& Schatz), Exot. Salwati

Schmett., v. 1, p. 192, 196 (1887). - Taenaris dioptrica a., Fruhstorfer, Wien. Ent. Zeit., v. 24, p. 61, 119, 124 (1905). T. hyperbola forma a., H. Stichel, Gen. Ins., fasc. $36, \quad$ p. 50 (1906). $-T$. hyperbolus automolus, Fruhstorfer: A. Seits, Großschmett. Erde, v. 9, p. 416 (Ortsrasse), t. $101 \mathrm{~d}$ (1911). - T. hyperbola forma automola, H. Stichel, Tierreich, Lief. 34, p. 200 (1912). - T. hyperbolus automolus, Eecke, Nova Guinea, v. 13, Zool. livr. 1, p. 68 (1915).

subsp. Ver ste egi Eecke T. hyperbolus v., ZentralEacke, Nova Guinea, v. 13, Zool., livr. 1, p. Nord68 (1915).

Neu-Guinea

subsp. concolor Hulst. T. hyperbolus c., SüdHulstaert, Ann. Mag. Nat. Hist., ser. 9, v. Holländisch15 , p. 444 (1925).

Neu-Guinea (Turai)

- artemis (Snell.-Voll.) Drusilla a., Snellen-Vollenhoven, Tijdschr. Ent., v. 3, p. 37 (1860) :Taenaris a., Fruhstorfer, Wien. Ent. Zeit., v. 24 , p. 125,126 (1905). - H. Stichel, Gen. Ins., fasc. 36, p. 51 (1906). - Fruhstorfer: A. Seitz, Großschmett. Erde, v. 9, p. 413 (1911). - H. Stichel, Tierreich, Lief. 34, p. 201 (1912). - G. A. Waterhouse \& Lyell, Butterfl.. Austral., p. 49 (1914).

forma sidus. Fruhst. T. a. (sidus Stich.), Fruhstorfer: A. Seitz, Großschmott. Ende, จ. 9, p. 414 (1911).

forma monop 8 Fruhst. T. $a$. (m.), Fruhstorfer: A. Seitz, Großschmett. Erde, v. 9, p. 414 (1911).

forma $l$ a crimans Fruhst. T. a. (l.), Fruhstorfer: A. Seitz, Großschmett. Erde, v. 9, p. 414 (1911).

forma o pulenta Fruhst. T. a. (opulenta Stich.), Fruhstorfer: A. Seitz, Großschmett. Erde, v. 9, p. 414 (1911).

forma ge $m$ m a $t$ a Fruhst. T. a. (gemmata Stich.), Fruhstorfer: A. Seitz, Großschmett. Erde, v. 9, p. 414 (1911).

forma $R$ oth \& childi Fruhst. T. a. ab. r., (non T. r. Grose Smith), Fruhstorfer: A. Seitz, Großschmett. Ende, v. 9, p. 414, 415 (1911).

Neu-Guinea, benachbarte Inseln, LouisiadeArchipel, NordAustralien 
subsp. artemis (Snell.-Voll.) Drusilla a., NordwestSnellen-Vollenhoven, ut antea, t. 1, f. 1, 2 Nordost(1860). - Tenaris a., Herrich-Schäffer, Corr.-Bl. Zool.-Min. Ver. Regensb., v. 19, p. 89 (1865). - W. F. Kirby, Cat. Diurn. Lep., p. 118 (1871), - Drusilla selene var. a., Kirsch, Mitt. Zool. Mus. Dresden, v. 1, p. 120 (1877). - D. a., Oh. Oberthür, Ann. Mus. Civ. Stor. Natural. Genova, v. 15, p. 510 (part.) (1880). - Tenaris a., O. Staudinger (\& Schatz). Exot. Schmett., v. 1, p. 192, 196 (part.) (1887). - Drusilla a., P. O. T. Snellen, Tijdschr. Ent., จ. 32, p. 389 (1889). - Taenaris a., T. a. a., Fruhstorfer, Wien. Ent. Zeit., v. 24, p. 74, 121, 125 (1905). - T. a. a., H. Stichel, Gen. Ins., fasc. 36, p. 51 (part.) (1906). Fruhstorfer: A. Seitz, Großschmett. Erde, v. 9 , p. 414 (1911). - Tenaris a., Rebel, Verh. Zool.-Bot. Ges. Wien, v. 61, p. (28) (1911). - Taenaris a. a. forma principalis, H. Stichel, Tierreich, Lief. 34, p. 202 (cit. part.) (1912). - ? T. a. a., W. Rothschild (\& Durrant), Rep. Brit. Ornithol. Un. Exped. Dutch N. Guinea, 2, Nr. 15, p. 18 (1915). - T. a., Eecke, Nov. Guinea, v. 13, Zool., livr., 1, p. 68 (1915).

Drusilla cato ps (non Westwood), Kirsch, Mitt. Zool. Mus. Dresden, v. 1, p. 120 (1877).

? T. Staudingeri, Röber, Nova Guinea, v. 13, Zool., livr. 1, p. 17 (1915).

forma eleusina (Fruhst.) Tenaris a. (?) e., Fruhstorfer, Ins.-Börse, v. 21, p. 389 (1904). - Taenaris a. ab. e., id., Wien. Ent. Zeit., จ. 24, p. 76, 121 (1905). T. a. a. forma e., H. Stichel, Gen. Ins., fasc. 36, p. 51 (1906). - Frubstorfer: A. Seitz, Großschmett. Ende, v. 9, p. 414 (1911). - H. Stichel, Tierreich, Lief. 34, p. 202 (1912).

subsp. zenada (Fruhst.) Tenaris a. z., Fruhstorfer, Ins.-Börse, マ. 21, p. 389 (1904). - Taenaris a. z., id., Wien. Ent. Zeit., v. 24, p. 76, 121, 125 (1905). - Id.: A. Seitz, Großschmett. Erde, v. 9, p. 414 (1911).

? T. Staudingeri S., W. Rothschild (\& Durrant), Rep. Brit. Ornithol. Un. Exped. Dutch N. Guinea 2, Nr. 15, p. 19 (1915).

T. arte $m$ is a. (part.), H. Stichel, Gen. Ins., fasc. 36 , p. 51 (1906). - Id., Tierreich, Lief. 34, p. 202 (1912).

subsp. intermedians Hulst. T. a. inter- Südmedians, Hustaert, Ann. Mag. Nat. Hist., ser. 9, v. 15, p. $444(1925)$.

Typ.:

Neu-Guinea

(Sorrong,

Mamberano-

Fluß)

Sorrong

Südwest-

Holländisch-

Neu-Guinea

(Kapaur,

Kajumera-

Bai)

Holländisch-

Neu-Guinea

(Turai)

subsp. blan'dina (Fruhst.) Tenaris a. b., Jobi

Fruhstorfer, Ins.-Börse, v. 21, p. 389 (1904).

- Taenaris a. b., id., Wien. Ent. Zeit., v. 
24, p. 77, 121 (1905). - T. a. humboldti forma b., H. Stichel, Gen. Ins., fasc. 36, p. 51 (1906). — T. a. b., Fruhstorfer: A. Seitz, Großschmett. Erde, v. 9, p. 415 (1911). - T. a. humboldti forma b., H. Stichel, Tierreich, Lief. 34, p. 205 (1912).

- subsp. gisela (Fruhst.) Tenaris a.g., Fruh- Waigeu storfer, Ins.-Börso, v. 21, p. 389 (1904). Id., Wien. Ent. Zeit., v. 24, p. 74, 121; T. g., t. 1 (1905). - T. a. g., H. Stichel, Gen. Ins., fasc. 36, p. 51 (1906). - Fruhstorfer: A. Seitz, Großschmett. Erde, v. 9, p. 414 (1911). - T. a. g. forma principalis, H. Stichel, Tierreich, Lief. 34, p. 203 (1912).

Tenaris artemis (part.), O. Staudinger (\& Schatz), Exot. Schmett., จ. 1, p. 192, 196 (1887).

forma timesides Fruhst. T. a. ab. t., Typ.:

Fruhstorfer, Wien. Ent. Zeit., v. 24, p. 74, Waigeu 121 (1905). - T. a. g. forma $t ., \mathrm{H}$. Stichel, Gen. Ins., fasc. 36, p. 51 (1906). T. a. $t .$, Fruhstorfer: A. Seitz, Großschmett. Erde, v. 9, p. 414 (1911). - T. a. gisela forma $t$., H. Stichol, Tierreich, Lief. 34, p. 204 (1912).

subsp. cels a (Fruhst.) Tenaris a. c., Fruh- Salwati storfer, Ins.-Börse, v. 21, p. 389 (1904).

Trenaris a. c., id., Wien. Ent. Zeit., v. 24, p. 75, 121 (1905). - H. Stichel, Gen. Ins., fasc. 36, p. 51 (1906). - Fruhstorfer: A. Seitz, Großschmett. Erde, v. 9, p. 414, t. $101 \mathrm{~d}$ (1911). - H. Stichel, Tierreich, Lief. 34 , p. 205 (1912).

? Drusilla artemis, Ch. Oberthür, Ann. Mus. Civ. Stor. Natural. Genova, v. 15, p. 510 (part.) (1880). - Tenaris a., O. Staudinger (\& Schatz), Exot. Schmett., v. 1, p. 192, 196 (part.) (1887).

subsp. ziada (Fruhst.) Tenaris a.z., Fruh- Misol storfer, Ins.-Bölse, v. 21, p. 389 (1904).

Taenaris a. z., id., Wien. Ent. Zeit., v. 24, p. 76, 121 (1905). - H. Stichel, Gen. Ins., fasc. 36, p. 51 (1906). - Fruhstorfer: A. Seitz, Großschmett. Ende, v. 9, p. 414, t. $101 \mathrm{~d}$ (1911). - H. Stichel, Tierreich, Lief. 34, p. 203 (1912). - W. Rothschild, Nov. Zool., v. 22, p. 125 (1915).

subsp, myopina (Fruhst.) Tenaris a. m., Aru-Inseln Fruhstorfer, Ins.-Börse, v. 21, p. 389 (1904). - Taenaris a. m., id., Wien. Ent. Zeit., $\vee$. 24, p. 74, 120 (1905). - H. Stichel, Gen. Ins., fasc. 36 , p. 51, t. 6 , f. 18 (1906). Fruhstorfer: A. Seitz, Großschmett. Erde, v. 9, p. 414 (1911). - H. Stichel, Tierreich, Lief. 34, p. 203 (1912).

Drusilla artemis, Ch. Oberthür, Ann. Stor. Natural. Genova, v. 15, p. 510 (part.) (1880). - Tenaris urania var. a., A. Pagen- 
stecher, Jahrb. Nassau. Ver. Naturk., v. 37, p. 178 (1884). - T. a., Ribbe, Corr.-Bl. Ent. Ver. Iris, v. 1, p. 82 (1886). - - O. Staudinger (\& Schatz), Exot. Schmett., v. 1, p. 192, 196 (part.) (1887). - A. Pagenstecher, Abh. Senckenb. Naturf. Ges., v. 33, p. 411 (1911).

subsp. $H u m b$ old $t i$ (Fruhst.) Tenaris a. $h$., HumboldtFruhstorfer, Ins.-Börse, v. 21, p. 389 (1904). Bai

- Taenaris a. h., id., Wien. Ent. Zeit., v. 24, p. $77,121,125$ (1905). - H. Stichel, Gen. Ins., fasc. 36, p. 51 (1906). - Frubstorfer: A. Seitz, Großschmett. Ende, v. 9, p. $414(1911) .-T$. a. $h$. forma principalis, H. Stichel, Tierreich, Lief. 34, p. 204 (part.) (1912).

? nigricans (Gr. Smith) Tenaris staudingeri ab. n., Grose Smith, Nov. Zool., v. 1, p. 584 (sine descr.) (1894).

? Drusilla selene var. myops (non $D$. $m$. Felder), Kirsch, Mitt. Zool. Mus. Dresden, v. 1 , p. 122 (1877).

- subsp. St a udingeri (Honr.) Tenaris s., Mittleres IIonrath, Berlin. Ent. Zeit., v. 33, p. 163, Nordostt. 2, f. 2 (1889). - Grose Smith, Nov. Neu-Guinea Zool., v. 1, p. 584 (1894). - Fürbringer (\& A. Pagenstecher): Semon, Zool. Forschungsreis. 5, Denkschr. Med.-Naturw. Ges. Jena, v. 8, p. (78) 238 (1895). - B. Hagen, Jahrb. Nassau. Ver. Naturk., v. 50, p. 80 (1897). - Rebel, Termesz. Füzet,, v. 21, p. 374 (1898). - Taenaris artemis 8., Fruhstorfer, Wien. Ent. Zeit., v. 24, p. 121, 125 (1905). - H. Stichel, Gen. Ins., fasc. 36, p. 52 (1906). - Tenaris s., Hauser, Verh. Zool,-Bot. Ges. Wien, v. 60, p. (38) (1910). - Taenaris artemis 8., Fruhstorfer: A. Seitz, Großschmett. Erde, v, 9, p. 415 (1911). T. a. s. forma principalis, $H$. Stichel, Tierreich, Lief. 34, p. 205 (1912). - T. 8. 8., W. Rothschild (\& Durrant), Rep. Brit. Ornithol. Un. Exped. Dutch N. Guinea, 2, Nr. 15, p. 19 (1915). - T. s., Röber, Nova Guinea, r. 13, Zool., livr. 1, p. 47 (1915). Eecke, 1. c., v. 15, Zool., p. 55 (1924). Tenaris s., N. D. Riley \& Gabriel, Cat. Type Specim. Rhopal. Brit. Mus., pars 1, p. 55 (1924).

forma nigricans (Hagen) Tenaris staudingeri var. n. (O. Staudinger MS), B. Hagen, Jahrb. Nassau. Ver. Naturk., v. 50, p. 80 (1897). - T. artemis 8. forma n., $\mathrm{H}$. Stichel, Gen. Ins., fasc. 36, p. 52 (1906). $T$. 8. forma nigrigans (error typogr., corr. p. 1111: nigricans), Fruhstorfer: A. Seitz, Großschmett. Erde, v. 9, p. 415 (1911 \& 27). - T. a. s. forma n., H. Stichel, Tierreich, Lief. 34, p. 206 (1912).

Typ.: KaiserWilhelmsland 
inf $u m a t a$ Fruhst. T. a. s. ab. i., Fruhstorfer, Wien. Ent. Zeit., v. 24, p. 78, 122 (1905).

forma artemides Fruhst. T. artemis s. Typ.: desgl.

àb, ar̃temides, Truhstorfer, Wien. Ent. Zeit., v. 24 , p. $79(1905)$. - T. artemis s. forma artemides, H. Stichel, Gen. Ins., fasc. 36, p. 52 (1906). - Fruhstorfer: A. Seitz, Großschmett. Erde, v. 9, p. 415 (1911). - H. Stichel, Tierreich, Lief. 34, p. 206 (1912).

forma eos Heller T. s, ab. e., K. M. Heller, Ent. Nachr., v. 20, p. 375 (1894). - T. artemis 8. ab. e., Fruhstorfer, Wien. Ent. Zeit., v. 24, p. 78, 122 (1905). - T. a. s. forma e., H. Stichel, Gen. Ins., fasc. 36, p. 52 (1906). - Fruhstorfer: A. Seitz, Großschmett. Erde, v. 9, p. 415 (1911). - H. Stichel, Tierreich, Liof. 34, p. 206 (1912).

forme lim bata Fruhst. T. a. s. ab. limbatus, Typ.: desgl. Fruhstorfer, Wien. Ent. Zeit., v. 24, p. 78, 122 (1905). - T. a. s. forma limbata, $H$. Stichel, Gen. Ins., fasc. 36, p. 52 (1906). Fruhstorfer: A. Seitz, Großschmett. Ende, v. 9. p. 415 (1911). - H. Stichel, Tierreich, Lief. 34, p. 205 (1912).

forma albicans (Hagen) Tenaris s. var. Typ.: Kaisera., B. Hagen, Jahrb. Nassau. Ver. Naturk., Wilhelmsv. 50, p. 80 (1897). - Taenaris artemis s. land ab. albicans, Fruhstorfer, Wien. Ent. Zeit., v. 24, p. $78,122(1905)$. - T. artemis s. forma albicans, H. Stichel, Gen. Ins., fasc. 36, p. 52 (1906). - Fruhstorfer: A. Seitz, Großschmett. Erdo, v. 9, p. 415 (1911). H. Stichel, Tierreich, Lief. 34, p. 206 (1912).

forma simonetta Fruhst. T. staudingeri ab. simonetta, Fruhstorfer, Wien. Ent. Zeit., v. 24, p. $80,122(1905)$ - - T. artemis st. forma si., Fruhstorfer: A. Seitz, Großschmett. Erde, v. 9, p. 415 (1911). - F. Stichel, . Tierreich, Lief. 34, p. 206 (1912).

T. a. s. forma. $b$ a $r$ b a $a$ (non Tenaris $b$. Kirby), H. Stichel, Gen. Ins., fasc. 36, p. 52 (part.) (1906).

forma $d r$ u e $n$ tia Fruhst. $T$. a. s. forma d., Typ.: desgl. Fruhstorfer: A. Seitz, Großschmett. Erde, v. 9, p. 415 , t. $101 \mathrm{~d}$ (1911). - H. Stichel, Tierreich, Lief. 34, p. 207 (1912).

forma $m \circ n \circ p s$ Fruhst. $T$. a. s. ab. m., Typ.:desgl. Fruhstorfer, Wien. Ent. Zeit., v. 24, p. 79, 122 (1905). - T. a. 8. forma m., H. Stichel, Gen. Ins., fasc. 36 , p. $52(1906)$. - T. a. 8 . ab. m., Fruhstorfer: A. Seitz, Großschmett. Erde, v. 9, p. 415 (1911). - T. a. 8. forma $m .$, H. Stichel, Tierreich, Lief. 34, p. 207 (1912). - T. s. ㅇ, ,form" m., Eecke, Nov. Guinea, 'v'. 15, Zool., p. 38 (1924). (Conf'. $T$. artemis in spec.).

forma lacrimans Fruhst. T. artemis stau- Typ.:desgl. dingeri (l.), Fruhstorfer: A. Seitz, Groß- 
schmett. Erde, v. 9, p. 415 (1911). - T. a. staudingeri forma $l .$, H. Stichel, Tierreich, Lief. 34, p. 205 (1912). (Conf. T. artemis in spec.).

forma op u le nta Stich. T. a. s. forma o., Typ.:

H. Stichel, Gen. Ins., fase. 36, p. 52 (1906). - T. a. s. (o.), Fruhstorfer: A. Seitz, Großschmett. Erde, v. 9, p. 415 (1911). H. Stichel, Tierreich, Lief. 34, p. 207, f. 39 (1912). (Conf. T. artemis in spec.).

forma ge m m a t a Fruhst. T. a. s. forma g., Typ.: ? H. Stichel, Tierreich, Lief. 34, p. 207 (1912). (Conf. T. artemis in spec.).

forma sidus Fruhst. Tr. a. staudingeri forma Typ.: ? sidus, H. Stichel, Tierreich, Lief. 34, p. 207 (1912). - T. a. s. (s.), Fruhstorfer: A. Seitz, Großschmett. Erde, v. 9, p. 415 (1911). (Conf. T. artemis in spec.).

subsp. J $a m e s i$ (Butl.) Tenaris j., A. G. Yule-Insel Butler, Proc. Zool. Soc. London, p. 766, t. 77 , f. 4 (ㅇ) (1876). - W. F. Kirby, Cat. Diurn. Lep., Suppl., App., p. 846 (1877). O. Staudinger (\& Schatz), Exot. Schmett., v. 1, p. 193, 198 (1887). - Tryon: J. P. Thomson, Brit. N. Guinea, App. 3, p. 257 (1892). - Taenaris artemis $j .$, Fruhstorfer, Wien. Ent. Zeit., v. 9, p. 77, 121 (1905). H. Stichel, Gen. Ins., fasc. 36, p. 52 (1906). - Fruhstorfer: A. Seitz, Großschmett. Erde, v. 9, p. 415 (1911). - H. Stichel, Tierreich, Lief. 34, p. 207 (1912). - W. Rothschild, Nov. Zool., v. 23, p. 304 (1916). - Tenaris $j .$, N. D. Riley \& Gabriel, Cat. Type Specim. Rhop. Brit. Mus., pars 1, p. 28 (1924).

? Tenaris artemis, Tryon: J. P. Thomson, Brit. N. Guinea, App. 3, p. 258 (1892). subsp. Sticheli (Fruhst.) Tenaris 8., Fruhstorfer, Ins.-Börse, v. 18, p. 373 (1901).-
Id., Berlin. Ent. Zeit., v. 47 , Sitzgs.-Ber., p. (20) (1902). - Id., Deutsch. Ent. Zeit. Lep., v. 14, 1901, p. 330 (1902). - Taenaris s., T. artemis s., Fruhstorfer, Wien. Ent. Zeit., v. 24, t. 1 ; p. 121, 125 (1905). T. a. s., H. Stichel, Gen. Ins., fase. 36, p. 52 (1906). - Fruhstorfer: A. Seitz, Großschmett. Erde, v. 9, p. 415 (1911). - H. Stichel, Tierreich, Lief. 34, p. 208 (1912).

? Drusilla urania (non Pap. u. Linné), Tryon, 2. Ann. Rep. Admin. Brit. N. Guinea, App. 5, sep., p. 7 (1890). - Id.: J. P. Thomson, Brit. N. Guinea, App. 3, p. 251 (1892).

subsp. electra (Fruhst.) Tenaris a. e., Fergusson

Fruhstorfer, Ins.-Börse, v. 21, p. 389 (1904). - Taenaris e., T. artemis e., id., Wien. Ent. Zeit., v. 24, p. 75, 121, t. 1 (1905). T. artemis affinis forma e., H. Stichel, Gen. Ins., fasc. 36, p. 53 (1906). - T. artemis e.,

Britisch-

Neu-Guinea (Milne Bai, ? Fly-River) 
Fruhstorfer: A. Seitz, Großschmett. Erde, v. 9 , p. 416 (1911). - T. a. barbata forma e., H. Stichel, Tierreich, Lief. 34, p. 209 (1912). subsp. barbata (Kirby) Tenaris b., W. Louisiaden F. Kirby, Ann. Mag. Nat. Hist., ser. 6, v. (Rossel3, p. 161 (1889). - Grose Sinith \& W. F. Insel) Kirby, Rhopal. Exot., v. 2, t. Tenaris 1, f. 1, 2 ; p. 1 (1894). - T. artemis $b$., Fruhstorfer, Ins.-Börse, v. 2i, p. 389 (1904). Taenaris artemis affinis ab. b., id., Wien. Ent. Zeit., v. 24, p. 122 (1905). - T. artemis staudingeri forma $b$., H. Stichel, Gen. Ins., fasc. 36, p. 52 (cit. part.) (1906). - T. artemis b., Fruhstorfer: A. Seitz, GroBschmett. Erde, v. 9, p. 416 (1911). - T. a. $b$. forma principalis, H. Stichel, Tierreich, Lief. 34, p. 208 (cit. part.) (1912). - Tenaris b., N. D. Riley \& Gabriel, Cat. Type Specim. Rhop. Brit. Mus., pars 1, p. 7 (1924).

forma affinis (Kirby) Tenaris a., W. F. RosselKirby, Ann. Mag. Nat. Hist., ser. 6, v. 3, p. Insel 162 (1889). - Grose Smith \& W. F. Kirby, Rhopal. Exot., v. 2, t. Tenaris 1, f. 3, 4; p. 2 (1894). - Taenaris art. aff., Fruhstorfer, Wien. Ent. Zoit., v. 24, p. 122 (1905). H. Stichel, Gen. Ins., fasc. 36, p. 53 (cit. part.) (1906). - Fruhstorfer: A. Seitz, GroBschmett. Erde, v. 9, p. 416 (1911). T. artemis barbata forma affinis, H. Stichel, Tierreich, Lief. 34, p. 208 (1912). - Tenaris affinis, N. D. Riley \& Gabriel, Cat. Type Specim. Rhop. Brit. Mus., pars 1, p. 5 (1924).

subsp. tine utus Fruhst. T. a. t., Fruh- Woodlarkstorfer, Wien. Ent. Zeit., v. 24, p. 80, p. Inseln 122 (1905). - Id.: A. Seitz, Großschmett. Erde, v. 9, p. 416 (1911). - W. Rothschild, Nov. Zool., v. 23, p. 305 (1916). - Tenaris t. (artemis.ssp.), N. D. Riley \& Gabriel, Cat. Type Specim. Rhop. Brit. Mus,, pars 1, p. 59 (1924).

T. artemis affinis (non Ten. affinis Kirby) (part.), H. Stichel, Gen. Ins., fasc. 36, p. 53 (1906).

T. $a$. barbata (non Ten, b. Kirby) (part.), II. Stichel, Tierreich, Lief. 34, p. 208 (1912). subsp. melanops (Grose Sm.) Tenaris m., SüdostGrose Smith, Ann. Mag. Nat. Hist., ser. 6, Neu-Guinea v. 19, p. 177 (Q) (1897). - Grose Smith (\& W. F. Kirby), Rhop. Exot., v. 3, t. Tenaris 5, f. 3 , 4; p. 18 (1898). - Taenaris artemis m., Fruhstorfer, Wien. Ent. Zeit., v. 24, p. $122(1905)$. - H. Stichel, Gen. Ins., fasc. 36 , p. 53 (1906). - Fruhstorfer: A. Seitz, Großschmett. Erde, v. 9, p. 416 (1911). - H. Stichel, Tierreich, Lief. 34, p. 209 (1912). 
subsp. nova Taenaris jamesi (non Butler), DarnleyG. A. Waterhouse \& Lyell, Butterfl. Austral., Insel p. 49 , t. 38 , f. 790 (1914).

(Papua

subsp. $q u$ uens landica Rothsch. T. a. q., Nord-

W. Rothschild, Nov. Zool., v. 23, p. 304 Queensland (1916).

Meeki Rothsch. T. m., W. Rothschild, Nov. Zool., v. Südwest23 , p. 305 , t. 3 , f. $3\left(\sigma^{*}\right)$, f. 7 .(口) $(1916)$. Holländisch-

Neu-Guinea

(Snow-

Gebirge,

Ober.

Setekwa-,

Oetakwa-

Fluß)

\section{Cohors g. Bioculafiformes}

H. Stichel, Gen. Ins., fasc. 36, p. 53 (1906). - Id., Tierreich, Lief. 34 , p. 209 (1912).

gorgo (Kirsch) Drusilla g., Kirsch, Mitt. Zool. Nördliches Mus. Dresden, v. 1, p. 121 (1877). - Tae- u. östlich. naris g., Fruhstorfer, Wien. Ent. Zeit., v. Neu-Guinea, 24, p. 125, 126 (1905). - 'H. Stichel, Waigeu Gen. Ins., fasc. 36, p. 53 (1906). - Eruhstorfer: A. Seitz, Großschmett. Erde, v. 9, p. 412 (1911). - H. Stichel, Tierreich, Lief. 34, p. 211 (1912).

subsp. gorgo (Kirsch) Drusilla g. (Tenaris Nordwestcatops var.), Kirsch, ut antea (1877). - HolländischTenaris g., O. Staudinger (\& Schatz), Exot. Neu-Guinea Schmett., v. 1, p. 192, 197 (part.) (1887). - (Mum, Taenaris g. g., Fruhstorfer, Wien. Ent. Zeit., Sorrong) v. 24, p. 87, 88, 122, 125 (1905). - H. Stichel, Gen. Ins., fasc. 36, p. 53 (1906). T. g., Fruhstorfer: A. Seitz, Großschmett. Erde, v. 9, p. 412 (1911). - T. g. g. forma principalis, H. Stichel, Tierreich, Lief. 34, p. 211 (cit. part.), f. 40 (ㅇ) (1912). T. g., Röber, Nova Guinea, v. 13, Zool., livr. 1, p. 47 (1915). - Eecke, l. c., p. 67 (1915).

subsp. mera Fruhst. T. g. m., Fruhstorfer, HolländischWien. Ent. Zeit., v. '24, p. 88, 89, 122, 125 (1905). - T. g. g. forma o m., H. Stichel, Gen. Ins., fasc. 36, p. 53 (1906). - T. $g$. m., Fruhstorfor: A. Seitz, Großschmett. Erde, v. 9, p. 412 (1911). - T. g. g. forma m., H. Stichel, Tierreich, Lief. 34, p. 212 (1912). subsp. arfakia Strand T. g. a. (T. gorgo gorgophone o forma?), Strand, Soc. Entom., v. 33, p. $12^{*}(1917)$ - Id., Bull. Soc. Zool. France, v. 51 p. 397 (1926).

subsp. danalis (Fruhst.) Tenaris gorgo d., Waigeu Fruhstorfer, Soc. Entom., v. 19, p. 129 (1904). - Taenaris g. d., id., Wien. Ent. Zeit., v. 24, p. $88,89,122$, t. 2 (1905). -

\section{Neu-Guinea, (Kajumera- Bai)}

Arfak-

Gebirge 
H. Stichel, Gen. Ins., fasc. 36, p. 53 (1906). - Fruhstorfer: A. Seitz, Großschmett. Erde, v. 9, p. 413 (1911). - H. Stichel, Tierreich, Lief. 34 , p. 212 (1912).

Tenaris gorgo (part.), O. Staudinger (\& Schatz), Exot. Schmett., v. 1, p. 192, 197 (1887).

subsp. gorgophone (Fruhst.) Tenaris gorgo gorgophone, Fruhstorfer, Soc. Entom., v. 19, p. 129 (1904). - Taenaris g. g., id., Wien. Ent. Zeit., v. 24, p. 87, 88, 122, 125 (1905). - H. Stichel, Gen. Ins., fasc. 36, p. 53 (1906). - Fruhstorfer: A. Seitz, Großschmett. Ende, v. 9, p. 413, t. $104 \mathrm{~d}$ (1911). - T. gorgo gorgophone forma principalis, H. Stichel, Tierreich, Lief. 34, p. 212 (1912). - T. gorgo gorgophone, Strand, Soc. Entom., v. 33, p. 12 (1918).

Tenaris gorgo, Heller, Ent. Nachr., v. 20 , p. 371 (1894). - Grose Smitb, Nov. Zool., v. 1, p. 359 (1894). - Grose Smith \& W. F. Kirby, Rhopal. Exot., v. 2, t. Tenaris 3 , f. 1,2 ; p. 10 (1895). - B. Hagen, Jahrb. Nassau. Ver. Naturk., v. 50, p. 81 (Biol.) (1897). - Rebel, Termesz. Fïzzet., v. 21, p. 374 , t. 18 , f. 12 (ㅇ) (1898).

forma $s i d u s$ (Stich.) Fruhst. T. gorgo gorgophone 8., Fruhstorfer: A. Seitz, Großschmett. Erde, v. 9, p. 413 (1911). - T. g. g. forma 8., H. Stichel, Tierreich, Lief. 34, p. 213 (1912).

forma simplex Fruhst. $T$. gorgo gorgophone desgl.

forma 8., Fruhstorfer: A. Seitz, Großschmett. Erde, v. 9, p. 413 (1911). - H. Stichol, Tierreich, Lief. 34, p. 213 (1912).

microps (Gr. Smith) Tenaris m., Groso Smith, Nov. NordostZool., v. 1, p. 358 (1894). - Grose Smith \& W. F. Kirby, Rhopal. Exot., v. 2, t. Tenaris 2 , f. 4 , 5 ; p. 6 (1894). - Taenaris m., Fruhstorfer, Wien. Ent. Zeit., v. 24, p. 122, 125 (1905). - H. Stichel, Gren. Ins., fasc. 36, p. 53 (1906). - Fruhstorfer: $\Lambda$. Seitz, Großschmett. Erde, v. 9, p. 213 (T. gorgo „A bweichung"?) (1911). - H. Stichel, Tierreich, Lief. 34, p. 213 (1912). - W. Rothschild, Nov. Zool., v. 23, p. 303 (1916).

- dimona (Hew.) Drusilla d., Hewitson, Exot. Butterfl., v. 4 (3), t. [2] Drusilla \& Hyantis Zeit. Lep., v. 2, p. 242 (1889). - Taenaris d., Fruhstorfer, Wien. Ent. Zeit., v. 24, p. 125, 126 (1905). - H. Stichel, Gen. Ins., fasc. 36, p. 53 (1906). - Fruhstorfer: A. Seitz, Großschmett. Erde, v. 9, p. 411 (1911). - H. Stichel, Tierreich, Lief. 34, p. 213 (1912).

subsp. dimona (Hew.) Drusilla d., He-, Aruwitson, ut antoa, f. 3, 4 (1862). - Tenaris Inseln 
d., Herrich-Schäffer, Corr.-Bl. Zool.-Min. Ver. Regensb., v. 19, p. 89 (1865). - W. F. Kirby, Cat. Diurn. Lep., p. 118 (1871). Ribbe, Corr.-Bl. Ent. Ver. Iris, v. 1, p. 83 (1886). - O. Staudinger (\& Schatz), Exot. Schmett., r. 1, p. 193, 201 (part.) (1887). - Ribbe, Deutsch. Ent. Zeit. Lep., v. 2, p. 242 (part.) (1889). - Grose Smith \& W. F. Kirby, Rhopal. Exot., v. 2, text. ad t. Tenaris 2, p. 6 (nota) (1894). - Taenaris d. d., Fruhstorfer, Wien. Ent. Zeit., v. 24, p. 82, 123 (1905). - H. Stichel, Gen. Ins., fasc. 36 , p. 54 (1906). - Fruhstorfer: A. Seitz, GroBschmett. Erde, v. 9, p. 54 (1911). - Tenaris a., A. Pagenstecher, Abl. Senckenb. Naturf. Ges., v. 33, p. 411 (1911). Strand, Soc. Int., v. 33, p. 12 (1918). Taenaris $d . d$. , H. Stichel, Tierreich, Lief. 34, p. 215 (1912). - Drusilla d., N. D. Riley \& Gabriel, Cat. Type Specim. Rhop. Brit. Mus., pars 1, p. 17 (1924).

- subsp. desdemona (Stauding.) Tenaris di-Ceram mona var. desdemona, $\mathrm{O}$. Staudinger (\& Schatz), Exot. Schmett., v. 1, p. 193, 201 (part.) (1887). - T. dimona, $T$. desdemona, T. dimona ab. desdemona, Ribbe, Deutsch. Ent. Zeit. Lep., v. 2, p. 201, 242 (1889). T. dimona (et ab. desdemona), id., Tijdschr. Ent., v. 34, p. 309 (1891). - Taenaris dimona desdemona, Fruhstorfer, Wien. Ent. Zeit., v. 24, p. 82, 123 (1905). - H. Stichel, Gen. Ins., v. 36, p. 54 (1906). Fruhstorfer: A. Seitz, Großschmett. Erde, v. 9, p. 412 (1911). - H. Stichel, Tierreich, Lief. 34, p. 215 (1912). - W. Rothschild, Nov. Zool., v. 22, p. 125 (1915).

subsp. of fak a Fruhst. Taenaris d. o., Fruh- Waigeu, storfer, Wien. Ent. Zeit., v. 24, p. 82, 83, Misol 126 (1905). - H. Stichel, Gen. Ins., fasc. 36, p. 54 (1906). - Fruhstorfer: A. Seitz, Großschmett. Erde, v. 9, p. 412 (1911). H. Stichel, Tierreich, Lief. 34, p. 216 (1912). W. Rothschild, Nov. Zool., v. 22, p. 125 (1915).

Tenaris dimona (part.), 0 . Staudinger (\& Schatz), Exot. Schmett., v. 1, p. 193, 201 (1887).

subsp. dimonata Stich. T. dimona dimo- Salwati nata, H. Stichel, Gen. Ins., fasc. 36, p. 54 (1906). - Fruhstorfer: A. Seitz, Großschmett. Erde, v. 9, p. 412 (1911). - H. Stichel, Tierreich, Lief. 34, p. 217 , f. 41 (1912).

subsp. sorronga Fruhst. T. d. s., Fruh- Holländischstorfer, Wien. Ent. Zeit., v. 24, p. 81, 83, Neu-Guinea 125 (1905). - H. Stichel, Gen. Ins., fasc. 36, p. 54 (1906). - Fruhstorfer: A. Seitz, Großschmett. Erde, v. 9, p. 412 (1911). -

(Sorrong, MamberanoFIuB) 
H. Stichel, Tierreich, Lief. 34, p. 216 (1912).

- Eecke, Nov. Guinea, v. 15, Zool., p. 37 (1924).

subsp. ka pa ura (Fruhst.) Tenaris d. k., Holländisch-

Fruhstorfer, Soc. Entom,, v. 19, p. 129 (1904). Neu-Guinea

- Id., Wien. Ent. Zeit., v. 24, p. 81, 83,

122, 125 (1905). - H. Stichel, Gen. Ins.,

fasc. 36, p. 54 (1906). - Fruhstorfer: A.

Seitz, Großschmett. Erde, v. 9, p. 412 (1911).

- H. Stichel, Tierreich, Lief. 34, p. 216

(1912). - W. Rothschild (\& Durrant), Rep.

Brit. Ornithol. Un. Exped. Dutch N. Guinea,

Nr. 15, p. 18 (1915). - Röber, Nora Guinea, v. 13, Zool., livr. 1, p. 47 (1915). Eecke, .l. c., p. 67 (1915).

subsp. A nma Fruhst. T. d. a., Fruhstorfer, ArfakDeutsch. Ent. Zeit. Iris, v. 29, p. 90, t. Gebirge 1 (0", 우) (1915).

subsp. zaitha Fruhst. T. d. z., Fruhstorfer, Südwest-

Ent. Rundschau, v, 31, p. 26 (1914). - Holländisch-

- ? T. d. "race" z., Eecke, Nova Guinea, v. Neu-Guinea

13, Zool., livr. 1, p. 67 (1915). - T. d. z,, (Eilanden-

Frubstorfer, Arch. Naturg., v. 81, 1915, FluB etc.)

Abt. A, Heft 11, p. 64 (1916).

? Drusilla dimona, Tryon, 2. Ann. Rep. Admin. Brit. N. Guinea, App. 5, sep., p. 7 (1890). - Id.: J. P. Thomson, Brit. N. Guinea, App., p. 258 (1892).

subsp. din ora (Gr. Smith \& W. F. Kirby) Nordost-

Tenaris d., Grose Smith \& W. F. Kirby,

Rhopal. Exot., v. 2, t. Tenaris 4, f. 3, 4 (q);

p. 14 (1896). - Taenaris dimona dinora,

Fruhstorfer,. Wien. Ent. Zeit., v. 24, p. 83, 122, 125 (1905). - H. Stichel, Gen. Ins., fasc, 36, p. 54 (1906). - Fruhstorfer: A. Seitz, Großsschmott. Erde, v. 9, p. 412 (1911). - T. dimona dinora forma principalis, $\mathrm{H}$. Stichel, Tierreich, Lief. 34, p. 217 (1912).

$D r u$ silla dimona, Kirsch, Mitt. Zool. Mus. Dresden, v. 1. II, p. 122 (1877). - Tenaris d. var., Grose.Smith, Nov. Zool., v. 1, p. 360 (1894).

Tenaris d. var. desdemona (part.), 0 . Staudinger. (\& Schatz), Exot. Schmett., v. 1 , p. 193, 201 (1887).

forma trita Stich. T. dimona dinora forma Typ.: t., H. Stichel, Gen. Ins., fase. 36, p. 54 (1906). - T. dimona dinora t., Fruhstorfer: A. Seitz, Großschmett. Erde, v. 9, p. 412 (normale Ozellenform) (1911). - T. dimona dinora forma $t$., H. Stichel, Tierreich, Lief. 34 , p. 218 (1912).

subsp. a reia (Fruhst.) Tenaris $d$. a., Fruh- Mittleres storfer, Soc. Entom., v. 19, p. 129 (1904). - NordostTaenaris d. a., id., Wien. Eint. Zeit., v. 24, Neu-Guinea p. $82,123,125$; T. a., t. 2 (1905). - T. (Astrolabe d. a., H. Stichel, Gron. Ins., fasc. 36, p. 54 Bai, (1906). - Fruhstorfer: A. Seitz, Großschmett. Ende, v. 9, p. 412, t. $104 \mathrm{~d}$ (O)

Neu-Guinea (HumboldtBai)

\section{Humboldt- \\ $\mathrm{Bai}$}

Stefansort

etc.) 
(1911). - Strand, Mitt. Zool. Mus. Berlin, v. 5, p. 470 (1911). - H. Stichel, Tierreich, Lief. 34, p. 218 (1912).

Tenaris dimona, $T$. d. ab. dinora (non Gr. Smith \& Kirby), B. Hagen, Jahrb. Nassau. Ver. Naturk., v. 50, p. 81 (1897). - T. bimona, Rebel, 'I'ermesz. Füzet., v. 21, p. 374 (1898).

subsp. tha em a Fruhst. T. d. t., Fruh- Südoststorfer, Deutsch. Ent. Zeit., Iris, v. 29, p. Neu-Guinea 91 (1915).

(Finschhafen)

subsp. s o $p h a$ in eta Fruhst. T. d. s., Fruh- Südweststorfer, Ent. Rundschau, v. 31, p. 27 (1914). Neu-Guinea

(Yule Insel)

dina (Stauding.) Tenaris d., O. Staudinger, Deutsch. Neu-Guinea Ent. Zeit. Lop., v. 7, p. 112 (1894).

subsp. din a (Stauding.) Tenaris d., O. Stau- Mittleres dinger, Deutsch. Ent. Zeit. Lep., v. 7, p. Nordost112 (o'); p. 357 (क) (1894). - Heller, Neu-Guinea Ent. Nachr., v. 20, p. 376 (1894). - Grose Smith \& W. F. Kirby, Rhop. Exat., v. 2, t. Tenaris 4, f. 1, 2(o"); p. 13 (1896). - B. (Kaiser-

Hagen, Jahrb. Nassau. Ver. Naturk., v. 50, Wilhelmsp. 81 (1897). - T a en aris d., Fruhstorfer, Wien. Ent. Zeit., v. 24, p. 123, 125 (1905). - H. Stichel, Gen. Ins., fasc. 36, p. 54 (1906). - Fruhstorfer: A. Seitz, Großschmett. Erde, v. 9, p. 412 (1911). H. Stichel, Tierreich, Lief. 34, p. 218 (1912). subsp. insularis Rothsch. T. $d_{\text {. }}$ i., W. Rothschild, Nov. Zool., v. 23 , p. 302, t. 3 , f. $1\left(\sigma^{7}\right), 2$ (q) (1916).

subsp. sordidior Rothsch. T. $d$. s., W. SüdwestRothschild, Nov. Zool., v. 23, p. 303 (1916). Holländisch

Neu-Guinea

(Snow Mountains etc.)

bioculata (Guér.-Mén.) Morpho bioculatus, Guérin- Waigeu, Méneville, Voy. Coquille, Atl. Ins., t. 17 (1830). - Taenaris b., Fruhstorfer, Wien. Ent. Zoit., v. 24 , p. 125 (1905). $-T$. bioculata, H, Stichel, Gen. Ins., fase. 36, p. 55 (1906). - T. bioculatus, Fruhstorfer: A. Seitz, Großschmett. Erde, v. 9, p. 413 (1911). - T. bioculata, H. Stichel, Tierreich, Lief. 34, p. 219 (1912).

subsp. bi с с и la ta (Guér.-Mén.) Morpho bi- Waigeu oculatus, Guérin-Méneville, ut antea, t. 17, f. 1 (1830). - Drusilla b., Hewitson, Exot. Butterfl., v. 4 (3), t. [2] Drusilla \& Hyantis, f. 1, 2 (1862). - Tenaris b., Herrich-Schäffer, Corr.-Bl. Zool.-Min. Ver. Regensb., v. 19, p. 89 (1865). - W. F. Kirby, Cat. Diurn. Iep., p. 118 (1871). - Drusilla 
bioculata, J. G. Ward, Ins. abroad, p. 592 (1874). - Tenaris bioculatus, O. Staudinger (\& Schatz), Exot. Schmett., v. 1, t. 64, p. 193, 201 (1886 \& 87) - H. Goss; Proc. Ent. Soc. tondon, p. XI (1888). K. Jordan, Nov. Zrool, v. 5, p. 390, t. 15, f. 63 (antenna) (1898). - Taenaris b. b., Fruhstorfer, Wien. Ent. Zeit., v. 24, p. 89, 123 (1905). - T. bioculata b., H. Stichel, Gen. Ins., fasc. 36 , p. 55 (1906). - $T$. bioculatus b., Frulastorfer: A. Seitz, Großschmett. Frde, v. 9, p. 413 (1911). - T. bioculata b., H. Stichel, Tierreich, Lief. 34, p. 220 (1912).

indra (Boisd.) Hyades i., Boisduval, Voy. Astrolabe, Faun. Ent., pars 1, p. 158 (1832). - Guérin-Méneville, Voy. Coquille, Zool., v. 2. II, p. 282 (post 1832).

$\rho$... subsp. pallida Fruhst. $T$. bioculatus $p$., HolländischFruhstorfer, Wien. Ent. Zeit., v. 24, p. 89, Neu-Guinea 123, 125 (1905). - T. bioculata p., H. (Sorrong, Stichel, Gen. Ins., fase. 36, p. 55 (1906). - Kajumera, $T$. bioculatus $p$., Fruhstorfer: A. Seitz, Groß- Mamberan), schmett. Erde, v. 9, p. 413, t. $101 \mathrm{~d}$ (q) (1911). - T. bioculata p., H. Stichel, Tierneich, Lief. 34, p. 221 (1912). - T. bioculatus p., Röber, Nova Guinea, v. 13, Zool., livr. 1, p. 47 (1915). - Eecke, 1. c., p. 67 (1915). - Id., 1. c., v. 15, Zool., p. 37 (1924).

subsp. grisescens Rothsch. T. bioculatus Holländischg., IV. Rothschild (\& Durrant), Rep. Brit. Neu-Guinea Ornithol. Exp. Dutch N. Guinea, 2, Nr. (Base Camp) 15 , p. 18 (1915).

subsp. avarea Fruhst. T. bioculata a., Holländisch Fruhstorfer, Arch. Naturg., v. 81, 1915, Neu-Guinea Abt. A, Heft 11, p. 65 (1916).

(? Südwest)

- subsp. charonides (Stauding.) Tenaris bi- Humboldtoculatus var. .c., O. Staudinger, Deutsch. BaiEnt. Zeit. Lep., v. 7, p. 110 (1894). - Re- Astrolabe bel, Termesz. Füzet., v. 21, p. 374 (1898). Bai, - Taenaris b. c., Fruhstorfer, Wien. Ent. Zeit., v. 24, p. 89, 123, 125 (1905). - $T$. bioculata c., H. Stichel, Gen. Ins., fasc. 36 , p. 55 (1906). - $T$. bioculatus c., Hauser, Verh. Zool.-Bot. Ges. WVien, v. 60, p. (38) (1910). - Fruhstorfer: A. Seitz, Großschmett. Frde, v. 9, p. 413 (1911). - Strand, Archiv f. Naturgesch., 1912, A. 11, p. 65.$T$. bioculata c., H. Stichel, Tierreich, Lief. 34, p. 221 (1912).

? Drusillabioculata, Kirsch, Mitt. Zool. Mus. Dresden, v. 1, p. 123 (1877). - D. bioculatus, Ch. Oberthür, Ann. Mus. Oiv. Stor. Natural. Genova, v. 15, p. 508 (1880). Tenaris b., O. Staudinger (\& Schatz), Exot. Schmett., v. 1, p. 193, 201 (1887). 
- subsp. charon (Stauding.) Tenaris bioculatus var. c., O. Staudinger (\& Schatz), Exot. Schmett., v. 1, p. 193, 201 (1887). - ? B. Hagen, Jahrb. Nassau. Ver. Naturk., v. 50, p. 81 (1897). - Taenaris 6 . c., Truhstorfer, Wien. Ent. Zeit., v. 24, p. 89, 123, 125 (1905). - T. bioculatus c., H. Stichel, Gen. Ins., fasc. 36, p. 55 (1906). - T. bioculatus c.; Fruhstorfer: A. Seitz, Großschmett. Ende, v. 9, p. 413 (1911). - T. bioculata c., H. Stichel, Tierreich, Lief. 34, p. 222 (1912).

subsp. charondas Fruhst. T. bioculatus c., Fruhstorfer: A. Seitz, Großschmett. Erde, v. 9, p. 413 (1911). - T. bioculata c., H Stichel, Tierreich, Tief. 34, p. 222 (1912).

? Drusilla bioculata, Tryon, 2. Ann. Rep. Admin. Brit. N. Guinea, App. 5, p. 7 (1890). - Tenaris b., id.: J. P. Thomson, Brit. N. Guinea, App. 3, p. 257 (1892).

subsp. camaronensis Rothsch. T. biocu- Britischlatus c., W. Rothschild, Nov. Zool., v. 23, p. 303 (1916).

Britisch

Südwest-

Neu-Guinea

(Port

Moresby) -

? Südost-

Neu-Guinea

(Finsch-

hafen,

Simbang)

Britisch-

Neu-Guinea

(Aroa-? Fly River)

Neu-Guinea

(Owen

Stanley

Range)

\section{Sectio Melainarini}

H. Stichel, Gen. Ins., fasc. 36, p. 55 (1906). - Id., Tierreich, Lief. 34, p. 222 (1912).

Morphotenaris Fruhstorfer et alii, vide p. 85 .

Schönbergi (Fruhst.) Mor photenaris s., Fruh- Neu-Guinea storfer, Ent. Nachr., v. 19, p. 317 (1893). Morphotaenaris s., Taenaris s., id., Wien. Ent. Zeit., v. 24, p. 122,125 (1905). - T. 8., H. Stichel, Gen. Ins., fasc. 36 , p. 55 (1906). Morphotenaris s., Fruhstorfer: A. Seitz, Großschmett. Erde, r. 9, p. 424 (1911). - Taenaris s., H. Stichel, Tierreich, Lief. 34, p. 222 (1912).

subsp. Schönbergi (Fruhst.) Morphotenaris s., Fruhstorfer, ut antea (1893). 'Tenaris s., O. Staudinger, Deutsch. Ent. Zeit. Lep., v. 6, 1893, p. 363 (1894). - Morphotenaris s., Fruhstorfer, Berlin. Ent. Zeit., v. 39, p. 248 , t. 17 , f. 3 (1894). - Id., Ent. Zeit. Stettin, r. 55, p. 127 (1894). Tenaris s., B. Hagen, Jahrb. Nassau. Ver. Naturk., v. 50, p. 81 (1897). - Morphotaenaris s. s., Fruhstorfer, Wien. Ent. Zeit., v. 24, p. 122 , Taenaris s., 1. c., p. $125(1905)$. - T. s. s., H. Stichel, Gen. Ins., fasc. 36, p. 55 (1906). - Morphotenaris s., E. Werner, Kaiser Wilhelmsland, p. 170 (1911). - Fruhstorfer: A. Seitz, Großschmett. Erde, v. 9, p. 424, t. $104 \mathrm{~d}$ (1911). - Taenaris s. s., H. Stichel, Tierreich, Lief. 34, p. 223 (1912).

Mittleres

Nordost-

Neu-Guinea

(Kaiser-

Wilhelms-

Land) 
- Morphotenaris s. 8., W. Rothschild \& Durrant, Rep. Brit. Ornithol. Un. Exped. Dutch N. Guinea, 2, Nr. 15, p. 20 (1915). subsp. littoralis (Rothsch.) Morphotenaris s. l., W. Rothschild (\& Durrant), Rep. Brit. Ornithol. Un. Exped. Dutch N. Guinea, 2, Nr. 15, p. 20 (nota) (1915). - Id., Nov. Zool., v. 23, p. 307, t. 3, f. 4 (1916).

subsp. nivescens (Rothsch.) Morphotenanaris n., W. Rothschild, Nov. Zool., v. 3, p. 92 (1896). - Grose Smith (\& W. F. Kirby), Rhopal. Exot., v. 3, t. Morphotenaris \& Hyantis, f. 1, p. 9 (1898). - Morphotaenaris schönbergi ${ }_{n}$., Taenaris s. n., Fruhstorfer, Wien. Ent. Zeit., v. 24, p. 122, 125 (1905). - T. s. n., H. Stichel, Gen. Ins., fasc. 36, p. 55 (1906). - Morphotenaris s. n., Fruhstorfer: A. Seitz, Großschmett. Erde, v. 9, p. 424 (1911). - Taenaris s. n., H. Stichel, Tierreich, Lief. 34, p. 224 (1912). subsp. Wollastoni (Rothsch.) Morphotenaris s. w., W. Rothschild (\& Durrant), Rep. 2, Nr. 15, p. 19 (1915). - Id., Nov. Zool., จ. 23, p. 307, t. 3 , f. 5 (1916).

subsp. Kenricki (Beth.-Baker) Morphotenaris k., Bethune-Baker, Ann. Mag. Nat. Hist., ser. 8, v. 4, p. $184 \cdot(1909)$. - Taenaris schönbergi $k$., H. Stichel, Tierreich, Lief. 34, p. 223 (1912). - Morphotenaris s. K., W. Rothschild (\& Durrant), Rep. Brit. Ornithol. Un. Exp. Dutch Guinea, 2, Nr. 15, p. 20 (1915). - Morphotaenaris k., Talbot, Proc. Ent. Soc. Iondon, 1920, p. LXXII (1921).

subsp. wandammensis (Joicey \& Talb.) NordostMorphotaenaris 8. w., Joicey \& Talbot, Ann. HolländischMag. Nat. Hist., ser. 8, v. 17, p. 73, t. 6, Neu-Guinea f. 5 (1916). - Eecke, Nov. Guinea, livr. 15, Zool., p. 39 (1924).

? Morphotaenaris schönbergi, Talbot, Proc. Ent. Soc. London, 1920, p. LXXI (1921).

Südost-

Neu-Guinea

(Collingwood-

Bai,

Huon Golf)

Britisch-

Neu-Guinea

(Eafa

District)

Süd-

Holländisch-

Neu-Guinea

(Utalrva-

Fluß,

Mt. Goliath)

Nordost-

Holländisch-

Neu-Guinea

(Arfak)

(Wan-

dammen-

Gebirge),

Mamberano-

Fluß)

subsp. weylandensis (Joicey \& Talb.) NordwestMorphotaenaris s. w., Joicey \& Talbot, Bull. HolländischHill Mus., v. 1, p. 328 (1922).

Neu-Guinea (WeylandGebirge)

subsp. Adamsi (Lathy) Morphotenaris a., HolländischLathy, Entomologist, จ. 42, p. 272 (1909). Neu-Guinea - N. D. Riley \& Gabriel, Cat. Type Specim. (Nina-Tal) Rhop. Brit. Mus., pars 1, p. 5 (1924). 


\section{Subfamilia Hyanfinae $\mathrm{H}$. Stich,}

Hy a $n$ tidac, Röbor, Soc. Entom., v. 19, p. 170 (1905). - H. Stichel, Berlin. Ent. Zeit., v. 49, 1904, p. 306 (1905). - Hyantinae, id., 1. c., v. 49, 1904, p. 306 (1905). - Id., Gen. Ins.; fasc. 39, p. 1 (1905). - "Hyantiden", A. Pagenstecher, Geogr. Verbr. Schmetterl., p. 413 (part.) (1909). - Hyantiidae, Röber, Nova Guinea, v. 13, Zool., livr. 1, p. 46 (1915). - Hyantinae, Eecke, 1. c., p. 66 (1915). - Id., 1. c., v. 15, Zool., p. 37 (1924).

Nym $p$ halidae (part.), W. Rothschild \& K. Jordan, Nov. Zool., v. 12, p. 454 (1905). - K. Jordan, Proc. Int. Soc. Iondon, v. 5. II, p. 58 (1930).

Brassolidae, Strand, Mitt. Zool. Mus. Berlin, v. 5, p. 470 (1911).

\section{Genus Hyantis Hew.}

Hewitson, Exot. Butterfl., v. 4 (3), t. [2] Drusilla \& Hyantis (1862). - Herrich-Schäffer, Corr.-Bl. Zool.-Min. Ver. Regensb., v. 19, p. 89 (1865). - W. F. Kirby, Cat. Diurn. Lep., p. 119 (1871). — S. H. Scudder, Proc. Amer. Ac. Sci., v. 10, p. 193 (1875). - Wallace, Googr. Distrib. Anim., v. 1, p. 404 (1876). - C. Crüger, (sec. Kirby), Berlin. Ent. Zeit., v. 25, p. 111 (1881). - L. Glaser, Cat. Etymol. Col. Lep., p. 294 (spec. omiss.) (1887). - O. Staudinger' (\& Schatz), Exot. Schmett., v, 1, p. 191 (1887). - Röber: O. Staudinger \& Schatz, Exot. Schmett., v. 2, t. 31; p. 184, 188 (Morphol.) (1888 \& 89). - E. Haase, Corr.-Bl. Ent. Ver. Iris, v. 1, p. 311 (1888). - B. Gerhard, Berlin. Ent. Zeit., v. 27, p. 181 (distr. geogr.) (1893). - K. M. Hcller, Entom. Nachr., v. 20, p. 369 (1894). Hyautis (error typogr.!), Grose Smith, Nov. Zool., v. 1, p. 357 (1894). - Ilyantis, K. Jorlan, Nov. Zool., v. 5, p. 390 (antenna) (1898). H. Stichel, Deutsch. Ent. Zeit. Iris, v. 15, p. 59 (1902). - Id., Berlin. Ent. Zeit., v. 49, 1904, p. 303 (1905). - Id., Gen. Ins, fasc. 39, p. 2, t. f. 1 (Marphol.) (1905). - A. Pagenstecher, Geogr. Verbr. Schmett., p. 413 (1909). - Fruhstorfer: A. Seitz, Großschmett. Erde, v. 9, p. 408 (1911). - Hyanthis, Eecke, Nova Guinea, v. 13, Zool., livr. 1, p. 67 (1915).

- hodeva Hew. H. h., Howitson, Exot. Butterfl., v. 4 Waigeu,

(3), t. [2] Drusilla \& Hyantis (1862). H. Stichel, Gen. Ins., fasc. 39, p. 4 (distrib. corrigend.) (1905). - Fruhstorfer: A. Seitz, Großschmett. Erde, v. 9, p. 408 (1911). subsp. hode va Hew. H. $h$., Hewitson, Exot. Butterfl., v. 4 (3), t. 2 Drusilla \& Hyantis, f. 5, 6 (1862). - Kirsch, Mitt. Zool. Mus. Dresden, v. 1, p. 123 (part.) (187i). O. Staudinger (\& Schatz), Exot. Schmett., v. 1 , t. 63 , p. $191(1886 \& 87)$. - K. M. Heller, Entom. Nachr., v. 20, p. 370 (variaNeu-Guinea tio) (1891). - O. Staudinger, Deutscl. Ent. Zeit. Lep., v. 7, p. 107 (1894). - Hyautis (!) h., Grose Smith, Nov. Zool., v. 1, p. 357 (1894). - Hyantis h., B. Hagen, Jahrb. Nassau. Ver. Naturk., v. 50, p. 79 (1897). H. Stichel, Berlin. Ent. Zeit., v. 49, 1904, p. 310, t. 4, f. 1 (forma principalis) (1905). Id., Gen. Ins., fasc. 39 , p. 4 (cit. part.),

Waigeu,

Nord-

HolländischNen-Guinea (Begrenzung unbestimmt) 
(distr. corrigend.) (1905), - Fruhstorfer: A. Seitz, Großschmett. Erde, v. 9, p. 408 (1911). - Röber, Nova Guinea, v. 13, Zool., livr. 1, p. 46 (1915). - Eecke, 1. c., p. 67 (1915). - N. D. Riley \& Gabriel, Cat. Type Specim. Rhop. Brit. Mus., pars 1, p. 25 (1924).

forma inf r m a $t$ a Stauding. $H$. $h$. var, $i ., 0$. Typ.:

Staudinger, Deutsch. Ent. Zeit. Lep., v. 7, p. 108 (1894). - H. i., $H$. hodeva var. $i .$, Grose Smith (\& W. F. Kirby), Rhopal. Exot., v. 3, t. Morphotenaris, Hyantis, $\mathbf{f}$. 2 ; p. 2 (1898). - H. h. var. i., Röber, Ent. Zeit. Stettin, v. 64 , p. 342 (1903). - H. $h$. forma i., H. Stichel, Berlin. Ent. Zeit., 49,1904, p. 312 , t. 4 , f. 8 (1905). - Id., Gen. Ins., fasc. 39 , p. 4 (1905). $-H$. h. $i$., Fruhstorfer: A. Seitz, Großsclimett. Ende, v. 9, p. 408 (Ábänderung) (1911).

subsp. fuliginosa Gr. Smith $H$. f., $H$. Nordwesthodeva var. $f$., Grose Smith (\& W. F. HolländischKirby), Rhopal. Exot., v. 3, t. Morpho- Neu-Guinea tenaris, Hyantis, f. 3 ; p. 2 (1898). $-H$. h. (Kapaur, forma f., H. Stichel, Berlin. Ent. Zeit v. 49, 1904, p. 312 (1905). - Id., Gen. Ins., fasc. 39, p. 4 (1905). - H. $\hbar$. f., Fruhstorfer: A. Seitz, Großschmett. Erde, v. 9, p. 408 (1911). - Röber, Nova Guinea, v. 13, Zool., livr. 1, p. 46 (1915). - H. h. ,race" f., Eecke, 1. c., p. 67 (1915). - H. h. f., W. Rothschild, Nov. Zool., v. 16, p. 300 (1916). - Eecke, Nova Guinea, v. 15, Zool., p. 37 (1924).

forma melañomata (Stauding.) $H . h$. $f$. Holländischforma $m$., Eecke, Nova Guinea, v. 15, Zool., Neu-Guinea p. 37 (1921) (Forma fucosa!)

forma microphthalma (Heller) $H$. $h$. desgl.

$m$, Röber, Nova Guinea, v. 13, Zool., livr.

1 , p. 46 (1915). - II. h. ,form" m., Eecke,

1. c., p. 67 (1924). (Forma fucosa!).

forma ox yophth alma Stich. H. $h$. forma Typ.: Andai

o., H. Stichel, Gen. Ins,, fasc. 39, p. 4, t.,

f. 3 (1905). - H. h. o., Fruhstorfer: A.

Seitz, Großschmett. Erde, v. 9, p. 408 (Nebenform) (1911). - H. h. o., Lecke, Nova Guinea, v. 13, Zool., livr. 1, p. 67, t. 3, f. 7 (1915). - H. h. fuliginosa ab. o., W. Rothschild, Nov. Zool., v. 23, p. 300 (1916). subsp. e marg i nat a Fruhst. $H . h . e .$, Fruh- Holländischstorfer, Arch. Naturg., v. 81, Abt. A., Heft Neu-Guinea 11 , p. 64 (1921)

(? Südwest)

subsp. a lb ip la ga Rothsch. H. a., W. Roth- Holländischschild (\& Durrant), Rep. Brit. Ornithol. Neu-Guinea Un. Exped. Dutch N. Guinea, 2, Nr. 15, (Utakvap. 18 (1915). - H. hodeva subsp. a., id., Fluß) Nov. Zool., v. 23, p. 300 (1916).

forma pallida Rothsch. H. h. a. ab. p., Typ.: SnowW. Rothschild, Nov. Zool., v. 23, 300 (1916). Mountains 
subsp. $x$ a $n$th o phtha $l m a$ Röb. H. $x$., Rö- Südost-

ber, Ent. Zeit. Stettin, v. 64, p. 339 (1903). Neu-Guinea

- $H$. hodeva forma $x ., H$. Stichel, Berlin. Ent. Zeit., v. 49, 1904, p. 310, t. 4, f. 3 (1905). - Id., Gen. Ins., fasc. 39, p. 4 (1905). - H. h. x., Fruhstorfer: A. Seitz, Großschmett. Ende, v. 9, p. 408 (1911). subsp. $m$ c la nom a ta Stauding. $H . h$. var. Ehemalig. m., o. Staudinger, Deutsch. Ent. Zeit., Lep., จ. 7, p. 109 (1894). - Heller, Entom. Nachr. v. 20, p. 369 (1894). - B. Hagen, Jahrb. Nassau. Ver. Naturk., v. 50, p. 79 (1897). - H. m., Röber, Fnt. Zeit. Stettin, v. 64, p. 342 (1903). - H. hodeva forma m., H. Stichel, Berlin. Ent. Zeit., v. 49, 1904, p. 311 , 312, fig. A (O), t. 4, f. 7 ( ( ) (1905). - Id., Gen. Ins., fasc. 39, p. 4, t., fig. 2 (Q) (1905). - H. h. m., Fruhstorfer: A. Seitz, Großschmett. Erde, จ. 9, p. 408, t. $104 \mathrm{c}\left(\varphi, \sigma^{\top}\right)$ (1911).

forma Hagen $i$ Röb. $H$. h., Röber, Ent. Zeit. Stettin, v. 64, p. 341 (1903). - $H$. (hodeva) hageni, Fruhstorfer: A. Seitz, Großschmett. Erde, v. 9, p. 408 (melanomata, Hauptform) (1911).

H. hodeva (non Hewitson), H. Stichel, Gen. Ins., fasc. 39,1904 , p. 4 (in cit.) (1905). forma helvola Stich. $H$. hodeva forma helvola, H. Stichel, Berlin. Ent. Zeit., v. 49, 1904, p. 311 (1905). - Id., Gen. Inख,', fasc. 39 , p. 4 (1905). - H. (hodeva) helvola, Fruhstorfer: A. Seitz, Großschmett. Ende, จ. 9, p. 408 (Nebenform) (1911).

forma $m$ ic rop $h t h a l m a$ Heller $H . h . m$., Heller, Entom. Nachr., v. 20, p. 371 (1894). - H. h. var. m., Röber, Ent. Zeit. Stettin, v. 64, p. $342(1903)$. - H. $h$. forma $m .$, . Stichel, Berlin. Ent. Zeit., v. 49, 1904, p. 311 , t. 4, f. 5 (1905). - Id., Gen. Ins., fasc. 39, p. 4 (1905). - H. h. m., Fruhstorfer: A. Seitz, Großschmett. Erde, v. 9, p. 408, t. $104 \mathrm{c}(1911)$.

forma anulata Stich. $H$. $h$. forma a., H. Typ.: Stichel, Berlin. Ent. Zeit., v. 49, 1904, p. Neu-Guinea 311 , t. 4, f. 6 (1905). - Id., Gen. Ins., fasc. 39, p. 4 (1905). - H. h. a., Fruhstorfer: A. Seitz, Großschmett. Erde, v. 9, p. 1 408 (Aberration) (1911).

\section{Genus Bigaena Eecke}

Eecke, Nov. Guinea, v. 13, Zool., livr. 1, p. 66, fig. 2 (Morphol.) (1915).

pumilio Eecke B. p., Eecke, Nova Guinea, v. 13, NordwestZool., livr. 1, p. 66, t. 2, f. 9 (1915). Holländisch-

Éhemalig.

Deutsch-

Neu-Guinea

Doutsch-

Typ.:

Neu-Guinea

$$
\text { Grinea }
$$

(eu-Guinea 


\section{Genus Morphopsis Ch. Oberth.}

Ch. Oberthür, Ann. Mus. Oiv. Stor. Natural. Genova, v. 15, p. 513 (1880). - B. Hagen, Jahrb. Nassau. Ver. Naturk., v. 50, p. 79 (1897). - H. Stichel, Berlin. Ent. Zeit., v. 49, 104, p. 303 (1905). - Id., Gen. Ins., fase. 39, p. 4, t., fig. 4 (Morphol.) (1905): A. Pagenstecher, Geogr. Verbr. Schmett., p. 413 (1909). - Fruhstorfer: 1. Seitz, Großschmett. Erde, v. 9, p. 408 (1911). Eecke, Nova Guinea, v. 13, Zool., livr. 1, p. 67 (1915). - W. Rothschild, Nov. Zool., v. 23, p. 300 (1916). - Talbot, Proc. Ent. Soc. London, 1920, p. LXXII (1921).

- Albertisi Oberth. M. a., Ch. Oberthür, Ann. Mus. Neu-Guinea, Civ. Stor. Natural. Genova, v. 15, p. 513 Waigeu (1880). - H. Stichel, Gen. Ins., v. 39, p. 6 (1905). - Fruhstorfer: A. Seitz, Großschmett. Erde, v. 9, p. 409 (1911). - W. Rothschild, Nov. Zool., v. 23, p. 300, 302 (1916).

subsp. Albertis $i$ Oberth. $M$. a., $M$. d'Al- Nordostbertisi, Ch. Oberthür, Ann. MLus. Civ. Stor. Neu-Guinea Natural. Genova, v. 15, p. 513 , t. 2 , f. 3 (1880). - M. a., Grose Smith, Nov. Zool., v. 1 , p. 357 (purt.: Q) (1894). - H. Stichel, Berlin. Ent. Zeit., v. 49, 1904, p. 307 (1905). - M. a. a., H. Stichel, Gen. Ins., fasc. 39, p. 6 (1905). - Fruhstorfer: A. Seitz, Großschmett. Finde, v. 9, p. 409 (part.) (1911). M. a., Strand, Mitt. Zool. Mus. Berlin, v. 5, p. 470 (1911). - Röber, Nova Guinea, v. 13, Zool., livr. 1, p. 46 (1915). - Eecke, l. c., p. 67 (1915), - M. a: a., W. Rothschild, Nov. Zool., v. 23, p. 300, 302 (1916).

subsp. $k$ u mus $i$ i Rothsch. M.a.k., W. Roth- Nordostochild, Nov. Zool., v. 23, p. 301, 302 (1916).

subsp. astrolabensis Stich. M. albertisi astrolabensis, H. Stichel, Berlin. Ent. Zeit., v. 49, 1904, p. 307 (1905). - Id., Gren. Ins., fasc. 39 , p. 6; t., fig. 5 (1905). $-M$. albertisi astrolabiensis (!), Fruhstorfer: A. Seitz, Großschmett. Erde, v. 9, p. 409, t. $104 \mathrm{c}$ (GeelwinkBai: Andai, HumboldtBai) (1911). - W. Rothschild, Nov. Zool., v. 23, p. 301,302 , t. 4 , f. 1 (1916).

M. d'A ibertisi, B. Hagen, Jahrb. Nassau. Vor. Naturk., v. 50 , p. 79 (1897).

subsp. mambarensis Rothsch. M. a. m., NordIV. Rothschild, Nov. Zool., v. 23, p. 301, 302 Neu-Guinea (1916).

Neu-Guinea

(Kumusi-

Fluß)

Ehemalig.

Deutsch-

Neu-Guinea

(Astrolabe-

Bai)

(Mambare-

Fluß)

subsp. Miln e $i$ Rothsch. M. a. m., W. Roth- Britischschild, Nov. Zool., v. 23, p. 301, 302, t. 4, f. 2 Südost(1916).

Neu-Guinea

(Milne Bai)

subsp. 8 e ta $f w$ w $e n$ s $i$ s Rothsch. M. a. s., W. SüdwestRothschild, Nov. Zool., v. 23, p. 301, 302 Holländisch(1916).

Neu-Guinea 
? M. al bertisi, W. Rothschild (\& Durrant), Rep. Brit. Un. Ornithol. Exp. Dutch N. Guinea, 2, Nr. 15, p. 18 (1915).

subsp. derhion Fruhst. M. $a$. d., Fruhstor- Westfer, Entom. Rundschau, v. 34, p. 43 (1917). Holländisch- $M$. a. „race $d$. (?), Joicey \& Talbot, Bull. Neu-Guinea Hill Mus., จ. 1, p. 352 (1922).

M. Albertisi, Fruhstorfer, Arch. Naturg., v. 81, 1915, Abt. A, Heft 11, p. 64 (1916).

subsp. aigion Fruhst. $M$. albertisi aigion, Waigeu Frubstorfer: A. Seitz, Großschmett. Erde, v. 9 , p. 409 (1911). - W. Rothschild, Nov. Zool., v. 23, p. 302 (1916).

Meeki Rothscb. \& Jord. M. m., W. Rothschild \& BritischK. Jordan, Nov. Zool., v. 12, p. 456 (1905). Süd- Fruhstorfer: A. Seitz, Großscbmett. Erre. จ. 9, p. 409 (1911). - W. Rothschild \& K. Jordan, Nov. Zool., v. 13, p. 759, t. 3, f. 1, 2 (1916). - K. Jordan, Proc. Ent. Soc. London, v. 5. II, p. 58 (1930).

nla Rothscb. \& Jord. M. u., W. Rothschild \& K. Neu-Guinea Jordan, Nov. Zool., v. 12, p. 454 (1905).

subsp. u. la Rothsch. \& Jord. $M$. u., W. Roth- Britiscbschild \& $\mathrm{K}$. Jordan, ut antea (1905). - W. Rothschild, Nov. Zool., v. 23, p. 301 (1916).

Neu-Guinea

(Owen

Stanley

Range)

subsp. $b r u n n i f a$ s c $i a$ Joicey, Noak. \& Talb. HolländischM. u. b., Joicey, Noakes \& Talbot, Trans. Ent. Soc. London, p. 366, t. 60, f. 1 (1915). - M. ula subsp., W. Rothschild, Nov. Zool., v. 23, p. 301 (1916). - ? M. u., Talbot, Proc. Ent. Soc. London, p. LXXXI (1921).

biakensis Joicey \& Talb. $M$. $b$., Joicey \& Talbot, Neu-Guinea Ent. Soc. London, p. 74 (1916).

subsp. bi a k en sis Joicey \& Talb. M. b., Joi- Nordostcey \& Talbot, ut antea, t. 5, f. 2 (1916). W. Rothschild, Nov, Zool., v. 23, p. 301,302 (part.), t. 4 , f. $3\left(\sigma^{\prime}\right)$ (non f. 4, Q: $=$ $M$. b. angustifascia) (1916).

M. Albertisi (non Oberthür) (part.), Grose Smith, Nov. Zool., v. 1, p. 357 (o') (sec. Rothschild, 1916) (1894). - Fruhstorfer: A. Seitz, Großschmett. Erde, v. 9, p. 409 (1911). subsp. angustifascia Joicey \& Talb. M. Meforb. a., Joicey \& Talbot, Bull. Hill Mus,, v. 1, p. 352 (1922).

Neu-Guinea

(Arfak-

Gebirge)

Neu-Guinea

(Biak,

Humboldt-

Bai ete.)

M. bia ke ensis, W. Rothschild, Nov. Zool., v.

(Mafor-)

23, p. 302 (part.), t. 4, f. 4 (q) (1916).

Phippsi Joicey \& Talb. M. p., Joicey \& Talbot, Bull. HolländischHill Mus., v. 1, p. 326 (1922).

Neu-Guinea

(Menoo-

Fluß,

Kunupi) 


\section{Subfamilia Discophorinae H. Stich.}

Discoph or idae, H. Stichel, Deutsch. Ent. Zeit. Iris, v. 15, p. 59 (1902). - Discophorinae, id., Gen. Ins., fasc. 31, p. 2 (1905). Discopterinae (!), D. Sharp, Zool. Record, Y. 42, 1905, pars 13, Insecta, p. 75 (1906). - Discopharinae, H. Stichel: A. Seitz, Großschmett. Erde, v. 1, p. 157 (1908). - Fruhstorfer, l. c., v. 9, p. 442 (1911). - Moulton, Journ. Straits Branch Asiat. Soc., Nr. 65, p. 18 (1913). - Godfrey, Journ. Nat. Hist. Siam, v. 2, p. 124 (1916). - Discopioridac, W. Rothschild, Nov. Zool., v. 23, p. 315 (1916). Discophorinae, id., Journ. Feder. Malay Stat. Mus., v. 8, p. 153 (1920).

Mo erae (Coitus) (part.), Jac. Hübner, Verz. Schmett., p. 51 [1818].

Elymninae (part.), Elwes (\& O. Möller), Trans. Fnt. Soc. London, p. 281 (1888).

\section{Genus Discophora Boisd.}

Boisduval, Spec. Gén. Lép., v. 1, Expl. Planches, p. 2; Discophorus, id., l. c., t. 4 (4A), f. 12 (1836). - Discophora, Lefobrre, Ann. Soc. Ent. France, v. 11, p. 11 (Morphol.) (1842). - (T. Doubleday \&) Westwood, Gen. Dium. Lep., v. 2, p. 329 (1851). - Chenu \& H. Lucas, Enc. Hist. Nat., Papill., p. 163 (1851). - Ménétriés, Enum. Carp. Anim. Mus. Petropol., pars 1, p. 37 (part.) (1855). - Horsfield \& F. Mroore, Descr. Cat. Lep. E.-Ind. Comp., v. 1, p. 211 (1857). - Westivool, Trans. Ent. Soc. London, n. ser., v. 4, p. 169,188 (1858). - Carus-Gerstäcker, Handb. Zool., v. 2, p. 232 (1863) . Herrich-Schäffer, Corr.-Bl. Zool--Min. Ver. Regensb., v. 18, p. 112; v. 19 , p. 88 (1864 \& 65). - A. G. Butler, Cat. Dium. Lep. Fabr. p. 45 (1869). - W. F. Kirby, Cat Diurn. Lep., p. 116, $\Lambda$ pu., p. 646 (1871). - S. H. Scudder, Proc. Amer. Ac. Sci., v. 10, p. 158 (Typ.: cclinde) (1875). - Piepers (\& P. O. T. Snellen), Tijdschr. Ent. v. 19, p. 148 (1876). - Wallace, Geogr. Distr. Anim., v. 1, p. 319 (1876). - W. F. Kirby, Entomologist, v. 10, p. 291 (1877). F. Müllor, Jena. Zeit. Naturw., v. 11, p. 102 (1877). - A. G. Butler, Trans. Linn. Soc. London, ser. 2, Zool., v. 1, p. 538 (1879). - W. F. Kirby, Sci. Proc. R. Dublin Soc., ser. 2, v. 2, p. $300(1880)_{2}-$ F. Moore, Lep. Ceylon, v. 1, p. 35 (1881) - C. Crüger (sec. Kirby, 1879), Berlin. Ent. Zeit., v. 25, p. 111 (1881). - Distant, Rhopal. Malay., p. 74, 426 (1882 \& 86). - B. Gerhard, Berlin. Ent. Zeit., v. 27, p. 181 (distr. geogr.) (1883). - G. F. L. Marshall \& Nicéville, Butt. Ind. Burma, Coyl., v. 1. II, p. 283, 294 (1883). - Kheil, Rhopal. Nias, p. 20 (1884). - Doherty, Journ. Asiat. Soc. Bengal, v. 55. II, p. 109 (1886). - E. Haase, Corr.-Bl. Ent. Ver. Iris; v: 1, p. 103, 310 (Organ. odor.) (1886 \& 88). - L. Glaser, Cat, Etymol. Col. Lep., p. 286 (spec. omiss.) (1887). - O. Staudinger (\& Schatz), Exot. Schmett., v. 1, p. 189 (1887). - G. Semper: C. Semper, Reis. Archip. Philipp., pars 2. V, Schmett., v. 1, p. 73 (1887). - E. Haase, Zool. Anzeig., v. 11, p. 479 (Organ. odor.). (1888). - Röber: O. Staudinger \& Schatz, Exot. Schmott., v. 2, t. 31 (Morphol.), p. 184, 186 (1888 \& 89). - Plateau (sec. E. Haase), Bull. Soc. Ent. Belg., p. LXI (1889). - Skertchly, Ann. Mag. Nat. Hist., ser. 6, v. 4, p. 212 (not. biol.) (1889). - B. Hagon, Tijdschr. Nederl, Aardr. Gen., ser. 2, v. 7, p. 200 (1890). - Doherty, Proc. Boston Soc. Nat. Hist., v. 25, p. 60 (1890) - Robbe, Ann. Soc. Ent. Belg., v. 36, p. 128 (1892). - Swinhoe, Trans. Ent. Soc. London, p. 275 (1893). - W. F. Kirby, Handb. Lep. (Allens Natural. Libr.), v. 1, 
p. 198 (1894). - F. Moore, Lep. Ind., ₹. 2, p. 187 (1895). Elera, Cat. Faun. Filippin,, v. 2, p. 271 (1895). - I. Martin, Deutsch. Ent. Zeit. Lep., v. 8, 1895, p. 262 (1896). - E. Reuter, Acta Soc. Sci. Fenn., v. 22, p. 109 (palpus) (1896). - Eimer (\& Fickert), Entstehg. d. Arten, pars 2, Orthogen., p. 178 (1897). A. Pagenstecher, Abh. Senckenb. Naturf. Ges., v. 23, p. 405 (1897). - K. Jordan, Nov. Zool., v. 5, p. 390, t. 15, f. 64 (antenna) (1898). - Fruhstorfer, Berlin. Ent. Zeit., v. 44, p. 59 (1899). - W. F. Kirby: Hübner \& Geyer, Samml. Exot. Schmett., Neue Ausg., v. 3, p. 46 (1901). - H. Stichel, Deutsch. Ent. Zeit. Iris, p. 60, 61, fig. 1, 2 (organ. copul.) (1902). - L. C. H. Young, Journ. Bombay Nat. Hist. Soc., v. 15, 1903, p. 298, 486 (1903 \& 04). - Shelford, Journ. Straits Branch Asiat. Soc., Nr. 41, p. 108 (1904). - H. Stichel, Gen. Ins., fasc. 31, p. 5, t., fig. 1 (Morphol.) (1905). Bingham, Faun. Brit. Ind., Butterfl., v. 1, p. 199 (Typ. D. celinde) (1905). - Bell, Tourn. Bombay Nat. Hist. Soc., v. 19, p. 468 (1909). - L. Martin, Mitt. Münch. Ent. Ges., v. 1, p. 71 (1910). Fruhstorfer: A. Bcitz, Großschmett. Erde, v. 9, p. 442 (1911). W. H. Evans, Journ. Bombay Nat. Hist. Soc., v. 21, p. 571 (1912). Moulton, Journ. Straits Branch Asiat. Soc., Nr. 65, p. 18 (1913). - W. H. Evans, Journ. Bombay Nat. Hist. ISoc., v. 29, p. 793, 796, t. 16, E 10 (1923). - Antram, Butt. Ind., p. 123 (1924). - Lo Corf, Enc. Ent., ser. B 3, Lep., v. 1, p. :141 (Morphol.) (1926). W. H. Evans, Identif. Ind. Butterfl., p. 87, t. 16, E 10 (1927). - A. Seitz, Entom. Rundschau, v. 45, p. 24 (1928).

Nymphalis (Gemmatus) Subgen. (part.), [D. H. Schneider], Nomencl. Entom., p. 36 (1785). - N. (part.), Gmelin-Linné, Syst. Nat., ed. 13, v. 1. V, p. 2307 (1790). Moera (part.), Jac. Hübncr, Verz. Schmett., p. 51 [1818]. - S. H. Scudder (sec. Hübner), Proc. Am. Ac. Sci., v. 10 , p. 220 (nom. praeocc.) (1875).

Morpho (part.), Latreille (\& Godart), Enc. Méth., v. 9, p. 11, 435; J. B. Godart, l. c., p. 446 (1819 \& 24). - Horsfield, Deser. Cat. Lep. Mus. E.-Ind. Comp., pars 2, Expl. Planches ad t. 6, p. '3, 4 (1829). - Verloren, Cat. Ins. Lep. Cramer, v. 2, p. 200 (1837). - M., Westwood: Donovan, Epitome Ins. Ind., n. ed., p. 44 (1842).

$Z$ erynthia (part.), Jac. Hübner, Samml. Exot. Schmett., v. 2 , t. 60 [1820-26].

Pota $m$ is (part.), Jac. Hübner, Cat. Lép. Coll. Franck, p. 79 [1826].

Th a umantis (part.), E. Blanchard, Hist. An. Artic., v. 3, p. $455,456(1840)$.

En is pe (part.), Horsfield \& F. Moore, Cat. Lep. Ins. Mus. E.-Ind. Comp., v. 1, p. 212 (1857). - Herrich-Schäffer, Corr.-Bl. Zool.-Min. Ver. Regensb., v. 19, p. 88 (1865). N. D. Riley \& Gabriel, Cat. Type Specim. Rhop. Brit. Mus., pars 1, p. 31 (1924).

\section{Sectio Eurypoecili}

II. Stichel, Gen. Ins., fasc. 31, p. 6 (1905).

\section{Cohors a. Celindiformes}

H. Stichel, ut antea, p. 6 (1905).

celinde (Stoll) "Celinde", Papilio c., C. Stoll, SundaSuppl. P. Cramer, Pap. Exot., p. 164, 382 Insoln (1790). - Discophora c., Fruhstorfer, Ber- 
lin. Ent. Zeit., v. 45, p. 13 (1900). - H. Stichel, Deutsch. Ent. Zeit. Iris, v. 15, p. 66,67 ; p. 62 , t. 1 , f. 1 (Organ. copul.) (1902). - Id., Gen. Ins., fasc. 31, p. 6 (1905). - Fruhstorfer: A. Seitz, Großschmett. Erde, v. 9, p. 445 (1911).

$\rightarrow$ subsp. celinde (Stoll) "Celinde", Papilio c., O. Stoll, ut antea, p. 164, t. 37, f. $1,1 \mathrm{~A}$ $\left(\sigma^{7}\right)$, p. 382 (1790). - Moera c., Jac. Hübner, Verz. Schmett., p. 51 [1818]. - Morpho c., (Latreille \&) J. B. Godart, Enc. Méth., v. 9, p. 446 (1824). - Horsfield, Deser. Cat. Lep. Mus. E.-Ind. Comp., pars 2 , t. 6, f. 6 ; t. 7, f. $11,11 \mathrm{a}-\mathrm{h}$ (larva chrysal., appendicos) (1829). - Discophora c., E. Doubleday, List Lep. Brit. Mus,, pars 1, p. 114 (1844). - Horrich-Schäffer, Samml. Außereur. Schmett., t. [4], f. 5, $6\left(\sigma^{7}\right)$; p. 54 (cit. part.), p. 77 (1850 \& 58) (conf. Dalla Torre, Ent. Nachr. Bl. Troppau, v. 1, 1927, p. 5, 58). - (E. Doubleday \&) Westwood, Gen. Diurn. Lep., v. 2, p. 331 (part.) (1851). - Chenu \& H. Lucas, Enc. Hist. Nat., Papill., p. 163, t. 39, f. 1 (q) (distr. part.) (1851). - Ménétriés, Enum. Corp. Anim. Mus. Petropol., pars 1, p. 37 (1855). - Westwood, Trans. Ent. Soc. London, n. ser., v. 4, p. 164, 165 (larva sec. Horsfield) (1856). - Horsfield \& F. Moore, Cat. Lep. Mus. E.-Ind. Comp., v. 1, p. 211, t. 6, f. 5 , $5 a$ (larva, chrysal.) - (1857). - Herrich-Schäffer, Corr.-Bl. Zool--Min. Ver. Regensb., v. 19, p. 88 (sine synon.) (1865). - A. G. Butler, Cat. Diurn. Lep. Fabr., p. 45 (sine synon.) (1869). - W. F. Kirby, Cat. Diurn. Lep., p. 116 (cit. part.) (1871). - Piepers (\& P. O. T. Snellen), Tijdschr. Ent., v. 19, p. 148 (1876). - Distant, Rhopal. Malay., p. 76 (part.); p. 167, f. 19 (larva sec. Horsfield) (1882), - Wilh. Müller, Zool. Jahrb., v. 1, p. 611 (larva sec. Horsf.-Moore) (1886). - O. Staudinger (\& Schatz), Exot. Schmett., v. 1, p. 190 (part.) (non t. $63:=D$. continentalis) ' $(1886 \&$ 87). - A. Pagenstecher, Jahrb. Nassau. Ver. Naturk., v. 43, p. 96 (1890). - E. Hofmann, Jahresh. Ver. Naturk. Württemb., v. 46, p. 236 (Physiol., Organ. odor.) (1890). - Nicéville, Journ. Bombay Nat. Hist. Soc., v. 7, p. 326 (synon. part.) (1892). - F. Moore, Lep. Ind., v. 2, p. 196 (cit. part.) (1895). - Fürbringer (\& A. Pagenstecher): Semon, Zool. Forschungsr. 5, Denkschr. Med.Naturw. Ges. Jena, v. 8, p. (78) 238 (1895). - Kobus, Tijdschr. Ent., v. 39, p. 115, t. 3, f. $1-5$ (Biol.) (1897). - Fruhstorfer, Berlin. Ent. Zeit., v. 41, 1896, p. 301 (1897). - Eimer (\& Fickert), Entstehg. d. Arten, pars 2, Orthog., p. 178, 235 (1897). -

Java,

Bali,

Kangean-

Inseln 
Nicéville \& Elwes, Journ. Asiat. Soc. Bengal, v. 66. II, 1897 , p. 681 (1898). - D. c. c., Fruhstorfer, Berlin. Ent. Zeit., v. 45, p. 13 (part.) (1900). - D. c., H. Stichel, 1. c., v. 46, Sitz.-Ber., p. (4) (1901) - D. c. (typica), id., Deutsch. Ent. Zeit. Iris, v. 15, p. 69 (1902). - D. c., P. O. T. Snellen, Tijdschr. Ent., v. 45, p. 80 (1902). - D. c. c., H. Stichel, Gen. Ins., fasc. 31, p. 6 (1905). - Fruhstorfer: A. Seitz, Großschmett. Erde, v. 9, p. 446 , t. $106 \mathrm{~b}\left(\sigma^{\prime}\right.$, १) (1911). - W. H. Evans, Journ. Bombay Soc. Nat. Hist., v. 22, 1913, p. 763 (1914).

menetho (Fabr.) Papilio m. J. O. Fabricius, Ent. Syst., v. 3. I, p. 83 () (1793). - Donovan, Epitome Ins. Ind., t. [30], $f$. 1 (1800). - Turton, Gen. Syst. Nat. Liuné, v. 3. II, p. 87 (1806). - Morpho c., (Latreille \&) J. B. Godart, Enc. Méth., v. 9, p. 446 (patria falsa) (1824). - Discophora m. Boisduval, Spec. Gén. Lép., จ. 1, Expl. Planches, p. 2, t. 4, f. 12 (larva, chrysal. sec. Horsfield) (1836). - Morpho (Thaumantis) m., E. Blanchard, Hist. An. Artic., v. 3, p. 456 (1840). - M. m., Westwood: Donovan, Epitome Ins. Ins., n. ed., t. 30 , f. 1, p. 44 (1842). - Discophara $m$, G. Semper: C. Semper, Reis. Arch. Philippin., pars 2. V. Schmett., v. 1, p. 74 (in cit.) (1887). - ? Niceville, Journ. Bombay Nat. Hist. Soc., v. 7, p. 326 (patria falsa) (1892).

aristides (Fabr.) Papilio a., J. O. Fabricius, Ent. Syst., v. 3. I, p. 86 (ㅇ) (1793). - Turton, Gen. Syst. Nut. Linné, v. 3. II, p. 87 (1806).

D. $z a l$ (non Westwood), W. F. Kirby, Oat. Diurn. Iep., p. 116 (in cit.) (1871).

A mathusia phidippus (non Pap. $p$. Johansson-Linné), (Marshall \&) Nicéville, Butt. Ind. Burm. Ceyl., v. 1. II, p. 290 (larvae descr. solum, conf. F. Moore, Lep. Ind., v. 2, 1895, p. 179, nota) (1883).

forma $v a r d a$ Fruhst. $D_{\text {. }}$ c. c. forma v., Typ.: Java Fruhstorfer: A. Seitz, Großschmett. Erde, v. 9 , p. 446 (1911).

subsp. undata Stich. D. c. u., H. Stichel, Lombok Deutsch. Ent. Zeit. Iris, v. 15, p. 68, 70 (1902). - Id., Gen. Ins., fasc. 31, p. 7 (1905). - Fruhstorfer: A. Seitz, Großschmett. Erde, v. 9, p. 446 (1911).

D. timora (non Westwood), Fruhstorfer, Berlin. Ent. Zeit., v. 42, p. 4 (1897). Nicéville, Journ. Asiat. Soc. Bengal, v. 66. II, 1897 , p. 681 (1898). - D. celinde $t$., Fruhstorfer, Berlin. Ent. 'Zeit., v. 45, p. 13 (1900).

subsp. $t$ im or a Westwood D. $t$., (E. Doub- Timor leday \&) Westwood, Gen. Diurn. Lep., t. 54 , f. 2 (ㅇ); v. 2, p. 331 (geogr. var. 
D. celinde?) (1849 \& 51). - O. Staudinger (\& Schatz), Exot. Schmett., v. 1, p. 190 (1887). - Doherty, Journ. Asiat. Soc. Bengal, v. 60. II, p. 170 (1891). - F. Mogre, Lep. Ind., v. 2, p. 197 (1895). - D. celinde t., Fruhstorfer, Berlin. Ent. Zeit., v. 45, p. 13 (part.) (1900). - N. D. Riley \& Gabriel, Cat. Type Specim. Rhop. Brit. Mus., par 1, p. 59 (1924). (Subsp. dubia!)

D. c el inde var. O? D. timora, Horsfield \& F. Moare, Cat. Lep. Mís. E.-Ind. Comp., v. 1, p. 211 (1857). - D. c. var. a." D. timora, W. F. Kirby, Cat. Diurn. Lep., p. 116 (1871). - D. c., Nicéville, Journ. Bombay Nat. Hist. Soc., จ. 7, p. 326 (in cit.) (1892). - H. Stichel, Deutsch. Ent. Zeit. Lris, v. 15, p. 68 (in cit.) (1902). - Id., Gen. Ins., v. 31 , p. 7 (1905).

continentalis Stauding. $D$. celinde var. continentalis, NordO. Staudinger (\& Schatz), Exot. Schmett., v. 1, Indien p. 190 (1887). - D. continentalis, H. Sti- bis chel, Deutsch. Ent. Zeit. Iris, v. 15, p. $66,67,70 ;$ p. 62 , t. 1 , f. 2 (organ. copul.) (1902). - Id., Gen. Ins., fasc. 31, p. 7 (1905). - Frubstorfer: A. Seitz, Großschmett. Erde, v. 9, p. 445 (1911). subsp. continental is Stauding. $D$. cc-
linde var. contincntalis, O. Staudinger (\& Schatz), ut antea, t. 63 (cclindc) (1887 \& 86). - Nicéville, Journ. Bombay Nat. Hist. Soc., v. 7, p. 326 (18992). - D. continentalis, F. Moore, Lep. Ind., v. 2, p. 188 (part.), t. 150, f. 1 a, b $\left(O^{\prime \prime}\right), 1$ c (\$), larra, chrysal. (1895). - E. Reuter, Acta Soc. Sci. Fenn., v. 22, p. 109 (palpus) (1896). - D. celinde continentalis, Fruhstorfer, Berlin. Ent. Zeit., v. 45, p. 13 (1900). - D. cel. var. cont., H. Stichel, l. c., v. 46, Sitz-Ber., p. (4) (1901). D. continentalis (typica), id., Deutsch. Ent. Zeit. Iris, จ. 15, p. 71 (1902). - D. c. c., id., Gen. Ins., fasc. 31, p. 7 (1905). Fruhstorfer: A. Seitz, Großschmett. Lirde, v. 9, p. 445 (1911). - D. celinde ,race continentalis, W. H. Evans, Journ. Bombay Nat. Hist. Soc., v. 21, p. 571 (1912). $D$. cel. cont., id., l. c., v. 22, 1913, p. 763 (1914). - E. V. Ellis, l. c., v. 25, p. 107 (1917). - Ollenbach, l. c., v. 27, p. 887 (1921). - D. contincntalis c., W. H. Evans, l. c., v. 29, p. 797 (1923). - Id., Identif. Ind. Butterfl., p. 87 (in synon. semincchis pro seminecho, sed. errans!) (1927).

D. celindc (non Papilio c. Stoll), Horsfield \& F. Moore, Cat. Lop. Ins. Mus. E.Ind. Comp., v. 1, p. 211 (part.) (1857). - D. c., F. Moore, Proc. Zool. Soc. Iondon, p. 761 (1865). - Nicéville, Journ.

Annam,

Malayische

Halbinsel,

? Sumatra

Nepal-

Bhutan,

Assam,

Burma,

Siam,

Tonkin,

Annam,

Tenasserim,

? Malakka,

Mergui 
Asiat. Soc. Bengal, v. 50. II, p. 57 (part.) (1881). - Id., 1. c., v. 51. II, p. 57 (part.) (1882). - Distant, Rhop. Malay., p. 75 (cit. et distr. part.), t. 5, f. 10 $\left(\sigma^{\top}\right)$; non fig. $11,0=D$. necho engamon) (1882). - G. F. L. Marshall \& Nicéville, Butterfl. Ind. Burma, Ceyl., v. 1. II, p. 295 (cit. et distr. part.), fig. ơ, o (1883). - ? F. Moore, Journ. Linn. Soc. London, Zool., v. 21, p. 33 (1886).Elwes (\& O. Möller), Trans. Ent. Soc. Iondon, p. 281, 331 (1881), - Nicéville, Gazetteer Sikhim, List Butterfl., p. 130 (1894). - Bingham, Faun. Brit. Ind., Butterfl., v. 1, p. 200 (cit. part.) (1905). Bell, Journ. Bombay Nat. Hist. Soc., v. 19, p. 467, 469 (Biol.) (1909). - Antram, Butterfl. Ind., p. 123, fig. 252 (q) (1927). D. $m$ enetho (non Papilio $m$. Fabricius), A. G. Butler, Cat. Diurn. Lep. Fabr., p. 45 (1869). - ? Id., Trans. Ent. Soc. London, ser. 2, Zool., v. 1, p. 538 (an D. c. perakensis) (1879).

? D. necho (non Felder), F. Moore, Proc. Zool. Soc. London, p. 827 (cit. except.) (1878). - ? Elwes \& Nicéville, Journ. Asiat. Soc. Bengal, v. 55. II, 1886, p. 419 (1887).

D. timora t., (non Westwood), Godfrey (sec. Riley in litt.), Journ. Siam Soc. Nat. Hist. Suppl., v. 7, p. 263 (1930).

forma seminecho Stich. D. continentalis ab. s., H. Stichel, Deutsch. Ent. Zeit. Lep. Iris, v. 15, p. 71,73 (1902). - D. c. c. forma s., H. Stichel, Gen. Ins., fasc. 31, p. 7 (1905). - D. c. s., Fruhstorfer: A. Seitz, Großschmett. Erde, V. 9, p. 445 (1911). D. c. ,race" s., W. H. Evans, Journ. Bombay Nat. Hist. Soc., จ. 22, 1913, p. 763 (1914). - D. c. 8., Godfrey, Journ. Nat. Hist. Soc. Siam, v. 2, p. 124 (1916). - Id, Journ. Feder. Malay Stat. Mus., v. 3, p. 467 (1919).

D. necho (non Felder), G. F. L. Marshall \& Nicéville, Butt. Ind. Burma Ceyl., v. 1. II, p. 296 (cit. except., distr. part.) (1883).

D. celinde (non Papilio c. Stoll), Bingham, Faun. Brit. Ind., Butterfl., v. 1, p. 200 (in cit.) (1905).

subsp. perakensis Stich. D. p., H. Sti- Perak, chel, Ins.-Börse, v. 17, p. 259 (1900). - ? Sumatra Id., Berlin. Ent. Zeit., v. 45, Sitz.-Ber., p. (21) (1901). - D. continentalis p., id., Deutsch. Ent. Zeit. Iris, v. 15, p. 71, 74 (1902). - Id., Gen. Ins., fasc. 31, p. 7 (1905). - - Fruhstorfer: A. Seitz, Großschmett. Erde, v. 9, p. 445 (1911). 
? D. menetho (non Papilio m. Fabr.), A. G. Butler, Trans. Linn. Soc. London, ser. 2, Zool., v. 1, p. 538 (conf. D. continentalis c.) (1879).

P D. celinde (non Papilio c. Stoll), B. Hagen, Deutsch. Ent. Zeit. Lep., v. 9, p. 154 (1896).

subsp. andamensis Stauding. D. celinde. Andamanen

a., O. Staudinger (\& Schatz), Exot. Schinett.,

v. 1, p. 190 (1887). - Nicéville, Journ.

Bombay Soc. Nat. Hist., v. 7, p. 326 (1892). - D. c. andamanensis, Fruhstorfer, Berlin. Ent. Zeit., v. 45, p. 13 (1900). - D. continentalis andamensis, H. Stichel, Deutsch. Ent. Zeit. Iris, v. 15, p. 71, 73 (1902). Id., Gen. Ins., fasc. 31, p. 7 (1905). Frubstorfer: A. Seitz, Großschmett. Erde, v. 9 , p. 445 (1911). - D. celinde ,raco" $a_{\text {.s }}$ W. H. Evans, Journ. Bombay Nat. Hist. Soc., v. 21, p. 571 (1912). - D. continentalis a., id., l. c., v. 29 , p. 797 (1923). Id., Identif. Ind. Buttorfl., p. 87 (1927). D. celinde (non Papilio c. Stoll), F. Moore, Proc. Zool. Soc. London, p. 583 (1877). Wood-Mason \& Nicéville, Journ. Asiat. Soc. Bengal, v. 50. II, p. 245 (1881). - F. Moore, Lep. Ind., v. 2, p. 188 (in cit.) (1895). Bingham, Faun. Brit. Ind., Butterfl., v. 1, p. 200 (in cit.) (1905).

deo Nicév. D. a., Nicéville, Journ. Bombay Nat. HinterHist. Soc., v. 12, p. 137 (1898). - H. Sti- Indien chel, Deutsch. Ent. Zeit. Iris, v. 15, p. 66, 83 ; p. 62 , t. 1 , f. 12 (organ. copul.) (1902). - Id., Gen. Ins., fasc. 31, p. 7 (1905). - Frulistorfer: A. Seitz, Großschmett. Erde, v. 9, p. 444 (1911).

subsp. d eo Nicév. D. d., Nicéville, ut antea, Assam, t. $Y$, fig. 10 (1898). - D. d. (typica), H. Burma, Stichel, Deutsch. Ent. Zeit. Iris, v. 15, p. Schan84 (1902). - D. d. d., id., Gen. Ins., fasc. Staaten, 31, p. 7 (1905). - Fruhstorfer: A. Seitz, Großschmett. Erde, v. 9, p. 444 (1911). D. d., W. H. Evans, Journ. Bombay Nat. Hist. Soo., v. 21, p. 571 (1912). - Tytler, 1. c., v. 23 , p. 229 (1914). - W. H. Evans, l. c., v. 29, p. 797 (1923). - Id., Identif. Ind. Butterfl., p. 87 (1927). - D. d. d., Godfrey, Journ. Siam Soc. Nat. Hist. Suppl., v. 7, p. 263 (1930).

- subsp. Fruhstorferi Stich. D. d. f., H. Tonkin Stichel, Ins.-Börse, v. 18, p. 317 (1901). Id., Deutsch. Ent. Zeit. Iris, v. 15, p. 84 (1902). - Id., Berlin. Ent. Zeit., v. 47, Sitz.-Ber., p. (11) (1902). - Id., Gen. Ins., fasc. 31, p. 7 (1905). - Fruhstolfer: A. Seitz, Großschmett. Erde, v. 9, p. 444 (in marg. "ruhstorferi": err. typ., corr. p. 1111) (1911). 


\section{Cohors b. Nechoiormes}

H. Stichel, Gen. Ins., fasc. 31, p. 8 (1905).

necho Feld. D. n., O. \& R. Felder, Reise Novara, Sundav. 2. II, p. 462 (1866). - H. Stichel, Inseln, Deutsch. Ent. Zeit. Iris, v. 15, p. 65, 67, Malakka, 75 ; p. 61, 62, fig. 2, 3 a, b (organ. copul.) Philippinen (1902). - Shelford, Journ. Straits Branch Asiat. Soc., Nr. 41, p. 107 (1904). - H. Stichel, Gen. Ins., fasc. 31, p. 8 (1905). Fruhstorfer: A. Seitz, Großsschmett. Ende, v. 9, p. 446 (1911).

- subsp. necho Feld. D. n., O. \& R. Felder, Java ut antea (1866). - W. F. Kirby, Cat. Diurn. Lep., p. 116 (1871). - O. Staudinger (\& Schatz), Exot. Schmett., v. 1, p. 190 (1887). - Nicéville, Journ. Bombay Nat. Hist. Soc., v. 7, p. 326 (1892). - F. Moore, Lep. Ind., v. 2, p. 197 (1895). H. Stichel, Berlin. Ent. Zoit., v. 46, Sitz.Ber., p. (5) (1901). - D. n. (typica), id., Deutsch. Ent. Zeit. Iris, v. 15, p. 75; p. 61, 62 (organ. copul.) (1902). - D. n. n., id., Gen. Ins., fase. 31 , p. 8 (1905). Fruhstorfer: A. Seitz, Großschmett. Erde, v. 9, p. 446 (1911).

D. ogina, (Latreille \&) J. B. Godart, Enc. Méth., v. 9, p. 445 (part.) (1824).

D. $d i s$ (non Nicéville), Fruhstorfer, Berlin. Ent. Zoit.s v. 41, 1896, p. 301 (1897).

- subsp. cheops Feld. D. c., C. \& R. Felder, NordReise Novara, v. 2. II, p. $462(1866)$. $-D$. Borneo necho var, a. D. c., W. F. Kirby, Cat. Diurn. Lep., p. 116 (1871). - Herb. Druce, Proc. Zool. Soc. London, p. 341 (1873). $D$. var. c., D. c., E. Haase, Corr.-Bl. Int. Ver. Iris, v. 1, p. 103, 310 (organ. odor.) (1886 \& 88). - D. c., O. Staudinger (\& Schatz), Exot. Schmett., v. 1, p. 190 (part.) (1887). - Distant \& Pryer, Ann. Mag. Nat. Hist., ser. 5, v. 19, p. 51 (1887). - Nicéville, Journ. Bombay Nat. Hist. Soc., v. 7, p. 326 (1892). - F. I Ioore, Lep. Ind., v. 2, p. 197 (1895). - K. Jordan, Nov. Zool., v. 5, p. 390 , t. 15 , f. 64 (antenna) (1898). - D. c. c., Fruhstorfer, Berlin. Ent. Zeit., v. 45 , p. 13 (part.) (1900). - D. c., H. Stichel, 1. c., v. 46, Sitz,-Ber., p. (5) (necho- Form) (1901) - D. c. c., Id., Deutsch. Ent. Zeit. Iris, v. 15, p. 75, 76 (part.) (1902). - D. necho c., Shelford, Journ. Straits Branch Asiat. Soc., Nr. 41, p. 108 (1904). - H. Stichel, Gen. Ins., fasc. 31, p. 8 (part.) (1905) - Fruhstorfer: A. Seitz, Großschmett. Erde, v. 9, p. 446 (1911). - Moulton, Journ. Straits Branch Asiat. Soc., Nr. 65, p. 18 (1913).

? D. $d i s$, Nicéville, Journ. Bombay Sac. Nat. Hist., v. 7 , p. 325 (part.) (1892). 
subsp. helvidius Fruhst. D. n. h., Fruh- Südstorfer: A. Seitz, Großschmett. Erde, v. 9, Borno p. 447 (1911).

D. n. cheops (part.) Fruhstorfer, Berlin. Ent. Zeit., V. 45, p. 13 (1900). - H. Stichel, Deutsch. Ent. Zeit. Iris, v. 15, p. 76 (1902). - Id., Gren. Ins., fasc. 31, p. 8 (1905).

forma orbicularis Stich. $D$. necho che- Typ.: Südops ab. o., H. Stichel, Deutsch. Ent. Zeit. Borneo Iris, v. 15, p. 75, 78 (1902). - Fruhstor- (Bandjerfer: A. Seitz, Großschmett. Erde, v. 9, p. masin) 447 (1911).

- subsp. $d i_{s}$ Nicév. $D$. d., Nicéville, Journ. Sumatra Asiat. Soc. Bengal, v. 7, p. 325 (not. biol., distr. part.), t. H. fig. 3 (1892). - F. Moore, Lep. Ind., v. 2, p. 197 (1895). Fruhstorfer, Berlin. Ent. Zeit., v. 45, p. 13 (1900). - D. necho d., id., Großschmett. Erde, v. 9, p. 447, t. 106d (o, \&) (1911). W. Rothschild, Journ. Feder. Malay Stat. Mus., v. 8, p. 154 (1920).

D. n. var. cheops (non D. c. Felder), B. Hagen, Tijdschr. Nederl. Aardr. Gen., ser. 2, v. 7, p. 199 (1890). - D. C., L. Martin, Deutsch. Ent. Zeit. Lep., v. 8, 1895, p. 263 (1896).

D. necho, Nicéville \& L. Martin, Journ. Asiat. Soc. Bengal, v. 64 . II, 1895 , p. 395 (not. biol.) (1896).

subsp. propinqua Stich. D. $n$. p., H. Sti- Nias

chel, Ins.-Börse, v. 17, p. 69 (1900). - - Id., Berlin. Eut. Zeit., v. 46, Sitz.-Ber., p. (5) (1901). - Id., Deutsch. Ent. Zeit. Iris, v. 15 , p. 75,78 (1902). - Id., Gen. Ins., fasc. 31, p. 8 (1905). - Fruhstorfer: A. Seitz, Großschmett. Erde, v. 9, p. 446 (1911).

D. cheops (non Felder), Kheil, Rhopal. Nias, p. 20 (1884).

subsp. engam on Fruhst. D. n. e., Fruh- Perak storfer: A. Seitz, Großschmett. Ende, v. 9, p. 155 (1911).

D. celinde (non Papilio c. Stoll), Distant, Rhopal. Malay., p. 75 (part.), t. 5, f. 11 (Q) (conf. D. continentalis) (1882).

subsp. odora Fruhst. D. n. o., Fruhstor- Palawan fer, Berlin. Ent. Zeit., v. 45, p. 13 (1900). - H. Stichel, Deutsch. Ent. Zeit. Iris, v. 15, p. 75,78 (part.) (1902). - D. n. o. (D. c. var. palavanicus O. Staudinger MS), id., Gen. Ins., fasc. 31, p. 8 (part.) (1905). - Fruhstorfer: A. Seitz, Großschmett. Erde, v. 9, p. 447 , t. $106 a$ (odorata on, $q$, corr. p. 1135) (1911 \& 27).

odorata Fruhst. D. o., Fruhstorfer, ut antea, (1911).

D. cheops (non Felder), O. Staudinger, Deutsch. Ent. Zoit. Lep., v. 2, p. 42 (1889).

- G. Semper: O. Semper, Reis. Arch. Phi- 
lippin., pars 2. V, Schmett., v. 1, p. 333 (part.) (1892). - ? Elera, Cat. Faun. Filipin., v. 2, p. 271 (part., cit. except.) (1895).

subsp. mindorana Fruhst. $D . \quad n$. m., Mindoro, Fruhstorfer: A. Seitz, Großschmett. Ende, P Luzon, v. 9 , p. 447 (1911).

D. cheops (part.), G. Semper, ut antea (1892). - ? Elera, ut antea (1895).

$D$. necho od ora (part.), D. c. var. mindorana Stauding. MS), H. Stichel, ut antea $(1902 \& 05)$.

subsp. erasimus Fruhst. D. n. e., Fruh- Jolostorfer: A. Seitz, Großschmett. Erde, v. 9, (Sulu-) p. 447 (1911).

Polillo

philippina Moore $D_{\text {. }}$ p., I. Moore, Lep. Ind., v. 2, Mindanao p. 198 (1895). - D. necho p., Fruhstorfer, Bohol, Berlin. Ent. Zeit., v. 45, p. 13 (1900). - Camotes, D. p., H. Stichel, Deutsch. Ent. Zeit. Iris, Basilan v. 15 , p. $65,66,81$, t. 2 , f. 3 (q); p." 62 , t. 1 , f. 6 (organ. copul.) (1902). - Id., Gen. Ins., fasc. 31 , p. 8 (1905). - Truhstorfer: A. Seitz, Großschmett. Erde, v. 9, p. 447 (1911).

D. menetho (non Papilio $m$. Fabricius), G. Semper: C. Semper, Reis. Arch. Philippin., pars 2. V, Schmett., v. 1, p. 74 (cit. except.), t. 14, f. 1, 2 (1887). - Elera, Cat. Faun. Filipin., v. 2, p. 271 (1895).

\section{Cohors c. Oginiformes}

H. Stichel, Gen. Ins., fasc. 31, p. 8 (1905).

ogina (God.) Morpho o., (Latreille \&) J. B. Go- Philinninen dart, Enc. Méth., v. 9, p. 445 (part.) (pa- (Luzon, tria falsa: Java) (1824). - Zerynthia o., Polillo) Jac. Hübner, Samml. Exot. Schmett., v. 2, t. [60], f. 1, 2 [1819-26]. - Potamis o., id., Cat. Lép. Franck, p. 79 [1826].Morpho (Tharmantis) agina (!), E. Blanchard, Hist. An. Artic., v. 3, p. 456 (1840). - Discophora o., (E. Doubleday \&) Westwood, Gen. Diurn. Lep., v. 2, p. 331 (patria falsa) (1851). - Chenu, \& H. Lucas, Enc. Hist. Nat., Papill., p. 163 (patria falsa) (1851). - Herrich-Schäffer, Corr.Bl. Zool.-Min. Ver. Rogensb., v. 19, p. 88 (1865). - W. F. Kirby, Cat. Diurn. Lep., p. 117 (patria falsa) (1871). - P Hewitson, Ann. Mag. Nat. Hist., ser. 4, v. 14, p. 356 (patria falsa: Andaman, aut determ. errans) (1874). - O. Staudinger (\& Schatz), Exot. Schmett., v. 1, p. 190 (1887). - G. Semper: C. Scmper, Reis. Arch. Philippin., pars 2. V, Schmett., v. 1, p. 74, t. 13, f. 8,9 (q), p. 333 (1887 \& 92). Nicéville, Journ. Bombay Nat. Hist. Soc., 
v. 7, p. 326 (1892). - F. Moore, Lep. Ind., v. 2, p. 197 (1895). - Elera, Cat. Faun. Filipin., v. 2, p. 271 (1895). - Zerynthia o., Discophora o., W. F. Kirby: Hübner \& Geyer, Samml. Exot. Schmett., Neue Ausg., v. 2 , t. $273(60)$, f. 1, 2; v. 3, p. 46 (=D. melinda $)(1898 \& 1901) .-D$. o., Fruhstorfer, Berlin. Ent. Zeit., v. 45, p. 14 (1900), - H. Stichel, Deutsch. Ent. Zeit., Iris, v. 15 , p. $65,67,79$; p. 62 , t. 1 , f. 5 (organ. copul.) (1902). - Id., Gen. Ins., fasc. 31, p. 8 (1905). - Frulistorfer: A. Seitz, Großschmett. Erde, v. 9, p. 447 (1911).

forma melind a Feld. D. m., O. \& R. Felder, Wien. Ent. Monatschr., v. 7, p. '122 (Q) (1863). - Herrich-Schäffer, Corr.-Bl. Zool.-Min. Ver. Regensb., v. 19, p. 88 (1865). - W. F. Kirby, Cat. Diurn. Lep., p. 116 (1871). - O. Staurlinger (\& Schatz), Exot. Schmett., v. 1, p. 190 (1887). - D. O. o. „Form" m., Fruhstorfer: A. Seitz, Großschmett. Erde, v. 9, p. 447 (1911).

D. o gina (part.), Nicéville, Journ. Bombay Nat. Hist. Soc., v. 7, p. 326 (in cit.) (1892). - F. Moore, ut antea (1895). - H. Stichel, ut antea $(1902,1905)$.

bambusae Feld. D. b., O. \& R. Felder, Reise Novara, v. 2. II, p. 462 (1866). - Fruhstorfer: A. Seitz, Großschmett. Erde, v. 11, p. 447 (1911).

subsp. $b a m b u s a e$ C. \& R. Felder, ut antea (patria falsa: Halmahera) (1866). - W. F. Kirby, Cat. Diurn. Lep., p. 116 (1871). - O. Staudinger (\& Schatz), Exot. Schmett., v. 1, p. 190 (1887). - Nić́ville, Journ. Bombay Nat. Hist. Soc., v. 7, p. 326 (1892). F. Moore, Lop. Ind., v. 2, p. 198 (1895). Fruhstorfer, Berlin. Ent. Zeit., v. 45, p. 14 (? Molukken!). (1900). - H. Stichel, Deutsch. Ent. Zeit. Iris, v. 15, p. $65,67,80$ (part.); p. 62, t. 1, f. 4 (organ. copul.) (1902). Id., Gen. Ins., fasc. 31, p. 9 (part.) (1905). - D. b. b., Fruhstorfer: A. Seitz, Großschmett. Ende, v. 9, p. 447 , t. 106 b (Q) (1911).

2- subsp. celebensis Holland D. c., Holland, Sï Proc. Boston Soc. Nat. Hist., v. 25, p. 59, t. 5 , f. 5,6 (D. ogin $x$ local moe?) (1890). - W. Rothschild, Deutsch. Ent. Zeit. Lep., v. 5, p. 434 (1892). - F. Moore, Lop. Ind., v. 2, p. 197 (1895). - D. bambusae c., Fruhstorfer, Berlin. Ent. Zeit, v. 45, p. 14 (part.) (1900). - Id.: A. Seitz, Großschmett. Erde, v. 9, p. 447 (1911). (Subspec. dubia).

D. $b a m b u s a e$ (part.), H. Stichel, ut antea $(1902,1905)$.

Typ.: Luzon

Celebes,

Bangkai

Nord-

Celebes

Celebes

(Macassar) 
subsp. bangkaiensis Fruhst. D. bambu'sae bangkaiensis, Fruhstorfer, Soc. Entom., v. 16, p. 82 (1902). 一 Id.: A. Seitz, "Großschmett. Erde, v. 9, p. 418 (1911).

D. $b a m b u s a e$ (part.), H. Stichel, ut antea $(1902,1905)$.

lepida (Moore) Enispe l. (Horsfield \&) F. Moore, VorderCat. Lop. Ins. Mus, E.-Ind. Comp., v. 1, Indien p. 213 (1857). - Discophora l., H. Stichel, Deutsch. Ent. Zeit. Iris, v. 15, p. 66, 67,82 ; p. 62 , t. 1 , f. 7 (organ. copul.) (1902). - Id., Gen. Ins., fasc. 31, p. 9 (1905). - Fruhstorfer: A. Seitz, Großschmett. Erde, v. 9, p. 445 (1911).

subsp. le pida (Moore) Enispe l., (Hors- Südliches field \&) F. Moore, ut antea, p. 213 (Q) (1857), - Horrich-Schäffer, Corr.-Bl. Zool.Min. Ver. Regensb., v. 19, p. 88 (1865). Britisch W. F. Kirby, Cat. Diurn. Lep., p. 117 (1871). - Discophora l., F. Moone, Lep. Coylon, p. 36 (part.) (non t. 18, f. 1, $1 \mathrm{a}$, b: $=$ D. l. ceylonica) (1880). - WoodMason, Journ. Asiat. Soc. Bengal, v. 50. II, p. 86 (1881). - G. F. L. Marshall \& Nicéville, Butt. Ind. Burm. Coyl., v. 1, p. 297 (part.) (1882). - O. Staudinger (\& Schata), Exot. Schmett., v. 1, p. 190 (part.) (1887). G. F. Hampson, Journ. Asiat. Soc. Bengal, v. 57, II, 1888, p. 351 (1889). - E. Y. Watson, Journ. Bombay Nat. Hist. Soc., v. 5, p. 31 (1890). - Ferguson, 1. c., v. 6, p. 437 (not. biol.) (1891). - F. Moore, Lep. Ind., v. 2 , p. 190, t. 151, f. $1,1 \mathrm{a}-\mathrm{c}(\sigma, Q)$ (1895). - Davidson, Bell \& Aitken, Journ. Bombay Nat. Hist. Soc., v. 10, p. 245 (Biol.) (1896). - Nićville, Journ. Asiat. Soc. Bengal, v. 69. II. p. 220 (nutr. larvae) (1900). - D. lepidea (!), Fruhstorfer, Berlin. Ent. Zeit., v. 45 , p. 13 (part.) (1900) - D. lepida (typica), H. Stichel, Deutsch. Ent. Zeit. Iris, v. 15, p. 82 (part.) (1902). D. l., L. C. H. Young, Journ. Bombay Nat. Hist. Soc., v. 15, 1903, p. 486 (1904). Bingham, Faun. Brit. Ind., Butterfl., v. 1, p. 201 (part.) (1905). - H. Stichel, Gen. Ins., fasc. 31 , p. 9 (part.) (1905). - Bell, Journ. Bombay Nat. Hist. Soc., v. 19, p. 35 (Androcon.); p. 467 (sine synon.), p. 469, 470 , (Biol.), t. 1 , f. 3 (larva), f. $3 a$ (chrysal.) (1909). - W. H. Evans, Journ. Bombay Nat. Hist. Soc., v. 20, p. $382(1910)$. - D. l. C., Fruhstorfer: A. Seitz, Großschmett. Erdo, v. 9, p. 445 , t. 105 c (non t. $106 \mathrm{~d}:=D . l$. ceylonica)

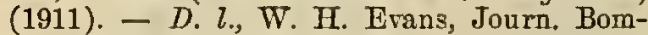
bay Nat. Hist. Soc., v. 21, p. 571 (part.); จ. 22,1913, p. $763(1912 \& 14)$ - — D. l. l., id., 1. c., v. 29, p. 797 (part.), t. 16, fig. E 10. 3 (1923). - Enispe l., N. D. Riley, Cat. Type Specim. Rhop. Brit. Mus., pars 
1, p. 31 (1924). - Discophora l., Antram, Butt. Ind., p. 123, f. 253 (1924). - D. l. l., W. H. Evans, Identif. Ind. Butterfl., p. 87 (part.), t. 16, fig. E 10.3 (1927). - D. l., Yates, Journ. Bombay Nat. Hist. Soc., v. 33, p. 456 (not. biol.) (1929). - D. l. l., id., l. c., v. 34, p. 1009 (not. biol.) (1931). forma significans Stich. $D$. l. s., $\mathrm{H}$. Stichel, Ins.-Börse, v. 18, p. 317 (1901) Id., Berlin. Ent. Zeit., Sitz.-Ber., p. (11) (1902). - D. l. ab .s., id., Deutsch. Ent. Zeit. Iris, v. 15, p. 82,83 (1902). - D. l., forma s., id., Gen. Ins., fase. 31, p. 9 (1905). - D. l. s. "Rogenzeitform", Fruhstorfer: A. Seitz, Großschmett. Erde, v. 9, p. 445 (1911). - D. $l$. , ,wot season form" s., W. H. Evans, Journ. Bombay Nat. Hist. Soc., v. 22, 1913, p. 763 (1914).

D. le pida l. (in synon.), W. H. Evans, ut antea (1923 \& 27).

subsp. ceylonica Fruhst. D. $l$. c.. Fruh- Ceylon storfer: A. Seitz, Großsehmett. Erde, v. 9, p. 445, t. $106 \mathrm{~d}$ (lepida o7, corr. p. 1135) (1911 \& 27). - D. l. "race" c., W. H. Evans, Journ. Bombay Nat. Hist. Soc., v. 22,1913 , p. 763 (1914). - D. l. c., id., l. c., v. 29 , p. 797 (1923). - Id., Identif. Ind. Butterfl., p. 87 (1927).

D. le pida, F. Moore, Lop. Ceylon, v. 1, p. 36 (cit. excopt.), t. 18 , f. 1, 1 a, b (1880). Nicéville \& Manders, Jouru. Asiat. Soc. Bengal, v. 68. II, p. 186 (1899). - Manders, Journ. Bombay Nat. Hist. Soc., v. 16, p. 78 (1904). - Fruhstorfer: A. Seitz, Großschmett. Erde, v. 9, t. $106 \mathrm{~d}$, (corr., p. 1135, vide supra) (1911). - Ormiston, Spol. Zeylan., v. 11, p. 17 (not. biol.) (1918).

D. le $p$ ida (part.), ut antea: F. Moore (1880, 1895); Mar'shall \& Nicéville (1883); Staudinger (1887.); Fruhstorfer (1900); Stichel (1902, 1905); Bingham (1905); Bell (1909); Evans (1912).

\section{Sectio Sfenopoecili}

H. Stichel, Gen. Ins., fasc. 31, p. 9 (1905).

\section{Cohors a. Sondaiciformes}

H. Stichel, Gen. Ins., fasc. 31, p. 9 (1905).

- sondaica Boisd. D. s., Boisduval, Spec. Gén. Iuép., v. 1, Expl. Planchos, p. 4 (1836). - H. Stichel, Gen. Ins., fasc. 31, p. 9 (1905).

tullia (Cram.) "Tullia“, Papilio t. (nom. praeocc.: $P$. tullia O. F. Müller, 1764), P. Cramer, Pap. Exot., p. 127, 154 (1775 \& 1776). - Discophora t., H. Stichel, Deutsch. Ent. Zeit. Tris, v. 15, p. 65, 67, 85; p. 61, f. 1 ; p. 62 , t. 1 , f. 9,9 a $\rightarrow$ (organ. copul.)

Typ.:

Karwar 
(1902). - Shelford, Journ. Straits Branch Asiat. Soc., Nr. 41, p. 108 (1904). - Fruhstorfer: A. Seitz, Großschmett. Erde, v. 9, p. 442 (1911).

forma despoliata Stich. D. tullia sondaica Typ.:

ab. d., H. Stichel, Deutsch. Ent. Zeit. Iris, Malakka v. 15, p. $85,91(1902)$ - D. s. s. forma $d$., id., Gen. Ins., fasc. 31, p. 9 (1905). $-D$. tullia (d.), Fruhstorfer: A. Seitz, Großschmett. Erde, v. 9, p. 443 (1911). - D. t. d., W. H. Evans, Journ. Bombay Nat. Hist. Soc., v. 22, 1913, p. 763 (1914). (Forma communis).

D. tullia (non Papilio t. Cramer), Distant, Rhop. Malay., p. 74 (part.), t. 7, f. 8; D. $t$. var., p. 426 (1883 \& 86).

D. zal (non Westwood), G. F. I. Marshall \& Nicéville, Butt. Ind. Burm. Ceyl., v. 1, p. 299 (part.) (1883). - D. tullia z., W. H. Evans, Journ. Bombay Nat. Hist., v. 29, p. 796 (in synon.) (1923). - Id., Identif. Butterfl., p. 87 (in symon.) (1927).

subsp. sondaica Boisd. D. 8., Boisduval, Java, Spec. Gén. Lép., v. 1, Expl. Planches, p. 4, Bali t. 12 , f. 3 (1836). - (E. Doubleday \&) Westwood, Gen. Diurn. Lep., v. 2, p. 331 (1851). - Herrich-Schäffor, Corr.-Bl. Zool.Min. Ver. Regensb., v. 19, p. 88 (1865). W. F. Kirby, Cat. Diurn. Iep., p. 117 (1871). - Distant, Rhop. Malay.s App., p. 426 (part.) (1886). - A. Pagenstecher, Jahrb. Nassau. Ver. Naturk., v. 43, p. 96 (1890). - F. Moore, Lep. Ind., v. 2, p. 198 (1895). - Fruhstorfer, Berlin. Ent. Zeit., v. 41,1896 , p. $301(1897)$. - Nicéville \& Elwes, Journ. Asiat. Soc. Bengal, v. 66. II, p. 682 (1898). - D. tu.77ia 8., Fruhstorfer, Berlin. Ent. Zeit., v. 45, p. 13 (1900). H. Stichel, Doutsch. Ent. Zeit. Iris, v. 15, p. 85,89 (cit., distr. part.) t. 1, f. $9 \mathrm{c}, \mathrm{d}$ (organ. copul.) (1902). - D. sondaica 8., id., Gen. Ins., fasc. 31, p. 9 (part.) (1905). - D. tullia 8., Fruhstorfer: A. Seitz, Großschmett. Ende, v. 9, p. 444, t. 106 a $\left(\sigma^{\prime}\right), 106 \mathrm{~b}$ () (1911).

subsp. symphronia Fruhst. D. tullia 8., Borneo, Fruhstorfer: A. Seitz, Großsohmett. Erde, Sumatra, v. 9, p. 444 (1911).

D. tullia (non Papilio t., Cramer), Herb. Druce, Proc. Zool. Soc. London, p. 341 (1873). - A. G. Butler, Trans. Linn. Soc. London, ser. 2, Zool., v. 1, p. 538 (1879). -

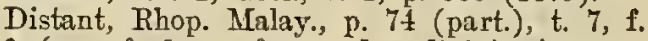
9 (non f. 8: = forma despoliata); App., p. 426 (distr. part.) (1882). - Id., Ánn. Mag. Nat. Hist., ser. 5, v. 12 , p. 352 (1883). B. Hagen, Tijdschr. Nederl. Aardr. Gen., sor. 2, v. 7, p. 200 (1890).

Sunda-

Inseln, Philippinen

Malakka 
D. sondaica (non Boisduval), Distant, Rhopal. Malay., App., p. 426 (part.), t. 43, f. 7 (1886). - O. Staudinger (\& Schatz), Exot. Schmett., v. 1, p. 190 (1887). - B. Hagen, Tijdschr. Nederl. Aardr. Gen., ser. 2, v. 7, p. 199 (1890). - L. Martin, Deutsch. Ent. Zeit. Lep., v. 8, 1895, p. 263 (1896). - Nicéville \& L. Martin, Journ. Asiat. Soc. Bengal, v. 61. II, 1895 , p. 396 (1896). A. Pagenstecher, Abh. Senckenb. Naturf. Ges., v. 23, p. 405 (distr. part.) (1897). - D. tullia s., Shelford, Journ. Straits Branch Asiat. Soc., Nr. 41, p. 109 (1904). - D. s., Mloulton, 1. c., Nr. 65, p. 18 (distr. part.) (1913).

subsp. tul liana Stich. :D. 8. tulliana, H. Süd-

Stichel, Gen. Ins., fasc. 31, p. 10 (1905).

tullia (Cramer) "Tullia", Papilio t. (nom. China, praeocc.: Papilio t. O. F. Müller, 1764), P. Cramer, Pap. Exot., v. 1, p. 127, t. 81 , f. A, B; p. 154 (1775\& 76). - P. (Nymphalis) t., Gooze, Entom. Beytr., v. 3. I, p. 289 (1779). - P. t., J. C. Fabricius, Spoc. Ins., p. 76 (1781). - C. Stoll, Essai Syst. Lop.: P. Cramer, Top. Exot., v. 4, App., p. 8 (1782). - P. (Nymphalis Gemmatus) t., [D. H. Schnoidor], Nomenal. Entom., p. 36 (1785). - P. t., J. C. Fabricius, Mant. Ins., v. 2, p. 38 (1787). - P. (Nymphalis) t., Gmelin: Linné, Syst. Nat., ed. 13, v. 1. V, p. 2303 (1790). - P. t., Jung', Alphab. Verz. Schmett., v. 2, p. 251 (1792). - J. C. Fabricius, Ent. Syst., v. 3. I, p. 98 (1793). - P. tullius, (Jablonsky \&) Herbst, Naturs. Ins., Schmett., v. 8, p. 239 , t. 220 , f. 3,4 (9) (1796). - P. tullia, Turton, Gen. Syst. Nat. Linné, v. 3. II, p. 94 (1806). - Moera t., Jac. Hübner, Verz. Schmett., p. 51 [1818]. - Morpho t., (Latreille \&) Godart, Enc. Meth., v. 9, p. 446 (1824). - Verloren, Cat. Ins. Iep. Cramer, v. 1, p. 42; v. 2, p. 201 (1837). - Discophora t., (E. Doubleday \&) Westwood, Gen. Diurn. Lep., v. 2, p. 331 (1851). - Chenu \& H. Lucas, Enc. Hist. Nat. Papill., p. 163 (1851). - Ménétriés, Enum. Corp. Anim. Mus. Petropol., Lep. Suppl., v. 1, p. 77; v. 2, p. 121 (1857). - Herrich-Schäffer, Corr.-Bl. Zool.-Min. Ver. Regensb., v. 19, p. $88(1865)$. - W. F. Kirby, Cat. Diurn. Iep., p. 116 (cit. part.) (1871). - F. Moore, Proc. Zool. Soc. London, p. 826 (1878). - O. Staudinger '(\& Schatz), Exot. Schmett., v. 1, p. 189 (part.) (non t. $63:=D$. tulliana zal forma indica) (1887). - F. Moore, Lep. Ind., v. 2, p. 107 (1895). - J. J. Walker, Trans. Ent. Soc. London, p. 442, 449 (not. biol.) (1895). Eimer (\& Fickert), Entstehg. d. Arten, pars 2, Orthogen., p. 180, 235, 236 (1897). - 
Fruhstorfer, Berlin. Ent. Zeit., v. 45, p. 13 (1900). - Nicéville, Journ. Asiat. Soc. Bengal, v. 71. II, p. 8 (larva) (1902). - D. $t$. (typica), H. Stichel, Deutsch. Ent. Zeit. Iris, v. 15 , p. 86 ; p. 61 (organ. copul.) (1902). - Shelford, Journ. Straits Branch Asiat. Soc., Nr. 41, p. 108 (part.) (1904). Bell, Journ. Bombay Nat. Hist. Soc., v. 19, p. 467,473 (Biol.) (1909). - D. t. + D. $t$. tulliana, Fruhstorfer: A. Seitz, Großschmett. Erde, v. 9, p. 443, t. 106 c (o', 우) (1911). D. tullia, W. H. Evans, Journ. Bombay Nat. Hist. Soc., v. 22, 1913, p. 763 (1914).

? D. celinde (non Papilio c. Stoll), Eastlake, Proc. Ac. Nat. Sci. Philadelphia, p. 86 (1885).

subsp. $h a i n a n e n s i s$ Fruhst. D. tullia $h$., Hainan

Fruhstorfer: A. Seitz, Großschmett. Erde, v. 9 p. 443 (1911). - Joicey \& Talbot, Bull. Hill Mus., จ. 2, p. 184 (1928).

D. tull ia, Crowley, Proc. Zool. Soc. London, p. $505(1900)$.

subsp. zal Westw. $\left.{ }^{1}\right)$ D. z., (E. Doubleday \&) SikkimWestwood, Gen. Diurn. Lop., v. 2, p. 331 (no- Burma, ta) (Q) (1851). - Herrich-Schäffer, Corr.- Siam, B1. Zool-Min. Ver. 'Regensb., v. 19, p. 88 (1865). - Westwood, Trans. Ent. Soc. Iondon, n. ser., $\nabla .4,1857$, p. 188, t. $21, f .5$, 6 (Q) (1858). - F. Moore, Proc. Zool. Soc. London, p. 761 (1865). - W. F. Kirby, Cat. Diurn. Lep., App., p. 646 (1871). - F. Moore, Proc. Zool. Soc. London, p. 826 (1878). - W. F. Kirby, Sci. Proc. R. Dublin Soc., ser. 2, v. 2, p. 300 (1880). - Distant, Rhop. Malay., p. 74 (part.), p. 426 (1882 \& 86). - Id., Ann. Mag. Nat. Hist., ser. 5, v. 12 , p. 353 (1883) - G. F. L. Marshall \& Nicéville, Butt. Ind. Burm. Ceryl., v. 1, p. 299 (Q) (1883). - Nicéville, Journ. Asiat. Soc. Bengal., v. 54. II, p. 43 (1885). O. Staudinger (\& Schatz), Exot. Schmett., v. 1, p. 190 (D. sondaica "Lokalform" aut aberr.) (1887) - G. Semper: O. Semper, Reis. Arch. Philippin., pars 2. V, Schmett., v. 1, p. 73 (in eit.) (1887). - Elwes \& Nicéville, Journ. Asiat. Soc. Bengal, v. 55. II, 1886, p. 419 (1887). - F. Moore, Lop. Ind., v. 2, p. 191, t. 152, fig. 1, 1 a (O'), fig. $1 \mathrm{~b}$, c (Q) (1895). - Fruhstorfer, Berlin. Ent. Zeit., v. 45, p. 14 (1900). D. tullia ab. z., H. Stichel, Deutsch. Ent. Ent. Zeit. Iris, v. 15, p. 85, 91 (? forma tempest.); t. 1, f. 8 a, b (organ. copul.) (1902). - D. t. var. z., Bingham, Faun. Brit. Ind., Butterfl., v. 1, p. 203 (1905). $D$. sondaica indica forma $z$., H. Stichel,

Tenasserim ? Südost-

Tibet

1) Die Zitate gründen sich zumeist auf die FundortAngaben, ohne Gewähr für zutreffende Beziehung. 
Gen. Ins., fasc. 31 , p. 10 (1905). - $D$. tullia z., Fruhstorfer: A. Seitz, Großschmett., Erde, ఛ. 9, p. 443 (Form der trockenen Periode) (1911). - D. z., W. H. Evans (sec. Fruhstorfer, 1911), Journ. Bombay Nat. Hist. Soc., v. 22,1913, p. 763 (1914). - D. tullia z., id., l. c. v. 23, p. 538 (1915). — Godfrey, Journ. Nat. Hist. Soc. Siam. v. 2, p. 124 (1916). - W. H. Evans, Journ. Bombay Nat. Hist. Sac., v. 29, p. 796 (sine synon.), t. 16. fig. E 10. 1 (Q) (1923). - Id., Identif. Ind. Butterfl., p. 87, etc. ut antea (1927). - Godfrey, Journ. Siam. Soo. Nat. Hist. Suppl., v. 7, p. 122 (1927). - Id., I. c., p. $262(1930)$.

? D. $t$ u $l l i a$ (non Papilio $t$. Cramer) $)^{1}$, E. Doubleday, List Irep. Brit. Mus., pars 1, p. 144 (1844). - Horsfield \& F. Moore, Cat. Lep. Mus, E.-Ind. Comp., v. 1, p. 211, t. 12 , f. 15,15 a (larva, chrysal.) (1857). F. Moore, Proc. Zool. Soc. London, p. 761 (1865). - A. G. Butler, Cat. Diurn. Lep. Fabr., p. 45 (1869). - Id, Proc. Zool. Soc. London, p. 605 (1881). - Distant, Rhop. Malay., p. 74 (part.) (1882). - Nicéville, Journ. Asiat. Soc. Bengal, v. 51. II, p. 57 (1882). - G. F. L. Marshall \& Nicéville, Butt. Ind. Burm. Ceyl., v. 1, II, p. 298, fig. ণ", $\supsetneq$ (1883). - Nicévillo, Journ. Asiat. Soc. Bengal, v. 54. IT, p. 43 (1885). - Wilh. Müller, Zool. Jahrb., v. 1, p. 611 (larva, chrysal. sec. Moore, 1857). - Wood-Mason \& Nicéville, Journ. As. Soc. Beng., v. 55. II, 1886, p. 353 (cit. excopt.) (1887). - Elwes \& Nicéville, 1. c., v. 55. II, 1886 , p. 419 (1887). - E. Y. Watson, Journ. Bombay Nat. Hist. Soc., v. 3, p. 20 (1888). - D. $t$. var., Elwes (\& Möller), Trans. Ent. Soc. London, p. 281, 331 (1888). - D. t., Manders, I. c., p. 519 (larva, chrysal.) (1890). - Swinhoe, l. c., p. 275 (1893). - Bingham, Faun. Brit. Ind,, Butterfl., v. 1, p. 202 (cit. part.) (1905). - Tytler, Joum. Bombay Nat. Hist. Soc., v. 21, p. 55 (1911). - Antram, Butterfl. Ind., p. 24, f. 254 (1924).

D. celinde (non Papilio c. Stoll), W. F. Kirby, Cat. Diurn. Lep., p. 116 (in cit.) (1871).

? D. sondiaca (!) (non D. sondaica Boisduval), Elwes (\& Möller), Trans. Ent. Soc. Iondon, p. 281 (1888).

forma indica Stauding. D. tullia var. i., O. Typ.:

Staudinger (\& Schatz), Exot. Schmott., v. 1, Indien t. 63 (ㅇ), p. 190 (part.) (1886 \& 87). D. $t$. var. $i .$, Robbe, Ann. Soc. Ent. Belg., v. $36, \mathrm{p} .128(1892)$. - D. t., D. $t$. var. $i ., \mathrm{Ni}-$

1) Siehe Fußnote p. 154. 
céville, Gazetteer Sikhim, List Butterfl., p. 130 (1894). - D. i., F. Moore, Lep. Ind., v. 2 , p. 153 , t. 153, f. $1 \mathrm{a}, \mathrm{b}\left(\mathrm{o}^{*}\right), 1 \mathrm{c}, \mathrm{d}(\mathrm{O})$ (1895). - D. tullia i., Fruhstorfer, Berlin. Ent. Zeit., v. 45, p. 13 (part.) (1900).H. Stichel, Deutsch. Ent. Zeit. Iris, v. 15, p. 86,87 (cit. part.) (1902). - Id., Gen. Ins., fasc. 31, p. 10 (cit. part.) (1905). Fruhstorfer: A. Seitz, Großschmett. Erde, v. 9, p. 443 (Genemt. der Regenzeit) (1911). - D. t. „raos" i., W. H. Evans, Journ. Bombay Nat. Hist. Soc., v. 21, p. 751 (part.) (1912). - D. i., id., 1. c., v. 22, 1913, p. 763 (D. tullia wet scason form) (1914). - D.t. i., Ellis, l. c., v. 25, p. 107 (1917).

- Ollenbach, 1. c., v. 27, p. 888 (1921). D. tullia zal var. i., W. H. Evans, l. c., v. 29, p. 796 (1923). - Id., Identif. Ind. Butterfl., p. 87 (1927).

forma s pi lo p te ra Nicéville \& Möller D. s., Typ.:

Nicéville \& Möller: Elwes \& O. Möller, Sikkim

Trans. Ent. Soc. London, p. 281, 331 (1883).

- ? E. Y. Watson, Journ. Bombay Nat. Hist. Soc., v. 5, p. 36 ( $\sigma^{x} \sigma^{x}$ not typical) (1891). - Nicéville, Gazetteer Sikhim, List Butterfl., p. 130 (? dry season of tullia) (1894). - F. Moore, Lep. Ind., v. 2, p. 195, t. 154 , f. 1 ( ( $\left.\sigma^{\prime}\right)$, f. 1 a (Q) $(1895)$. - E. Y. Watson, Journ. Bombay Nat. Hist. Soc., v. 10, p. 652 (? D. cheops, D. tullia) (1897). - D. zal s., Fruhstorfer, Berlin. Ent. Zeit., v. 45, p. $14(1900)$. - D. tullia ab s., H. Stichel, Deutsch. Ent. Zoit. Iris, v. 15, p. 85, 93 (1902). - D. sondaica indica forma s., id., Gen. Ins., fase. 3l, p. 10 (1905). D. tullia var. 8., Bingham, Faun. Brit. Ind., Butterfl., v. 1, p. 203 (1905). - D. tullia s., Fruhstorfer: A. Seitz, GroBschmett. Erdo, v. 9, p. 443 (Fehlfärbung) (1911). - D. tullia zal var. s., W. H. Evans, Journ. Bombay Nat. Hist. Soc., v. 29, p. 797 (1927). Id., Identif. Ind. Butterfl., p. 87 (1927).

subsp. mus cina Stich. D. m., H. Stichel, VorderIns.-Börse, v. 29, p. 125 (1902). $-D$. Indien tullia $m_{\text {., }}$ id., Deutsch. Ent. Zeit. Iris, v. (Karwar, 15, p. 85,88 , t. 2 , f. 2 ; t. 1, f. 9a (organ. Kanara) copul.) (1902). - D. sondaica m., H. Stichel, Gen. Ins., fase. 31, p. 10 (1905). D. tullia m., Fruhstorfer: A. Seitz, Großschmett. Erde, v. 9, p. 443 (1911). - D. $t$. "Nace" $m$., W. H. Evans, Journ. Bombay Nat. Hist. Soc., v. 22, 1913, p. 763 ,(1914). - Id., 1. c., v. 29, p. 796 (1923). - Id., Identif. Butterfl., p. 67 (1927).

$D$. indica (non D. tullia var. $i$. Staudinger), Nicéville, Journ. Asiat. Soc. Bengal, v. 69. II, p. 220 (1900). - D. tullia "race" $i$., E. Y. Watson, Journ. Bombay Nat. Hist. Soc., v. 21, p. 751 (part.) (1912). 
subsp. Semperi D. s., F. Moore, Lep. Ind., Philippinen v. 2 , p. 198 (1895). - D. tullia 8., Fruh- (Mindanao, storfer, Berlin. Ent. Zeit., v. 45, p. 13 (1900). Sibulan)

- H. Stichel, Deutsch. Ent. Zeit. Iris, v. 15, p. 86,91 (1902). - D. sondaica s., Gen. Ins., fasc. 31, p. 10 (1905). - D. tullia s., Fruhstorfer: A. Seitz, Großschmett. Erde, v. 9, p. 443 (1911).

D. zal (non Westwood), G. Semper: O. Semper, Reis. Arch. Philippin., pars 2. V, Schmett., v. 1, p. 73 (cit. except.), t. 13, f. $10,11\left(\sigma^{*}\right)$; p. 333 (1887 \& 92). - Elera, Cat. Faun. Filipin., v. 2, p. 271 (cit. except.) (1895).

\section{Cohors b. Simpliciformes}

H. Stichel, Gen. Ins., fasc. 31, p. 10 (1905).

simplex Stauding. D. s., O. Staudinger, Deutsch. Palawan

Ent. Zoit. Lep., v. 2, p. 42 (1889). - G. (= Paragua)

Semper: C. Semper, Reis. Arch. Philippin., pars 2. V, Schmett., v. 1, p. 333 (1892). F. Moore, Lep. Ind., v. 2, p. 198 (1895). Elera, Cat. Faun. Filipin., v. 2, p. 271 (1895). - Frubstorfer, Berlin. Bnt, Zeit., v. 45 , p. $14(1900)$, - H. Stichel, l. c., v. 46, Sitz.-Ber., p. (4) (1901). - H. Stichel, Deutsch. Bnt. Zeit. Iris, v. 15, p. 65, 67, 93; p. 62 , t. 1, f. 10 (organ. copul.) (1902). Id., Gen. Ins., fasc. 31, p. 10 (1905). D. 8. 8., Fruhstorfer: A. Seitz, Großschmett. Erde, v. 9, p. 445 , t. 106 c (1911).

amethystina Stich, D. a., H. Stichel, Ins.-Börse, v. Borneo

17, p. 69 (1900). - Id,, Berlin. Ent. Zeit., v. 46, Sitz.-Ber., p. (4) (1901). - Id., Deutsch. Ent. Zeit. Iris, v. 15, p. 65, 94, t. 2 , f. 1 ; p. 62, t. 1 , f. 11 (organ. copul.): (1902). - Shelford, Journ. Straits Branch Asiat. Soc., Nr. 41, p. 109 (1904). - H. Stichel, Gen. Ins., fasc. 31, p. 11 (1905). D. simplex a., Fruhstorfer: A. Seitz, Großschmett. Erde, v. 9, p. 445 (1911). D. a., Moulton, Journ. Straits Branch Asiat. 11 Soc., Nr. 65, p. 18 (1913).

\section{Genus Enispe Westw.}

E. Doubleday (\& IVestwood), Gen. Diurn. Lep., t. 40 (1848); Westwood, v. 2, p. 292 (1850). - Chenu \& H. Lucas, Enc. Hist. Nat., Papill., p. 142 (1851). - Horsfield \& F. Moore, Cat. Lep. Ins. Mus. E.-Ind. Comp., v. 1, p. 212 (1857). - Herrich-Schüffer, Corr.Bl. Zool-Min. Ver. Regensb., v. 18 , p. 112 ; v. 19 , p. 88 (1864 \& 65). - W. F. Kirby, Cat. Diurn. Lep., p. 117 (1871). - S. H. Scudder, Proc. Amer. Ac. Sci., v. 10, p. 163 (typ.: enthymius, error. typogr.) (1875). - W. F. Kirby, Entomologist, v. 10, p. 292 (1877). - Id., Sci. Proc. R. Dublin Soc., ser. 2, v. 2, p. 300 (1880). - G. F. L. Marshall \& Nicéville, Butt. Ind. Burm. Ceyl., v. 1, p. 283, 299 (1883). - B. Gerhard, Berlin. Ent. Zreit., v. 27, p. 181 (distr. 
geogr.) (1883). - O. Staudinger (\& Schatz), Exot. Schmett., v. 1, p. 190 (1887). - L. Glaser, Cat. Etymol. Col. Lep., p. 288 (spec. omiss.) (1887). - E. Haase, Corr.-Bl. Ent. Ver. Iris, v. 1, p. 310 (organ. odor.) (1888). - Röber: O. Staudinger \& Schatz, Exot. Schmett., v. 2, t. 31 (Morphol.), p. 184, 187 (1888 \& 89). B. Hagen, Tijdschr. Nederl. Aardr. Gen., ser. 2, v. 7, p. $200(1890)$. Leech, Butt. Chin. Jap. Cor., v. 1, p. 110 (1892). - Swinhoe, Trans. Ent. Soc. London, p. 276 (1893). - W. F. Kirby, Handb. Lop. (Allens Natural. Libr.), v. 1, p. 198 (1894). - F. Moore, Iep. Ind., v. 2, p. 198 (1895). - L. Martin, Deutsch. Ent. Zeit. Lep., v. 8, p. 263 (1896). - H. Stichel, Deutsch. Ent. Zeit. Iris, v. 15, p. 59 (1902). - L. C. H. Young, Journ. Bombay Nat. Hist. Soc., v. 15, 1903, p. 298, 486 (1903 \& 04). - Shelford, Journ. Straits Branch Asiat. Soc., Nr. 41, p. 109 (1904). - Bingham, Faun. Brit. Ind., Butterfl., v. 1, p. 182, 197 (typ.: E. euthymius) (1905). - H. Stichel, Gen. Ius., fasc. 31, p. 11, t., fig. 2 (Morphol.) (1905). Id.: A. Seitz, Großschmett. Erdo, v. 1, p. 157 (1908). - Fruhstorfer: l. c., v. 9, p. 448 (1911). - W. H. Evans, Journ. Bombay! Natis Hist. Soc., v. 21, p. 572 (1912). - MIoulton, Journ. Straits Branch Asiat. Soc., Nr. 65, p. 18 (1913). - W. H. Evans, Journ. Bombay Nat. Hist. Soc., v. 29, p. 793, 797, t. 16, E 11 (1923). - Antram, Butterfl. Ind., p. 122 (1924). - Lo Cerf, Enc. Ent., ser. B 3, Lep., v. 1, p. 141 (Morphol.) (1926). - W. H. Evans, Identif. Ind. Butterfl., p. 84, 87, t. 16, E 11 (1927).

Adolias, E. Doubleday, Ann. Mag. Nat. Hist., v. 16, p. 179 (1845). - Id., List Lep. Brit. Mus., App., p. 27 (1848). - N. D. Riley, Cat. Type Specim. Rhop. Brit. Mus., pars 1, p. 20 (1924).

euthymius (Doubl.) Adolias e., E. Doubleday, Ann. NepalMag. Nat. Hist., v. 16, p. 179 (1845). - Assam, Enispe e., H. Stichel, Gen. Ins., fasc. 31, p. Burma, 12 (1905). - Fruhstorfer: A. Seitz, Groß- Siam, schmett. Erde, v. 9, p. 448 (1911).

Malayische

Halbinsel,

Sumatra,

Borneo

- subsp. euthymius (Doubl.) Adolias e., E. Nepal, Doubleday, ut antea (1845). - Id., List Lep. Sikkim, Brit. Mus., App., p. 27 (1848). - Enispe e., Assam, E. Doubleday (\& Westwood), Gen. Diurn. SüdostLep., t. 40, f. 2 (1848); Westwood, v. 2, p. Tibet 292 (1850). - Chenu \& H. Lucas, Enc. Hist. Nat. Papill., p. 142, f. 259 (1851). Herrich-Schäffer, Corr.-Bl. Zool.-Min. Ver. Regensb., v. 19, p. 88 (1865). - F. Moore, Proc. Zool. Soc. London, p. 761 (1865). Horsfield \& F. Moore, Cat. Lep. Ins., E.-Ind. Comp., v. 1, p. 212 (1857). - W. F. Kirby, Cat. Diurn. Lep., p. 117 (1871). - Id., Sci. Proc. R. Dublin Soc., ser. 2, v. 2, p. 300 (1880). - G. F. L. Marshall \& Nicéville, Butt. Ind. Burm. Ceyl., v. 1. II, p. 300, fig. ( $\left.\sigma^{7}\right)(1883)$. - O. Staudinger (\& Schatz), Exot. Schmett.; v. 1, t. 63, p. 190 (1886 \& 87). - Wood-Mason \& Nicéville, Journ. Asiat. Soc. Bengal, v. 55. II, 1886, p. 353 (part.) (non t. 15, f. 1: = forma tesselata) (1887). - Elwes (\& O. Möller), Trans. Ent. 
Sac. London, p. 281, 332 (1888). - Nicéville, Journ. Bombay Nat. Hist. Soc., v. 5, p. 296 (1890). - Swinhoe, Trans. Fnt. Soc. London, p. 276 (1893). - Nicéville, Gazetteer Sikhim, List Butterfl., p. 130 (1894). F. Moore, Lep. Ind., v. 2, p. 198, t. 155, f. 1, $1 \mathrm{a}\left(\sigma^{7}\right), 1 \mathrm{~b}, \mathrm{c}($ ( ) $)(1895)$. - Eimer (\& Fickert), Entstehg. d. Arten, pars 2, Orthogen., p. 235, 346 (1897). - E. Y. Watson, Journ. Bombay Nat. Hist. Soc., v. 10, p. 652 (1897). - Id., 1. c., v. 15,1903, p. 485 (1904). - Bingham, Funn. Brit. Ind., Butterfl., v. 1, p. 198 , t. 4 , f. 31 (1905). $-E$. euthymius e., H. Stichel, Gen. Ins., fasc. 31, p. 12 (1905). - Fruhstorfer: A. Seitz, Großschmett. Erde, v. 9, p. 448 (sine synon.) (1911). - W. H. Bvans, Journ. Bombay Nat. Hist. Soc., v. 21, p. 572 (1912). - Tytler, 1. c., v. 23, p. 228 (1914). - W. H. Evans, 1. c., v. 23, p. 538 (1915). - E. V. Ellis, 1. c., v. 25, 107 (1917). - Ollenbach, 1. c., v. 27 , p. 888 (1921). - W. H. Evans, 1. c., v. 29, p. 797 (sine synon.) (1923). - Antram, Butterfl. Ind., p. 122, fig. 250 (1924). - Adolias e., N. D. Riley \& Gabriel, Cat. Type Specim. Rhop. Brit. Mus., pars 1, p. 20 (1924). - Enispe e., W. H. Evans, Identif. Ind. Butterfl., p. 87 (1927). forma tess elata Moore E. t., F. Noore, Proc. Zool. Soc. London, p. 521 (1883). Id., Lep. Ind., v. 2, p. 200, t. 156, f. 1, 1 a $\left(0^{\prime}\right), 1 \mathrm{~b}, \mathrm{c}(q)(1895)$. - Nicéville \& L. Martin, Journ. Asiat. Soc. Bengal, v. 64. II, 1895 , p. 396 (spec. distinct. nulla) (1896). $E$. e uthymius var. (ot ab.) t., O. Staudinger, Deutsch. Fint. Zeit. Lep., ४. 9, 1896, p. $232(=$ silhetensis) (1897). - E. e. tesselatus, II. Stichel, Gon. Ins., fasc. 31, p. 12 (cit. part.) (1905). - E. e. tesselata, Fruhstorfer: A. Seitz, Großschinett. Ende, v. 9, p. 448 (corrig: p. 449, 1111: tesselatus) (1911 \& 27). - E. enthymius (!) var. tesselata, Tytler, Journ. Bombay Nat. Hist. Soc., v. 21, p. 54 (1911). $-E$. e. "season. form" " $t$., W. H. Evans, 1. c., v. 21, p. 572 (1912). E. e. 't., Moulton, Journ. Straits Branch Asiat. Soc., Nr. 65, p. 18 (1913). - E. e. var. $t$., Tytler, Journ. Bombay Nat. Hist. Soc., v. 23, p. 228,229 (in dry and wet season) (1914). - E. e. "form" t., E. V. Ellis, 1. c., v. 25, p. 107 (dry season) (1917). - E. e. var. t., W. H. Evans, 1. c., v. 29 , p. 797 , t. $16, \mathrm{E} 11.2$ (1923). - E. t., N. D. Riley \& Gabriel, Cat. Type Specim. Rhop. Brit. Mus., pars 1, p. 57 (1924). - E. e. var. t., W. H. Evans, Identif. Ind. Butterfl., p. 87, t. 16. E 11. 2 (1927). - E. e. e. var. t., Godfrey, Journ. Siam Soc. Nat. Hist., Suppl., v. 7, p. 263 (part.) (1930).

'Typ.:

Darjeeling, Nepal 
sylhetensis Stauding. E. e. var. s., O. Staudinger (\& Schatz), Exot. Schmett., v. 1, p. 191 (1887).

E. e uthymius (non Adolias e. Doubleday), Wood-Mason \& Nicéville, Journ. Bombay Nat. Hist. Soc., p. 353 (part.), t. 15, f. 1 (1886). - W. II. Evans, 1. c., v. 29, p. 797 (in cit. $E$. silhetensis) (1923). - Id., Identif. Butterfl., p. 87 (ut antea) (1927).

forma melaena Tytler E. e. „form" (var.) Typ.: m., Tytler, Journ. Bombay Nat. Hist. Soc,, Manipur v. 23, p. 228,229 (in dry and wet season), (1914).

E. $e u t h y m i u s$, W. H. Evans, ut antea (1923, 1927).

subsp. intermedia Rothsch. E. e. i., W. BurmaRothschild, Nov. Zool., v. 23, p. 315 (1916). Malayische - E.e. „Pace" i. W. H. Evans, Journ. Bom- Halbinsel, bay Nat. Hist. Soc., v. 27, p. 90 (1920).

E. e $u t h y m i u s$, Elwes, Proc. Zool. Soc. London, p. 271 (1891). - W. H. Evans, Journ. Bombay Nat. Hist. Soc, v. 29, p. 797 (in synon.) (1923). - Id, Identif. Ind. Butterfl., p. 87 (ut antea) (1927).

$E$. e. forma tessel $u t u$, Goxlfrey, Journ. Siam Soc. Nat. Hist. Suppl., v. 7, p. 263 (part.) (1930).

subsp. duranius Fruhst. E. e. d., Frub- Sumatra storfer: A. Seitz, Großschmett. Erde, v. 9, p. 448 , t. $105 \mathrm{c}(1911)$. - E. e. ,race" durania, W. H. Evans, Journ. Bombay Nat. Hist. Soc., v. 27, p. 90 (1920).

E. e $u$ ty $\mathrm{m}$ ius (!), B. Hagen, Tijdschr. Nederl. Aardr. Gen., ser. 2, v. 7, p. 199 (1890). - E. euthymius, L. Martin, Deutsch. Ent. Zeit. Lep., v. 8, 1895, p. 264 (1896). Nicéville \& L. Martin, Journ. Asiat. Soc. Bengal, v. 64. II, 1895, p. 396 (1896).

subsp. $m i l v u s$ Stauding. $E . m$., O. Stau- Borneo dinger, Deutsch. Ent. Zeit. Lep., v. 9, p. 231, t. 5, f. 4 (milous, error typ.) (1897). - Shelford, Journ. Straits Branch Asiat. Soc., Nr. 41, p. 109 (1904). - E. euthymius milvus, H. Stichel, Gen. Ins., fasc. 31 , p. 12 (1905). - Iruhstorfer: A. Seitz, Großschmett. Erde, v. 9, p. 448, (1911). - Noulton, Journ. Straits Branch Asiat. Soc., Nr. 65 , p. 18 (1913).

Iunata Leech E. lunatus, Leech, Entomologist, v. Süd24, Suppl., p. 26 (1891). - Id., Butterfl. China, Chin. Jap. Cor., v. 1, p. 111, t. 1, f. 1 Tibot, (Ј), 2 (Q) (1892). - Nicéville, Gazetteer Hainan Sikhim, List Butterfl., p. 130 (1894). - H. Stichel, Gen. Ins., fasc. 31, p. 12 (1905). Id.: A. Seitz, Großschmett. Erde, v. 1, p. 157, t. $49 \mathrm{~b}$ (1908). - Fruhstorfer: 1. c., v. 9 , p. 449 (1911). - N. D. Riley \& Ga- 
briel, Cat. Type Specim. Rhopal. Brit. Mus., pars 1, p. 32 (1921). - Joicey \& Talbot, Bull. Hill Mus., v. 2, p. 184 (1928).

forma enervatu Stich. E. lunatus forma Typ.: e., H. Stichel, Gen. Ins., fasc. 31, p. 12, t., Siaolu, fig. 6 (P) (1905). - E. $l$. "Form" $l$., id.: Ta-tsien-lu A. Seitz, Großschmett. Erde, v. 1, p. 157 (1908). - E. l. e., Fruhstorfer: 1. c., v. 9, p. 449 (Trockenzeitform) (1911). - E. $i$. forma c., Draeseke, Deutsch. Ent. Zeit. Iris, v. 39, p. 57 (1925).

cyenus Westw. E. c., (It. Doubleday \&) Westwood, NordGen. Diurn. Lop., v. 2, p. 330 (1851). - Indien, Enispe c., Fruhstorfer: A. Seitz, Groß- ? Südost schmett. Erde, v. 9, p. 445 (1911). Tibet,

Perak

- subsp. cy cnus Westw. E. c., (E. Doubleday \&) Westwood, ut antea, p. 330 (nota) (1851). - Forsfield \& E. Moore, Cat. Lep. Ins. Mus. E.-Ind. Comp., v. 1, p. 212 (1857). - Herrich-Schüffer, Corr.-Bl. Zool.-Min. Ver. Regensb., v. 19 , p. 88 (186.5). - W. F. Kirby, Cat. Diurn. Lop., p. 117 (1871). - Marshall \& Nicéville, Butt. Ind. Burm. Ceyl., v. 1. II, p. 303 (part.) (1883). - O. Staudinger (\& Schatz), Exot. Schmett., v. 1, p. 191 (1887). - Elwes (\& O. Möller), Trans. Int. Soc. London, p. 281, 333 (1885). - Manders, 1. c., p. 520 (1890). - Elwes, Proc. Zool. Soc. London, p. 271 (patria: Perak!) (1891). - Swinhoo, Trans. Ent. Soc. London, p. 276 (1893). - Nicéville, Gazettecr Sikhim, List Butterfl., p. 130 (part.) (1894). - F. Moore, Lep. Ind., v. 2, p. 201 (part.), t. 157, f. 1,1 a $\left(O^{*}\right)$ (? typicus), f. 1 b (Q) (1895). - Bingham, Faun. Brit. Ind., Butterff., v. 1, p. 199 (part.) (1905). - H. Stichel, Gen. Ins., fasc. 31 , p. 13 , (part.), t., fig. 7 (Q) (1905). - E. c. c., Fruhstorfer: A. Seitz, Grofschmett: Erde, v. 9, p. 448 (non t. $105 \mathrm{c}:=E$. c. verbanus o) (1911). - E. c., Tytler, Journ. Bombay Nat. Hist. Soc., v. 21, p. 55 (1911). - W. H. Evans, 1. c., v. 21, p. 572 (part.) (1912). - ? Id., 1. c., v. 23, p. 538 (patria: S.O.-Tibet!) (1915). - Id., l. c., v. $29,[1,297$, t. 16 , f. E 11. 2 (1923). - Antram, Butterfi. Ind., p. 122, 1. 251 (1924). - N. D. Riley \& Gabriel, Cat. Type Specim. Rhopal. Brit, Mus., pars 1, p. 16 (1924). - W. II. Evans, Identif. Ind. Butterfl., p. 87, t. 16, f. E 11. 2 (1927).

erosa Stich. S. c. forma e., H. Stichel, Gen. Ins, fase. 31, p. 13 (nota) (1905).

subsp. verbanus Fruhst. E. c. v., Fruhstor- Bhutan fer: A. Seitz, Großschmett. Erde, v. 9, p. 448 , t. 106 a $\left(0^{\prime}\right)$, t. $105 \mathrm{c}$ (Q: E. cycnus, corr., p. 1135) (1911 \& 27). - W. H. Evans,

\section{Sikkim,}

Bengalen,

Assam,

Burma,

? Südost-

Tibet,

Perak 
Journ. Bombay Nat. Hist. Soc., v. 29, p. 797 (1923). - id., Identif. Ind. Butterfl., p. 87 (1927).

E. cycnus (part.), ut antea: Marshall \& Nicéville (1883); Nicéville (1894); Bingham (1905); Stichel (1905); Evans (1912); Antram 3 (1926).

\section{Addenda et Corrigenda.}

Pag. 4, lin. 18 (Amathusiidae) adde: ,Sonan, Trans. Nat. Hist. Soc. Formosa, v. 20, p. 34 (1930).

Pag. 7, lin. $15 \mathrm{ab}$ imo adde: Morphinae, ,S. H. Seudder, Butterfl. East. Un. St. Canada, v. 2, p. 1048, 1049 (1889)."

Pag. 13, lin. $1 \mathrm{ab}$ imo (Amathusia phidippus) adde: „Tutt, Nat. Hist. Brit. Lop., ₹. 1, p. 77 (1899)."

Pag. 29, lin. 17 (Tharmantis) adde: „S. H. Scudder, Butterfl. East. Un. St. Canada, v. 2, p. 1048 (1889).“

Pag. 48, lin. 23 lege: "Thaumantis" pro ,T."

Pag. 51, Genus Stichophthalma lin. 10 adde: „S. H. Scudder, Butterfl. East. Un. St. Canada, v. 2, p. 1049 (1889)."

Pag. 58, subsp. formosana, adde: ,Stichophthalma how$q u a$ (non Thaumantis $h$. Westw.), Matsumura, Nihon Senchu Drukai (Thousand Ins. Japan), v. 4, p. 146, t. 78, f. 2 (1907)."

Pag. 84, lin. $16 \mathrm{ab}$ imo adde: „Orcas Dubia, W. F. Kirby: Hübner \& Geyer, Samml. Exot. Schmett., Neue Ausg., t. 84 (1894 $-95)^{4}$

Pag. 118, lin. 26 pro ,lit toralis" (nom. praeoce. Rothsch., 1905) instit. est: "litore $a^{\prime \prime}$.

Pag. 154, lin. 20 adde: „D. tullina (!) hainanensis, Sonan, Trans. Nat. Hist. Soc. Formosa, v. 20, p. 34 (1930)." 


\section{Index.}

Synonyme und ungültige Namen sind kursiv gedruckt. - Namen, die sich auf derselben Seite wiederholen, sind nur einmal aufgeführt.

\section{Familia, Subfamiliae, Tribus, Genera, Sectiones, Cohorfes.}

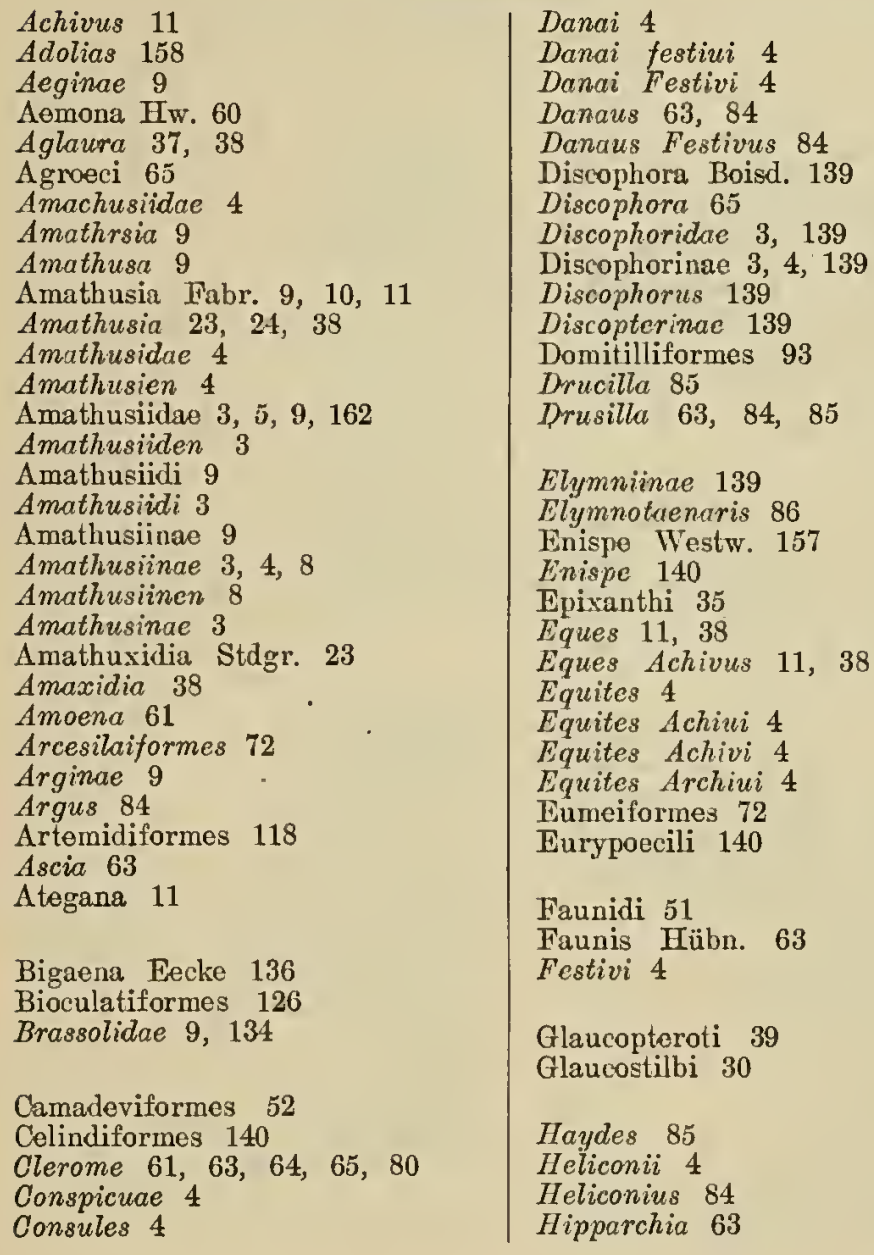


Hipparchiae 5

IIipparchidae 5

Horsfioldiiformes 86

IIyades 85

IIyanthis 134

IIyantidae 134

IIyantiden 134

Hyantiidae 134

Hyantinae 3, 134

Hyantis Hew. 134

IIyautis 134

\section{Iaera 80}

Kringana 29, 30

\section{Melanocyma 65}

Melainarini 132

Mitocerus 11

Moera 11, 38, 140

Moerae 4, 139

Morfinos 8

Morphidac 5, 6, 7, 8, 9

Morphiden 6, 7, 8

Morphiden d. alten Welt 7 , 8

Morphides 6, 7

Morphinac 6

Morphina $6,7,8$

Morphinae 5, 6, 7, 8, 9, 162

Morphindra 48

Morphinen 6, 8

Morphitae 5

Morphites 6

Morpho 6, 11, 29, 38, 63, 84, 140

Morphonidae 8, 9

Morphopsis Oberth. 137

Morphotaenaris 86

Morphotenaris 85, 132

Myopiformes 108

Nandogea 29,30

Nechoformes 146

Nymphales 4

Nymphalcs Gemmati 4

Nymphalidae 5, 6, 134

Nymphaliden 6

Nymphalides 6

Nymphalina 5, 6

Nymphalinae $5,6,7$

Nymphalis 11, 38, 84, 140

Nymphalis Gemmatus 38, 140

Nymphalitae 5

Oginiformes 148

Oreades 4

Oreades Distinctae 4

Oreades Dubiae 4

Oreas 84

Oreas dubia 84

Oreas Dubia 162

Papilionidae 5

Papilionides 5

Pavonia 11

Perakaniformes 20

Perlata 5

Phaoniformes 65

Phidippiformes 11

Poliodermi 45

Potamides 4

Potamides Superbae 4

Potamis 140

Praetores 4

Pseudamathusia 11

Pterourus 11

Satyre 11, 63

Satyridae 5

Satyriden 5

Satyromorpha 5

Satyromorphes 5

Satyromorphi 5

Satyrus 11, 63

Sondaiciformes 151

Selenidiformes 97

Simpliciformes 157

Stenoposcili 151

Stichophtalma 51

Stichophthalma Feld. 51, 162

Stichophthalmia 51

Stichopthalma 51

Sticopthalma 51

Stictophthalmia 51

Stictopthalma 51

Taenarcs 4,82

Taenaridi 82

Taenaridi 51

Taenaris Hübn. 82

Thaumantes 29

Thaumantias 29, 48

Taumantis 48

Tenaris 82, 83, 84

Thaumantis Hübn. 28, 29, 162

Thaumantis 48, 63, 140

Thaumantidae 9

Thaumaturgi 78

Thauria Moore 48

Thauris 48

Thoraessa 9, 23

Thysanuriform Larvae 5

Thysanuriform-Stirps 5

Uraniiformes 88 
Xanthorini 86

Xeuxidia 38

Xanthotaenia Westw. 80

Zerynthia 140

Zeunidia 38
Zeuxaltis 38

Zeuxamathusia 24

Zeuxidea 37

Zeuxidia Hübn. 37

Zeuzidia 37

\section{Species, Subspecies, Formae.}

aberrans Staud. 93

admiralitatis Rothsch. 99

abdon Fruhst. 96

adamsi Lathy 133

adriana Fruhst. 106

adustata Fruhst. 15

adustatus 15

aerope Leech 78

aesculapus Staud. 112

affinis Kirby 125

affinis 125

agapetha Fruhst. 113, 114

agapethus 113

agasta Stich. 113

agina 148

agrippa Fruhst. 94

agrippina 94

aigion Fruhst. 138

albertisi Oberth. 137

albertisi 138

albicans Hag. 123

albiplaga Rothsch. 135

albocostalis Fruhst. 34

aliris Westw. 48, 49

aliris 50

amathusia Hew. 60, 61

amathusia 62

ambigua Stich. 115

ameinokleir Fruhst. 70

amethystina (Discophora)

Stich. 157

amethystina (Zeuxidia) Stich. 40

amethystus IButl. 37, 39

amethystus 40

amitaba Fruhst. 112, 118

amplifascia Rothsch. 51

amythaon Doubl. 24, 25

amythaon 26

anableps Snell.-Voll. 98

anableps 98, 99, 100

anaxilla Fruhst. 43

andamanensis Fruhst. 16

andamanensis 145

andamanica 16

andamensis Stauding. 145

angustifascia Joic. \& Talb. 138

anna Fruhst. 129

annella Stich. 108

ansuna Fruhst. 111 antonia Röb. 55

anulata Stich. 136

appina Fruhst. 107

arcesilas Stich. 73

arcelilaus Fabr. 64, 72, 73, 74, 75

archbaldi Tytl. 57

aneia Fruhst. 129

arfakia Strand 126

aristides 142

aroana Fruhst. 114

arrenopia Fruhst. 17

artemides Fruhst. 123

artemis Snell.-Voll. 119, 120

artemis $100,120,121,122,124$

artemis subsp. nov. 126

assama 77, 78

assamus Westw. 77, 78

astrolabensis Stich. 137

astrolabiensis 137

atesta Reb. 99

attina Fruhst. 103

aurelia Oram. 45, 46, 47

aureliana Honr. 47

aureliana 47

aurelius $45,46,47$

auriflua Fruhst. 97

automola Kirsch 119

automolus 119

avarca Fruhst. 131

baliensis Rothsch. 73

bambusae Feld. 149

banibusae 149, 150

bangkaiensis Fruhst. 150

bankensis Rothsch. 73

barbata Kirby 125

barbata 123, 125

barrauti Moult. 71

batuensis Rothsch. 82

baweanica Fruhst. 14

besa Hew. 71

besa 73

beza 71

biakensis Joic. \& Talb. 138

biakensis 138

binghami Fruhst. 18, 19

bioculata Guér.-Mén. 130, 131

bioculata 130, 131 
bioculatus $85,130,131$

birchi Dist. 87

birchii 87

bisae Rothsch. 93

blandina Fruhst. 120, 121

boisduvalii 44

borneensis (Amathusia) Fruhst. 19,20

borneensis (Faunis) Fruhst. 72

bougainvilleana Strand 99

bowringi Joic. \& Talb. 58

brunnifascia Joic. N. \& Talb. 138

burra Stich. 82

buruensis 101

busiris Westw. 80, 81

busiris 81,82

butleri Oberth. 93

camadeva Westw. 52, 53

camadeva 53, 54

camadevoides Nicév. 54

camadevoides 53

camboria Hew. 54

camens 74

camaronensis Rothsch. 132

candica 33

candida Fruhst. 109

candika Fruhst. 32, 33

canens Hübn, 72

caneus 72

carfinia Fruhst. 67

cassiae 89

catanen Fruhst. 104

catanea 104

catops Westw. 102

catops 98, 100, 101, 104, 105,

$106,107,116,120$

catops subsp. nov. 107

celebensis Fruhst. 17

celebensis Holland 149

celinde Stoll 140, 141

celinde $143,144,145,147,151$, 155

celinde var. 143

celsa Fruhst. 121

ceylonica Fruhst. 150, 151

charon Stauding. 131

charondas Fruhst. 131

charonides Stauding. 131

chatra Fruhst. 37

cheops Feld. 146

cheops 147, 148, 156

chersias Fruhst. 15

chersonesia Fruhst. 42, 43

chionides Godm. \& Salv. 114

chitone Hew. 69

chitone 68 chthonia Fruhst. 23

chuni Joic. \& Talb. 60

coerulilata Eecke 26

colarima Eecke 104

concolor Hulst. 119

continentalis Stauding. 141, 143

convergens Rothsch. 117

coriotincta Stich. 17

cyclopides Fruhst. 115

cyclops Röb. 34

cyclops Stauding. 113

cycnus Westw. 161

cycnus 162

cyme Fruhst. 72

d'albertisi 137

dampierensis Rothsch. 106

danalis Frubst. 126

decempunctatus $\mathbf{7 7}$

delunata Fruhst. 103

deo Nicév. 145

depupillata Fruhst. 35

derhion Fruhst. 138

desdemona Stauding. 128

desdemona 129

despoliata Stich. 152

diadema Fruhst. 92

diana Butl. 92

digulica Hulst. 104

dilucida Honr. 25, 26

dilucida 26

diluta Fruhst. 17

dilutus 17

dimona Hew. 127

dimona 128, 129, 130

dimona var. (ab.) 108

dimonata Stich. 128

dina Stauding. 130

dinora Gr. Smith \& Kirb. 129

dinora 130

diops Snell.-Voll. 94

dioptrica Snell.-Voll. 111

dioptrica 100, 112, 113

diores Doubl. 30, 31

diores 31

dis Nicév, 147

dis 146

dohertyi 100

dohrni Fruhst. 42, 44

domitella 94

domitilla Hew. 93, 94

domitilla 94

doubledaii Westw. 41, 44

doubledaii 42,43

doubledayi $41,42,43$

druentia Fruhst. 123

duplex Stich. 92

durania 160

duranius Fruhst. 160 
editha Ril. \& Godfr. 54

eichhorni 118

electra Fruhst. 124

eleusina Fruhst. 120

emarginata Fruhst. 135

enervata Stich. 161

engamon Fruhst. 147

enomia Fruhst. 95

enthymius 159

eos Hell. 123

epidesma Fruhst. 17

erasimus Fruhst. 148

erosa Stich. 161

errhephoria Frulsst. 116, 117

errhiphoria Frubst. 117

eugenia Fruhst. 115

eumea 76

eumeus Drury 75, 76

eumeus 77

euthycrite Frulist. 47

euthymius Doubl. 158

euthymius 160

eutropius Fruhst. 14

eutymius 160

evansi Tytl. 57

excelsa Rothsch. 45

excelsa 78

excelsus Fruhst. 78

farona Fruhst. 99

faunala 76

faunula Westw. 65, 79

faunuloides Nicév. 79

ferdinandi. Fruhst. 114

fergussonia Fruhst. 116

fimbriats Kirb. 106

fimbriata 106

formosana Fruhst. 58, 162

tredericki 16

friderici Fruhst. 16

triderici 16

fruhstorferi (Faunis) Röb. 69

fruhstorferi (Stichophth.) Röb. 55

fruhstorfori Stich. 145

truhstorferi 57

fuligino:a Gr. Smitb 135

fulvida Butl. 102, 103

fusca 58

gabriela Fruhst. 20

galaecia Fruhst. 105

gemmata Fruhst. 93, 119, 124

gemmata Stich. 110

gigas Staud. 101

gisela Fruhst. 121

godfreyi Rothsch. 54

gorgo Kirsch 126

gorgo 127

gorgophone Fruhst. 127

gracilis Butl. 67, 68

gripus 76

grisescens Rothsch. 131

hadina Frubst. 109

hageni Röb. 136

hainana Crowl. 31

hainanensis Fruhst. 154, 162

haynei Tytl. 63

helvidius Fruhst. 147

helvola Stich. 136

hodeva Hew. 134

hodeva 136

hollandi Fruhst. 92

bonrathi Staud. 86,96

honrathi 42, 97

horsfeildii 86

horsfieldi 42, 86, 87

horsfioldii Feld. 42

horsfieldii Swains. 86, 87

horsfieldii 42, 45, 88, 96

howqua Westw. 51, 57

howqua 56, 57, 58, 59, 162

luulstaerti Stich. 104

humboldti Fruhst. 122

hyperbola Kirsch 118,119

hyperbolus 113, 118, 119

iaira 91

iairus 90

ida Honr. 95

idae 95

ikonion Fruhst. 66

incerta 77

iucertus Staud. 77

incisa Fruhst. 21

indica Stauding. 153, 155

indica 156

indra 131

insularis Dohert. 26

infumata Stauding. 135

infumata 123

insularis Rothsch. 130

interfaunus Rothsch. 117

intermedia Crowl. 50

intermedia Rothscb. 160

intermedia 50

intermodians Hulst. 120

intermedius Röb. 68

interrupta Strand 105

jaira $89,90,91$

jairus Cram. 90, 91

jairus Fabr. 84

jairus 89,90 
jamesi But.l. 124

jamesi 126

jathrippa Fruhst. 103

javaensis 12

jobina Fruhst. 103

kajuna Fruhst. 104

kapaura Fruhst. 129

kenricki Beth.-Bak. 133

kirata Nicér. 75

kirschi Stauding. 117

kirschi 116, 117

kleis Semp. 70

klugius Zink. 31, 32

klugius 33,34

kubaryi Stauding. 114

kubaryi 115

kühni Röb. 18

kumusii Rothsch. 137

lacrimans Fruhst. 110, 119, 123

laretta Fruhst. 103

laretta 104

lathyi Fruhst. 50

lautensis Rothsch. 71

le Joic. \& Talb. 59

lena Atkins 62

leonteus 72

leontius 74

lepida Moore 150

lepida 151

lepidea 150

leto Truhst. 93

leucida Semp. 67

leucis Feld. 67

leva 60,62

licinia Fiulıst. 112

litorea Stich. 162

littoralis (Taen. mailua) Rothsch. 118,162

littoralis (Taen. schönbergi) Rothsch. 133

limbata Fruhst. 123

lombokiana Fruhst. 14

louisa Wood.-Mas. 55

louisa 56

lucida Fruhst. 26

lucipor Westw. 32

lucipor 31, 32, 33

luna Stich. 106

lunata Leech 160

lunatus 160

Iurida 66

luridus Field. 66

luxeri $38,41,44$

luxerii Hübn. 37, 43, 44

luxerii 41,44

luxerrei 44 macrophthalma Fruhst. 95

macrophthalmus 95

macropina Fruhst. 100

macrops Feld. 100

macrops 100, 101

mailua $\mathrm{Gr}$. Smith 118

majada Fruhst. 14

mambarensis Rothsch. 137

maneta Hulst. 111

masoni Fruhst. 39

marinus 89,91

masina Fruhst. 20, 22, 23

mathilda Jan. 56

meeki (Morphopsis) Rothsch.

\& Jord. 138

meeki (Taenaris) Rothsch. 126

molaena Tytl. 160

melanomata Stauding. 135, 136

melanops Wecke 15

melanops Gr. Smith 125

melinda Feld. 149

menado Hew. 68

menado 69

menetho 142, 144, 145, 148

mera Fruhst. 126

merana Fruhst. 110

microphthalma Hell. 135, 136

microps Gr. Smith 127

microps Stauding. 66

milnei Rothsch. 137

milous 160

milvus Stauding. 160

mindorana Fruhst. 148

mindorana 148

miscus Fruhst. 116

misolensis Rothsch. 116

miyana Fruhst. 58

moiarum Fruhst. 77

molaus 96

monops Fruhst. 119, 123

montana Stich. 96

morosa Stich. 87

murinus 89

muscina Stich. 156

myalechoides 106

mylaecha Westw. 107

mylaecha 116

mylaechoides Fruhst. 106

myloechoides 106

myopina Fruhst. 121

myops Feld. 115

myops $116,117,118,122$

nagaensis Rothsch. 54

natuna Fruhst. 22

necho Feld. 146

necho 144, 147

nephus 44

neumogeni Leech 59 : 
neumogeni 59

niasana (Amathusia) Fruhst. 15 niasana 73

niasanus (Faunis) Fruhst. 73

nicasius Fruhst. 107

nigricans Hag. 122

nigricans 122

nigrigans 122

nicevillei Fruhst. 43

nicévillei Röb. 53, 54

nicévillei 42

nivescens Rothsch. 133

noureddiu 36

noureddin Westw. 36

noureddin 36,37

nourmahal Westw. 59

nourmanhal 59

nox Kirb. 91

nurinissa Nicerv. 60

nysa 89

oberthüri Stich. 61

obscura Btl. 81

occidentalis Rothsch. 117

occulta Gr. Smith 87, 88

ochraceofusca Honr. 19

ochraceofusca 19

ochreo/usca 19,20,23

octacilia Fruhst. 27

oda 34

odana God. 32, 33, 34

odana 34,35

odora Fruhst. 147

odorata 147, 148

offaka Fruhst. 129

ogina God. 148

oginn 146, 149

onesimides Fruhst. 113

onesimus Butl. 113

onolaus Kirsoh 94,95

onolaus 95, 96

opulenta Fruhst. 87, 119

opulenta Stich. 124

orasis Fruhst. 21

orhicularis Stich. 147

orientalis Rothsch. 113

ottomana Butl. 27

oxyophthalma Stich. 135

palawana Fruhst. 18

palawanicus 147

palawanus 18

pallida Fruhst. 131

pallida Rothsch. 135

pallidior Hag. 73

pamphaga Kirsch 103, 104

pamphaga 103, 105

pamphagus 102, 103, 104 pandemos Fruhst. 92

panwila Fruhst. 35

paramita Fruhst. 35

parallelus Rothsch. 111

patalena Westw. 20

peali 62

pealii Wood-Mas. 61, 62

pelagia Fiuhst. 108, 109

perakana Honr. 20, 21

perakana $16,19,22$

perakensis Stich. 144

perinthas Fruhst. 27, 28

perplexus Rothsch. 108

phaon Erichs. 65

phaon 68

phidippe 12

phidippus Johans. 11, 12, 13, 162

phidippus 14, 15, 16, 17, 18, 21, 142

phidippus ab., var. 16, 20

phiduppus 20

philippina (Amathuxidia) Moore 27

philippina (Discophora) Moore 148

phippsi Joic. \& Talh. 138

phorcas Westw. 97, 98

phorcas 99, 100

pishuna Fruhst. 35

pishuena 35

plateni (Amathux.) Staud. 28

plateni (Faunis) Staud. 71

plateni (Taenaris) Staud. 88

pleiops 93

plconasma Röh. 68, 69

pollicaris Butl. 18

polychroma Hag. 82

porthaon 27, 28

portheos 15

portheus 25

praexedes 116

praxedes Fruhst. 116

praxedes 116

prodiga (Taenar. agrippa)

Fruhst. 94

prodiga ('Taenar, wahnesi)

Fruhst. 110

prodigiosa Fruhst. 39, 44

propinqua Stich. 147

pryeri Butl. 41

pseudaliris Butl. 48, 49

pseudomacrops Rothsch. 102

pumilio Eocke 136

pylaon Feld. 26

quadriocellata Strand 110

quadriocellata 110

queenslandica Rothsch. 126 
rafaela Fruhst. 111

ramdeo Noore 31

rebeli Fruhst. 97

reducta Rothsch. 110

retracta Fruhst. 15

retrogada Fruhst. 14

ribbci 22

rileyi Hulst. 113

ritsemae Fruhst. 97

rosseli Fruhst. 118

rosseliana Rothsch. 107

rothschildi Fruhst. 119

rothschildi Gr. Smith 108

ruhstorferi 145

sadija Fruhst. 81

samadhi Fruhst. 73

sappho Semp. 69, 70

saturatior Fruhst. 95

schonbergii 19

schönbergi Fruhst. 132

schönbergi Honr. 19

schönbergi 18, 19, 133

scylla Staud. 99

sekarensis Fruhst. 96

seliarensis 97

selene Westw. 101

selene 98, 100, 101, 102, 104

105,116

selene var. 100

selenides Staud. 103

seminechis 143

seminecho Stich. 143, 144

semperi Feld. 45

semperi Moore 157

senaria Stich. 111

setakwacnsis Rothsch. 137

siamensis (Stichophth.) Rothsch. 56

siamensis (Thauria) Rothsch. 50

sibulana Honr. 45

sidus Fruhst. 110, 119, 124, 127

sidus Stich. 97

sigirya Fruhst. 36

significans Stich. 151

silhetensis 159,160

simonetta Fruhst. 123

simplex Frulist. 127

simplex Stauding. 157

sondaica Boisd. 151, 152

sondaica 153,155

sondaica ab. 154

sophaineta Fruhst. 130

sordidior Rothsch. 130

sorronga Fruhst. 128

sparta Nicév. 56

spiloptera Nicév. \& Möll. 156

staudingeri Honr. 122 staudingeri Röb. 21, 22

staudingeri 120

sticheli Fruhst. 124

stomphae 70

stomphax Westw. 70, 71

subluna Rothsch. 106

submylaecha Rothsch. 106

subquadriocellata Strand 110

succulenta Stich. 44

suffusa Leech 57,58

suffusa 58, 59

sulanus Fruhst, 69

sultanus Sticl. 36

suluana 69

sumatrana Fruhst. 42

sumatranus Rothsch. 67

suprema Fruhst. 28

sylhetensis 160

syllus Fruhst. 69

symphronia Fruhst. 152

taenia Fruhst. 21

tainia Fruhst. 108

tainides Frubst. 109

tenuitata Eecke 73

ternatana Fruhst. 101

tesselata Moore 158, 159

tesselata 160

tesselatus 159

tetrica Stich. 102

thaema Fruhst. 130

therionarca Frubst. 45

thessalus 24

thoanthea Firuhst. 22

timesides Fruhst. 121

timora Westw. 142, 143

timora 142,144

tineutus Fruhst. 125

tonkiniana Frubst. 58

tonkinensis Fruhst. 61

timesias Kirsch 112

timesias, Albino 112

trioculatus 110

trita Stich. 129

tullia. 151, 152, 153, 154, 155 , 156

tulliana Stich. 153

tullina 162

tullius 153

turaica Hulst. 104

turdula Fruhst. 107

tytleri Rothsch. 57

ula Rothsch. \& Jord. 138

umbonia Fruhst. 98

umbonia 98

umbonius 98

undata Stich. 142

unipupillata Fruhst. 105 
urania L. $82,83,88,89$

urania $87,91,92,124$

uranus Staud. 98

uranus 99

vaneeckei Hulst. 105

vanhaasterti Hulst. 110

vanhaasterti 104

varda Fruhst. 142

verbanus Fruhst. 161

verbeeki Fruhst. 117

versteegi Eecke 119

victrix Staud. 41

virgata Btl. 22

virgata 17

virgatus 17

vulcania Stich. 106

vulcanica 106

wahnesi Hell. 109

wahnesi 108

wahnesi-trioculatus 110 wallacei Feld. 40

wattina Fruhst. 112

wandammensis Joic. \& Talb. 133

wedana Fruhst. 34

westwoodi Staud. 105

westwoodi 25

weylandensis Joic. \& 'Talb. 133

wilhelma Röb. 59

wollastoni Rothsch. 133

xanthophthalma Röb. 136

yantiva Fruhst. 35

zaitha Frubst. 129

zal Westw. 154

zal 142, 152, 157

zenada Fruhst. 120

zenica Fruhst. 68

ziada Fruhst. 121

zibulana 45 



\section{W. J UNK, Verlag und Antiquariat für Entomologie Berlin W. 15}

Pars 35, 37, 39: F. Bryk, Papilionidae. 1929-1930. 675 p.

36: H. Neustetter, Helieoniidae. 1929. 136 p.

38, 40, 41, 44: H. Stichel, Riodinidae 1930-1931. 795 p.

42: F. Bryk, Dioptidae. 1930. 65 p.

43, 46, 4S: H. Gaede, Satyridae. 1931. 759 p.

47: H. H. Shepard, Hesperidae: Subfam. Pyrginae I. 1931. 144 p.

49: M. Gacle, Drepanidae. 1931. 60 p.

50: II. Gaede, Mimallonidae. 1931. 21 p.

51: H. Stichel, Brassolidae. 1932. 115 p.

52: M. Gaede, Aganaidae. 1932. 39 p.

53: G. Talbot, Pieridae I. 1932. 320 p.

55: H. Sehiissler, Saturniidae I: Subfam. Attacinae. 1932. 81 p.

Im Druek:

56: H. Schiissler, Saturniidae II.

In Vorbereitung:

F. Bryk, Lymantriidac, Zygaenidae (excl. Zygaena speeies palaearet.).

A. Busck, Stenomidae.

A. Corti, Agrotinae palaearetieae.

J. Draeseke, Lyeaenidae.

M. Gaede, Notodontidae, "Tineoidea":
1. Ineurvarïdae
5. Opostegidae
9. Momphidae
2. Tiseheriidae
6. Tineidae
10. Elaehistidae
3. Heliozelidae
7. Gelechiidae
4. Neptieulidae
8. Coleophoridae
11. Cosmopterygidae
12. Lyonetiidac
13. Cemiostomidae.

J. Kremky, Danaididae, Pyralididae.

L. B. Prout, Sterrhinae.

H. Schüssler, Saturniidae III., Eupterotidae.

H. H. Shepard, Hesperiidne II et III.

H. Stichel, Diseophoridae, Amathusiidae, Hyantidae, Nymphalinae, Apaturinae.

E. Strand, Noetuidae.

G. Talbot, Pieridae II. 
Die Gesellschaft für Höhlenforschung und $\mathrm{Höh}$ lenkunde $\mathrm{zu}$ Berlin beabsichtigt herauszugeben (das Manuskrip乞 steht vor dem Abschluß):

\section{Caralogus Animalium Cavernarum}

\section{Cafalog der Höhlenfauna}

\section{Caralogue of the fauna of the caves}

\section{Cafalogue de la faune cavernicole}

Dicse Arbeit enthält die Aufstellung der gesamten lebenden Tierwelt in Höhlen, deren Größe den Menschen den Zutritt ermöglicht. - Die Fauna der Micro-Cavernen (wie Maulwurfsnester etc.) und die der Erdspalten, sowie die Quellenfauna sind also nicht mitberücksichtigt. - Das Werk bchandelt nicht nur die Tierwelt, dic nur in Höhlen vorkommt und sich dem Höhlenlcben besonders angepaßt hat, sondern die Gesamtheit derjenigen in Höhlen vorkommenden Tiere, von denen naehgewiesen ist, daß sie in einer irgendwie geartcten biologischen Beziehung zu den Höhlen stehen. Ausgeschlossen sind nur solche Tierc, von denen ganz offenbar ist, daß cs an solcher biologischen Beziehung felılt.

Die Einteilung des Werkes ist so übersichtlich getroffen, daß der Benutzer des Werkes stets sofort die von ihm gewünsehte Literatur über die Höhlen und deren Fauna findet:

1. Die gesamte Höhlenfauna-Literatur (in Werken und Zeitschriften).

2. Hinweis auf die Herkunft der Höhlentierwelt, ihre Lebensbedingungen und ihre Anpassung an das Höhlenleben.

3. Die Höhlen geographisch geordnet (also naeh Erditeilen, Ländern etc.) sowcit ein Vorkommen lebender Tiere in der Literatur bckannt ist. - Angabe der auf jede einzelne Höhle sieh beziehenden Literatur in faunistisch-geordneter Übersieht.

4. Die Tierwelt systematisch und geographiseh nach Fundorten (Höhlen) geordnet.

5. Register der Tiernamen (Synonyme besonders gekennzeichnet).

Das Werk, welches nur bei genügender Teilnahme von Interessenten erscheinen kann, erhalten Mitglieder der Gesellsehaft mit eincm Rabatt von $25 \%$. Zunächst unverbindliehe Bestellungen sind an die Gesehätsstelle der Gesellsehaft für Höhlenforschung und Höhlenkunde, Berlin SW. 11, Kleinbeerenstr. 3 , oder an den Verlag W. Junk, Berlin W. 15, zu leiten. Diesen Interessenten wird dann nach Eingang einer genügcnden Anzahl von Anmeldungen ein ausführlicher Prospekt mit Probe-Seitc und Preisangabe zugesandt. Das Manuskript ist soweit fertiggestcllt, daß im Herbst dieses $J$ ahres mit dem Druck des Werkes begonnen werden kann, wenn die finanzielle Grundlage des Unternelumens dureh eine genügende Anzahl von Subskribenten geschaffen ist.

Druck von Gustav Feller, Neubrandenburg. 